\title{
Solid-Polymer-Electrolyte Fuel Cells
}

\author{
Thomas F. Fuller \\ Ph.D. Thesis \\ Department of Chemical Engineering \\ University of California \\ and \\ Materials Sciences Diwision \\ Lawrence Berkeley Laboratory \\ University of California \\ Berkeley, CA 94720
}

July 1992

This work was supported by the Assistant Secretary for Conservation and Renewable Energy, Office of Transportation Technologies, Electric and Hybrid Propulsion Division, of the U.S. Department of Energy' under Contract No. DE-AC03.76SF00098. 


\title{
Sol1d-polymex-electrolyte Fuel Cells
}

by

\author{
Thomas F. Fuller
}

\begin{abstract}
A general transport model for polymer electrolytes is presented. The model is based on concentrated solution theory and irreversible thermodynamics. The appropriate thermodynamic driving forces are developed. The transport properties are identified and suitable experiments devised.

The transport number of water in Nafion 117 membrane over a wide range of water contents is determined experimentally using a concentration cell. The transport number of water, the ratio $t_{0}^{m} / z_{0}$. is about 1.4 for a membrane equilibrated with saturated water vapor at $25^{\circ} \mathrm{C}$, decreases slowly as the membrane is dehydrated, and falls sharply toward zero as the concentration of water approaches zero. The relationship between the transference number, the transport number, and the electroosmotic drag coefficient is presented, and their relevance to water-management in soldd-polymer-electrolyte fuel cells is discussed. Results are compared with other data avallable in the literature and with the theoretical maximum.

A mathematical model of transport in a solid-polymer-electrolyte fuel cell is presented. A two-dimensional membrane-electrode assembly is considered. Water management, thermal management, and utilization of fuel are examined in detail. The membrane separators of
\end{abstract}


these fuel cells require sorbed water to maintain conductivity; therefore it is necessary to manage the water content in membranes to ensize efficient operation. Because the equilibrium sorption of water between the gas phase and the polymer-electrolyte depends strongly on temperature, water and thermal management are interrelaced. The rate of heat removal is shown to be a critical parameter in the operation of these fuel cells. Current-voltage curves are prasented for operation on air and reformed methanol.

The equations for convective diffusion to a rotating disk are solved numerically for the case where a consolute point is found between the concentration in the bulk and that at the surface. A singular-perturbation expansion is pxesented for the condition where the bulk concentration is nearly equal to the consolute-point composition. Results are compared to Levich's solution for constant pro. perties and with his analysis of an experimental systern. 
Abstract

Table of contents

Acknowledgements

whapter 1. Introduction

1.1 Conducting polymers and ionomeric membranes

1

1.2 Solld-polyner-electrolyte fuel cells

1. 3 objectives and approach

Chapter 2. Theory of Transport in Polymer Electrolytes

2.1 Introduction

2.2. Thermodynamic driving forces

14

2.3 Transport properties

15

2.4 Concentration scale

2.5 Bulk hydrodynamic flow

2.6 Equilibrium and hysteresis

18

21

2.4

25

Chapter 3. Mode1Ing

3.1 Introduction

$\begin{array}{ll}3.1 & \text { Introduction }\end{array}$

3.3 Electrolyte phase 35

3.4 Chemical reactions 38

3.5 Boundary conditions 40

3.6 integration

40

3.7 Energy balance

3.8 Numerical Approach

Chapter 4. Heasurement of the Transport Number of Water

4.1. Introduction

4.2 Theory

4.3 Experimental procedure

4.3 Results and discussion

Appendix A Computer program 
Chapter 5. Diffusion Coefflcient of Wator

5.1 Introduction 80

5.2 Measurement of the Diffusion Soefficient 82

5.3 Conductometric method 88

5.4 Mutual and Intra Diffusion Coefficients 92

5.5 Calculation of Binary Diffusion Coefficients 96

Chapter 6. Steady-state Results and Water Management

6.1 Introduction 108

6.2 Unit cross section 110

6.3 Isotherm case $\quad 114$

6.4 Nonisothermal case $\quad 124$

Appendix B Physicochemical properties 132

Appendix C Computex program 141

Chapter 7. Translent Analyses of Solid-Polyiner-Electrolytes

7.1. Introduction 169

7.2 Ttransient response 170

Appendix D Transient analysis $\quad 176$

Appendix E Computer programs 187

Chapter 8. Convective Diffusion neax a Consolute Point

8.1 Introduction 216

$\begin{array}{ll}8.2 \text { Analysis } & 217\end{array}$

$\begin{array}{ll}8.3 \text { Ferturbation analysis } & 227\end{array}$

8.4 Mass Transfer $\quad 232$

8.4 Discussion $\quad 233$

Appendix E Nonclassical analysis 239

$\begin{array}{ll}\text { Appendix F Computer program } & 247\end{array}$ 
Acknowledgements

Above all I wish to thank Professor John Newman. He sets an exemplary standard of excellence - his students are always a priority. Although this trait may be difficult to measure, it will not be forgotten. I also wish to thank my present and former coworkers in the group.

I would like to acknowledge the members of the fuel-cell group. MEE-11 at Los Alamos National Laboratory for their help and valuable advice, as well as E. I. du Pont de Nemours and Company for providing the mombranes.

This work was supported by the Assistant Secretary for Conservation and Renewable Energy, Office of Transportation Technologies, Electric and Hybrid Propulsion Division of the $U$. S. Department of Energy under Contract No. DE-AC03-76SF00098. 
Chapter. 1

Introduction

\subsection{Conducting Polymers and Ionomeric Membranes}

In recent years there has been extensive development of electronic, ionic, and mixed conducting polymers [1], [2]. Conducting, polymers are a part of several commercial processes, the electrochemical production of chlorine for example. The first widely used perfluorinated ionomeric product was Nafion , introduced in the sixties. Since then other ionically conducting polymers with good physical properties have become available, and mary electrochemical systems have been based on them. Eisenberg and Yeager [3] review the rech. nology of perfluorinated ionomeric polymers. Ionically conducting polymers have sparked interest for a wide range of applications: solid-state batteries [4], drug delivery systems, electrochemical reactors for separation processes, and electrochemical sensors [5], to name a few. One in particular, the solid-polymer-electrolyte fuel cell, has seen renewed interest of late [6].

Ionically conducting solids are employed in several fuel-cell systems, such as the solid-oxide fuel cell, and the solid-polymerelectrolyte fuel cell. One early application of perfluorinated ionomer membranes was the fuel cell for the Gemini space program [7]. Current: work is focused on the development of alternative power sources for vehicles [8] using reformed methanol or direct oxidation of methanol. Appleby and Foulkes [9] provide a comprehensive review 
of the operation of a number of fuel-cell systems, including their applications and the status of their development programs. Solidpolymer-electrolyte systems have the advantage of operating at low temperatures and being completely tolerant to carbon dioxide. Until recently the solid-polymer-electrolyte fuel cell required high platinum loadings because of the low temperature of operation. The cost of the catalyst was acceptable only for specialized applications, such as the space program. Successful operation of fuel cells with low platinum loadings [10] has increased interest in the commercialization of these fuel cells.

\subsection{Solid-polymer-electrolyte fuel cells}

Figure 1 shows a typical solid-polymer-electrolyte fuel cell consisting of a solid-polymer-electrolyte and two gas-diffusion electrodes. These fuel cells are envisioned to run on hydrogen from reformed methanol and air, generating about one volt at open clrcuit. The overall reaction is

$$
\mathrm{H}_{2}+3 / 2 \mathrm{O}_{2} \rightarrow \mathrm{H}_{2} \mathrm{O}+\text { heat }+ \text { electrical work. }
$$

The gas-diffusion electrodes are polymer-bonded porous-carbon structures with platinum catalyst on the carben surface. More details of the operation of gas-diffusion electrodes and their application in membrane fuel cells can be found in [9], [11], [12]. A thin membrane and two gas-diffusion electrodes are pressed together above the glass transition temperature of the polymer to ensure good contact between 


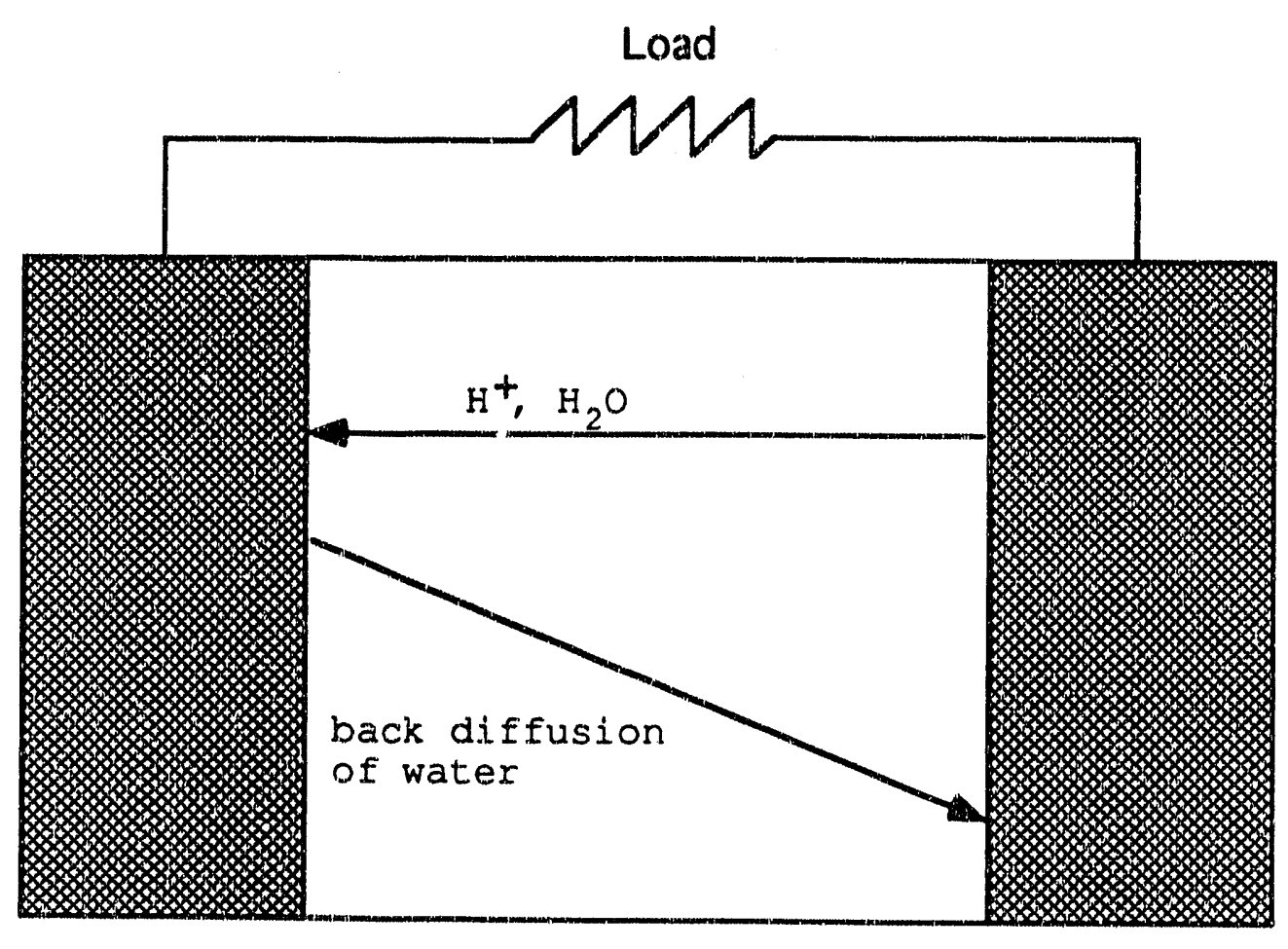

Cathode

Anode

Figure 1. Solid-polymer-electrolyte fuel cell operating on hydrogen and oxygen. 
the polymer, carbon, and platinum. One purpose of the solid polymer electrolyte is to keep the anode and cathode reactants apart. There still must be an ionic path with low electrical resistance between the two.

The hydrogen and oxygen streams are heated and humidified before entering the membrane-electrode assembly. Hydrogen gas diffuses through the porous anode and reacts at the electrocatalyst in a three-phase region containing polymer electrolyte, gaseous reactants, and a carbon matrix which supports the platinum catalyst. Oxygen passes through the other gas-diffusion electrode to be reduced cathodically. At the cathode, the hydrogen ions from the electrolyte react with oxygen at the electrocatalyst sites to form water.

Nafion, a copolymer of tetrafluoroethylene and sulfonyl fluoride vinyl ether, has many ionizable groups. Reference [3] and Pineri and Eisenberg [13] provide detailed information on the structure and properties of Nafion. The general structure is given in figure 2, where $m$ is one and $n$ varies from 5 to 11 , depending on equivalent weight. The incorporation of ionic groups into the polymer has a dramatic effect on j.ts physicochemical properties.

An important aspect of the perfluorinated ionomeric membranes is their ability to take up large amounts of water and other solvents. The increase in water content is accompanied by a reduction in tensile strength and resistance to transport. The water content in the membrane can be as high as 22 water molecules per sulfonic acid group. The first few water molecules are associated with high 


$$
\left.-\left(\mathrm{CF}_{2} \mathrm{CF}_{2}\right)_{\mathrm{n}}-\underset{\mathrm{C}_{2}}{\mathrm{CFO}} \underset{\mathrm{C}}{\mathrm{C}} \mathrm{CF}_{2}-\underset{\mathrm{m}}{\mathrm{CFO}}\right)_{\mathrm{m}} \mathrm{CF}_{2} \mathrm{CF}_{2} \mathrm{SO}_{3}-\mathrm{H}^{+}
$$

Figure 2 . Structure of Nafion. The hydrogen form is shown, $n$ is about 1 , and $m$ varies from 5 to 11 . 
enthalpic changes and are tightly bound. Entropic changes allow more water to be absorbed, but cross linking of the polymer chains prevents total dissolution. Hydrogen ions are the charge carriers in the hydrated membrane and migrate across the membrane by passing from one sulfonic acid group to the next. The sulfonic acid groups are covalently bound to the polymer backbone and do not move.

Perfluorinated ionomeric membranes are not humogeneous on a microscopic scale. There are anorphous, crystalline, and hydrophillic regions. The most generally accepted microscopic model of perfluorosulfonated membranes is the cluster-network model of Gierke and Hsu [14]. They envision the solvent and fixed charge sites separating from the hydrophobic polymer chain. Roughly spherical cells are connected by thin channels. The charge repulsion in these channels prevents coions from passing through the membrane. As the membrane sorbs more water, the cells become larger, and the conductivity increases.

Because perfluorinated fonomeric membranes require sorbed water to maintain sufficient electrical conductivity for practical use, it is vital to manage the water content in the solid polymer electrolyte. Furthermore, since the equilibrium absorption of water from the gas phase into the electrolyte depends strongly on temperature, water management and thermal management are interrelated. Cell efficiency depends on the transport properties of the membrane and may be the critical factor in load-leveling devices, where the kiretics of cel.1 reactions are fast [1]. 
From the inception of the solid-polymer-electrolyte fuel cell, water transport was recognized as a possible problem (15). Water motion is caused by a gradient in fes chemical potential and by the movement of hydrogen ions, which is proportional to the current. For each hydrogen fon that moves from the anode to the cathode, some have estimated that about three or four water molecules are carried along $[16], 117]$.

Hirh curxent densities can result in the transport of water away from the anode-membrane interface at a rate that is greater than the rate at which it can be restored by back diffusion, ox transport from a humidified gas stream. Consequently, high current densities could cause dehydration at the anode-membrane interface. Local dehydration can lead to a degradation in cell performance or cell failure.

The reduction in platinum loading achie $(3)$ depends on the structure of the incerfacial region. The fuel cell can be optimized by designing interfaces with the desired catalytic and mass-transport properties. For the reaction to proceed, four elements are required: hydrogen at the reaction site, a path for the removal of electrons, an ionic path, and a catalyse. Cood contact is made by hot.pressing the membrane and gas-diffusion electrodes together above the glass transition temperature of the polymer. Still, the utilization of the platinum is low, and further improvements are desired.

In tecent review (18), srinivasan points to scale-up, start-up time, and peaking capabilities as the primaxy concerns for developing fuel cells for transportation applications. 


\section{3 objectives and Approach}

Most studies of transport in perfluorinated ionomeric membranes have been associated with the chlor-alkali industry. The environment in the separator of a solid-polymer-electrolyte fuel cell differs markedly from that encountered in the chlor-alkali industry. There is no external electrolytic solution in contact with the separator of a solid-polyner-electrolyte fuel cell. Water content in the membrane is determined by the temperature, the partial steam pressure in the external gas streams at the electrodes, and the current density. Additionally, within the electrolyte of a solid-polymer-electrolyte fuel cell there are only three species present: the polymer with its covalently bound sulfonic acid groups, water, and hydrogen fons. In contrast to other applications, there are no co-fons in the separator. of a hydrogen/oxygen solid-polymer-electrolyte fuel cell.

The distinction of solid-polymer-electrolyte fuel cells, in that they have no external electrolyte, becomes particularly important in modeling and in the development of experimental procedures to determine the transport properties. Water is required for the polymer to maintain conductivity, but is transported to and from the membrane in the gas pirase. If the water vapor is saturated there may also be removal of liquid water. This further complicates the mathematical modeling.

The long-term goals are to improve the spectic power and the transient response of fuel cells. An essential component in the development of the solid-polymer-electrolyte fuel cell is to provide 
a set of theoretical models. These models will be used to analyze experimental data and will improve the design of fuel-cell systems. The objectives here are to establish a general transport model for the conduction process in ionically conducting polymers and to apply this model to the solid-polymer-electrolyte fuel cell.

The model presented is macroscopic, neglecting geometric details. The volume elements we average over are large compared to the microscopic structure. The membrane is treated as a homogeneous continuum. Nevertheless, the transport properties can be interpreted in terms of the microscopic structure and processes in the membrane.

There are numerous membrane-transport mode $-s$, which will be discussed further in chapter three on modeling. The key feature of the model is that it is based on concentrated solution theory. In the membrane of the solid-polymer-electrolyte fuel cell, as well as in many applications of ionically conducting polymers, the concentration of each of the species is of the order of one molar. Coupling between transport phenomena is common. The distinction between solvent and solutes becomes less clear, and interactions among all of the species are important.

The model should be described by parameters that can be obtained by unambiguous physical measurements. Together with the development of a theoretical model, experiments must be carried out to measure the transport properties of the polymer electrolyte. Because of the unique nature of the environment of the solid-polymer-electrolyte fuel cell, specific experiments are needed to mimic these conditions. 
In particular, physical properties will vary with water content, and this dependence must be understood.

It was recognized early on that phenomena in solid materials can be described in an analogous fashion to that which has been developed for liquid electrolytes [19]. The characterization of a wide variety of electrochemical systems with a small number of fundamental physical processes allows for the use of a general model for a variety of diverse systems. Conducting polymers and semiconductors can be described by the same models as those employed for aqueous solutions. This unified approach greatly simplifies the identification of important parameters, verification of models, and analyses of experimental data. Although the mechanisms for conduction of current varies and the applications are diverse, transport phenomena can be described using the concepts of irreversible thermodynamics, which will provide a coherent and unified approach to the characterization of these polymers.

\section{References}

[1] Membranes and Ionic and Electronic Conducting Polymers, proceedings Volume 83-3, edited by E. B. Yeager, B. Schumm, Jr., K. Mauritz, K. Abbey, D. Blankenship, and J. Akridge, The Electrochemical Society, Inc., Pennington, NJ (1983).

[2] Fiona M. Gray, Solid Polymer Electrolytes, VCH, New York, NY (1991). 
[3] Perfluorinated Ionomer Membranes, edited by A. Eisenberg and H. L. Yeager, Amer. Chem. Soc. Symposium Series No. 180, Washington, D.C. (1982).

[4] Solid State Batteries, edited by C. A. C. Sequeira and A. Hooper, NATO ASI Series E, No. 101, Dordrecht (1985).

[5] Solid State Protonic Conductors for Fuel Cells and Sensors, edited by John B. Goodenough, Johs. Jensen, and Michel Kleitz, Odense University Press (1983).

[6] K. Prater, "The Renaissance of the Solid-Polymer Fuel Ce11," J. Power Sources, 29, 239-250 (1990).

[7] H. A. Liebhafsky and E. J. Cairns, Fuel Cells and Fuel Batteries, John Wiley \& Sons, Inc., New York (1968).

[8] R. A. Lemons, "FueI Cells for Transportation," J. Power Sources, 29, 251-264 (1990).

[9] A. J. Appleby and F.R. Foulkes, Fuel Cell Handbook, Van Nostrand Reinhold, New York (1989).

[10] Edson A. Ticianelli, Charles R. Derouin, and Supramaniam Srinivasan, "Localization of platinum in low catalyst loading electrodes to attain high power densities in SPE fuel cells," J. Electroanal. Chem., 251, 275 (1988).

[11] J. Giner, and C. Hunter, "The Mechanism and Operation of the Teflon-Bonded Gas Diffusion Electrode: A Mathematical Model," J. 
Electrochem. Soc., 116, 1124-1130 (1969).

[12] Edson A. Ticiane1li, Charles R. Derouin, A. Redondo, and S. Srinivasan, "Methods to Advance Technology of Proton Exchange Membrane Fuel Cells," J. Electrochem. Soc., 135, 2209-2214 (1988).

[13] Structure and Properties of Ionomers, edited by Michul Pineri and Adi Eisenberg, NATO ASI Series C: Mathematical and Physical Sciences Vol. 198, D. Reidel Publishing Company, Dordrecht (1989).

[14] T. D. Gierke and W. Y. Hsu, "The Cluster-Network Model of Ion Clustering in Perfluorosulfonated Membrane, " Perfluorinated Ionomer Membranes, edited by A. Elsenberg and H. L. Yeager, Amer. Chem. Soc. Symposium Series No. 180, Washington, D.C. (1982).

[15] Carl Berger, ed., Handbook of Fuel Cell Technology, Prentice-Hall, Inc., Englewood Cliffs, New Jersey (1968).

[16] J. L. Fales, N. E. Vanderborgh, and P. Stroeve, "The Influence of Ionomer Channel Geometry on Ionic Transport," in Diaphragms, Separators, and Ion-Exchange Membranes, proceedings volume 86-13, edited by J. W. Van Zee, R. E. White, K. Kinoshita, and H. S. Burney, The Electrochemical Society, Pennington, NJ (1986).

[17] A. B. LaConti, A. R. Fragala, and J. R. Boyack, "Solid Polymer Electrochemical Cells: Electrode and Other Materials Consideration," in Proceedings of the Symposium on Electrode Materials and Processes for Energy Conversion and Storage, edited by J. D. E. 
McIntyre, S. Srinivasan, and F. G. Will, The Electrochernical Sociery, Inc., Pennington, NJ (1977).

[18] Supramaniam Srinivasan, "Fuel Cells for Extraterrestrial. and Terrestrial Applications," J. Electrochem. Soo., 136, 41C (1989).

[19] Carl Wagner, "The Electrochemistry of Ionic Crystals," J. Electrochem. Soc., 99, 346C (1952). 


\section{Chapter 2}

\section{Theory of Transport in Polymer Electrolytes}

\subsection{Introduction}

In concentrated solutions, such as some polymer electrolytes, the distinction between the solvent and solute is unclear. Further. more, there can be significant interactions among all of the species in polymer electrolytes, whereas in dilute solutions, only interactions between the minor species and the solvent are considered. Mass transfer in these polymeric systems cannot be adequately described by dilute solution theory. Newman [1] discusses further the limitations of dilute solution theory and the motivation for the use of concentrated solution theory.

Modeling of transport in the solid-polymer-electrolyte is based on the concepts of irreversible thermodynamics and concentrated solution theory. Irreversible thermodynamics deals with non-equilibrium processes. The objective is to relate the production of entropy to the physical phenomena occurring in the system [2]. Rather than the study of equilibrium states, we are concerned with stationary states, which correspond to minimum entropy production consistent with the boundary conditions imposed. Within the context of concentrated solution theory, the interactions between the species are accounted for in a straightforward manner. Coupling of transport phenomena, the electroosmotic effect and streaming potential, for example, arise naturally. 
The concentration scale used in later chapters is the mole fraction. While this is familiar to chemists, it introduces the molecular weight of the membxane or polymer, which may be a capricious choice. This problem is riscussed in more detail later in this chapter. In principle, any concentration scale could be used. The mole fraction scale is conceptually easy to use; in retrospect, however, it may not be the most appropriate one for some polymer sys. teins .

In concentrated solutions, Fick's law is replaced with the equation of multicomponent diffusion

$$
a_{i}=\sum_{j \neq i}^{n} K_{i j}\left(v_{j}-v_{i}\right)=R T \sum_{j \neq i} \frac{c_{i}{ }^{c} j}{c D_{i j}}\left(v_{j}-v_{i}\right)
$$

where $d_{1}$ is a general driving force for transport. $k_{i i}$ is not defired, and from Newton's third law or the Onsanger reciprocal relations

$$
K_{i j}=K_{j i}
$$

\subsection{Driving Forces}

Bennion [3] showed how to determine the appropriate driving forces for a membrane system. Although it may be difficult to characterize the state of a polymer system near its glass transition temperature, we consider the solid-polymer-electrolyte to be an elastic solid and not a fluid. The polymer membrane is able to resist forces that tend to deform it. Bennion's approach is applied to the 
solid-polymer-electrolyte system.

The driving forces should be zero at equilibrium, and furthermore, the Gibbs-Duhem equation,

$$
\sum_{i} c_{i} \nabla \mu_{i}=\nabla p-\frac{S}{V} \nabla T
$$

must be satisfied. Hirschfelder et al. [4] and Bird et al. [5] developed the following driving force,

$$
\mathrm{d}_{i}=c_{i}\left\{\nabla \mu_{i}+\bar{s}_{i} \nabla T-\frac{M_{i}}{\rho} \nabla p-\mathrm{x}_{i}+\frac{M_{i}}{\rho} \sum_{j} \mathrm{x}_{j} c_{j}\right\} .
$$

In principle we should expect coupling between all tensors of the same order. Thermal diffusion and the soret effect, for example, are not considered. These effects are generally small, and furthermore we are considering an isothermal system. $x_{i}$ is an external or whole body force per mole, such as gravity. For our purposes electrostatic forces are included in the electrochemical potential of the species, $\mu_{i}$, and therefore do not appear as external forces in equation 4.

One could consider a membrane with a pressure difference across it and steady, bulk flow of water through the membrane. Since the membrane is stationary, the net force on any volume element must be zero. There are no body forces acting on either the water or the hydrogen ions; therefore, at constant temperature,

$$
\mathrm{x}_{m}=\frac{M_{m}}{\rho_{m}} \nabla p
$$


and

$$
c_{m} \mathrm{x}_{m}=\nabla p
$$

In words, force from the presure gradient is transferred through the various frictional interactions to the membrane, which is supported by the electrodes of the fuel cell. This relationship is used in equation 4. The appropriate isothermal driving forces are:

for hydrogen and water

$$
\mathrm{d}_{i}=c_{i} \nabla \mu_{i}
$$

and for the membrane

$$
\mathrm{d}_{m}=c_{m} \nabla \mu_{m}-\nabla p
$$

For $n$ species, $n$ equations of the form of equation 1 . may be written, but they are not all independent. From the Gibbs-Duhem relation, the sum on the left side of equation 1 is zero. The sum on right side is zero because of equation 2. Substitution of the driving forces into equation 1 yields the transport equations for the ionically conducting polymer of the hydrogen/oxygen fuel cell:

$$
\begin{aligned}
& c_{+} \nabla \mu_{+}=K_{0+}\left(v_{0}-v_{+}\right)+K_{m+-}\left(-v_{+}\right), \\
& c_{0} \nabla \mu_{0}=K_{+0}\left(v_{+}-v_{0}\right)+K_{o m}\left(-v_{0}\right),
\end{aligned}
$$

and 


$$
c_{m} \nabla \mu_{m}-\nabla P=K_{+m}\left(v_{+}\right)+K_{o m}\left(v_{o}\right)
$$

In the above equations we have used the membrane velocity, which is zero, for the reference velocity; The subscripts,$+ m$, and $o$ refer to hydrogen ions, membrane, and water respectively.

An alternative approach has been presented by. Paul [6] [7], who treats the membrane, solutes, and solvent as a homogeneous liquid. Paul then hypothesizes high surface tension which allows the liquid to resist deformation. This leaves one with the unsatisfactory condition that for a supported membrane a whole body force must act on both the solute and solvent as well as the polymer.

\subsection{Transport Properties}

In general, for $n$ species there are $\frac{1}{2} n(n-1)$ independent trans. port properties necessary to characterize the system. There are three species in the separator of a solid-polymer-electrolyte fuel cell, and therefore three independent transport properties are required. With the electrochemical potential as the driving force, equations 9 and 10 can be inverted to give

$$
\mathbf{N}_{+}=c_{+} v_{+}=-L_{++} c^{2} \nabla \mu_{+}-L_{+0} c_{+} c_{0} \nabla \mu_{0},
$$

and

$$
N_{0}=c_{0} v_{0}=-L_{0+} c_{0} c_{+} \nabla \mu_{+}-L_{00} c_{0}^{2} \nabla \mu_{0} .
$$

The $L_{i j}$ 's $\left(-L_{j i}\right)$ are related directly to the $K_{i j}$ : 


$$
\begin{aligned}
& L_{o+}=\frac{K_{+o}}{K_{+o} K_{o m}+K_{+o} K_{+m}+K_{+m} K_{o m}}, \\
& L_{o o}=\frac{K_{+o}+K_{+m}}{K_{+o} K_{o m}+K_{+o} K_{+m}+K_{+m} K_{o m}},
\end{aligned}
$$

and

$$
L_{++}=\frac{K_{+o}+K_{o m}}{K_{+o} K_{o m}+K_{+o} K_{m m}+K_{+m} K_{o m}} .
$$

Conversely, the frictional coefficients can be expressed in terms of $L_{i j} s$ :

$$
\begin{aligned}
& K_{+0}=\frac{L_{+0}}{L_{00} L_{++}-L_{+0}{ }^{2}}, \\
& K_{m o}=\frac{L_{++}-L_{+0}}{L_{00} L_{++}-L_{+o} 2},
\end{aligned}
$$

and

$$
K_{+m}=\frac{L_{o 0}-L_{+0}}{L_{00} L_{++}-L_{+o}^{2}} .
$$

If experiments can be devised to measure the $L_{i j}$ 's, then the multicomponent diffusion coefficients can be estimated as functions of water content within the membrane. This concept is the $s$ me as the orthogonal experiments described by Bennion and Pintauro [8], $[9]$. 
First, consider the case where $\nabla \mu_{0}=0$. The conductivity of the membrane is measured by standard alternating-current techniques. From equation 1.2 and the definition of conductivity

$$
-I_{+}=-\frac{\kappa}{F^{2} c^{2}}
$$

where the potential is defined by $\mu_{+}=F \Phi$.

Next consider that a small curxent is passed through the membrane, but $\nabla \mu_{0}$ is equal to zero. The flux of water through the membrane is measured. If $\xi$ is the number of water molecules carried across the membrane with each hydrogen ion, then

$$
-L_{o+}=\frac{-\kappa \xi}{F^{2} c_{+} c_{0}} .
$$

Finally considex a case where no current is passed, $N_{+}=0$, but water flows because of a gradient in its chemical potential. The rate of diffusion is given by Fick's law

$$
\mathrm{J}_{0}=-\alpha \nabla \mu_{0}
$$

The flux of water is relative to the velocity of the membrane and can be related to a measurable diffusion coefficient (see appendix $A$ ). Equations 12 and 13 reduce to

$$
L_{00}=-\frac{1}{c_{0}^{2}}\left(\alpha+\frac{\kappa \xi^{2}}{F^{2}}\right)
$$


Thus, from three measurements the needed transport properties are determined.

We can now write expressions for the flux of water and hydrogen ions in terms of measurable quantities. Equations 12 and 13 become

$$
1=-\frac{\kappa}{F} \nabla \mu_{+}-\frac{\kappa \xi}{F} \cdot \nabla_{\mu_{0}}
$$

and

$$
\mathrm{N}_{0}=-\frac{\kappa \xi}{F^{2}} \nabla \mu_{+}-\left(\alpha+\frac{\kappa \xi^{2}}{F^{2}}\right) \nabla \mu_{0} .
$$

Or with the potential defined by $F \Phi=\mu_{+}$,

$$
1--\kappa \nabla \Phi-\frac{\kappa \xi}{F} \nabla \mu_{0}
$$

and

$$
N_{0}=-\frac{\alpha \xi}{F} \nabla \Phi-\left(\alpha+\frac{\kappa \xi^{2}}{F^{2}}\right) \nabla \mu_{0} .
$$

The above equations completely describe transport fin the polymer. If we want to put them in the form of the Stefan-Maxwell equations, we must introduce a concentration scala.

\section{2,4 Concentration scale}

There is a variety of driving forces for diffusion, such as gradients in mole fraction, mass fraction, concentration, and chemical potential. Some of these formulations depend on the molecular weight 
of the polymer, which can be large compared to the molecular weight of other species and may be poorly defined. We wish to investigate the effect of the molecular weight on the different driving forces and the binary interaction coefficients, $D_{i j}$.

The driving force derived earlier, $c_{i} \nabla \mu_{i}$, is per unit volume and therefore independent of the molecular weight of the species. Similarly, the right side of equation 1 represents a frictional force per unit volume times the velocity of the species, and is also independent of the molecular weight. Although it may not be obvious from the expressions for $L_{i j}$ 's given in equations 20,21 , and 23 , these too are independent of molecular weight. Nevertheless, we need the concentration to relate molar fluxes to their velocity.

The binary interaction coefficients used in the Stefan-Maxwell formulation are related to the frictional coefficients by

$$
D_{i j}=\frac{R T c_{i} c_{1}}{c K_{i j}} .
$$

The $D_{i j}$ 's, of course, depend on the cholce of molecular weight. These binary interaction coefficients were defined in the hope that they would be more nearly constant with concentration. $c_{i}{ }_{j}$ represents the collision frequency of molecules $i$ and $j$. Whereas this is reasonable for gases and dilute electrolytes, there does not appear to be a sound basis for this approach with polymer electro. lytes, particularly when the molecular weight of the polymer may be ill-defined. 
The above transport equations are phenomenological and could be used without the introduction of molecular weight. However, the mole fraction scale was used in much of the analysis. We now examine the implications of this driving force. Consider species $A$, a highmolecular-weight species, and $B$, a small molecule. Let $c_{A}$ be the concentration of species $A$ when we regard the molecular weight to be $M_{A}$. If we regard the molecular weight to be $M_{A}^{\prime}$, then the concentr:tion of $A$ is

$$
c_{A}=c_{A} \frac{M_{A}}{M_{A}^{\prime}}
$$

We know that the $K_{A B}$ is independent of molecular weight; therefore

$$
K_{A B}=\frac{c_{A}{ }^{c} B}{C D_{A B}}=\frac{c_{A}^{\prime}{ }^{c} B}{C^{\prime} D_{A B}^{\prime}} .
$$

This nay be rearranged to give

$$
D_{A B}^{\prime}=D_{A B} \frac{M_{A}}{x_{A} M_{A}+x_{B} M_{A}^{\prime}}
$$

Thus, $D_{A B}^{\prime}$ approaches zero as $M_{A}^{\prime} \rightarrow \infty$. Although this formulation will work, it has this troubling consequence. In principle, the choice of molecular weight in these phenomenological equations is arbitrary. Although a different concentration scale (such as mass fraction) may be more appropriate, it is not investigated further here. 


\subsection{Bulk Hydrodynamic Flow}

As in the development of the driving forces, we consider bulk, steady hydrodynamic flow under a pressure gradient. The membrane is able to resist a pressure gradient, and stress builds up in the membrane. From equations 9.11 with the current equal to zero, the velocity of water may be expressed as

$$
v_{o}=\frac{c_{m} \nabla \mu_{m}}{k_{m o}}-\frac{\nabla_{p}}{K_{m o}}-\frac{-c_{o} \mu_{o}}{k_{o t}+K_{o m}}
$$

If the gradient in chemical potential of water is due solely to a pressure gradient, from the thermodynamic relation

$$
\nabla \mu_{0}=\bar{v} \nabla p
$$

equation 32 can be written as

$$
v_{0}-\frac{k}{\mu} \nabla p
$$

where $k$ is the permeability and $\mu$ the viscosity of water.

There are two points to note. First, the multicomponent diffusion equations account for hydrodynamic flow, and superposing additional equations for the flow of water is incorrect. When using concentrated solution theory and these driving forces, additional equations to account for hydrodynamic flow are unnecessary; all the mutual interactions between species are included in equation 1. Second, equation 34 is similar to either flow through porous media or 
Poiseuille flow through a pore.

\subsection{Equilibrium with Water and Hysteresis}

The most important variable in determining the properties of the membrane is its equivalent weight. The amount of water absorbed will depend on the equivalent weight, the ion form, the fluid it is in contact with, and the pretreatment of the membrane. The equilibrium water content in a Nafion 117 membrane is shown in figure 1 . The data are reproduced from Zawodzinski et al. [10]. The equilibrium curve does not show a plateau at high relative humidities as would be expected for a Langmuir isotherm, for example.

A membrane that has received identical pretreatment can have a different water content when exposed to liquid water as opposed to saturated water vapor [11]. Although no careful study of this phenomenon has been attempted, clearly this is not expected from thermodynamics. This isotherm resembles equilibrium-moisture curves for some cloths, for example, silk [12]. We can consider that water in the membrane that exerts a vapor pressure less than that of pure liquid water is bound water. It will have a lower vapor pressure due to capillary forces, chemical forces such as hydration effects, and physical adsorption. The condition at which the membrane is in equilibrium with saturated air is the fiber-saturation point. Additional water in the membrane above the fiber-saturation point may be considered unbound water. 


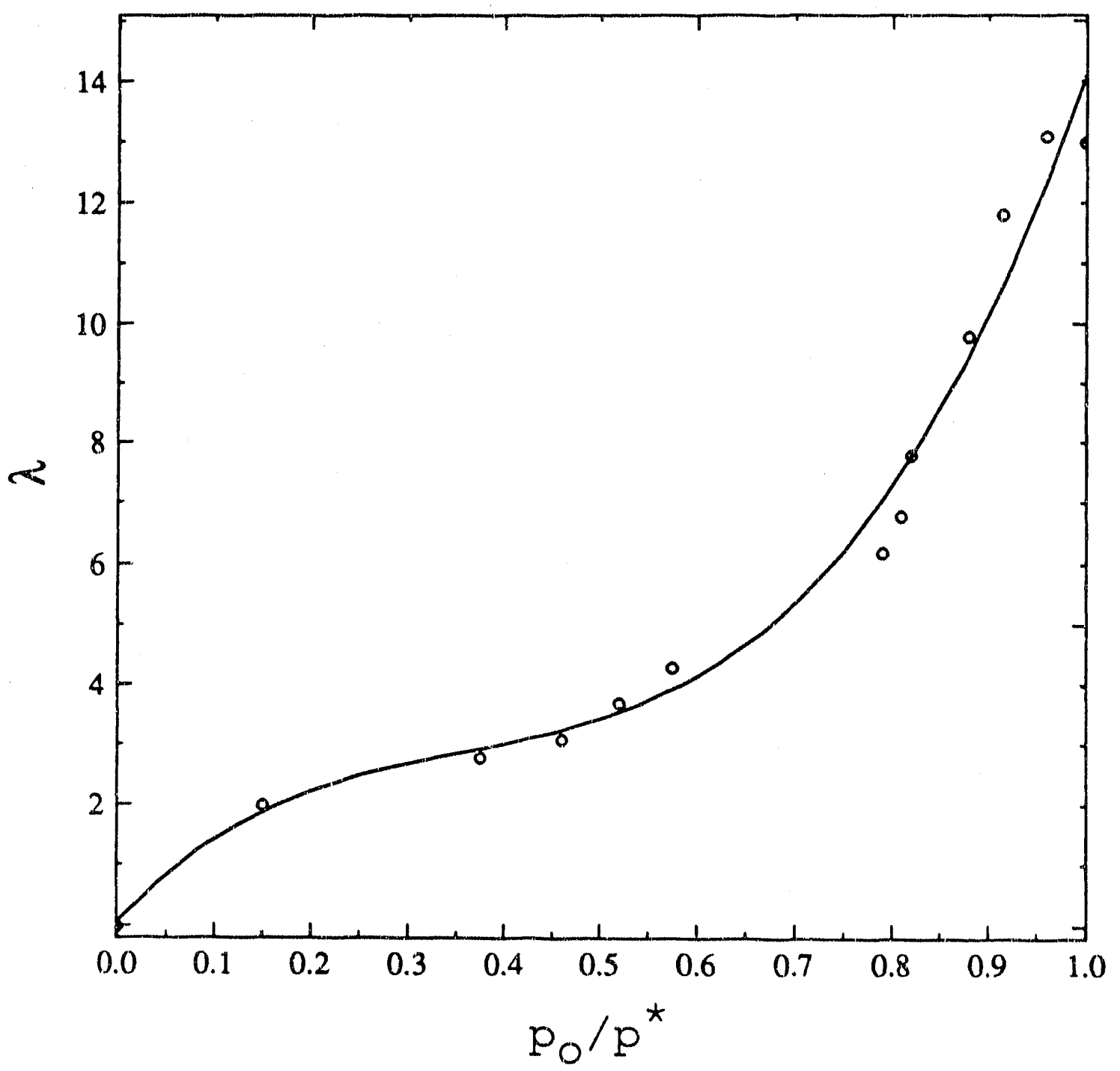

Figure 1. Absorption isotherm for Nafion 117 at $30^{\circ} \mathrm{C}$. Measured by Zawodzinski et a].[10]. 
Additionally, perfluorinated ionomeric membranes show hysteresis. The amount of water sorbed by the membrane in contact with a fixed activity of water will depend on its pretreatinent. For example, a membrane pretreaced by boiling in water will retain more water than a membrane that is not pretreated. We should expect that the pretreatment will affect the transport propertiles as well.

\section{List of Symbols}

$\begin{array}{ll}c_{i} & \text { total concentration, mol/l } \\ c_{i} & \text { concentration of species } i, \mathrm{~mol} / \mathrm{l} \\ \mathrm{d}_{i} & \text { driving force for transport of species } \mathrm{i}, \mathrm{N} / \mathrm{m}^{3} \\ D_{i j} & \text { binary-interaction coefficient, } \mathrm{cm}^{2} / \mathrm{s} \\ F_{i} & \text { Faraday's constant, } 96,487 \mathrm{C} / \mathrm{eq} \\ \mathrm{J} & \text { flux of species } \mathrm{i}, \mathrm{mol} / \mathrm{cm}^{2} \cdot \mathrm{s} \\ K_{i j} & \text { hydraulic permeability, } \mathrm{m}^{2} \\ L_{i j} & \text { frictional coefficient defined by equation } 1 \\ M_{i} & \text { coefficient defined in equations } 14-16 \\ n & \text { molecular weight of species } i, \mathrm{~g} / \mathrm{mol} \\ \mathrm{N}_{i} & \text { number of species } \\ p & \text { molar flux of species } i, \mathrm{~mol} / \mathrm{cm}^{2} \cdot \mathrm{s} \\ R & \text { pressure, bax } \\ S & \text { universal gas constant, } 8.3143 \mathrm{~J} / \mathrm{mol} \cdot \mathrm{K} \\ \bar{S}_{i} & \text { entropy, J/K }\end{array}$




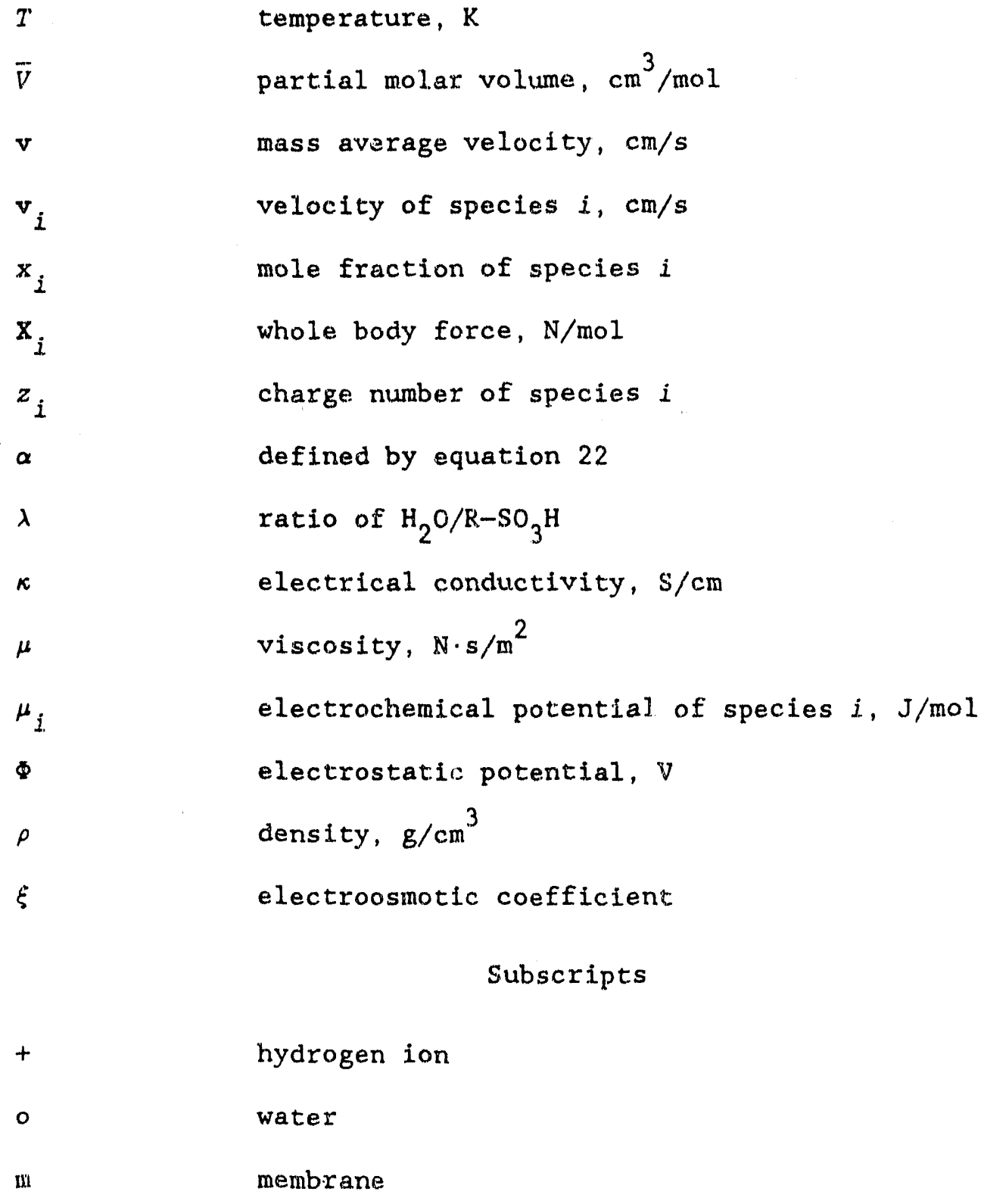

References

[1] John Newman, Electrochemical Systems, 2nd edition, Prentice-Hall Inc, Englewood Cliffs, N.J. (1991).

[2] S. R. de Groot and P. Mazur, Non-Equilibrium Thermodynamics, Dover Publications, New York (1984). 
[3] D. N. Bennion, Mass Transport of Binary Electrolyte Solutions in Membranes, Water Resources Center Desalination Report No. 4, Report No. 66-17, University of California, Los Angeles, CA (1966).

[4] J. O. Hirschfelder, C. F. Curtiss, and R. B. Bird, Molecular Theory of Gases and Liquids, John Wiley \& Sons, Inc., New York (1954).

[5] R. Byron Bird, Warren E. Stewart, and Edwin N. Lightfoot, Transport Phenomena, John Wiley \& Sons, Inc., New York (1960).

[6] D. R. Paul, "The Role of Membrane Pressire in Reverse Osmosis," J. of Applied Polymer Science, 16, 771 (1972).

[7] D. R. Paul and J. D. Paciotti, "Driving Force for Hydraulic and Pervaporative Transport in Homogeneous Membranes," J. of Polymer Science, 13, 1201 (1975).

[8] P. N. Pintauro and D. N. Bennion, "Mass Transfer of Electrolytes in Membranes. 1. Development of Mathematical Model and 2. Determination of $\mathrm{NaCl}$ Equilibrium and Transport Parameters for Nafion," Ind. Eng. Chern. Fund., 23, 230 (1984).

[9] P. N. Pintauro, Ph. D. dissertation, University of Califor. ria, Los Angeles, CA (1980).

[10] T. Zawodzinski, M. Neeman, L. Sillerud, and S. Gottesfeld, "Determination of Water Diffusion Coefficients in Perfluorosulfonated Ionomeric Membranes," J. Phys. Chem., 95, 6040.6044 (1991). 
[11] T. A. Zawodzinski, F. Garzon, V. T. Smith, and S. Gottesfeld, "Thermodynamic and Kinetic Studies of Water in Perfluorinated Ionomers," Extended Abstracts of the 178th Society Meeting (The Electrochemical Society, Fall 1990), Abstract No. 117.

[12] Warren L. McCabe, Julian C. Smith, and Peter Harriot, Unit Operations of Chemical Engineering, McGraw-Hill. Book Company, New York (1985). 
Chapter 3

Modeling

\subsection{Introduction}

Solid-polymer-electrolyte fuel cells are fabricated by hotpressing two gas-diffusion electrodes onto a thin pulymer membrane above its glass transition temperature [1]. This is shown schematically in figure 1. Leddy and Vanderborgh [2] characterize the membrane-electrode interface and present a photomicrograph of the region. There are three phases present: a solid phase consistirig of the porous graphite electrode and platinum catalyst, the solidpolymer-electrolyte, and a gas phase. The solid polymer serves both as the separator and the electrolyte. Descriptions of gas-diffusion electrodes and models can be found in [3], [4], and [5].

We consider a membrane-electrode assembly of a fuel cell operating at steady state on air and reformed methanol. The fuel and air streams are heated and humidified prior to entering their respective channels where they are consumed in chemical reactions. Hydrogen gas diffuses through the porous electrode and reacts at the anode in a three-phase region containing polymer electrolyte, gaseous reactants, and carbon matrix including platinum catalyst, to form hydrogen ions. Oxygen passes through the gas-diffusion electrode to the cathodic reaction zone. At the cathode, the hydrogen ions react with oxygen at electrocatalyst sites to form water. 

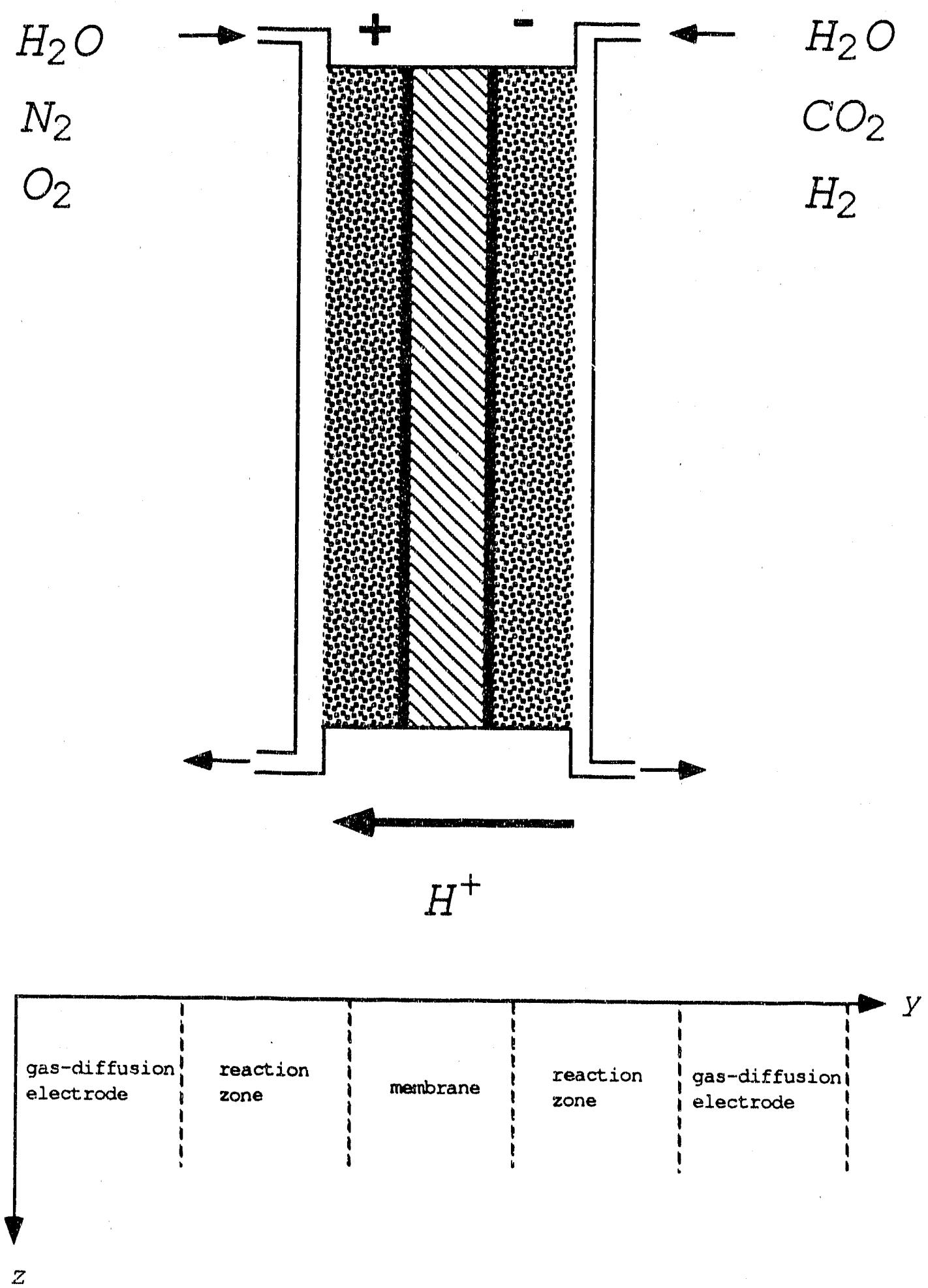

Figure 1. Membrane and electrode assembly. Five regions of the model are shown, not to scale. 
Recently, Kimble and White [6] presented a detailed model of an alkaline fuel cell; solid-polymer-electrolyte fuel-cell models have been developed by Bernardi and Verbrugge [7] [8] [9] and by Springer et al. [10] Our model contrasts with other models mainly by: 1) use of concentrated solution theory, 2) consideration of a twodimensional membrane-electrode assembly, and 3) accounting for thermal effects.

In the previous chapter we derived equations for transport in the solid polymer electrolyte. Even though we can consider the polymer to be a binary electrolyte, the treatment that follows is general, allowing for an arbitrary number of species, and homogeneous and heterogeneous chemical reactions. similarly, in the gas phase, in the absence of chemical reactions, analytic solutions of the Stefan-Maxwell equations are possible for a three-component system with constant diffusion coefficients. Nevertheless, we elected to keep the analysis general.

\section{2 Gas Phase}

Transport in the gas phase is described by the Stefan-Maxwe11 equation. For m species,

$$
\frac{\mathrm{d}_{i}}{R T}=\sum_{j \neq i}^{m} \frac{x_{i} \mathrm{~J}_{j}^{-\mathrm{x}_{j} \mathrm{~J}_{i}}}{c D_{i j}} .
$$

The molar flux is given by,

$$
\mathrm{J}_{j}=c_{i}\left(\mathrm{v}_{i}-\mathrm{v}_{\text {ref }}\right) .
$$


One is free to choose the reference velocity. For the solidpolymer-electrolyte fuel cell, the most convenient reference velocity is the velocity of the nondiffusing gases, carbon dioxide and nitrogen, which is zero. This choice is consistent with the reference velocity of the electrolyte;

$$
\mathbf{v}_{\text {ref }}=\mathbf{v}_{\mathrm{CO}_{2}}-\mathbf{v}_{N_{2}}=\mathbf{v}_{\text {mem }}=0
$$

Assuming ideal-gas behavior, the Stefan-Maxwell equation becomes

$$
\frac{\nabla \mathrm{x}_{i}}{R T}-\sum_{j \in i}^{m} \frac{\mathrm{x}_{i} \mathrm{~J}_{j}-\mathrm{x}_{j} \mathrm{~J}_{i}}{D_{i j} P} .
$$

For $m$ species, there are only $m-1$ independent equations of the form of equation (1). From the Gibbs-Duhem relationship, the sum of the left side of equation 1 is zero. The sum of the right side is zero because of the Onsager reciprocal relations. The relation among molar fluxes is

$$
\mathrm{J}_{\mathrm{CO}_{2}}-\mathrm{J}_{N_{2}}=0
$$

The m-1 equations of the form of equation 4 and equation 5 can be written in matrix form

$$
\frac{1}{R T} \underline{\nabla x}_{\dot{I}}=\underline{b} \underline{J}
$$

The matrix can be inverted to express $J_{i}$ in terms of the driving forces. A material balance on species $i$ gives 


$$
\frac{\partial\left(\epsilon c_{i}\right)}{\partial t}=-\nabla \cdot J_{i}+a j_{i n}+\sum_{\ell=1}^{1} R_{i, \ell} .
$$

$R_{i, l}$ is the homogeneous reaction rate of species $i$ for the $l$ th reaction, and is the spectfic interfacial area. The pore-wall flux density of species $i, j_{i n}$ is an average over the interfacial area between the gas phase, and the electrode and electrolyte phases. The relationship anong mole fractions is

$$
\sum_{i=1}^{m} x_{i}=1
$$

Thus, equations $4,5,7$, and 8 describe transport in the gas phase.

\subsection{Electrolyte Phase}

The transport model is based on the theory developed in the previous chapter. For $n$ species in the electrolyte, the multicomponent diffusion equation is

$$
a_{j}=\sum_{j=i}^{n} x_{i j}\left(v_{j}-v_{i}\right) .
$$

Equation (9) can be written in terms of $D_{i j}$, and then has a form similar to that of the Stefan-Maxwell equation

$$
\frac{d}{R T}-\sum_{j=i}^{n} \frac{c_{i} c j}{c D_{i j}}\left(v_{i}-v_{j}\right)
$$

The flux of species is 


$$
J_{i}=c_{i}\left(v_{i}-v_{\text {ref }}\right) .
$$

For the solid-polymer-electrolyte system, the reference velocity is chosen to be the membrane velocity. Equation 10 can be written in terms of molar fluxes;

$$
\frac{d_{i}}{R T}-\sum_{j \in i}^{n} \frac{x_{i} \mathrm{~J}_{j}-\mathrm{x}_{j} \mathrm{~J}_{i}}{c D_{i j}}
$$

A material balance on species $i$ is identical to equation 7 . In the solid-polymer-electrolyte, there are no homogeneous chemical reactions. However, the homogeneous reaction terms were included in the material balance to remain general. For example, in some polymex electrolytes ion pairing may be important [11].

The separator of a solid-polymer-electrolyte fuel cell contains three species: the membrane with the bound sulfonic acid groups, hydrogen ions, and water. The proper driving forces, $d_{i}$, are as follows (see chapter 2):

for hydrogen and water

$$
d_{i}=c_{i} \nabla \mu_{i},
$$

and for the membrane

$$
\mathrm{d}_{m}=c_{m} \nabla \mu_{m}-\nabla p
$$

In the electrolyte, there is an additional dependent variable, $\Phi$, the electric potential, which we must introduce as a driving force 
for transport. Newman discusses the various potentials we could use [12]. We could, for example, define the potential in terms of a reference electrode or the "quasi-electrostatic potential" of Smyr]. and Newman [13]. In the latter case the potential is defined in terms of the hydrogen ion,

$$
\mu_{+}=R T \ln c_{+}+z_{+} F \Phi
$$

The gradient of the electrochemical potential of each of the species is

$$
\nabla \mu_{i}-R I \nabla \ln c_{i}+z_{i} F \nabla \Phi+R T \nabla\left(\ln f_{i}-\frac{z_{i}}{z_{+}} \ln f_{+}\right) .
$$

In the above equation, only the activity of neutral species or neutral combinations of ionic species is needed, and everything is well-defined. For the Nafion inembrane of the solidnpolymerelectrolyte fuel cell, there is only one compositional variable. Using the thermodynanic data of Zawodzinski et al. [1.4], for example, we could express the gradient in electrochemical potential of each species in terms of equation 16.

In the modeling, for simplicity, the electrochemical potential of each species was taken to be

$$
\nabla \mu_{i}=z_{i} F \nabla \Phi+R T-\frac{\nabla x_{i}}{x_{i}}
$$

Here the choice of molecular weight implicitly affects the assumption 
for the activity coefficients. We took the molecular weight to be equal to the equivalent weight for the membrane. We also assumed the pressure to be a constant.

The relationship among mole fractions is

$$
\sum_{i=1}^{n} x_{i}=1
$$

The equstion of electroneutrality is

$$
\sum_{i=1}^{n} z_{i} x_{i}=0
$$

Therefore, combining equations 12 with the appropriate driving forces, together with equations 7,18 , and 19, we have described transport in the electrolyte.

\subsection{Chenical Reactions}

For either anodic or cathodic processes within the fuel cell the following sequence is necessary: 1) mass transfer of gaseous reactants to the interfacial region, 2) mass transfer from the gas pore to electrolyte, 3) absorption of the reactant into the electrolyte, 4) mass transfer to catalytic site, 5) adsorption onto a catalyst sj.te, and 6) electron-transfer reaction.

We need to relate the transfer current of species through the interface between the gas and electrolyte phase. The rate of reaction per unit volume is 


$$
a_{\text {in }}-r_{i}
$$

where $j_{i n}$ is the pore wall flux. Each reaction is written as an elementary reaction

$$
\sum_{i} s_{i} M_{i}^{z} \rightleftarrows n e^{-} .
$$

For electron transfer reactions, \& Butler-Volmer rate expression is used;

$$
\frac{r}{a}=\frac{i}{n F}=k_{a} \prod c_{m}{ }^{s} \exp \left[\frac{(1-\beta) n F}{R T} V\right]-k_{c} \prod_{n}{ }^{-s_{n}} \exp \left(-\frac{\beta n F}{R T} V\right)
$$

where the first product is over species with positive $s_{i}$, and the second over species with negative $s_{i}, V$ is the difference in potential between the metal and electrolyte,

$$
v-\Phi_{1}-\Phi_{2}
$$

For the solid-polymer-electrolyte fuel cell, we consider two electrachemical reactions and one evaporation reaction:

$$
\begin{gathered}
\mathrm{H}_{2(\mathrm{~g})} \rightleftarrows 2 \mathrm{H}^{+}+2 \mathrm{e}^{-}, \\
\mathrm{O}_{2(\mathrm{~g})}+4 \mathrm{H}^{+}+4 \mathrm{e}^{--} \rightleftarrows 2 \mathrm{H}_{2} \mathrm{O}(\mathrm{g})^{\prime}
\end{gathered}
$$

and 


$$
\mathrm{H}_{2} \mathrm{O}(\ell) \rightleftarrows \mathrm{H}_{2} \mathrm{O}(\mathrm{g})
$$

We assumed that the rate of reaction could be expressed in terms of the bulk concentration of each species in their respective phases, neglecting the details of steps 2 to 5 above.

The oxidation of hydrogen, for example, is known to proceed by the Tafel-Volmer sequence [15],

$$
\begin{gathered}
\mathrm{H}_{2}+2 \mathrm{M} \rightleftarrows 2 \mathrm{MH} \\
\mathrm{MH} \rightleftarrows \mathrm{M}+\mathrm{H}^{+}+\mathrm{e}^{-}
\end{gathered}
$$

with the Tafel (second) step rate limiting, We, however, have assumed that for the hydrogen reaction, we can write

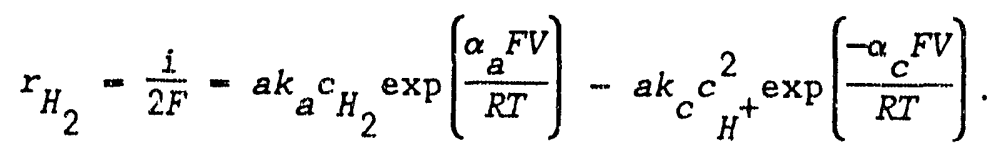

\subsection{Boundary Conditions}

At the two ends of the fuel-cell assembly, $y=0$ and $y=L 5$, the mole fraction of the gaseous species are determined from a material balance on species $i$ in the $z$ direction of the gas channel (see the next section;. At a phase boundary, the fluxes of the species that exist only within that phase are set to zero. At L4, for example, the fluxes of electrolyre species (water in the polymer, hydrogen ions, and membrane) are zero. Where there is a discontinuity in the porosity, the concentration and superficial fluxes are continuous. Therefore, at 23 , the fluxes of gas species axe set to zero, and the 
concentration and superficial fluxes of electrolyte species are matched. The potential of the anode is set to zero, and the potential of the cathode is specified and assumed constant, thereby setting the cell potential $V$ as a constant in $z$ and time.

\section{$3.6 \mathrm{z}$-integration}

The problem is two-dimensional, but the large aspect ratio allows us to consider transport in the $y$ direction only in the cell sandwich (see figure 1). Although this is not strictly correct, the expected improvernents in results do not warrant the additional complexities of a two-dimensional model. We will solve the transport in the $y$ direction at a given value of $z$ and integrate down the membrane-electrode assembly in the $z$ direction. The gas outside the gas-diffusion electrode is assumed to be of uniform composition in the $y$ direction. For large Péclet number, axial diffusion is neglected. A material balance on species $i$ in the direction gives

$$
F_{i}-F_{i}^{0}=\int_{0}^{z} J_{i} d z
$$

where $F_{i}$ is the molar flow rate of species $i$ in the $z$ direction and $I_{i}$ is the molar flux in the $y$ direction.

\subsection{Energy Balance}

References [12] and [16] discuss general energy balances for electrochemical systems. The work done by the system is iV. The 
rate of neat transfer to the system can be expressed by

$$
Q=-h A\left(T-T_{A}\right)
$$

Application of the first law of thermodynamics to the fuel cell gives

$$
\frac{-\Delta H}{b}-\int_{0}^{z} h\left(T-T_{A}\right) d z+\int_{0}^{z} V i d z
$$

where $b$ is the width of the membrane-electrode assembly. The enthalpy of the gas streams is

$$
H=\sum_{i}^{m} F_{i} \bar{H}_{i}
$$

Assuming no enthalpy change upon mixing and neglecting the effects of pressure, the partial molar enthalpy for the gas streams can be expressed as

$$
\tilde{H}_{i}^{*}-\tilde{H}_{i}-\tilde{H}_{i}^{*}\left(T_{r}\right)+\int_{T}^{T} C_{p} d T
$$

The overail reaction is

$$
\mathrm{H}_{2(\mathrm{~g})}+\frac{1}{2} \mathrm{O}_{2(\mathrm{~g})} \rightarrow \mathrm{H}_{2} \mathrm{O}(\mathrm{g})
$$

The change in total molar flow rate of each species can then be 
related to the electrical current, $I$, and stoichiometry.

\subsection{Numerical Approach}

The set of nonlinear, partial differential equations for the $y$ direction in the gas and electrolyte phases, together with the appropriate boundary conditions, were solved numerically. We took an approach similar to that of Tribollet and Newman [17].

Taking the divergence of equation 1 and substituting for $\nabla \cdot J_{i}$ from the material balance, equation (7), and

$$
\mathrm{J}_{i}=\sum_{k=2}^{n} b_{i k}^{-1} \frac{\mathrm{d}_{i}}{R T c}
$$

from the inversion of equation 6 gives

$$
\begin{aligned}
& \nabla \cdot \frac{d_{i}}{R T c}-\sum_{j \neq i}^{n}\left(\frac{x_{i} \sum_{l=1}^{k} a r_{j, \ell}-x_{j} \sum_{\ell=1}^{k} a r_{i, l}}{c D_{i j}}\right)+c \sum_{j \neq i}^{n} \frac{x_{i} \frac{\partial x_{j}}{\partial t}-x_{j} \frac{\partial x_{i}}{\partial t}}{c D_{i j}} \\
& -\sum_{j \neq i}^{n} \nabla \cdot \frac{x_{i}}{c D_{i j}} \sum_{k=2}^{n} b_{j k}^{-1} \frac{d_{i}}{R T c}+\sum_{j \neq j}^{n} \nabla \cdot \frac{x_{j}}{c D_{i j}} \sum_{k=2}^{n} b_{i k}^{-1} \frac{d_{i}}{R T^{\prime} c}=0 .
\end{aligned}
$$

The homogeneous reaction terms have been dropped from equation 34 , but would be similar to the heterogeneous terms.

For $n$ species, there are $n+1$ dependent variables, $n$ mole fractions, and the potential. Combining the equations for the electrolyte and the gas phase, we have $n-2$ equations of the form of equation 
30. The three additional equations are the relationship among mole fractions in the gas and in the electrolyte,

$$
\sum_{i=1} x_{i}=1
$$

and the equation of electroneutrality for the polymer,

$$
\sum_{i=1}^{n} z_{i} x_{i}=0
$$

The coupling between phases occurs due to the chemical reaction terms.

To ensure fast convergence, equation 30 must be linearized prior to casting in finite-difference form. In general, the mole fractions are not known. We must, for example, use a linearized form of equation 29. $b_{i k}^{-1}$ is evaluated with the best available mole fractions and the flux is approximated with

$$
\begin{aligned}
& J_{i} \approx \sum_{k=2}^{n} b_{i k}^{-1}\left[\nabla x_{k}+\frac{z_{k}^{F}}{R T}\left(x_{k}^{\circ} \nabla \Phi+x_{k} \nabla \Phi^{\circ}-x_{k}^{o} \nabla \Phi^{\circ}\right)\right. \\
& \left.-\Delta x_{k} \sum_{j \neq k}^{n} \frac{J_{j}^{o}}{c D_{k j}}-J_{k}^{\circ} \sum_{j \neq k}^{n} \frac{\Delta x_{j}}{C D_{k j}}\right] .
\end{aligned}
$$

Newman [12] gives more details of solving systems of differential. equations. The subroutine BAND, a generalization of the Thomas method for the solution of tridiagonal matrices, was used to solve the equations simultaneously. 
Because the dimensions of the adjoining regions may be vastly different, one would like to vary the mesh spacing at phase boundaries. Equation 30 is not convenient because it does not allow the mesh spacing to be varied and still be accurate to order $h^{2}$ maintaining a tridiagonal matrix. Fan and White [18] give a general algorithm to circumvent this problem. Our approach was to replace equation 30 with an integrated version of the material balance at the junction of two regions. This allows the mesh spacing to be set independently in each region. A material balance on species $i$ gives

$$
\left.J_{i}\right|_{j+h^{\prime} / 2}-\left.J_{i}\right|_{j-h / 2}=\frac{h}{2} \sum_{\ell} a r_{i, \ell}+\frac{h^{\prime}}{2} \sum_{\ell} a r_{i, \ell},
$$

where $h$ and $h^{\prime}$ are the mesh spacings in the two adjacent regions. To evaluate the rate of reactions at $j-h / 4$ and $j+h^{\prime} / 4$, the variables are averaged as $1 / 4(j-1)+3 / 4(j)$. Again these equations were linearized, cast into finite-difference form, and solved numerically.

\section{List of Symbols}

$a$

$A$

$b$

$\underline{b}$

bik

$c$ specific interfacial area, $\mathrm{m}^{2} / \mathrm{m}^{3}$

area for heat transfer, $\mathrm{m}^{2}$

width of channel, $m$

matrix defined by equation ( 6 )

element of matrix $\underline{b}$

total concentration, $\mathrm{mol} / \mathrm{m}^{3}$ 


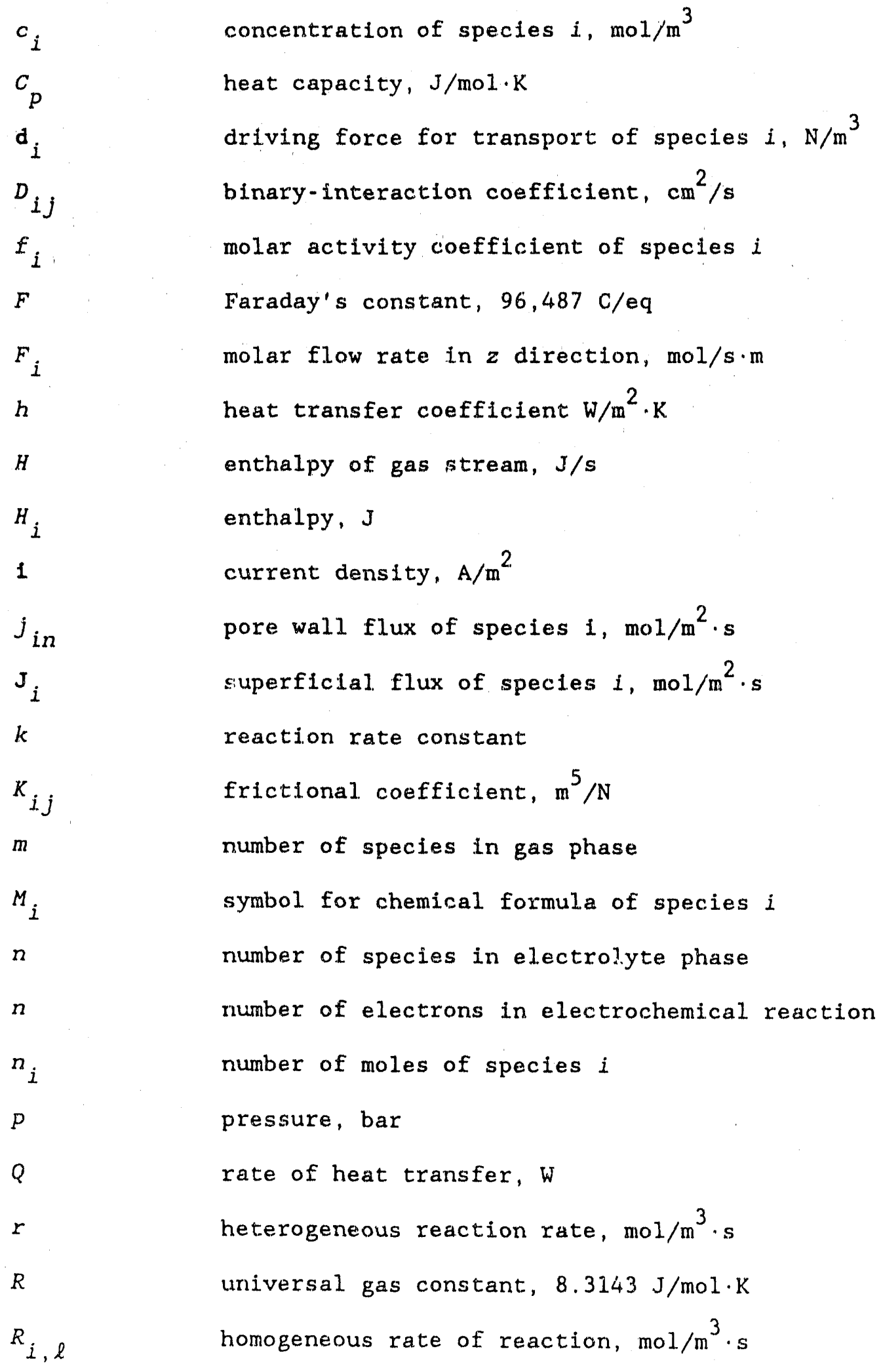




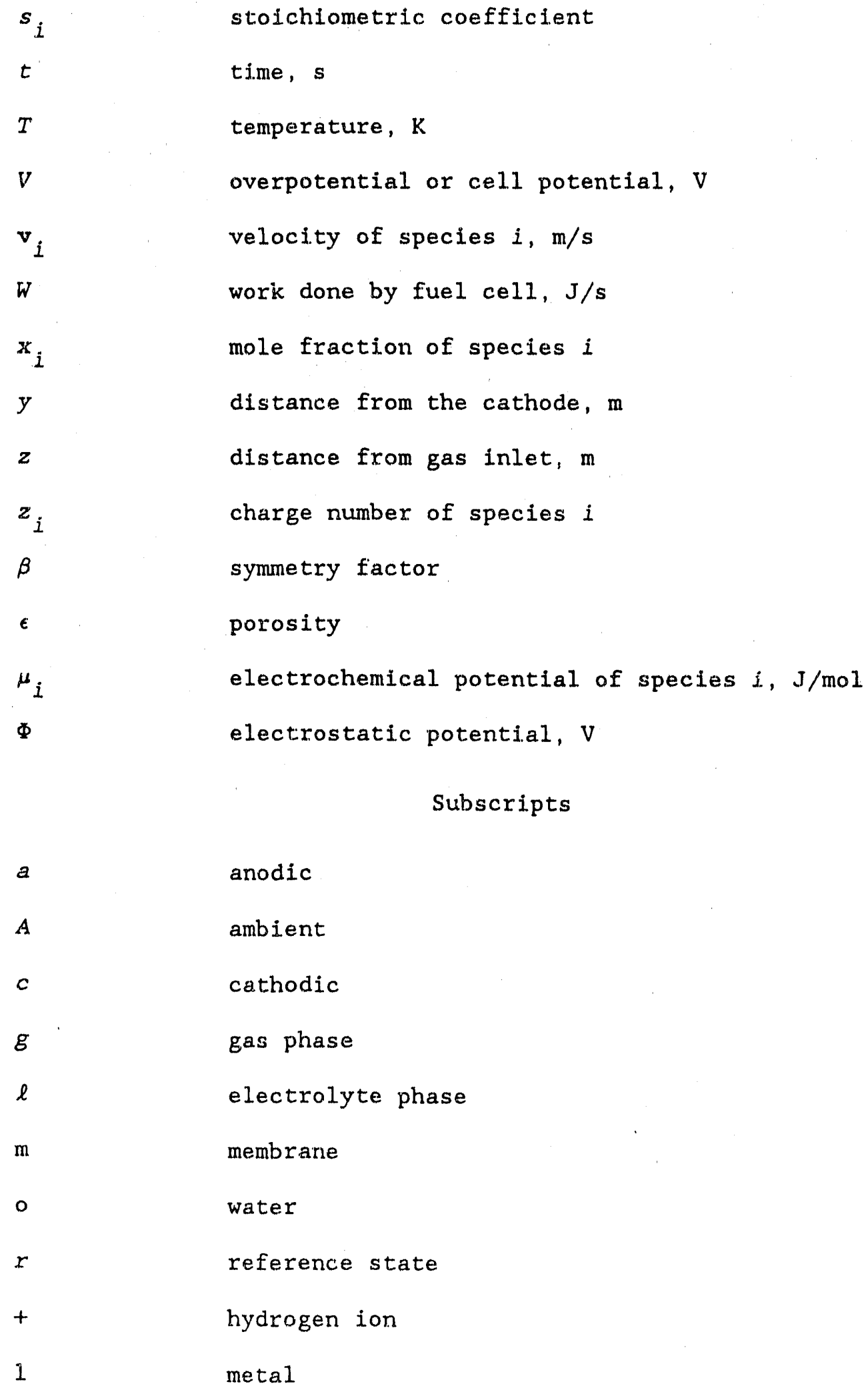


electrolyte

\begin{tabular}{ll} 
& \multicolumn{2}{c}{ Superscripts } \\
$-\quad$ & inlet condition or steady-state value \\
- & partial molar quantity \\
- & per mole \\
$*$ & low-pressure or ideal-gas linit
\end{tabular}

\section{References}

[1] E. A. Ticianel1i, C. R. Derouin, and S. Srinivasan, "Methods to Advance Technology of Proton Exchange Nembrane Fuel Cells," $J$. Electrochem. Soc., 135, 2209 (1988).

[2] J. Leddy and E. Vanderborgh, "Modeling of the Electrode/Ionomer Interface," in Diaphrams, Separators, and Ionexchange Membranes, edited by J. W. Van Zee, R. E. White, $\ddot{~}$. Kinoshita, and H. S. Burney, proceedings volume 86-13, The Electrochemical Society, Pennington, N. J. (1986).

[3] Fuel Cells, edited by W. Mitche11, Academic Press, New York (1963).

[4] J. Giner and C. Hunter, "The Mechanism and Operation of the Teflon-Bonded Gas Diffusion Electrode: A Mathematical. Mode1, " $J$. Electrochem. Soc., 116, 1124-1130 (1969).

[5] Handbook of Fuel Cell Technology, edited by C. Berger, Prentice Hall, Englewood Cliffs, N. J. (1968). 
[6] Michael C. Kimble and Ralph White, "A Mathematical Model of a Hydrogen/Oxygen Alkaline Fuel Cell," J. Electrochem. Soc, 138, $3370-3382$ (1991).

[7] Dawn M. Bernardi, "Water-Balance Calculations for SolidPolymer-Electrolyte Fuel Cells," J. Electrochen. Soc., 137, 3344-3350 (1990).

[8] Dawn M. Bernardi and Mark Verbrugge, Mathematical Model of Gas Diffusion Electrode Bonded to a Polymer Electrolyte," AIChE JournaI, 37, 1151-1.163 (1990).

[9] Dawn M. Bermardi and Mark Verbrugge, "Mathematical Model of the Soldd-Polymer-Electrolyte Fuel Ce1I," General Motors Research Laboratories, GMR-7360 (1991).

[10] T. E. Springer, T. A. Zawodzinski, and S. Gottesfeld, "Polymer Electrolyte Fuel Cell Model," J. Electrochem. Soc., 138, $2334-2342(1991)$.

[1.] R. Pollard and T. Comte, "Determination of Transport Properties for Solid. Electrolytes from the Impedance of Thin Layer Cells," $136,3734-3748$ (1989).

[12] John Newman, Electrochemical systems, 2nd edition, Prentlce-Hall, Englewood Cliffs, N. J. (1991).

(13) William H. Smyrl and John Newman, "Potentials of Cells with Liquid Junctions," J. Phys, Chem., 72, 4660 (1.968). 
[14] T. Zawodzinski, M. Neeman, L. Sillerud, and S. Gottesfeld, "Determination of Water Diffusion Coefficients in Perfluorosulfonated Ionomeric Membranes," J. Phys. Chem., 95, 6040-6044 (1991).

[15] W. Vogel, J Lundquist, P. Ross, and P. Stonehart, "The Rate Controlling step for klectrochemical Oxidation of Hydrogen on Pt in Acid and Poisoning of the Reaction by CO," Electrochim. Acta, 20, 79 (1975).

[16] D. Bernardi, E. Pawlikowski, and John Newman, "A General Energy Balance for Battery Systems," J. Electrochem. Soc., 132, 5-12 (1985).

(17) Bernard Tribollet and John Newman, "Impedance Model for a Concentrated Solution," J. Electrochem. Soc., 131, 2780-2785 (1984).

[1.8] D. Fan and R. E. White, "Modification of Newman's BAND(J) Subroutine to Multi-Region Systems Containing Interior Boundaries: MBAND," J. Electrochem. Soc., 138, 1688-1691 (1991). 


\section{Experimental Determination of the Transport Number of Water in Nafion 117 Membrane}

\subsection{Introduction}

A number of fuel-cell systems under development use perfluorinated ionomeric membranes for both the separator and the electrolyte. Proton conductivity is strongly coupled to the hydration of the polymer, and therefore it is vital to manage the water content in the separator of the solid-polymer-electrolyte fuel cell to maintain sufficient electrical conductivity for practical use. Cell efficiency is related to the transport properties of the membrane and may be the critical factor in load-leveling devices, for example, if the kinetics of the reactions are fast [1]. From the inception of solid polymer-electrolyte fuel cells, water-transport was recognized as a possible problem [2].

The motion of water is caused by a gradient in its chemical potential and by the movement of hydrogen ions, which is proportional to the current. The electroosmotic drag coefficient $\xi$,

$$
\xi=\frac{N_{0}}{N_{+}}
$$

is defined here as the number of water molecules moving with each hydrogen ion in the absence of concentration gradients. $\xi$ can be related to the transference and transport numbers of water, as will 
be shown later. Previous work shows that for each hydrogen ion that moves across a fully hydrated membxane about three water molecules are carried along [3] [4] [5].

Earlier methods to determine the electroosmotic drag coefficient measured the flux of water across the membrane at constant current. The membranes were always in direct contact with liquid water, and therefore, the water concentration in the membrane was fixed at $\lambda \approx 22$, where $\lambda$ is the number of water molecules per sulfonic acid group. Changes in the pretreatment of the membranes permit limited variation in the water content of the membranes that are equilibrated with liquid water. Springer et al. [6] used this approach to measure $\xi$ at $\lambda=11$. The pretreatment, however, can affect the txansport properties of the membrane as well. Furthermore, this method still does not allow examination of $\xi$ at low water contents, which is critical to the modeling of fuel-cell operation.

Water content in the membrane can be controlled over a wide composition range by equilibrating the membrane with a known partial pressure of water. Our attempts to wisure the flux of water from the gas phase and to eliminate concentration gradients were difficult and proved unreliable. An alternative approach using the open. circuit potentials of cells with transference was developed to meas ure the transport number of water in ionically conducting polymers over a large range of water contents. 


\subsection{Theory}

Transport properties can be obtained from electrochemical cells with a single electrolyte of varying concentration [7]. The cell potential depends not only on thermodynamics but also on the transport properties of the system in the transition region. The transport number of water in an ionically conducting polymer can be obtained by analyzing the concentration cell shown below.

$$
\begin{gathered}
\underset{\operatorname{Pt}(s), \mathrm{H}_{2}(g)}{\alpha} \underset{\operatorname{membrane}\left(\lambda_{\beta}\right)}{\beta}\left\{\begin{array}{c}
\text { transition } \\
\text { region }
\end{array}\right\} \underset{\text { membrane }\left(\lambda_{\gamma}\right)}{\gamma} \mid \operatorname{Pt}(s), \mathrm{H}_{2}(g) \\
\text { Concentration cell }
\end{gathered}
$$

The cell potential is

$$
F U=F\left(\Phi^{\delta}-\Phi^{\alpha}\right)=\left(\mu^{\alpha}{ }^{-}-\mu^{\delta}{ }^{-}\right)
$$

At the two electrodes, the electrochemical reaction

$$
\frac{1}{2} \mathrm{H}_{2} \rightleftarrows \mathrm{H}^{+}+\mathrm{e}^{-}
$$

is assumed to be in equilibrium, and therefore

$$
\frac{1}{2} \mu_{\mathrm{H}_{2}}=\mu_{+}+\mu_{\mathrm{e}}-
$$

The cell potential reduces to 


$$
F U=\frac{1}{2} R T \ln \frac{\mathrm{p}_{\mathrm{H}_{2}}^{\alpha}}{F_{\mathrm{H}_{2}}^{\delta}}+\int_{\mathrm{y}_{\beta}}^{\mathrm{y}_{\gamma}} \nabla \mu_{+} \mathrm{dy} .
$$

To analyze the system further we must examine the transport processes in the junction region. A gradient in chemical potential. of water exists in the transition region, and diffusion occurs. The multicomponent diffusion equation,

$$
c_{i} \nabla \mu_{i}-\sum_{j=i}^{n} K_{i j}\left(v_{j}-v_{i}\right),
$$

describes transport in the membrane. $c_{i} \nabla \mu_{i}$ is the driving force for transport, $v_{i}$ is the velocity of species $i$ relative to some arbitrary reference velocity, and $K_{i j}\left(-K_{j i}\right.$ from the onsager reciprocal relations) are the frictional coefficients. In general, for $r_{\text {s secies }}$ there are $k_{n}(n-1)$ independent transport properties necessary to characterize the system. The membrane is considered to be comprised of three components: water, hydrogen ions, and polymer, and therefore three independent transport properties are required. Because of the Gibbs-Duhem relationship and the fact that $k_{i j}-K_{j i}$, for $n$ species there are only $n-1$ independent equations of the form of equation 4. The membrane velocity is set to zero, and all fluxes are referenced to it. These $n-1$ equations, together with $N_{m}=0$, can be written in matxix form. With the electrochemical potential as the driving force, the multicomponent diffusion equations can be inverted to give 


$$
\mathbf{N}_{+}=c_{+} \mathbf{v}_{+}=-L_{++} c_{+}^{2} \nabla \mu_{+}-L_{+1} c_{+} c_{0} \nabla \mu_{0}
$$

and

$$
\mathrm{N}_{0}=c_{0} \mathbf{v}_{0}=-L_{0+} c_{0} c_{+} \nabla \mu_{+}-L_{00} c_{0}^{2} \nabla \mu_{0} .
$$

The $L_{i j}$ 's $\left(=L_{j i}\right)$ are related directly to the $K_{i j}$ :

$$
\begin{aligned}
& L_{o+}=\frac{K_{+o}}{K_{+o} K_{o m}+K_{+o} K_{+m}+K_{+m} K_{o m}}, \\
& L_{o o}=\frac{K_{+o}+K_{+m}^{K}}{K_{+o} K_{o m}+K_{+o} K_{+m}+K_{+m} K_{o m}},
\end{aligned}
$$

and

$$
L_{++}=\frac{K_{+o}+K_{o m}}{K_{+o} K_{o m}+K_{+o} K_{+m}+K_{+m} K_{o m}} .
$$

See Newman [8] (p. 276) for further details. Consequently, if experiments can be devised to measure the $L_{i j}$, then the binary interaction coefficients can be estimated as a function of water content within the membrane. This concept is the same as the orthogonal experiments described by Pintauro and Bennion [9]. For the Nafion membrane used in fuel-cell applications, the transport properties required are: the diffusion coefficient of water, the electrical conductivity, and the transport number of water. $L_{++}$is related to the electrical conductivity by 


$$
L_{++}=\frac{\kappa}{F^{2} c_{+}^{2}}
$$

From the definition of $\xi$, equations 5 and 6 give

$$
L_{o+}=\frac{\kappa \xi}{F^{2} c_{+} c_{0}} \text {. }
$$

For the concentration cell at open circuit depicted above, equation 5 can be written with the flux of hydrogen ions set to zero as

$$
\nabla \mu_{+}=-\xi \nabla \mu_{0} .
$$

Thus, if $\Delta \Phi$ represents the last term in equation 3 , then we can write

$$
F \Delta \Phi=F U-\frac{1}{2} R T \ln \frac{p_{H_{2}}^{\alpha}}{p_{H_{2}}^{\delta}}-\int_{y_{\gamma}}^{y_{\beta}} \xi \frac{d \mu_{o}}{d y} d y
$$

Everything in the integral is a function of water content, and therefore the equation can be integrated over the concentration. That is, the potential of the cell is independent of the shape of the concentration profile. Equation 13 becomes

$$
F \Delta \Phi=\int_{\lambda}^{\lambda} \xi \frac{d \mu_{O}}{d \lambda} d \lambda
$$

and differentiation gives 


$$
\xi=F \frac{d(\Delta \Phi)}{d \mu_{o}^{\beta}}
$$

This means that we would hold the water activity constant on the right side of the cell $(\gamma)$ and vary the activity of water on the left side $(\beta)$. Equation 15 then gives the value of $\xi$ at this water activity $(\beta)$.

An equivalent analysis can be made in terms of transference numbers. See, for example, Newman [8] (p. 279) for further information on the definitions and the details of this approach. The transference number is defined, in the absence of concentration gradients, by

$$
1 t_{i}^{m}-F z_{i} c_{i}\left(v_{i}-v_{m}\right)
$$

and is clearly related to the $L_{i j}$ 's or $K_{i j}$ 's of the multicomponent diffusion equations. The current expressed in terms of transference numbers is

$$
\frac{F}{\kappa} I=\sum_{i}\left(t_{i .}^{m} / z_{i}\right) \nabla \mu_{i}
$$

The ratio $t_{i}^{m} / z_{i}$, also called the transport number, is not necessarily zero for neutral species. For the Nafion system, all the current is carried by the protons, and consequently the transference number of the hydrogen ions is one. At open cixcuit, the current is zero, and 


$$
\frac{\nabla \mu_{+}}{z_{+}}=-\frac{t_{0}^{m}}{z_{0}} \nabla \mu_{0} .
$$

$\xi$ is defined in the absence of a gradient in the chemical potential of water and therefore is related to the transference number by

$$
\xi=\frac{\mathrm{N}_{0}}{\mathrm{~N}_{+}}-\frac{t_{0}^{m} z_{+}}{z_{0}} .
$$

Equation 12 results from combining equations 18 and 19 and setting $t_{+}^{m}=1$. Hereafter we shall use the ratio $t_{0}^{m} / z_{0}$ and $\xi$ interchangeably.

\subsection{Experimental}

Figure 1 shows a schematic of the concentration cell. A Nafion 117 (equivalent weight $=1100$, thickness dry $7 \mathrm{mils}-0.0178 \mathrm{~cm}$ ) membrane was placed between the two chambers, suspended above solutions of lithium chloride of different compositions. At each end of the membrane there was a platinum electrode. The electrodes and the membrane were in contact with hydrogen and water vapor at ambient pressure. Sufficient length from the barrier between the two chambers and the electrodes ensured that the activity of water in the membrane at each electrode was uniform and in equilibrium with the solution below it.

At equilibrium, the chemical potential of water is the same in the vapor phase and in the polymer electrolyte. Equation 15 becomes 


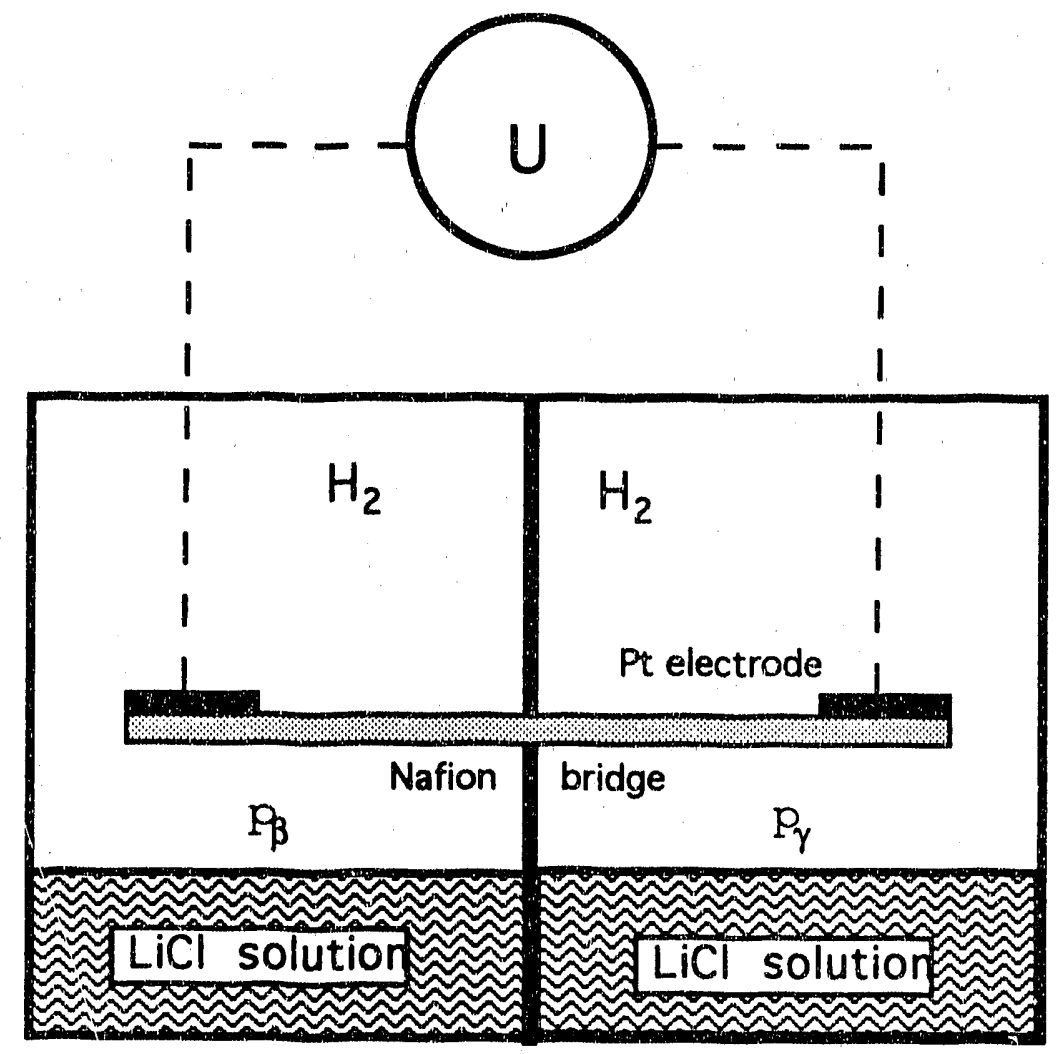

Figure 1. Schematic of concentration cell. The partial pressure of water is controlled with a lithium chloride solution. The LiCl concentration in the right side is maintained at $\mathrm{m}=0.1 \mathrm{~mol} / \mathrm{kg}$. 


$$
\xi\left(a_{0}^{\beta}\right)-\frac{F}{R T}\left(\frac{d\left(\Delta \Phi_{1}\right)}{d \ln p_{0}^{\beta} / p_{0}^{\gamma}}\right) .
$$

If the potential in the form $F \Delta \Phi / R T$ is plotted against the logarithm of the activity of water, the slope of the line is equal to $\xi$.

The partial pressure of water in each half of the cell was controlled using a lithium chloride solution. Robinson and stokes [10] and Gibbard and Scatchard [11] give the osmotic coefficient for a lithium chloride solution as a function of the concentration of the salt at various temperatures. The activity of water is calculated from

$$
\ln a_{0}=-\nu m M_{0} \phi .
$$

One side of the cell was filled with dilute lithium chloride solution, and the partial pressure of water remained constant for all experiments. A dilute solution was used in place of pure water to prevent the condensation of water on the membrane or on the sides of the cell. Lithium chloride solutions of various concentrations were put in the other side. To minimize evaporation from the cell, hydrogen gas passed through a gas-washing bottle filled with a solution of identical composition to that in the corresponding chamber before entering the cell.

The membranes were pretreated with $1 \mathrm{M}$ boiling sulfuric acid to convert to the hydrogen form, and thoroughly rinsed in deionized water. The electrodes were fabricated from platinum mesh that was 
platinized with Hellige platinizing solution to ensure highly reversible reactions. Nevertheless, experiments with nonplatinized electrodes gave identical results. The mesh was spot welded to platinum wire, which served as the electrical lead. The entire cell was placed in a water bath maintained at a constant temperature $\pm 0.1^{\circ} \mathrm{C}$.

After the addition of a new solution of lithium chloride, the system was purged with nitrogen to remove oxygen from the cell, and then an equal flow of hydrogen was established to each side. The system was allowed to reach a quasi steady state; a slow relaxation of the cell occurs because of the gradient in chemical potential of water across the cell. Steady state was attained in a few hours; the transient was most likely associated with the space-time for the hydrogen gas. The cell potential was measured with a Hewlett Packard inodel 3456A digital voltmeter, with an input resistance of greater than $10 \mathrm{M} \Omega$. This was checked against a Keithly electrometer model 602 with an input impedance of greater than $14 \mathrm{M} \Omega$ with identical results. The potential was measured over a period of about two days, with sampling about every hour.

$\Delta \Phi$ is calculated from equation 13 . The potential due to differences in hydrogen pressure, which is important at higher temperatures (see equation 3), was calculated assuming a total pressure of 1.01 bar and subtracting the partial pressure of water. The errors because of slight differences in the total pressure in the two chambers was believed to be small, but was not measured. 


\subsection{Results and Discussion}

Figure 2 shows the measured potentials of the cell. Each line corresponds to a set of experiments with a different membrane at a given temperature. The error bars for run 4 represent the range of the measured potentials. The results are also summarized in table 1 . Along with the mean value, we report the range of measured potentials.

Table 1.

Run \#1 $25^{\circ} \mathrm{C}$

\begin{tabular}{|c|c|c|c|}
\hline$\frac{P_{\beta}}{P_{\gamma}}$ & \multicolumn{1}{|c|}{$\frac{F U}{R T}$} & + & - \\
\hline 1.000 & -0.000 & 0.0 & 0.0 \\
0.967 & -0.007 & 0.006 & 0.003 \\
0.873 & -0.176 & 0.028 & 0.039 \\
0.681 & -0.468 & 0.058 & 0.059 \\
$0.1 \% 13$ & -1.038 & 0.064 & 0.104 \\
0.201 & -1.816 & 0.015 & 0.219 \\
\hline
\end{tabular}

The relayation of the cell is thought to cause the variations in potential. Since a concentration gradient exists, water is transRun $\# 225^{\circ} \mathrm{C}$

\begin{tabular}{|c|c|c|c|}
\hline$\frac{P_{\beta}}{P_{\gamma}}$ & \multicolumn{1}{|c|}{$\frac{F_{R U}}{R T}$} & + & - \\
\hline 1.000 & -0.000 & 0.0 & 0.0 \\
0.967 & -0.110 & 0.032 & 0.041 \\
0.814 & -0.386 & 0.042 & 0.055 \\
0.681 & -0.642 & 0.049 & 0.026 \\
0.413 & -1.1 .41 & 0.011 & 0.067 \\
0.201 & -1.874 & 0.024 & 0.043 \\
\hline
\end{tabular}




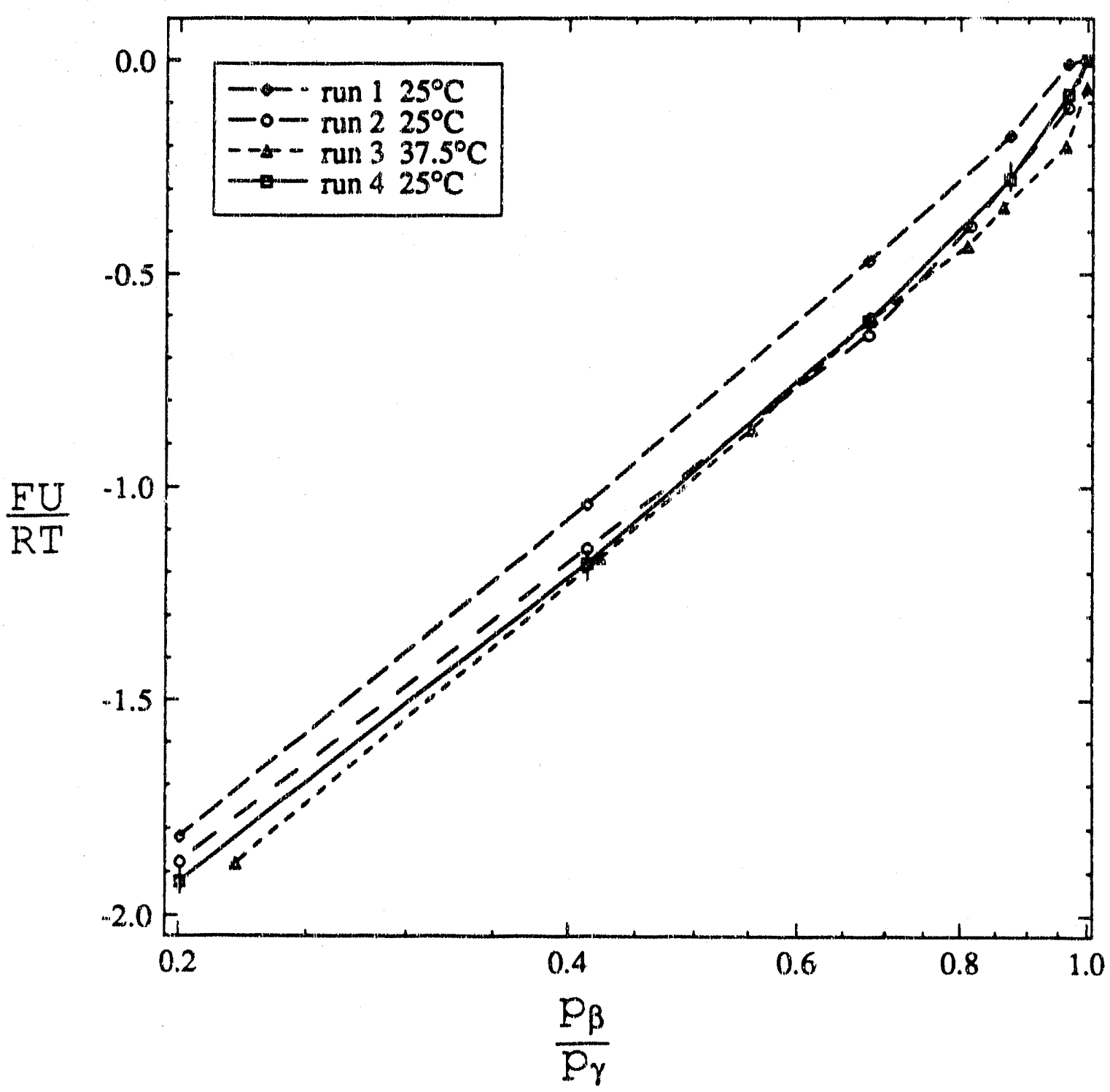

Figure 2. Measured cell potential. Each line represents a set of data with a different membrane. Data points are averaged over two days, sampling about every hour. Error bars are included for run 4 and represent the range of measured potentials. 
Run $37.5^{\circ} \mathrm{C}$

\begin{tabular}{|c|c|c|c|}
\hline$\frac{p_{\beta}}{p_{\gamma}}$ & $\frac{F U}{R T}$ & + & - \\
\hline 0.999 & -0.065 & 0.080 & 0.044 \\
0.963 & -0.200 & 0.020 & 0.050 \\
0.863 & -0.342 & 0.066 & 0.110 \\
0.809 & -0.434 & 0.032 & 0.045 \\
0.684 & -0.606 & 0.022 & 0.027 \\
0.553 & -0.864 & 0.014 & 0.020 \\
0.423 & -1.164 & 0.029 & 0.064 \\
0.221 & -1.878 & 0.027 & 0.067 \\
\hline
\end{tabular}

Run $\# 24^{\circ} \mathrm{C}$

\begin{tabular}{|c|c|c|c|}
\hline$\frac{p_{\beta}}{p_{\gamma}}$ & $\frac{F U}{R T}$ & + & - \\
\hline 1.000 & -0.000 & 0.0 & 0.0 \\
0.967 & -0.080 & 0.008 & 0.006 \\
0.873 & -0.276 & 0.037 & 0.027 \\
0.681 & -0.610 & 0.015 & 0.027 \\
0.413 & -1.176 & 0.028 & 0.040 \\
0.201 & -1.920 & 0.027 & 0.028 \\
\hline
\end{tabular}

ported across the membrane. The variations are larger than predicted and suggest that a perfect seal was not obtained. Nevertheless, this resulted in only a slow reduction in the magnitude of the cell potential with time.

The data were fitted with an analytic expression (as shown in figure 3), and that expression was differentiated rather than differentiating the data directly. Many functional forms were tried to fit the data, using a least-squares routine to minimize the total error. In order not to prejudice the results, we only required that the potential approach a constant as the activity of water approaches 


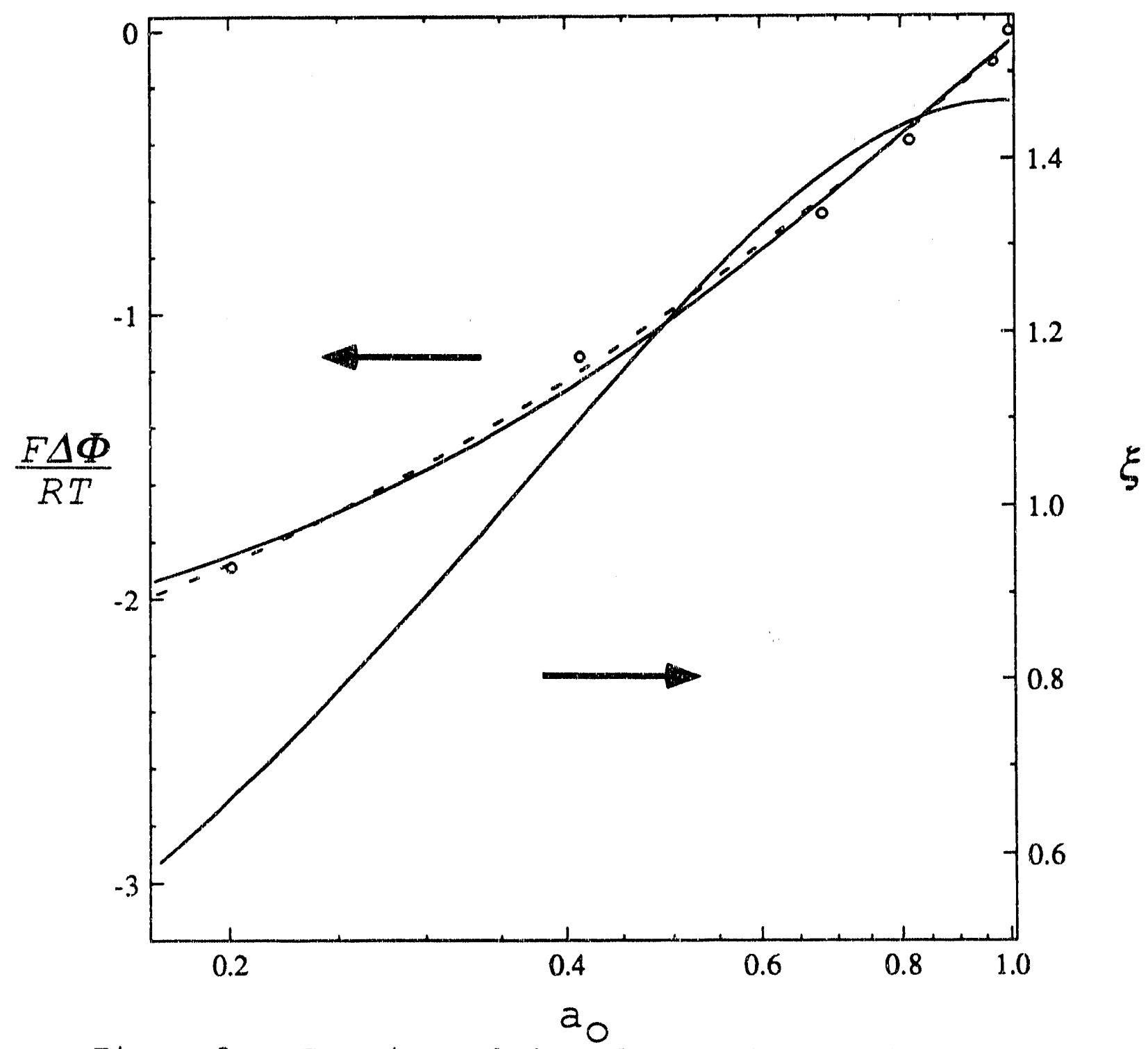

Figure 3. Experimental data for run 2, the fitted curves using equation 22 (solid line) and a second order polynomial (dashed line), and the transport number of water from equation 23. 
zero. A three-parameter polynomial and an exponential function gave the best results. The computer program is listed in the appendix. The latter form used to interpret the data was

$$
\frac{F \Delta \Phi}{R T}=A+B \exp \left(-C a_{0}\right)
$$

The transport number of water, therefore, is

$$
\frac{t_{0}^{m}}{z_{0}}=-B C a_{0} \exp \left(-C a_{0}\right)
$$

The results are summarized in table 2 . Leaks in the cell prevented making measurements at higher temperatures, although the transport number of water is not expected to vary dramatically with small changes in temperature. The entire cell was immersed in a water bath; and at higher temperatures, water seeped into the cell. In the future, the cell should be placed in a corvection oven, as is done in many fuel-cell experiments, to eliminate this problem. Runs 2 and 4 gave the most reproducible results. Improvements in the experimental technique are believed to have caused the differences between these two and the first run. The fit of data from xun 2 is shown in figure Table 2 .

\begin{tabular}{|c|c|c|c|c|}
\hline run & ${ }^{\circ} \mathrm{C}$ & $\mathrm{A}$ & $\mathrm{B}$ & $\mathrm{C}$ \\
\hline 1 & 25.0 & 0.6165 & -3.4377 & 1.717 \\
2 & 25.0 & 1.3918 & -3.9851 & 1.017 \\
3 & 37.5 & 0.6994 & -3.5519 & 1.454 \\
4 & 25.0 & 1.1777 & -3.9078 & 1.167 \\
\hline
\end{tabular}


3 for both a polynomial and an exponential fit. Since this is a derivative method, the greatest error is expected at the ends. As the concentration of water tends to zero, the transport number must also approach zero, which implies that the potential must become constant; but there is no similar asymptote as the membrane becomes fully hydrated.

To relate the transport number of water to the concentration of water in the membrane, instead of the activity of water, an absorption isotherm is needed. This thermodynamic relationship was measured at $30^{\circ} \mathrm{C}$ by Zawodzinski et al.[6] [12]. In the absence of other data, this relationship was assumed to hold at other temperatures; that is, $\lambda$ at $a_{0} m-1$ is independent of temperature. Figure 4 shows the results at $25^{\circ} \mathrm{C}$ of the present analysis; the theoretical maximum value, which is discussed below, and other data available in the literature.

At $25^{\circ} \mathrm{C}$, the electroosmotic drag coefficient is about 1.4 , decreases with lower water content at moderate hydration, and falls sharply as the membrane is fully dehydrated. The values are not in complete agreement with those measured at $\lambda=11$ [6]. The differences in pretreatment could cause the discrepancy. At low water contents, the value of $\xi$ begins to drop sharply in accordance with the theoretical maximum. Physically we can picture that the first waters of hydration are tightly bound, and therefore the transport number is close to one. Only when the membrane is dehydrated does the transport number fall below one and approach zero. A membrane that has 


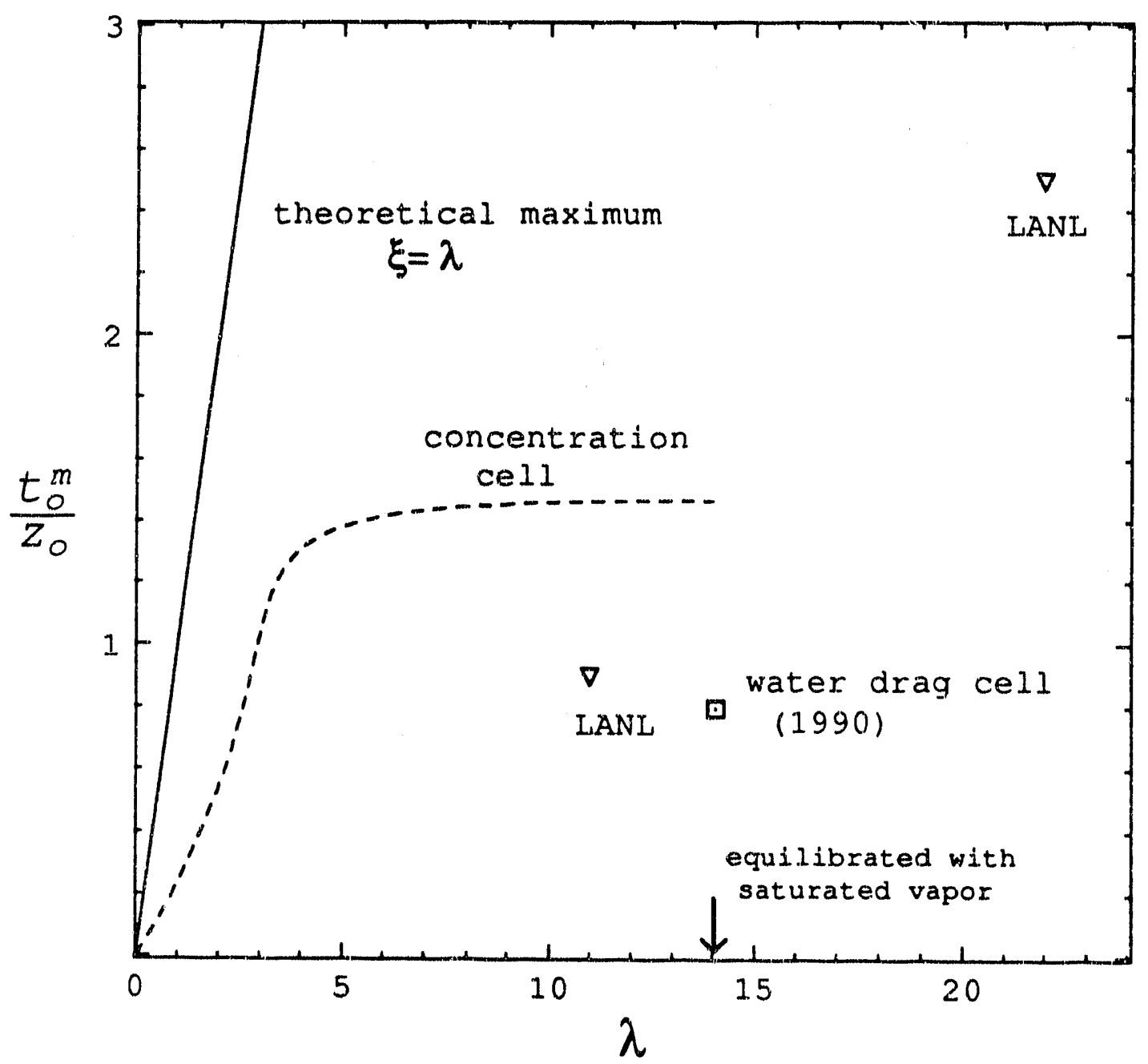

Figure 4. Transport number of water as a function of the concentration of water at $25^{\circ} \mathrm{C}$. Dashed line represents the results of the present work based on data for 1 between 14 and 2. Triangles are values measured at Los Alamos National Laboratory [6], and the square is our unpublished result from the gas-phase flux measurements. 
received identical pretreatment can have a different water content when exposed to liquid water as opposed to saturated water vapor [13]. Although not affecting our work, this anomaly is apparent when one compares our results to those measured in liquid water, as figure 4 shows.

The coupling between transport processes precludes one from obtaining any coefficient completely independently of the others. For an isothermal, isobaric system, with no chemical reactions, the equation of entropy production is given by

$$
-\frac{1}{T} \sum_{i} v_{i} \cdot d_{i},
$$

which must be positive. Any experimental determination of the transport properties should be consistent with the second law of thermodynamics. Equation 4 is substituted into 24, and for our ternary system

$$
\left(K_{+o}+K_{o m}\right) v_{o}^{2}-2 K_{o+} v_{o} v_{+}+\left(K_{o+}+K_{+m}\right) v_{+}^{2}>0
$$

Therefore, to ensure positive production of entropy

$$
\begin{aligned}
& K_{\text {otn }}+K_{o+}>0, \\
& K_{m+}+K_{o+}>0
\end{aligned}
$$

and 


$$
K_{o+}^{2}<\left(K_{o m}+K_{o+}\right)\left(K_{m+}+K_{o+}\right) .
$$

Finally we can conclude that

$$
L_{++}>0
$$

and

$$
L_{\infty O}>0
$$

These last two equations are equivalent to the requirement of the second law of thermodynamics that the conductivity and diffusivity be positive quantities.

The second law, however, makes no restriction on $L_{o+}$, or on the transport number of water. Although it is physically reasonable for the frictional coefficient to be positive, $K_{i j}$ may be less than zero without violating the second law of thermodynamics. Robinson and Stokes [10] for example, discuss cation transference numbers that are negative for zinc halide systems. In these systems, the zinc is believed to be complexed with the halides to form a negative ion, which results in a negative transference number for the zinc cation. Thus, we can still argue that if the speciation is done properly we will not obtain negative transport numbers. If the speciation is correct for our system and the frictional coefficients are positive, one can conclude from equations 7 to 11 that

$$
0<\xi<\lambda
$$


and

$$
D_{0}>\frac{\kappa \xi}{F^{2}}(\lambda-\xi) \frac{d \mu_{0}}{d c_{0}} \text {. }
$$

Equation 28 ensures that $K_{o m}$ is positive. A larger value of $\xi$ would indicate that the interactions between water and the membrane were negative. The same result may be obtained fxom simple physical arguments. From the definition of $\xi$,

$$
\xi=\frac{N_{0}}{N_{+}}=\frac{c_{0}^{v} 0}{c_{+}{ }^{v}}
$$

for a small current density passed through the membrane and a uniform chemical potential of water, equation 28 means that $\mathrm{v}_{0}<\mathrm{v}_{+}$. Violation of the inequality of equation 28 would imply that the membrane was accelerating the water rather than retarding it. The solid line in figure $4, \xi-\lambda$, is the maximun value from the inequality of equation 28. Analyzing equation 29 requires data for the conductivity and diffusion coefficient of water and is not discussed further here.

The dependence of the transport number of water on concentration is needed in the modeling of fuel cells. Although in our experiments transport was perpendicular to the direction of current flow in a fuel cell, we believe the membrane is isotropic. This is supported by the recent work of Verbrugge and Hill [14]. Two assumptions that have been used are: the transport number is independent of composition [15] or $\xi$ is proportional to $\lambda[6]$. With these assumptions, we 
can integrate equation 14 and compare the predicted potential of the cell to the experimental measurements, as figure 5 shows. The assumption that the transport number is proportional to $\lambda$ predicts a much lower potential than was observed and is clearly not consistent with the experimental data at low water contents. Assuming the transport number of water is constant is also inconsistent with the data as well as the theoretical maximum derived earlier. The data show that $\xi$ must increase with the concentration of water. The results of the present analysis combine the best features of the two assumptions given above.

\section{List of Symbols}

$\begin{array}{ll}a_{0} & \text { activity of water } \\ A & \text { constant in equation } 22 \\ B & \text { constant in equation } 22 \\ C_{i} & \text { concentration of species } i, \mathrm{~mol} / 1 \\ C & \text { constant in equation } 22 \\ \mathrm{~d}_{i} & \text { driving force for transport of species } i, \mathrm{~N} / \mathrm{m}^{3} \\ D_{0} & \text { mutual diffusion coefficient of water, } \mathrm{cm}^{2} / \mathrm{s} \\ F & \text { Faraday's constant, } 96,487 \mathrm{C} / \mathrm{eq} \\ F & \text { current density, A/cm } \\ K_{i j} & \text { frictional coefficient defined by equation }(1), \mathrm{J} \cdot \mathrm{s} / \mathrm{m}^{5} \\ L_{i j} & \text { coefficient defined in equations }(4 \text { to } 6), \mathrm{m}^{5} / \mathrm{J} \cdot \mathrm{s} \\ m & \text { molality, mol/kg }\end{array}$




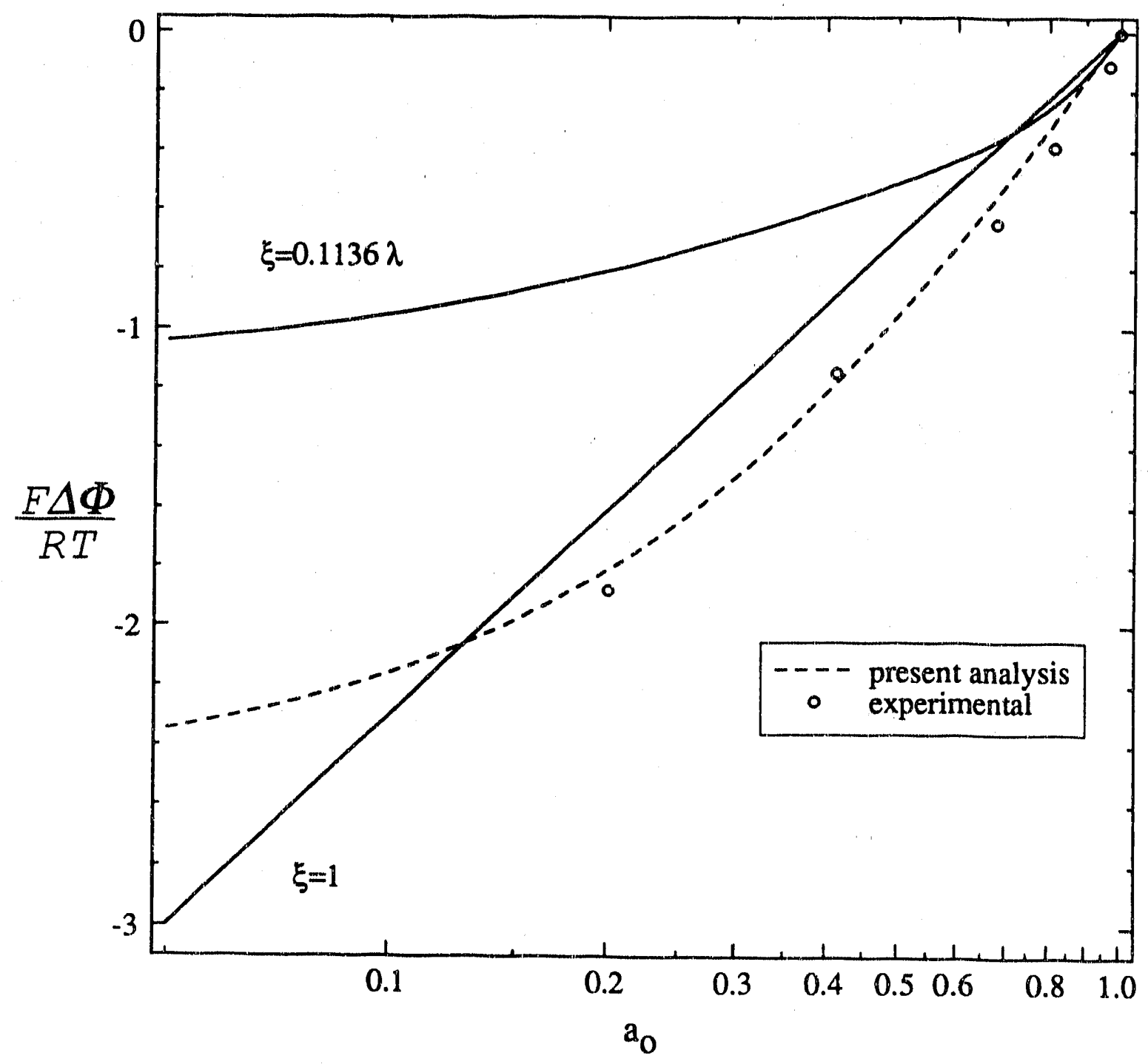

Figure 5. Comparison of the measured potential of the cell with predictions from two assumptions for variation of the transport number with concentration, as well as the present analysis. 


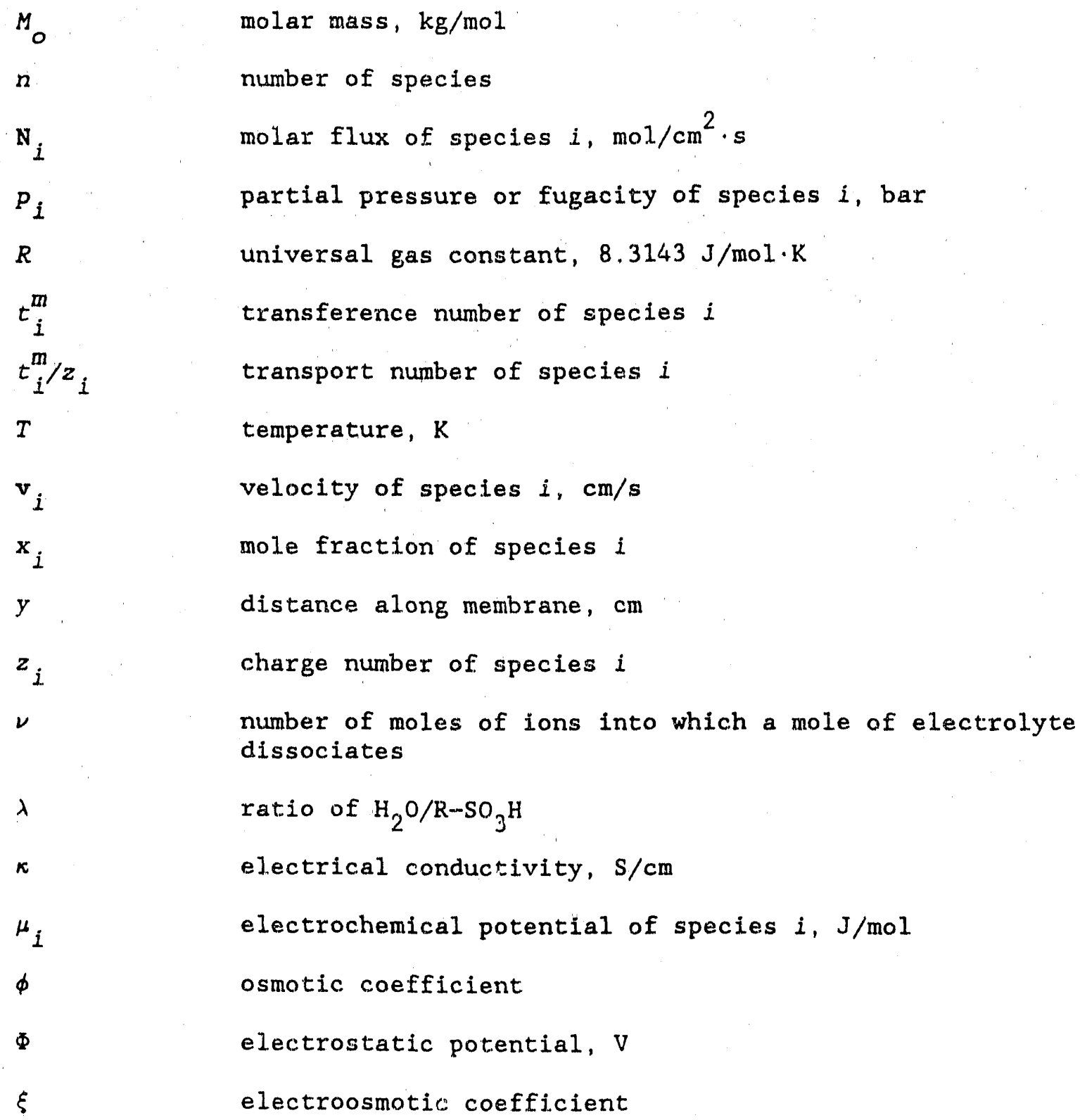

Subscripts

$\begin{array}{ll}\text { g } & \text { gas } \\ \text { in } & \text { membrane } \\ \text { o } & \text { water }\end{array}$




$$
\begin{array}{ll}
s & \text { solid } \\
+ & \text { hydrogen ion }
\end{array}
$$

\section{References}

[1] R. S. Yeo, "Applications of Perfluorosulfonated Polymer Membranes in Fuel Ce11, Electrolyzers, and Load Leveling revices," in Perfluorinated Ionomer Membranes, edited by A. Eisenberg and H. L. Yeager, Amer. Chem. Soc. Symposium Series No. 180, Washington, D.C. (1982).

[2] Carl Berger, ed., Handbook of Fuel Cell Technology, Prentice-Hall, Inc., Englewood Cliffs, N. J. (1968).

[3] J. L. Fales, N. E. Vanderborgh, and P. Stroeve, "The Influence of Ionomer Channel Geometry on Ionic Transport," in Diaphragms, Separators, and Ion-Exchange Membranes, edited by J. W. Van Zee, R. E. White, K. Kinoshita, and H. S. Burney, PV 86-13, p. 179, The Electrochemical Society Softbound Proceedings Series, Pennington, N. J. (1986).

[4] A. B. LaConti, A. R. Fragala, and J. R. Boyack, in Proceedings of the Symposium on Electrode Materials and Processes for Energy Conversion and Storage, edited by J. D. E. McIntyre, S. Srinivasan, and F. G. Will, The Electrochemical Society, Inc., Pennington, N. J. (1977).

[5] Los Alamos National Laboratory Report, Second quarterly progress report (1989). 
[6] T. E. Springer, T. A. Zawodzinski, and S. Gottesfeld, "Polymer Electrolyte Fuel Cell Model," J. Electrochem. Soc., 138, 2334 $2342(1991)$.

[7] W. Smyrl and J. Newman, "Potentials of Cells with Liquid Junctions," J. Phys. Chem., 72, 298-303 (1968).

[8] John Newman, Electrochemical Systems, 2nd edition, Prentice-Ha 11 Inc., Englewood Cliffs, N.J. (1991).

[9] P. N. Pintauro and D. N. Bennion, "Mass Transfer of Electrolytes in Membranes. 1 . Development of Mathematical Model and 2. Determination of $\mathrm{NaCl}$ Equilibrium and Transport Parameters for Nafion," Ind. Eng. Chem. Fund., 23, 230-243 (1.984).

[10] R. A. Robinson and R. H. Stokes, Electrolyte Solutions, Butterworths, London (1968).

[11] H. F. Gibbard, Jr., and G. Scatchard, "Liquid-Vapor Equilibrium of Aqueous Lithium Chloride, from 25 to $100^{\circ} \mathrm{C}$ and from 1.0 to 18.5 Molal, and Related Properties," J. Chem. Eng. Data, 18, 293-298 (1973).

[12] T. Zawodzinski, M. Neeman, L. Sillerud, and S. Gottesfeld, "Determination of Water Diffusion Coefficients in Perflucrosulfonated Ionomeric Membranes," J. Phys. Chem., 95, 6040-6044 (1991).

[13] T. A. Zawodzinski, F. Garzon, V. T. Smith, and S. Gottesfeld, "Thermodynamic and Kinetic Studies of Water in Perfluorinated 
Ionomers," Extended Abstracts of the 178 th Society Meeting (The Electrochemical Society, Fall 1990), Abstract No. 117.

[14] M. W. Verbrugge and R. F. Hill, "Measurement of Ionic Concentration Profiles in Membranes During Transport," Electrochimica Acta, 37, 221-229 (i992).

[15] T. F. Fuller and J. Newman, "A Concentrated Solution Theory Model of Transport in Solid-Polymer-Electrolyte Fuel Cells," Proceeding of the Symposium on Fuel Cells, edited by R. E. White and A. J. Appleby, proceedings volume 89-14, The Electroshemical Society, Penningtion, N. J. (1989). 
Append1x A

c program for fitting data of open-circutt vs a0.

impliclt real*8(a-h,o-z)

dimension $x(20), y(20), b(6,6), d(6,1)$

common $b, d$

common / fn/ aa

read *, AA

read $*$, ndata

read $*,(x(i), y(i), i=1$, ndata $)$

$n=1$

do $11-1, n$

do $1 \mathrm{k}=1, \mathrm{n}$

$b(k, 1)=0.0 \mathrm{~d} 0$

do 1 1-1, ndata

$1 b(k, 1)-b(k, 1)+f(k, x(i)) * f(1, x(1))$

print *, $b(1,1)$

do $2 \mathrm{~km}, \mathrm{n}$

$\mathrm{d}(\mathrm{k}, 1)=0.0 \mathrm{~d} 0$

do 2 i -1 , ndata

$2 d(k, 1)=d(k, 1)+f(k, x(i)) * y(i)$

print *, $d(1,1)$

call matinv $(1, n$, determ)

print *, ' determ', determ

print *, $(d(k, 1), k=1, n)$

101 format (' $\left.d\left({ }^{\prime}, 12,{ }^{\prime}\right)-', e 15.5\right)$

eps -0.0 do

do 4 i-1, ndata

$\mathrm{y}^{\mathrm{c}} \mathrm{c}-0.0$

do $3 \mathrm{k}-1, \mathrm{n}$

$3 y c-y c+d(k, j) * f(k, x(1))$

print 103, yc, $y(i), x(i)$

103 format ( $3 f 12.5$ )

4 eps-epst $(y(i)-y c) * * 2$

c

print 102, aa, eps

print *, aa, eps

102 format ('aa-', f10.4,' eps=',f10.4)

end

function $f(k, x)$

IMPLICIT REAL*B(A-H,O-Z)

common / $\mathrm{fn} / \mathrm{aa}$

$f=1.0-\left(1.0-\operatorname{dexp}\left(-a a^{*} x\right)\right) /(1.0-\operatorname{dexp}(-a a))$

return

end

SUBROUTINE MATINV (N, M, DETERM)

IMPLICIT REAL*8 $(A-H, O-Z)$

COMMON $B(6,6), D(6,1)$

DIMENSION ID $(6)$

DETERM $=1.0$

DO $\& I-1, N$ 
1 ID (I) $=0$

DO $18 \mathrm{NN}=1, \mathrm{~N}$

$\mathrm{BMAX}=1.1$

DO $6 \quad I=1, N$

IF(ID(I).NE.0) GO TO 6

BNEXT $=0.0$

BTRY $=0.0$

DO $5 \mathrm{~J}=1, \mathrm{~N}$

$\operatorname{IF}(I D(J) . N E .0)$ GO TO 5

$\operatorname{IF}(\operatorname{ABS}(B(I, J))$ ).LE. BNEXT) GO TO 5

$\operatorname{BNEXT}-A B S(B(I, J))$

IF (BNEXT.LE.BTRY) GO TO 5

BNEXT-BTRY

$B T R Y=A B S(B(I, J))$

$\mathrm{JC}=\mathrm{J}$

5 CONTINUE

IF (BNEXT.GE. BMAX*BTRY) GO TO 6

BMAX-BNEXT/BTRY

IROW $=$ I

JCOL $=\mathrm{JC}$

6 CONTYNUE

IF (ID (JC).EQ.0) GO TO 8

DETERM $=0.0$

RETURN

$8 \operatorname{ID}(\mathrm{JCOL})=1$

IF (JCOL.EQ.IROW) GO TO 12

DO $10 \mathrm{~J}=1, \mathrm{~N}$

SAVE $=B$ (IROW , J)

$B$ (IROW, J) $-B(J C O L, J)$

$10 \quad B(J C O L, J)-S A V E$

DO $11 \mathrm{~K}=1, \mathrm{M}$

SAVE=D (IROW, K)

$D(I R O W, K)=D(J C O L, K)$

$11 \mathrm{D}(\mathrm{JCOL}, \mathrm{K})=\mathrm{SAVE}$

$12 \quad \mathrm{~F}=1.0 / \mathrm{B}(\mathrm{JCOL}, \mathrm{JCOL})$

DO $13 \mathrm{~J}=1, \mathrm{~N}$

$13 B(J C O L, J)=B(J C O L, J) * F$

DO $14 \mathrm{~K}=1, \mathrm{M}$

$14 D(J C O L, K)=D(J C C T, K) * F$

DO $18 \quad I=I, N$

IF (I.EQ.JCOL) GO TO 18

$F-B(I, J C O L)$

DO $16 \mathrm{~J}=1, \mathrm{~N}$

$16 B(I, J)-B(I, J)-F * B(J C O L, J)$

DO $17 \mathrm{~K}=1, \mathrm{M}$

$17 D(I, K)-D(I, K)-F * D(J C O L, K)$

18 CONTINUE

RETURN

END 


\section{Diffusion Coefflclent of Water and Calculation of Binary-interaction Coefficlents}

\subsection{Introduction}

One of the three transport properties required to characterize transport in the membrane of a solid-polymer-electrolyte fuel cell is the diffusion coefficient of water. We were concerned with two issues. First, the diffusion coefficient must be well-defined and suitable for our model. Second, to evaluate water management in the separator of the fuel cell, we need the diffusivity as a function of the level of hydration of the membrane.

There have been many measurements of diffusion coefficients in Nafion membrane reported in the literature [1] [2] [3] [4] [5] [6] [7] [8]. In the majority of experiments, however, only cation diffusion coefficients are reported, or the membranes were in contact with a liquid electrolyte. Yeo and Eisenberg [9] used a transient tech. nique and found the diffusion coefficient of water to vary with temperature as

$$
D_{0}=6.0 \times 10^{-3} \exp \left(\frac{-2436}{T}\right)
$$

The work of Zawodzinski et al. [10] was the most significant for the solid-polymer-electrolyte fuel cell. They used pulsed field gradient spin echo NMR measurements to determine the intra-diffusion 
coefficient of water in a Nafion membrane. They reported the diffusivity of water at different levels of hydration, $0.6 \times 10^{-6} \mathrm{~cm}^{2} / \mathrm{s}$ at $\lambda=2$ to $5.8 \times 10^{-6} \mathrm{~cm}^{2} / \mathrm{s}$ at $\lambda=14$.

Robinson and Stokes [11] and Crank [12] review the definitions and methods of measuring diffusion coefficients. Three standard steady-state techniques are the diaphragm cell, conductometric method, and optical methods. Measurements using the diaphragm cell, with water vapor on each side of the membrane, are impractical for the polymer electrolyte, because the amount of water contained in the membrane is large compared to the amount in the humidified gases. The conductometric method is discussed in a later section. Optical methods may be possible, but were not investigated here.

An intra-diffusion coefficient can be measured, using radiotracer techniques or NMR, for example. It is difficult, nonetheless, to relate these directly to the binary-interaction parameters of interest. This will be discussed later in this chapter.

For a binary systein, one form of Fick's law is [13]

$$
\mathrm{n}_{0}=-\rho D_{0} \nabla_{0}+\rho \omega_{0} \mathrm{v}
$$

Here, the membrane is stationary; therefore, the molar flux can be written as

$$
\mathrm{N}_{0}=\frac{-\rho D_{0}}{\left(1-\omega_{0}\right) M \omega_{0}} \nabla \omega_{0}=-\alpha \nabla \mu_{0}
$$


The coefficient $\alpha$ and $D_{0}$, the mutual diffusion coefficient, are related by

$$
\alpha=\frac{\rho D_{0}}{\left(1-\omega_{0}\right) M W_{0}} \frac{d \omega_{0}}{d \mu_{0}} \text {. }
$$

The above equations provide a well-defined diffusion coefficient that is suitable for modeling. We did not find an experimental method of measuring the diffusion coefficient of water that satisfied our objectives. The only measurements of diffusion coefficients as a function of level of hydration were those in reference [11]. It is not clear how to relate these NMR results to the $D_{0}$ of equation 1. Consequently, we wanted to have an estimate of the diffusion coefficlent, based on the well-defined equations given above, to compare wi.th the intra-diffusion coefficients of [11].

\subsection{Measurement of the Diffusion Coefficient}

A simple experiment was devised to determine the magnitude of the diffusion coefficient. In the diffusion cell shown in figure 1 , one side of the membrane is equilibrated with liguid water. The concentration in the membrane at this interface is determined through an equilibrium relationship, $\lambda-18$. On the other side of the cell, dry nitrogen impinges on the surface of the membrane.

Figure 2 shows the experimental apparatus. Water that is transported through the membrane is measured by collecting in a desiccant tube containing calcium sulfate. One calculates the flux of water 


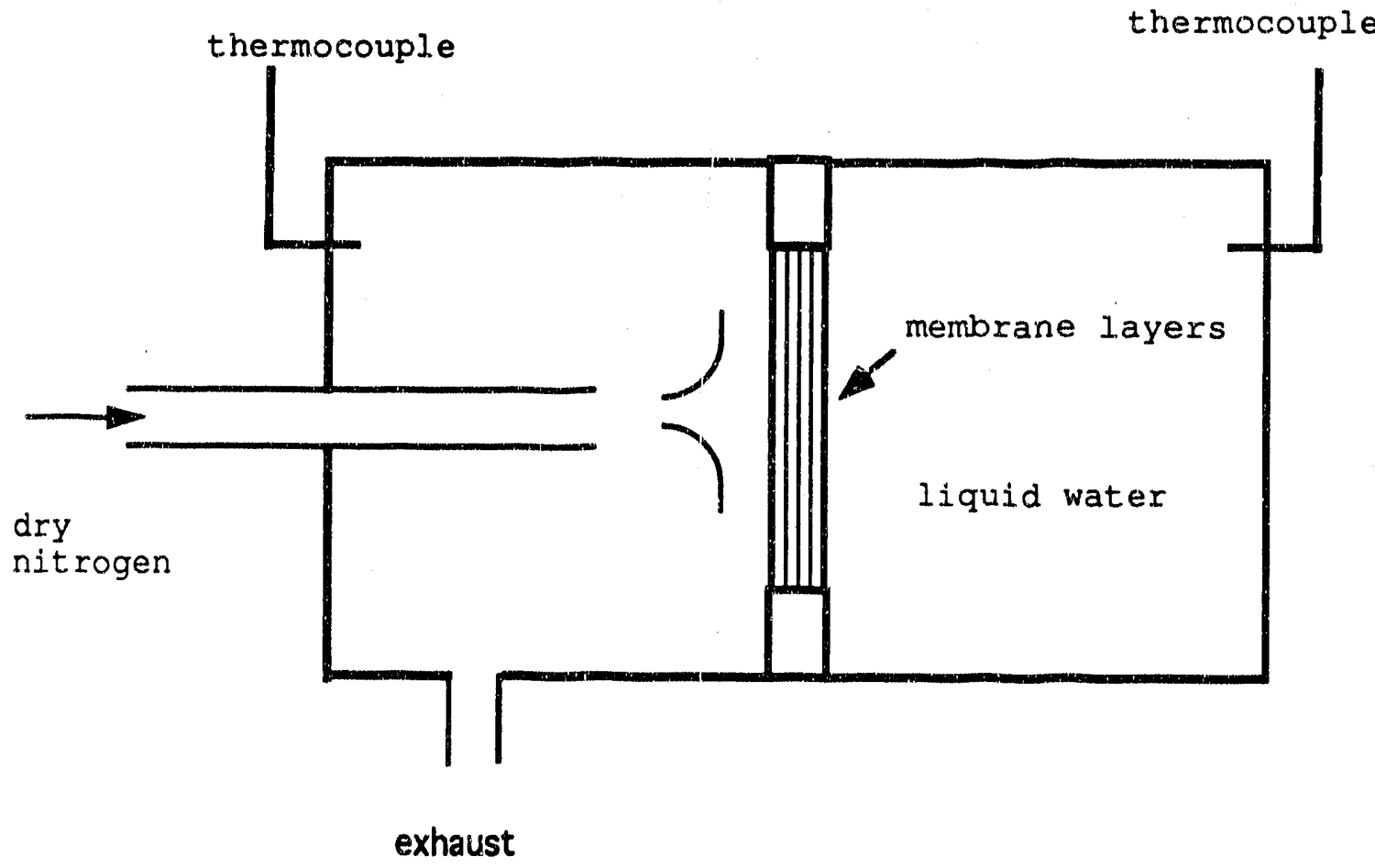

Figure 5. Diffusion cell 


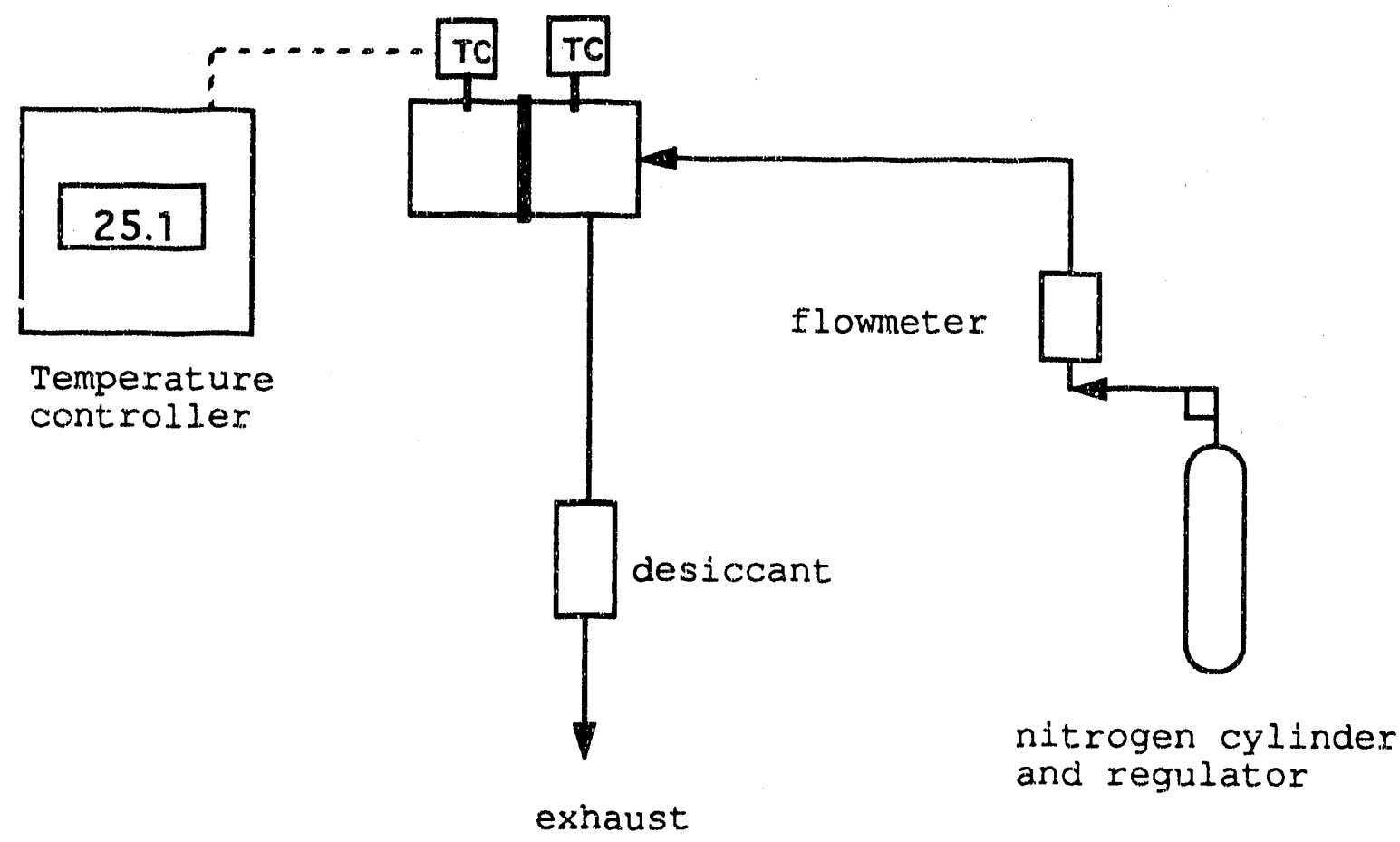

Figure 2. Diffusion cell apparatus. 
from the weight of absorbed water. The flow rate of nitrogen was measured and used to determine the partial pressure of water in the bulk. The pressure was about 1 bar, and the temperature maintained close to $25^{\circ} \mathrm{C}$. The membranes received the same pretreatment described in chapter 4.

At steady state, the flux of water through the membrane is equal to the rate of mass-transfer into the gas phase. Whence,

$$
N_{0}=-\frac{\rho D_{0}}{\left(1-\omega_{0}\right) M W_{0}} \frac{d \omega_{0}}{d y}=-k_{G}\left(p_{i}-p_{b}\right),
$$

where $p_{i}$ is the partial pressure of water at the gas-membrane interface. Furthermore, we assume that equilibrium exists at the interface between the membrane and gas;

$$
p_{0}^{*}=K \omega_{0}^{*}
$$

Therefore, for a membrane of thickness $\ell_{1}$,

$$
N_{0}=-\frac{\left(\omega_{0}^{\prime}-p_{b} / K\right)}{\frac{\ell_{1} M W_{0}}{\bar{D}}+\frac{1}{k_{G} K}}
$$

where $\bar{D}$ is an average of $\rho D_{0} /\left(1-\omega_{0}\right)$ over the concentration range, and $\omega_{0}^{\prime}$ is the mass fraction of water in the membrane in equilibrium with the liquid water. Experimental data are analyzed using 


$$
\frac{\omega_{0}^{\prime}-P_{b} / K}{N_{0}}-\frac{\ell_{1} M W_{0}}{\bar{D}}+\frac{1}{k_{G} K} .
$$

Measurement of the flux of water at different values of $l_{1}$ will give a slope that is inversely proportional to the integral diffusion coefficient $\bar{D}$.

Experimental results for one to three membranes are shown in table 1; figure 3 shows the variable on the left side of equation 7 plotted against the thickness of the membranes. The calculated values for $\bar{D}$ are given in tabie 2 .

Table 1.

\begin{tabular}{|c|c|c|}
\hline \# of membranes & $\mathrm{J}_{0} \times 10^{7} \mathrm{~mol} / \mathrm{cm}^{2} \cdot \mathrm{s}$ & $p_{b} \times 1.0^{5} \mathrm{bar}$ \\
\hline \multicolumn{3}{|c|}{ Run $\# 126.7^{\circ} \mathrm{C}$} \\
\hline 1 & 10.5 & 1107 \\
\hline 2 & 10.2 & 992 \\
\hline 3 & 9.17 & 887 \\
\hline \multicolumn{3}{|c|}{ Run $\# 221.7^{\circ} \mathrm{C}$} \\
\hline 1 & 9.04 & $\overline{1030}$ \\
\hline 2 & 8.32 & 946 \\
\hline 3 & 7.79 & 886 \\
\hline \multicolumn{3}{|c|}{ Run $\# 324.0^{\circ} \mathrm{C}$} \\
\hline 1 & 10.9 & 1052 \\
\hline 2 & 9.42 & 912 \\
\hline 3 & 8.59 & 832 \\
\hline
\end{tabular}

Table 2.

\begin{tabular}{|c|c|}
\hline run $\#$ & $M W / \bar{D} \times 10^{-6} \mathrm{~cm} \cdot \mathrm{s} / \mathrm{mol}$ \\
\hline 1 & 1.25 \\
2 & 0.996 \\
3 & 1.46 \\
\hline
\end{tabular}




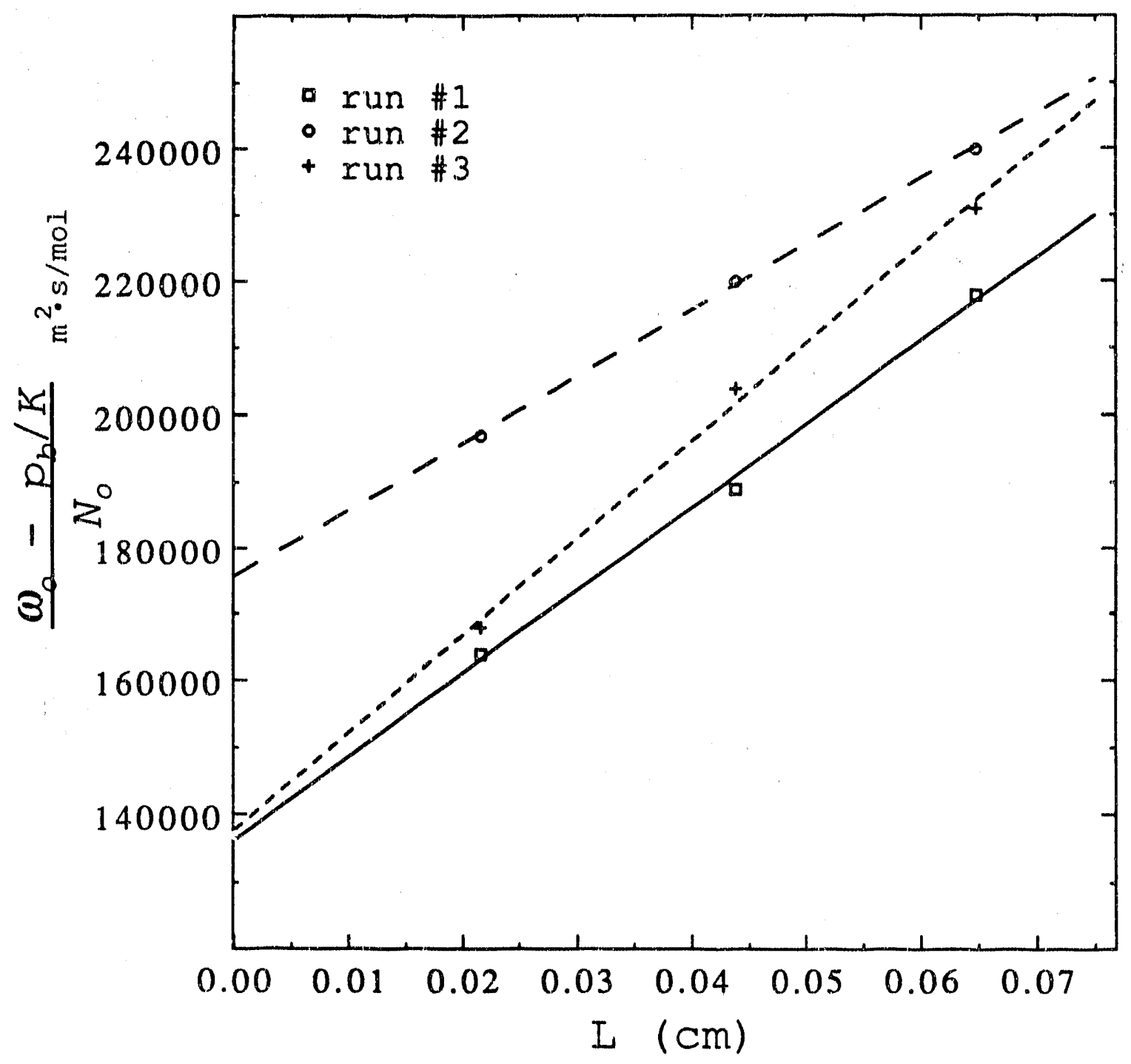

Figure 3. Variable defined in equation 7 for different membrane thicknesses. 
Although crude, the experiments do give us an approximate value of the diffusion coefficient, $D_{0} \approx 1.0 \times 10^{-5} \mathrm{~cm}^{2} / \mathrm{s}$. It is clear from figure 3 that most of the resistance is in the gas phase and not the membrane. Most of the errors associated with this method, however, would tend to lower the value of the measured diffusion coefficient.

In the second approach, five membranes were placed in series in the cell. At steady state, the flux of water is measured as described above. The concentration profile is determined by disassembling the cell and separating the layers of membranes. Then, each membrane is weighed and its dry weight is subtracted. The diffusion coefficient follows directly from equation 2. The water profiles for two experimental runs are shown in figure 4. The results for two different gas flow rates are shown in table 3 . In both experiments the temperature was $27.0^{\circ} \mathrm{C}$. These experiments gave higher values, $D_{0} \approx 3 \times 10^{-5} \mathrm{~cm}^{2} / \mathrm{s}$, for the diffusion coefficient.

\subsection{Conductometric method}

The conductometric method is is reviewed below. More details can be found in reference [11] and [14]. Because it is an elegant technique, we considered its application to the polymer-electrolyte.

Table 3.

\begin{tabular}{|c|c|c|}
\hline run \# & $\mathrm{J} \times 10^{7} \mathrm{~mol} / \mathrm{cm}^{2} \cdot \mathrm{s}$ & $M W / \bar{D} \times 10^{-6} \mathrm{~cm} \cdot \mathrm{s} / \mathrm{mol}$ \\
\hline 1 & 8.00 & 2.61 \\
\hline 2 & 13.07 & 2.35 \\
\hline
\end{tabular}




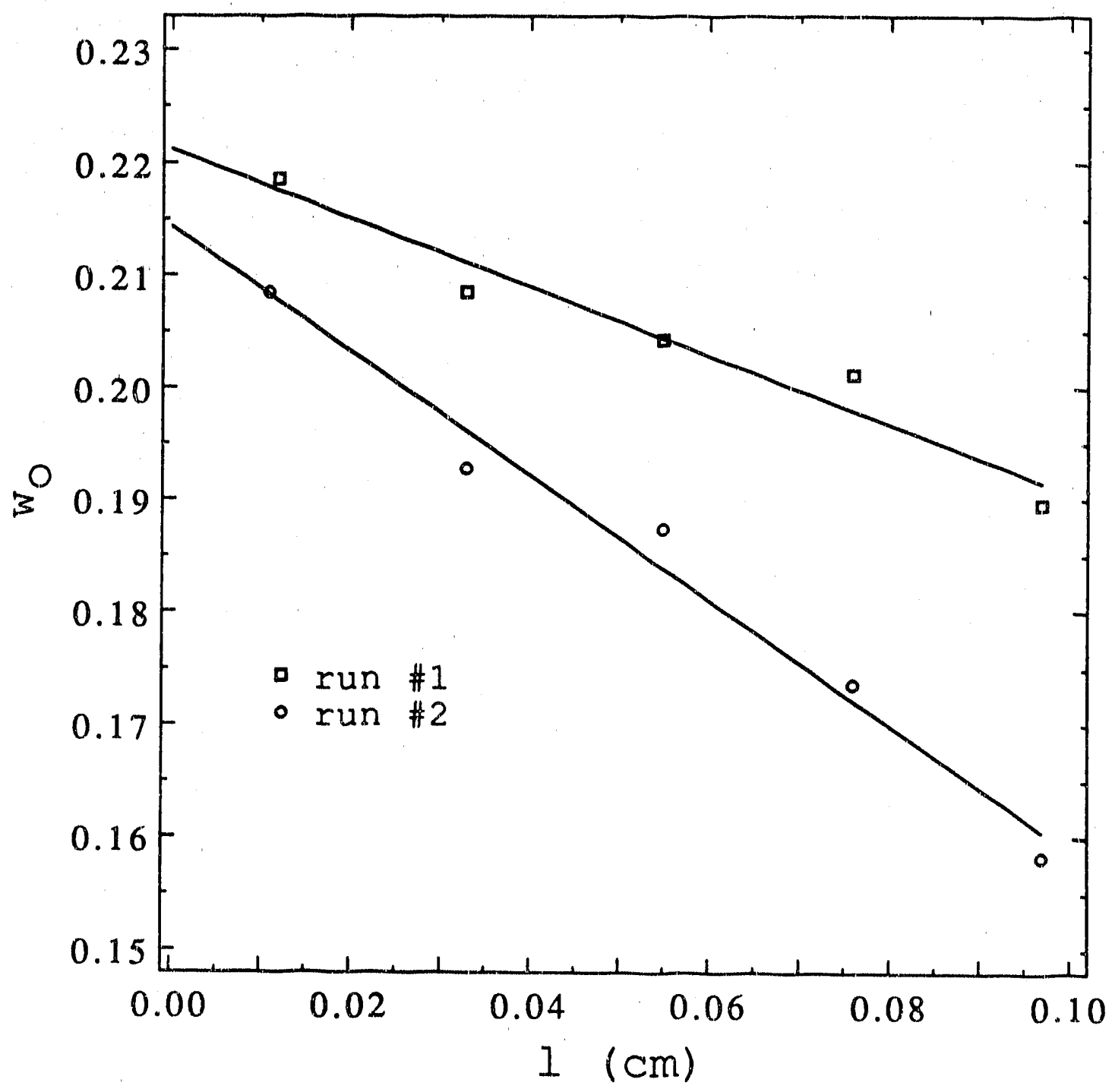

Figure 4. Water profile in diffusion cell. Temperature $27^{\circ} \mathrm{C}$. 
Figure 5 shows a conductometric cell. A membrane was sandwiched between two plastic blocks. Electrodes were placed one sixth of the way from each end and are used to measure the conductivity across the membrane at these two locations. An arbitrary concentration profile is established within the membrane. At the start of the experiment both ends are sealed off. The differential equation, initial conditions, and boundary conditions are given below:

$$
\frac{\partial c}{\partial t}-D \nabla^{2} c
$$

$$
\nabla_{c}=0 \text { at } x=0 \text { and } x=a \text {, }
$$

$$
\text { and } c(x, 0)-f(x) \text {. }
$$

The Fourier series solution is

$$
c(x, t)=c_{0}+\sum_{n=1}^{\infty} B_{n} e^{-\frac{D n^{2} \pi^{2} t}{a^{2}}} \cos \left\{\frac{n \pi x}{a}\right\} .
$$

Al1 of the coefficients for even values of $n$ vanish because of the boundary conditions. The placement of the electrodes results in the third term in the series being zero. Since the series converges quickly, at long times

$$
\frac{d \ln \left(c_{a / 6}-c_{5 a / 6}\right)}{d t}=-\frac{\pi^{2} D}{a^{2}}
$$



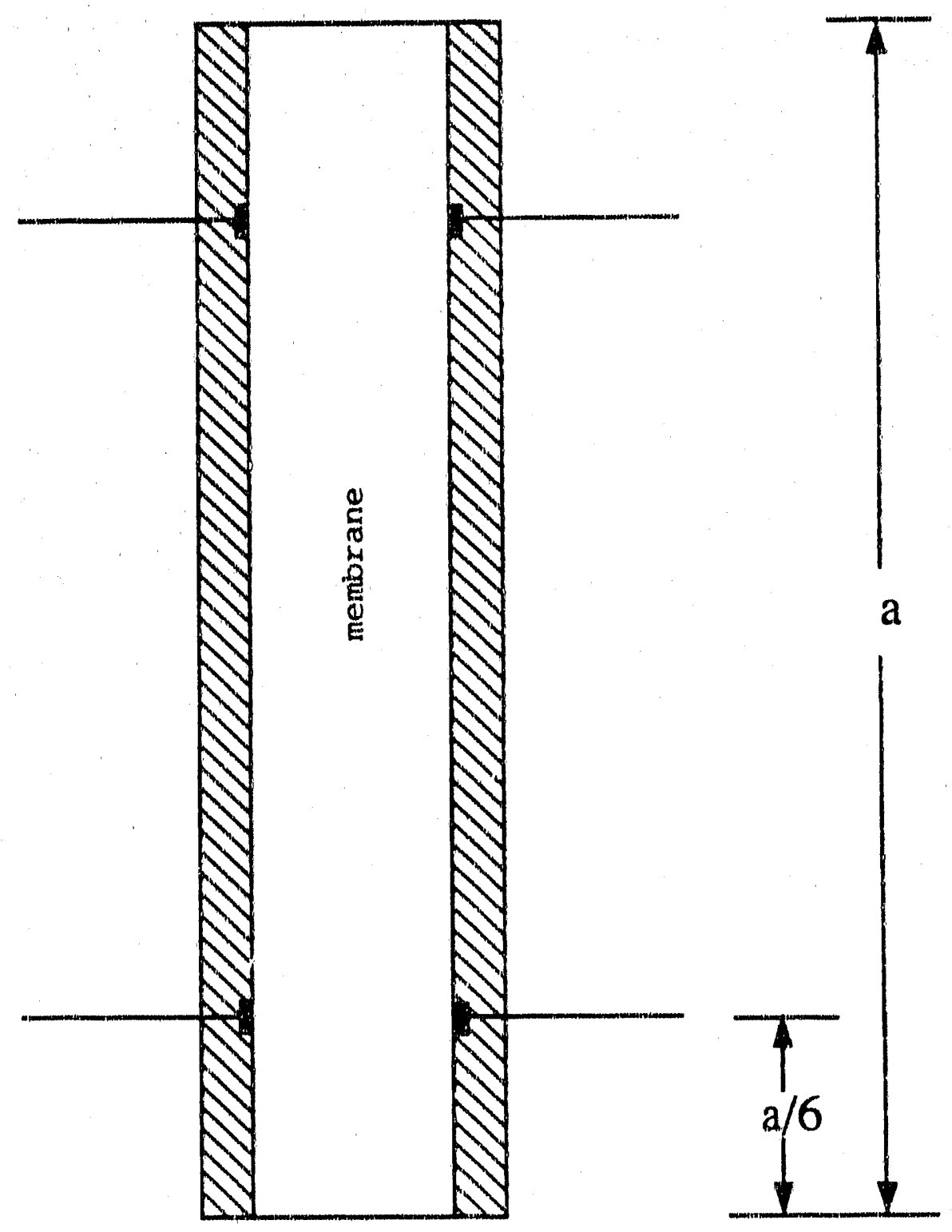

Figure 5. Conductometric cell. Electrodes placed a/6 from each end. 
The diffusion coefflcient is for the value of the final concentration $c_{0}$. Because of difficulties measuring the conductivity, this teath. nique did not prove to be successful. This will be discussed further in chapter 7 .

\subsection{Mutual and Intra Diffusion Coefflcients}

Keller et al. [15] experimentally examined the relation between mutual and tracer diffusivities in terms of dilute solution theory. The differences between the mutual and intra-diffusion coeficients can be seen most clearly within the framework of concentrated solution theory, which is based on the multicomponent diffusion equation,

$$
c_{i} \nabla \mu_{i}-\sum_{j \rightarrow 1}^{n} K_{i j}\left(v_{j}-v_{i}\right) .
$$

In dilute solution theory, the only important interactions occur between solute and solvent. In contrast, the phenomenological equations of concentrated solution theory account for interactions among all the species through the frictional coefficients $K_{i j}$.

The use of self-diffusion measurements for membranes and other applications of concentrated solution theory has been discussed by Newman [16] and Bennion [17]. The use of radioactivuly tagged species and Nuclear Magnetic Resonance techniques to measure selfdiffusion coefficients is common. Tyrrell and Harris [18] provide a theoretical discussion of both classical methods and spin-echo rech. niques for measuring diffusion coefficients using MMR. A description 
of the diffusion processes and the relation between diffusion coefficients in terms of irreversible thermodynamics is given by de Groot and Mazar [19].

The "tagged" molecules in NMR or radiotracer experiments represent additional species which must be inciuded in the multicomponent diffusion equations. That is, the interactions between the tagged and untagged species can have significant effect on the diffusion prociess. Unless this can be estimated or measured independently, these interactions will complicate the determination of the transport properties.

Even in "thought experiments," where the species truly are identical, one species moving to the right by thermal motion will see another molecule moving to the left. In dilute solution theory, the only interactions considered are between species $i$ and the solvent. whereas in concentrated solution theory all interactions are important.

Consider diffusion of a tagged water molecule through the membrane. From equation 11 we can write

$$
c_{0}^{*} \nabla_{0}^{*}=K_{00}^{*}\left(v_{0}-v_{0}^{*}\right)+K_{o m}^{*}\left(v_{m}-v_{0}^{*}\right)+K_{0+}^{*}\left(v_{+}-v_{0}^{*}\right)
$$

for the tagged species. Furthermore we will assume that,

$$
D_{i, j}=D_{i j}^{*}
$$

and by continuity $N_{0}=-N_{0}^{*}$, and $v_{m}=v_{+}=0$. Equation 12 can be 
written as,

$$
\left.\mathrm{N}_{0}^{*}=c_{0}^{*} v_{0}^{*}-\frac{-c_{0}^{*} \nabla_{0}^{*}}{c}\left\{1+\frac{c_{0}^{*}}{c_{0}}\right] \frac{c_{0}}{D_{00}^{*}}+\frac{c_{m}}{D_{o m}}+\frac{c_{+}}{D_{0+}}\right\} .
$$

The flux of the tagged species can also be written as

$$
\mathrm{N}_{0}^{*}-\alpha_{0}^{*} \nabla_{\mu_{0}^{*}}^{*}
$$

Combining equation 14 and 15 yields

$$
\alpha_{0}^{*}-\frac{c}{\frac{c_{0}+c_{0}^{*}}{D_{o 0}^{*}}+\frac{c_{m}}{D_{o m}}+\frac{c_{+}}{D_{O+}}}
$$

In the case where there axe no tagged species, we can develop a similar relation. The multicomponent diffusion equation for water is

$$
c_{0} \nabla \mu_{0}-K_{o r n}\left(v_{m}-v_{0}\right)+K_{0+}\left(v_{+}-v_{0}\right)
$$

and thexefore

$$
\alpha_{0}=\frac{c}{\frac{c_{m}}{D_{\text {on }}}+\frac{c_{t}}{D_{\text {ot }}}}
$$

Clearly, the tagged molecules adi an additional species to the system. The flux of the tagged spectes will depend on another term reflecting the interactions between the tagged species and water. In 
order to use the self-diffusivity, one would need to estimate $D_{00}$. From equations 16 and 18 , and further assuming that the concentration of tagged water is small, we can relate the two diffusion coefficients;

$$
\alpha_{0}=\alpha_{0}^{*}\left\{\frac{\frac{c_{0}}{D_{o 0}^{*}}+\frac{c_{m i}}{D_{o m}}+\frac{c_{+}}{D_{o+}}}{\frac{c_{m}}{D_{o m}}+\frac{c_{+}}{D_{o+}}}\right\}
$$

From equation 19 one sees that $\alpha_{0}^{*}-\alpha_{0}$ only when the concentration of water goes to zero.

On a molecular level, the difference is interpreted as follows. We consider two species in the diffusion process, the membrane and water. The measurable diffusion event is the interchange of molecules of membrane and water. The only interactions are those between water and membrane. Thus, a single diffusion coefficient, the mutual-diffusion coefficient, is required.

On the other hand, with NMR or radio-tracer techniques, the introduction of a labeled species creates a ternary system. Three diffusion coefficients are now required to describe transport processes. There will be interactions between the water and membrane, between the labeled water and nembrane, and between the labeled and unlabeled water. The intramdiffusion coefficient of water in the membrane given by NMR is a measure of the movement of "labeled" water in a homogeneous medium. The labeled water interacts 
with both the membrane and other water molecules. At high water concentrations, the interactions between the two types of water are significant. As the total concentration of water is reduced, the interactions between labeled water and unlabeled water become negligible; and if isotope effects are small, the measured intra-diffusion coefficient will be equal to the mutual-diffusion coefficient.

\subsection{Calculation of BInary Diffusion Coefficlents}

The binary interaction coefficients are related to the frictional coefficients by

$$
K_{i j}=\frac{R T c_{i} c_{j}}{c D_{i j}}
$$

We described how to relate the binary interaction coefficients and the frictional coefficients to measurable quantities in chapter 2. There are three transport properties required: the diffusion coefficient of water, the electrical conductivity, and the transport number of water.

Springer et al. [20] measured the electrical conductivity of Nafion 117 membrane at various temperatures and water contents. They gave the following fit of their data

$$
\kappa=(0.005139 \lambda-0.00326) \exp \left(1268\left(\frac{1}{303}-\frac{1}{T}\right)\right] .
$$


From the experiments described in this chapter and the dependence on level of hydration from [10], we estimate the mutual diffusion coefficient of water as

$$
D_{0}=3.5 \times 10^{-2} \exp \left(\frac{-2436}{T}\right) \frac{\lambda}{14} \mathrm{~cm}^{2} / \mathrm{s} \text {. }
$$

The activation energy was taken from the expeximents of Yeo and Eisenberg. [9]

Analysis of the potentials of cells with transference, as described in chapter 4, gave an estimation of the transport number of water. Assuming that the transport number of water was independent of temperature, we developed the expression

$$
\frac{t_{0}^{m}}{z_{0}}=\left(\frac{1}{(0.35 \lambda)^{4}}+\frac{1}{(1.47)^{4}}\right)^{-1 / 4}
$$

The density was estimated from information provided by the manufacturer of Nafion membranes. The most important factor in determining the physical properties of the membrane is the equivalent weight (EW). The equivalent weight is defined as the weight of acid polymer that will neutralize one equivalent of base. This is related to the ion-exchange capacity (IEC) by,

$$
I E C=\frac{1000}{E W}
$$

For the fuel-cell system, there are only three components. Assuming the electrolyte is electrically neutral and that we know the 
properties of the dry membrane, i.e. density and (IEC), we can obtain the concentration of each species from the density. The water content in the membrane will determine the concentrations of each species in the membrane, and this relationship will be used in the model. DuPont reports that the dry polyner has a density of $\rho-1.98 \mathrm{~g} / \mathrm{cm}^{3}$, and that the volume increase for water absorption is about twice the weight increase. This leads to the following expression for the density:

$$
\rho=\frac{1.98+0.0324 \lambda}{1+0.0648 \lambda}
$$

For this analysis, the molecular weight is taken to be identical to the equivalent weight. The concentration of water can be expressed as

$$
c_{0}-\frac{1980 \lambda / E W}{1+0.0648 \lambda}
$$

To ensure $K_{+m}$ is positive,

$$
D_{0}>\frac{\left(1-\omega_{0}\right) M W_{0}}{\rho} \frac{\kappa \xi}{F^{2}}(\lambda-\xi) \frac{d \mu_{0}}{d \omega_{0}} .
$$

Using the thermodynamic data of Zawodzinski et al. [10], we estimated the activity factor as

$$
\frac{d \mu_{0}}{d \omega_{0}}=0.04 R T\left(\frac{1}{\omega_{0}^{2}}+1\right)
$$


From the data given above, the solid line in figure 6 shows the minimum value of the diffusion coefficient consistent with equation 27. The diffusion coefficients from our analysis, equation 22, as well as those reported in references [10], are inclided in this figure.

The frictional coefficients, $K_{i j}$, at $80^{\circ} \mathrm{C}$, are shown in figure 7. The binary-interaction coefficients, $D_{i j}$, are shown in figures 8 anı 9 for 80 and $25^{\circ} \mathrm{C}$ respectively. In general, as the membrane is hydrated, the diffusion coefficients increase, and the frictional coefficients decrease. The frictional coefficients and the binaryinteraction coefficients are strongly dependent on composition; and as we surmised in chapter 2 , there is no clear advantage to using $D_{i j}$ s.

\section{List of Symbols}

$c$

total concentration, mol/1

$c_{i}$

concentration of species $i$, mol/l

$d_{i}$

driving force for transport of species $i, N / \mathrm{m}^{3}$

Do

mutual diffusion coefficient of water, $\mathrm{cm}^{2} / \mathrm{s}$

$D_{i . j}$

binary-interaction coefficient of species $i, \mathrm{~cm}^{2} / \mathrm{s}$

F

Faraday's constant, $96,487 \mathrm{C} / \mathrm{eq}$

$\mathrm{J}_{i}$

flux of species $i, \operatorname{mol} / \mathrm{cm}^{2} \cdot s$

${ }_{G}$

gas-phase-mass transfer coefficient, $\operatorname{mol} / \mathrm{bar} \cdot \mathrm{m}^{2} \cdot \mathrm{s}$

$k^{r}$

partition coefficient defined by equation 5 


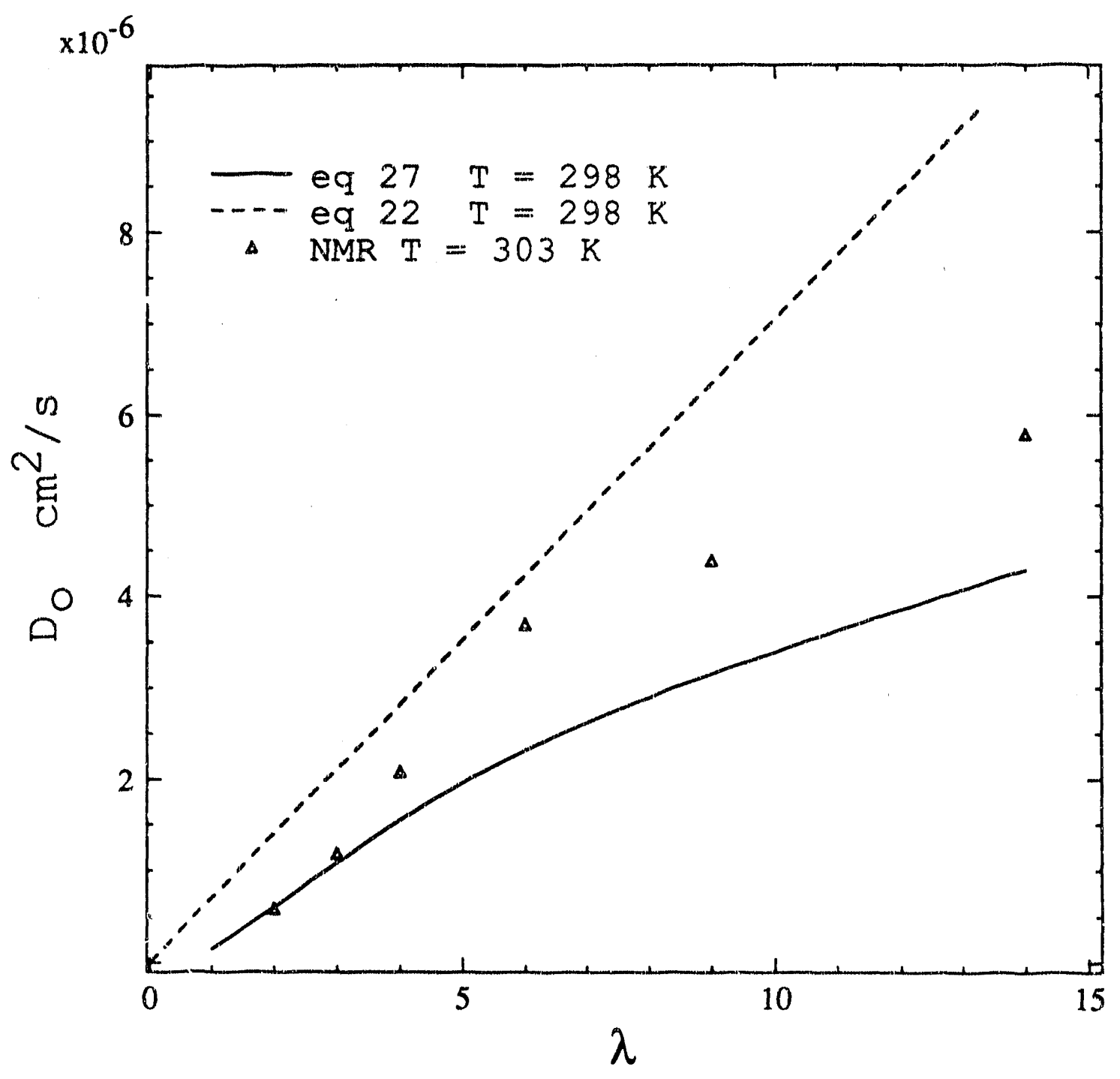

Eigure 6. Diffusion coefficient of water in Nafion 117 membrane. The solid line is the minimum value calculated from equation 27; the dashed line is our estimate, equation 22; and the triangles are experimental data of Zawodzinski et al [10]. 


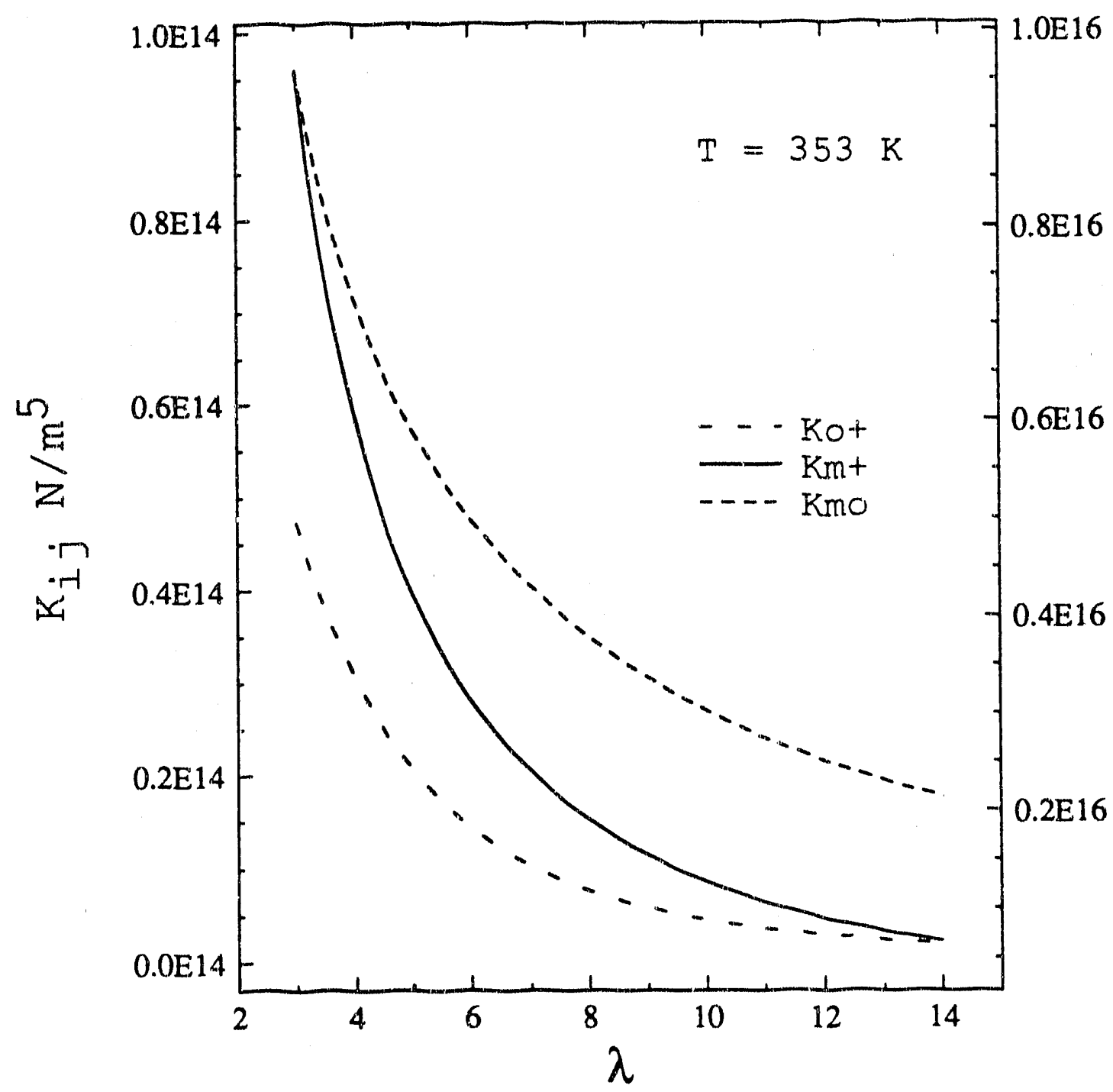

Figure 7. Frictional coefficients $k_{i j}$ calculated from the three transport properties: $\kappa, D_{0}$, and $\xi$. 


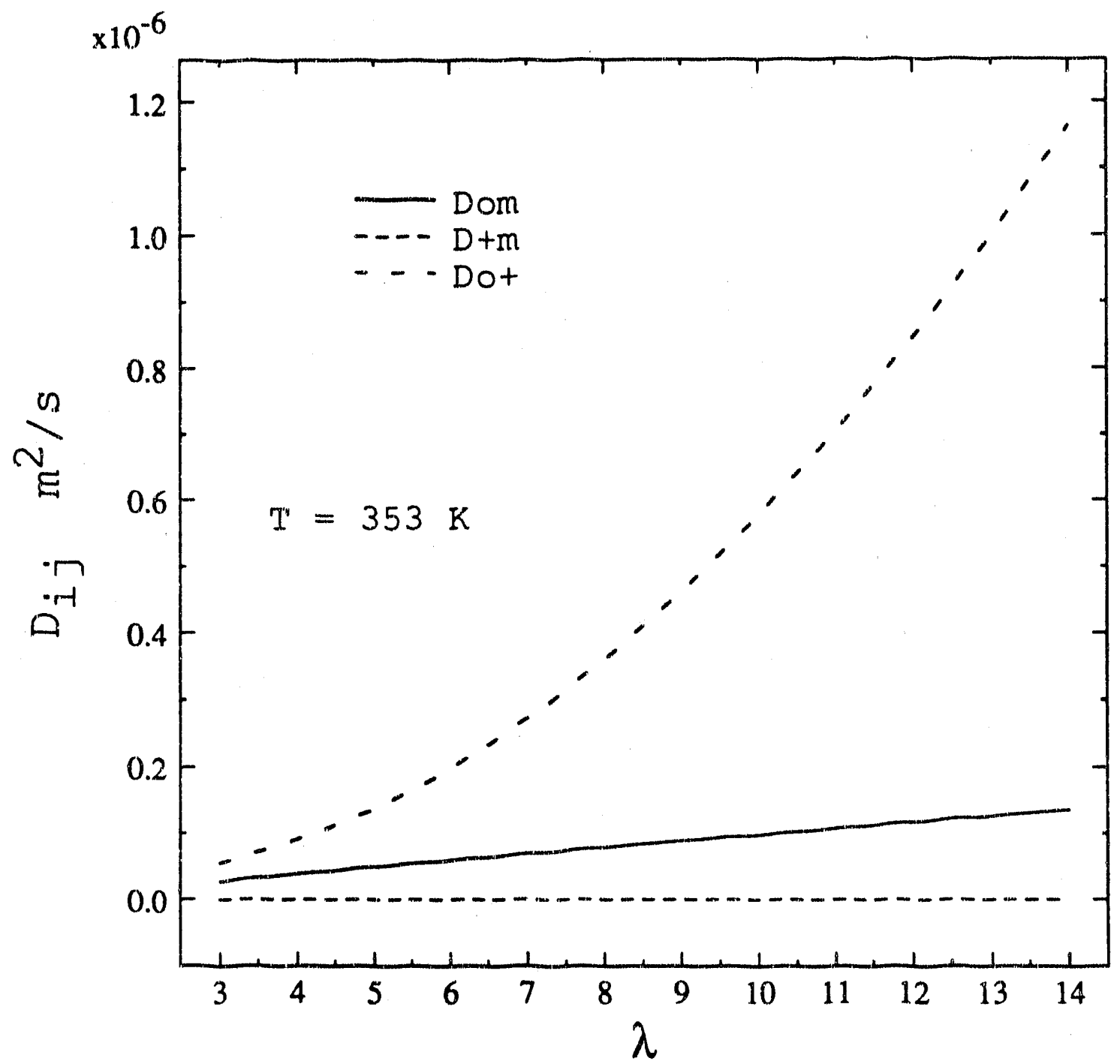

Figure 8. Binary-interaction coefficients, $D_{i j}$, calculated from the three transport properties: $\kappa, D_{0}$, and $\xi$. 


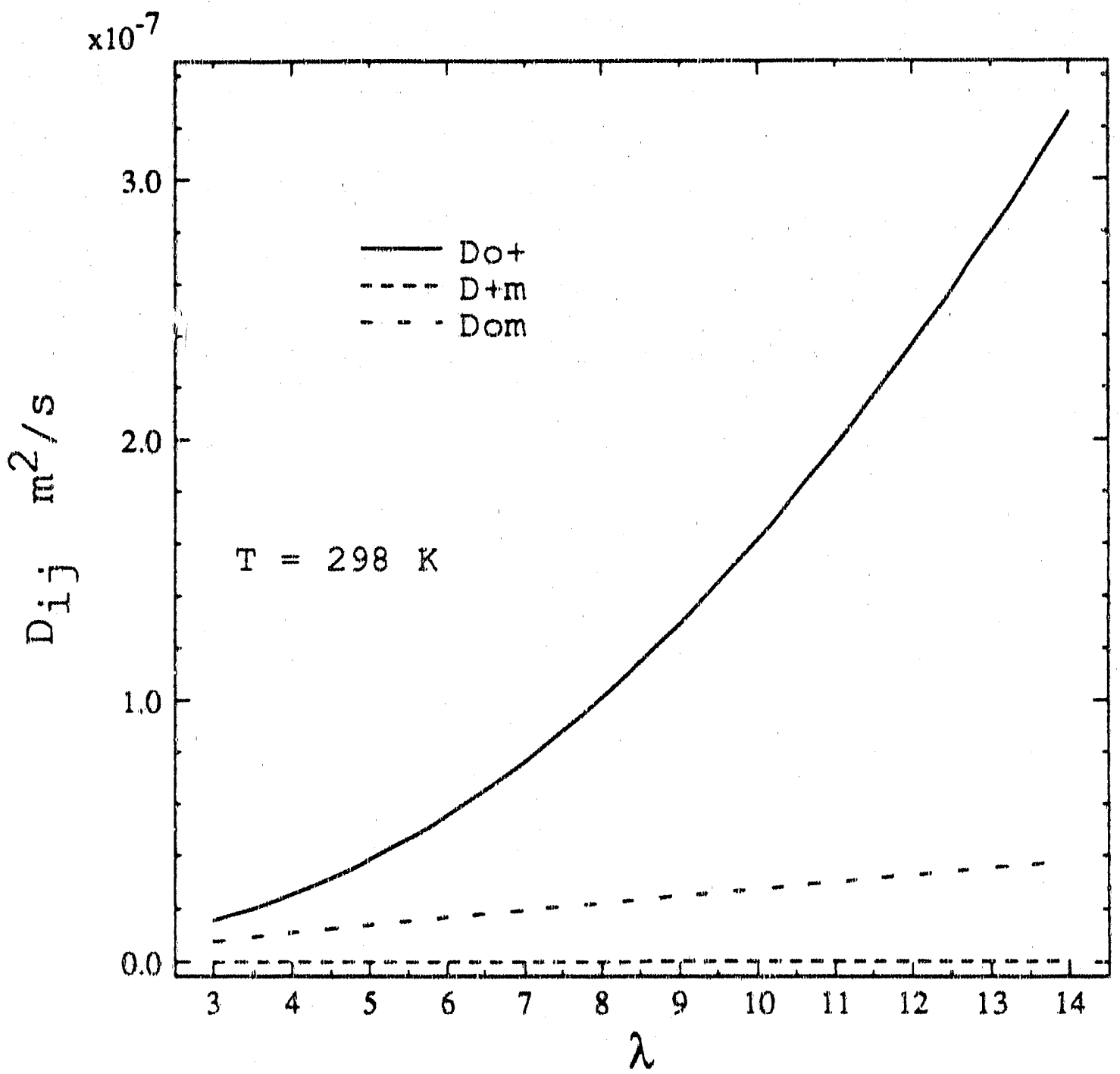

Figure 9. Binary-interaction coefficients, $D_{i j}$, calculated from the three transport properties: $K, D_{0}$, and $\xi$. 


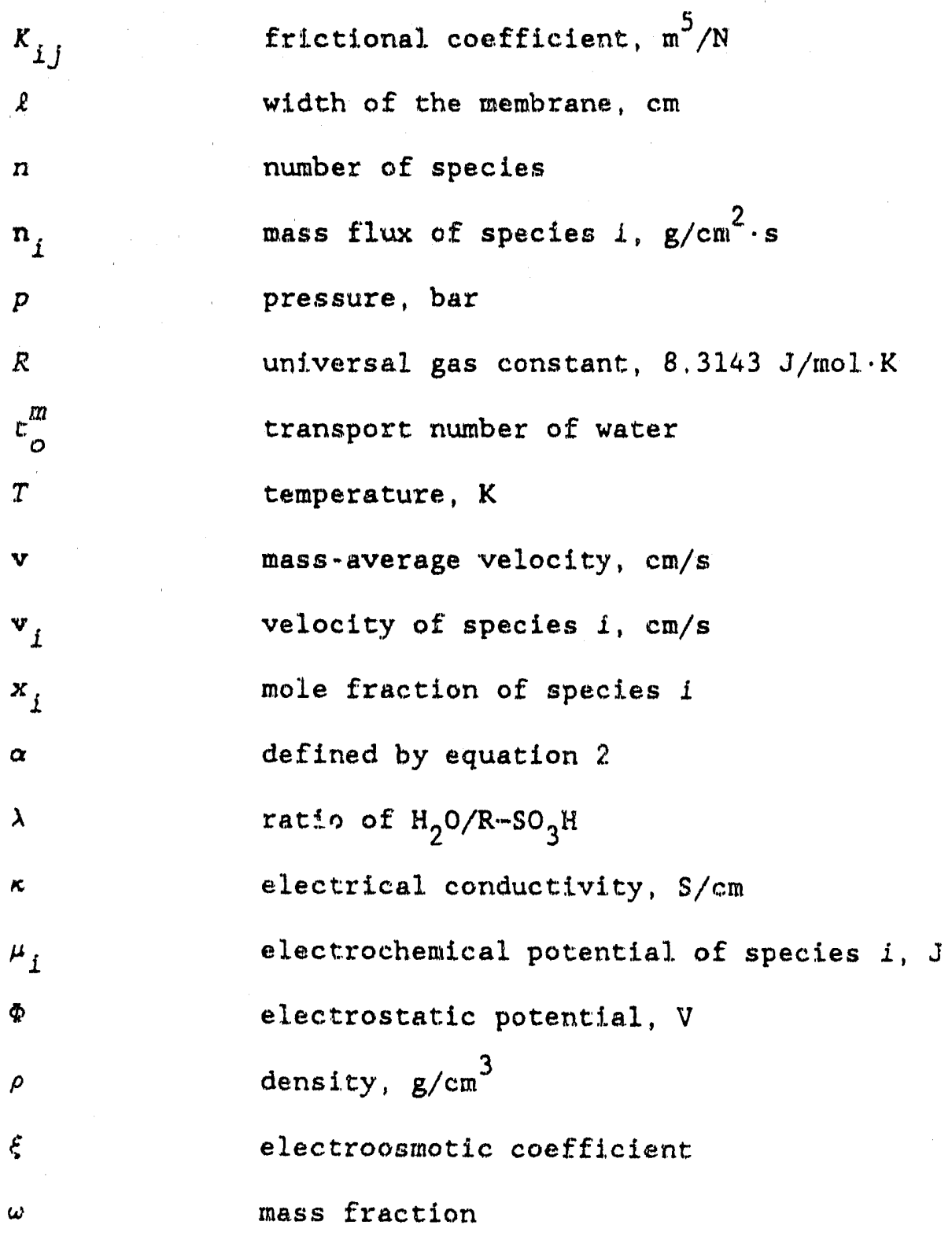

Subscripts

$+\quad$ hydrogen ion

D

water

m

nembrane 
tagged species

\section{References}

[1] H. L. Yeager, "Transport Properties of Perfluorinated Polymer Membranes," in Perfluorinated Ionomer Membranes, edited by A. Eisenberg and H. L. Yeager, Amer. Chem. Soc. Symposium Series No. 180, Washington, D.C. (1982).

[2] Membranes and Ionic and Electronic Conducting Polymers, proceedings Volume 83-3, edited by E. B. Yeager, B. Schumm, Jr., K. Mauritz, K. Abbey, D. Blankenship, and J. Akridge, The Electrochemical Society, Inc., Pennington, N. J. (1983).

[3] R. S. Yeo, "Ion Clustering and Proton Transport in Nafion Membranes and Its Applications as Solid Polymer Electrolyte," $J$. Electrochem. Soc., 130, 533-538 (1983).

[4] H. Kimikuza, K. Kalbara, E. Kumamoto, "Diffusional and Conductive Membrane Permeabilities in the Cation Exchange MembraneAqueous Electrolyte System," J. Membrane Sci., 4, 81-98 (1.978).

[5] R. C. T. Slade, A. Hardwick, and P. G. Dickens, "Investigations of $\mathrm{H}$ Motion in Nafion Films by Pulsed NMR and A.C. Conductivity Measurements," Solid State Ionics, 9\&10, 1093-1098 (1.983).

[6] H, L. Yeager and A. Steck, "Cation and Water Diffusion in Nafion Ion Exchange Membranes: Influence of Polymer Structure," Elec. trochemical Science and Technology, 128, 1880-1884 (1981). 
(7) M. Verbrugge, "Methanol Diffusion in Perfluorinated IonExchange Membranes," J. Electrochem. Soc., 136, 417-423 (1989).

[8] M. W. Verbrugge and R. F. Hill, "Analysis of Promising Perfluorosulfonic Acid Membranes for Fuel-Cell Electrolytes," J. Electrochem. Soc., 137, 3770 3777 (1990).

[9] S. W. Yeo and A. Eisenberg, "Physical Properties and Supermolecular Structure of Perfluorinated Ion-Containing (Nafion) Polymers," J. AppI. Polymer Sci., 21, 875-898 (1977).

[10] T. A. Zawodzinski, Jr., M. Neeman, L. O. Sillerud, and S. Gottesfeld, "Determination of Water Diffusion Coefficient in Per. fluorinated Ionomeric Membranes," J. Phys. Chem., 95, 6040-6044 (1991).

[11] R. A. Robinson and R. H. Stokes, Electrolyte Solutions, Butterworths, London (1968).

[12] J. Crank, The Mathematics of Diffusion, Claredon Press, Oxford (1.956).

[13] R. Byron Bird, Warren E. Stewart, and Edwin N. Lightfoot, Transport Phenomena, John Wiley \& Sons, Inc., New York (1960).

[14] T. W. Chapman, Ph. D. dissertation, University of California, Berkeley, CA (1967).

[15] Kenneth H. Keller, Edgardo R. Cánales, and Su Il Yum, "Tracer and Mutual Diffusion Coefficients of Proteins," J. Phys. 
Chem. , 75, 379 (1971).

[16] John Newman, Electrochemical Systems, 2nd edition, Prentice-Hall Inc., Englewood Cliffs, N.J. (1991).

[17] D. N. Bennion, Mass Transport of Binary Electrolyte Solutions in Membranes, Water Resources Center Desalination Report No. 4, Report No. 66-17, University of California, Los Angeles, CA (1966).

[18] H. J. V. Tyrrell and K. R. Harris, Diffusion in Liquids, Butterworths, Cambridge University Press (1984).

[19] S. R, de Groot and P. Mazur, Non-Equilibxium Thermodynamics, Dover Publications, New York (1984).

[20] T. E. Springer, T. A. Zawodzinski, and S. Gottesfeld, "Polymer Electrolyte Fuel Cell Model," J, Electrochem. Soc., 138, $2334-2342$ (1991). 
Chapter 6

Steady-state Results and Water Management

\subsection{Introduction}

Based on the model developed in chapter 3 , we present a steadystate analysis of transport in a solid-polymer-electrolyte fuel cell. Nguyen et al. [1] raise heat-transfer design issues in polymerelectrolyte fuel cells; but, by and large, the subject has not been treated in the literature. Nevertheless, as will be shown in this chapter, thermal effects must be considered in the analysis of water management.

We consider a membrane-electrode assembly operating at steady state on air and reformed methanol. The fuel and air streams are heated and humidified prior to entering their respective channels where they are consumed in electrochernical reactions. Hydrogen gas diffuses through the porous electrode and reacts at the anode in a three-phase region containing polymer electrolyte, gaseous reactants, and carbon matrix including platinum catalyst. Oxygen passes through the gas-diffusion electrode to the cathode. At the cathode, the hydrogen ions react with oxygen at similar catalyst sites to form water. Water also absorbs into or evaporates from the membrane depending on the partial pressure of water.

We examined three issues with respect to water management: 1) net transport of water, which must be supplied or recycled thereby reducing the overall efficiency of the cell, from the anode to the 
cathode, 2) dehydration of the anode leading to high ohmic losses because the conductivity of the polymer electrolyte is a strong function of the degree of hydration, and 3) flooding of the cathode.

It is common practice to supersaturate the inlet gases with water prior to entering the fuel cell. Since water is produced at the cathode, it is not ubvi, 's that additional water need be supplied to the fuel cell. We hope to elucidate when and why additional water might be needed to enhance the performance.

In addition, the utilization of hydrogen is an jmportant factor in the optimization of these fuel cells. One could circumvent water and thermal management problems by increasing the flow rates of the gas streams. This will, however, affect the utilization of fuel, net transport of water across the cell, and the overall efficiency of the fuel cell.

Because the effect of changes in individual properties (conductivity for example) seems evident, we do not attempt to make a parametric study. Instead, using the best available data, we examine the complex relationship between operating conditions and transport processes in the fuel cell. Where possible, the parameters were obtained from our experimental measurements or from data available in the literature. The thermodynamic and kinetic data and the transport properties used in the simulation are sumnarized in appendix $B$; the computer program is listed in appendix $C$.

The analysis is divided into three areas: 1) constanttemperature study of a unit cross section of the fuel cell, 2) water 
management in a membrane-electrode assembly at constant temperature with cocurrent flow of air and fuel, and 3) combined material and energy balance for the membrane-electrode assembly.

\subsection{Unit cross section}

First, we consider a unit cross section of the fuel cell. The temperature is fixed, and we specify the mole fractions of the gaseous species at the ends of the two gas-diffusion electrodes. The current-voltage curve for the conditions of table 1 is shown in figure 1. For all of the simulations the width of the gas diffusion electrodes was $360 \mu \times n$, and the width of the reaction zone was $10 \mu \mathrm{m}$. The open-circuit potential is determined from equation B-12. At low current density, ohmic losses are small, and polarization at the cathode controls the cell potential. As the current density increases, ohmic losses grow, and the curve becomes roughly linear;

Table 1.

Gas composition at the boundaries.

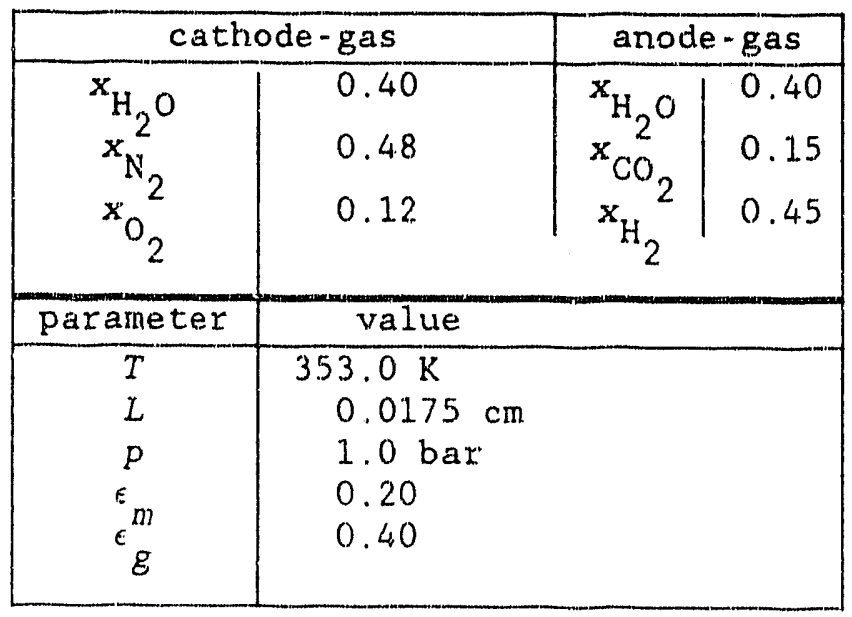




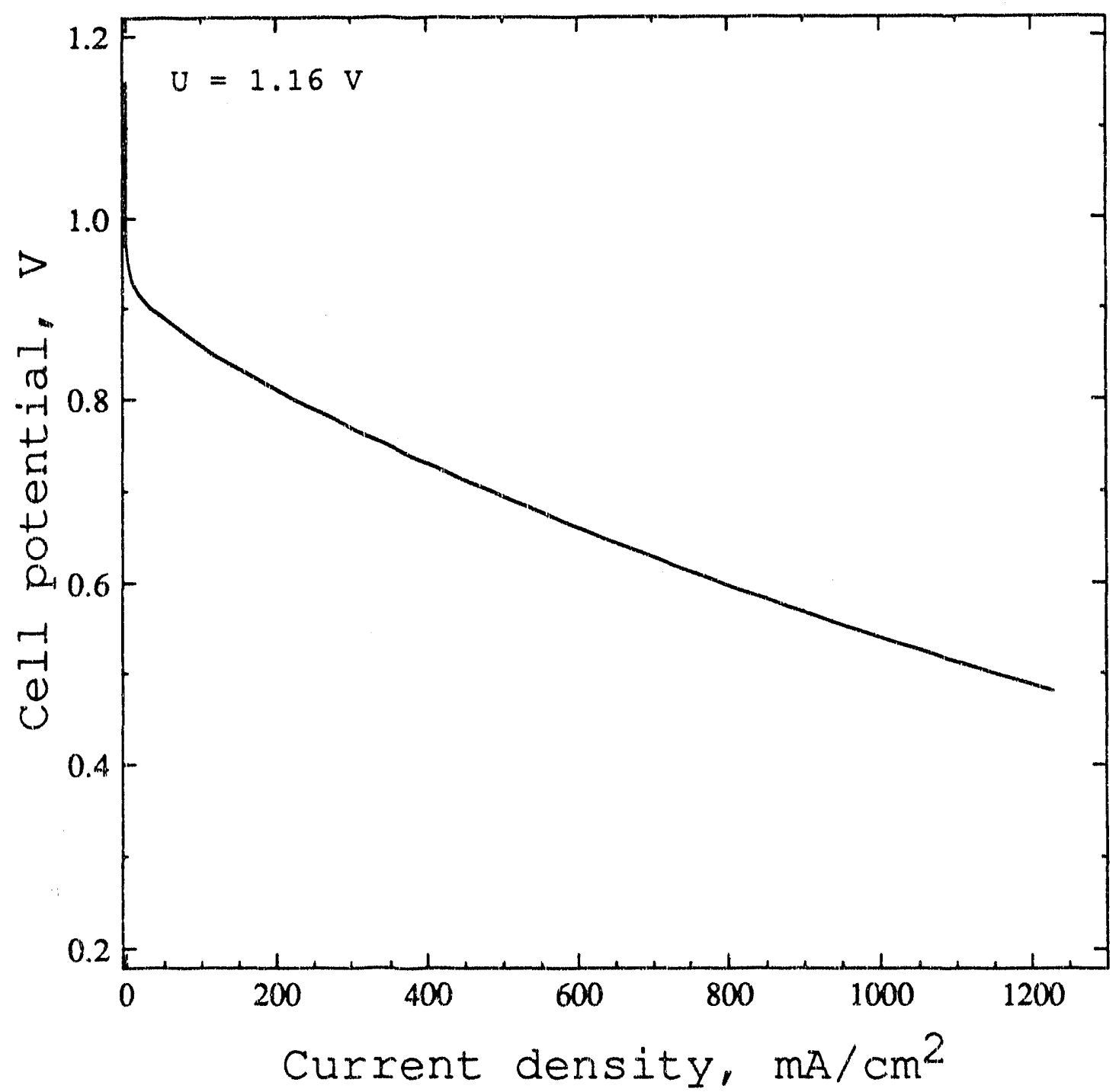

Figure 1. Cell potential versus current density for unit cell. Operating conditions are listed in table 1. 
in this region the slope is inversely proportional to the electrical conductivity. Because the overall water content, which determines the conductivity of the membrane, increases with current density, the curve is slightly concave upward (the water profiles in figure 2 will clarify this effect).

Ticianelli et al. [2] present experimentally measured curves. They observed what appear to be mass-transfer limitations in experi. mental cells, a sharp drop in current density at low cell potentials. The model does not predict mass-transfer limitations in the gasdiffusion electrodes until current densities of about $5 \mathrm{~A} / \mathrm{cm}^{2}$. The model, however, does not include adsorption and mass transfer of hydrogen or oxygen in the water or polymer film, which could limit the current density. The mass-transfer limitations may also be the result of flooding. As the pores fill up with water, the volume fraction of the gas.decreases, and access to the catalyst may be res. tricted.

Figure 2 presents profiles of water content in the membrane at different current densities. $\lambda$ is the ratio of moles of water per sulfonic acid group. At the cathode, water is produced in stoichiometric proportion to the current; and with increasing current, more water is dragged from the anode. A concentration gradient builds up to counteract the elestroosmotic drag.

As the current density grows, one observes an increase in the water content at the cathode and slight dehydration at the anode. Although the flux of water across the membrane is constant, because 


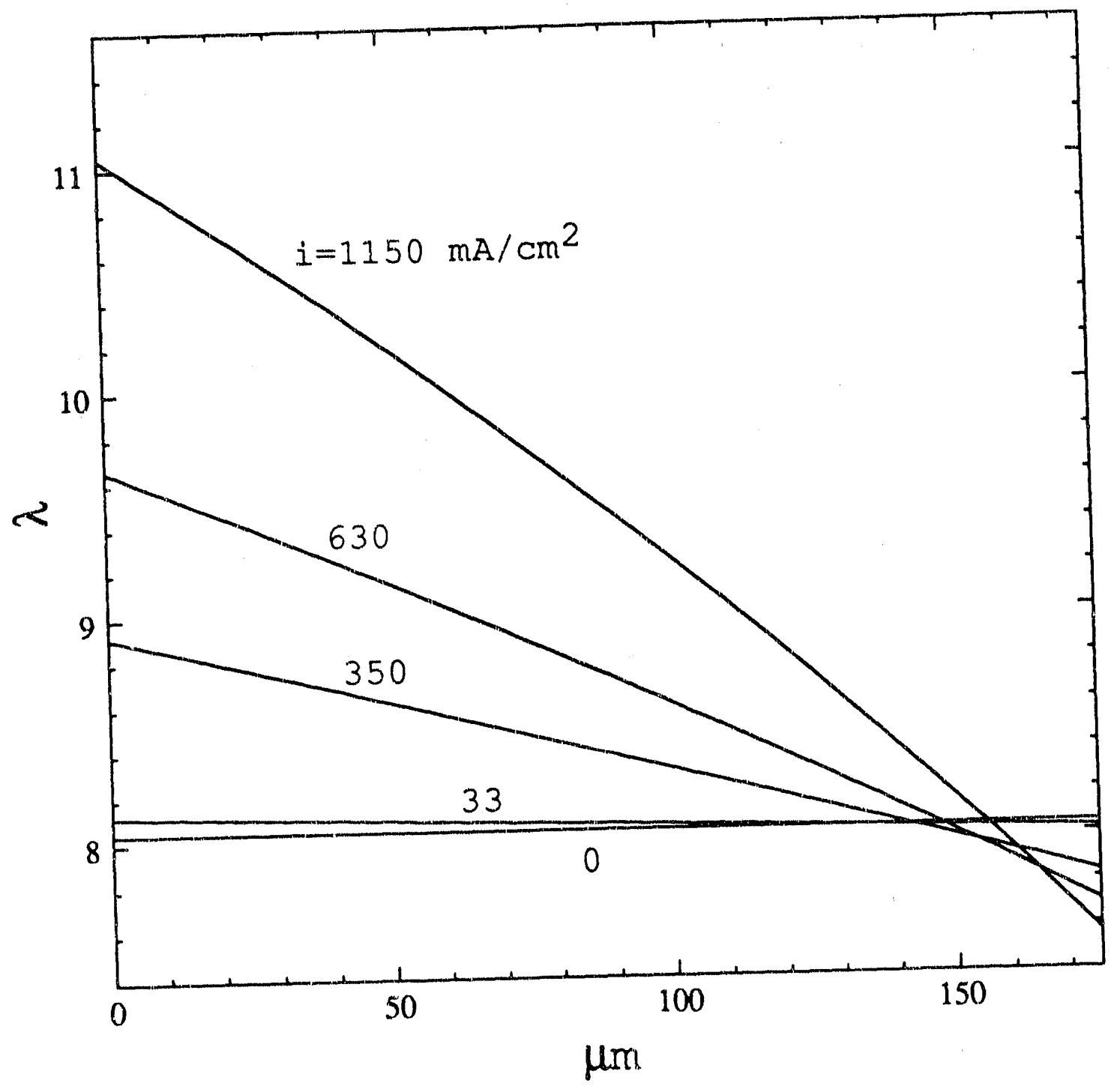

Eigure 2. Water map for unit cell at various current densities. $\quad p=1.0$ bar, $T=353 \mathrm{~K}$. Cathode is on the left; arode on the right. $\lambda$ is the ratio of moles of water per sulfonic acid group. 
of the variable physical properties, the water profiles are not straight. There is little resistance to mass transfer in the gas phase or in the membrane; and consequently, even at high current densities, dehydration of the anode is not significant.

\subsection{Isothermal case}

Next, we consider a two-dimensional membrane-electrode assembly (shown in chapter 3), with cocurrent flow of air and fuel streams. The temperature is constant, but as the gas streams flow down the channels, fuel and oxygen are consumed, and water is produced at the cathode. Therefore, with the electrode potential fixed, the local. current density and flux of water change. Here we account for these variations in the $z$ direction.

Table 2 .

Inlet gas composition.

\begin{tabular}{|c|c|c|}
\hline \multicolumn{2}{|c|}{ cathode-gas } & anode-gas \\
\hline $\begin{array}{l}\mathrm{x}_{\mathrm{H}_{2} \mathrm{O}} \\
\mathrm{x}_{\mathrm{N}_{2}}^{\mathrm{N}_{2}} \\
\mathrm{x}_{2}\end{array}$ & $\begin{array}{l}0.36 \\
0.512 \\
0.128\end{array}$ & \begin{tabular}{l|l}
$\mathrm{x}_{\mathrm{H}_{2} \mathrm{O}}$ & 0.36 \\
$\mathrm{x}_{\mathrm{CO}_{2}}$ & 0.16 \\
$\mathrm{x}_{2} \mathrm{H}_{2}$ & 0.48
\end{tabular} \\
\hline parameter & value & \\
\hline $\begin{array}{c}T \\
L \\
p \\
\epsilon \\
\epsilon \\
g \\
F_{a}^{O}\end{array}$ & $\begin{array}{c}353.0 \mathrm{~K} \\
0.0175 \mathrm{~cm} \\
1.0 \mathrm{bar} \\
0.20 \\
0.40 \\
1.0 \times 10^{-2} \mathrm{~mol} / \mathrm{m} \cdot \mathrm{s}\end{array}$ & \\
\hline
\end{tabular}


For the conditions given in table 2, figure 3 shows a twodimensional water map in the nembrane. $y$ is the dinension across the separator, and $z$ is along the length of the assembly. With increasing $z$, the partial pressure of water increases in the channel because of the overall chemical reaction and because the only means of water removal is in the gas sireams. Consequently, the level. of hydration in the membrane increases with larger $z$. As before, there is a concentration gradient establisher. that counteracts the electroosmotic drag of water and reduces the net transport of water across the cell.

The mole fractions of hydrogen, oxygen, and water as a function of axial position are shown in figure 4. The local current density is also depicted by the solid line in figure 4. At small $z$ there is a large net flux of water from the anode to the cathode gas stream. Consequently, the mole fraction of hydrogen is nearly constant for the first couple of centimeters. Initially the current density decreases; but as the membrane becomes more hydrated, the conductivity increases substantially and the local current density rises.

Figure 5 shows the composition of water in the two gas streams. We have introduced the variables

$$
x_{i}=\frac{F_{i}}{F_{\text {inert }}}=\frac{x_{j}}{x_{\text {inert }}}
$$

where inert refers to the nonreacting gas in each stream: nitrogen for the cathode and carbon dioxide for the anode; and $f$ is the molar flow rate of the cathode gas stream divided by the stoichiometric 


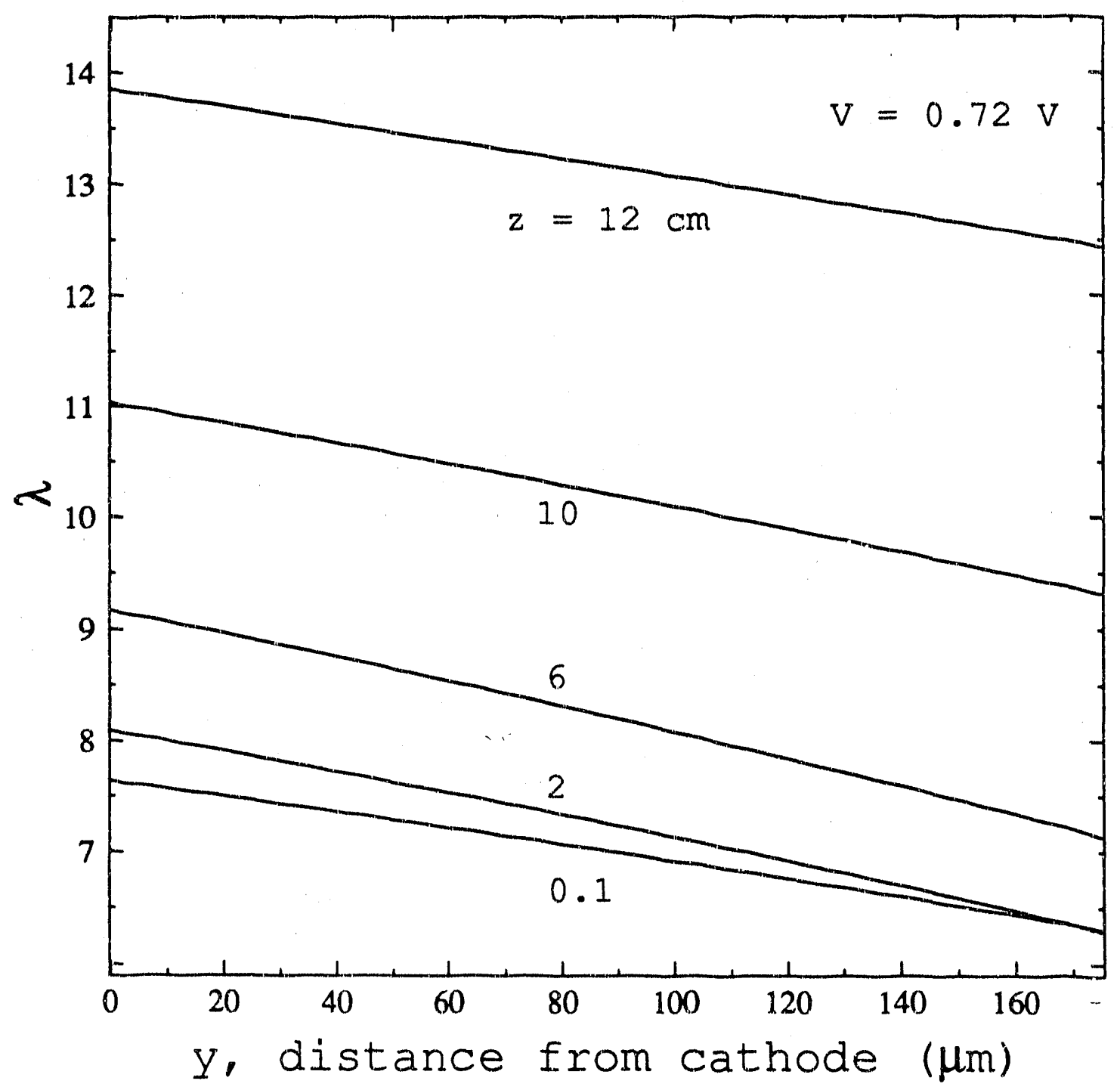

Figure 3. Concentration profiles of water across membrane. $\lambda$ is the number of water molecules per sulfonic acid group. $V=0.72$ volts, and $z$, the distance from the top of the channel, is a parameter. 


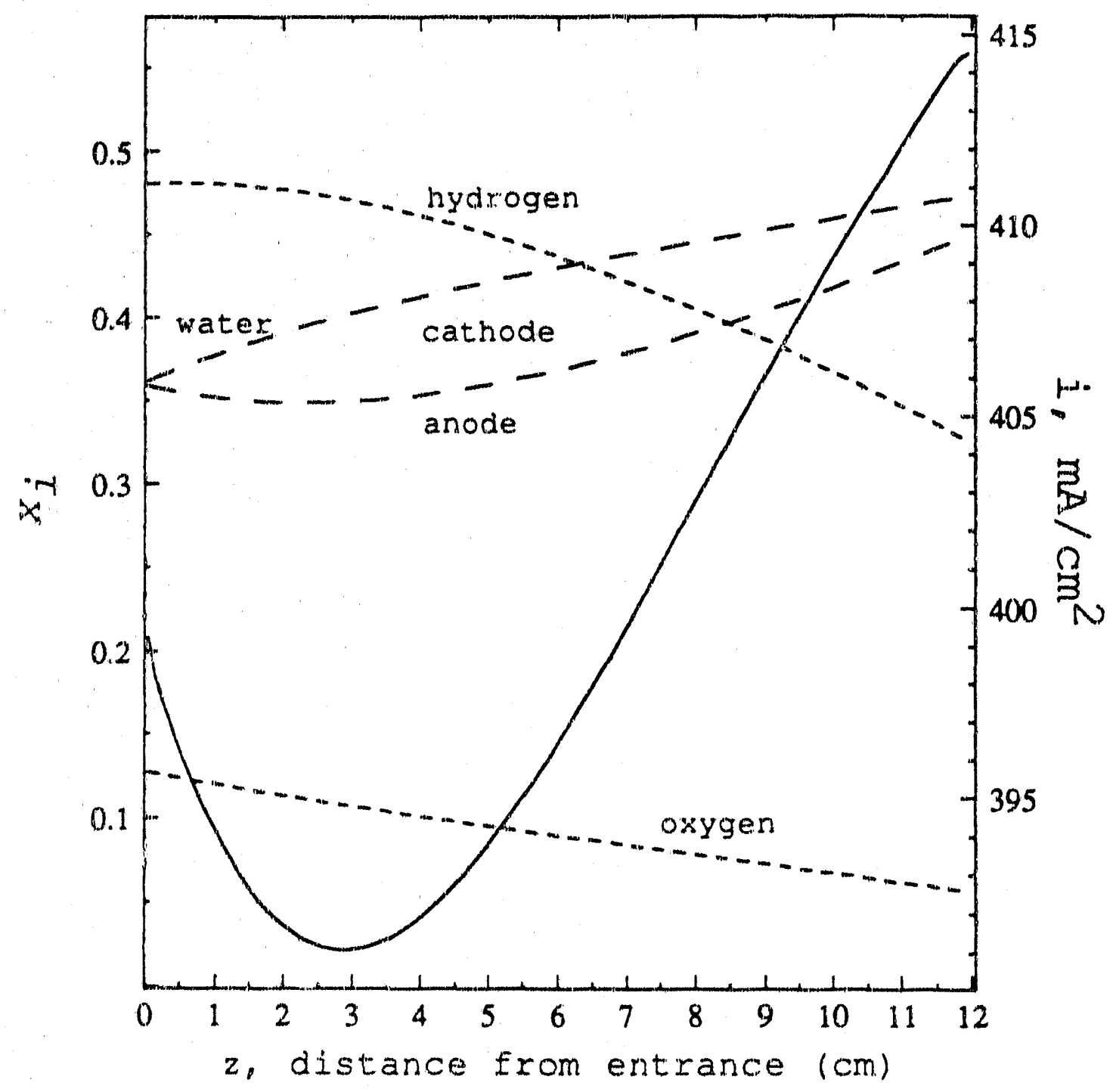

Figure 4. Mole fraction of hydrogen, oxygen, and water in the gas channels. Local current density is shown by the solid line. $V=0.72$ volts, and $f=1.0$. 


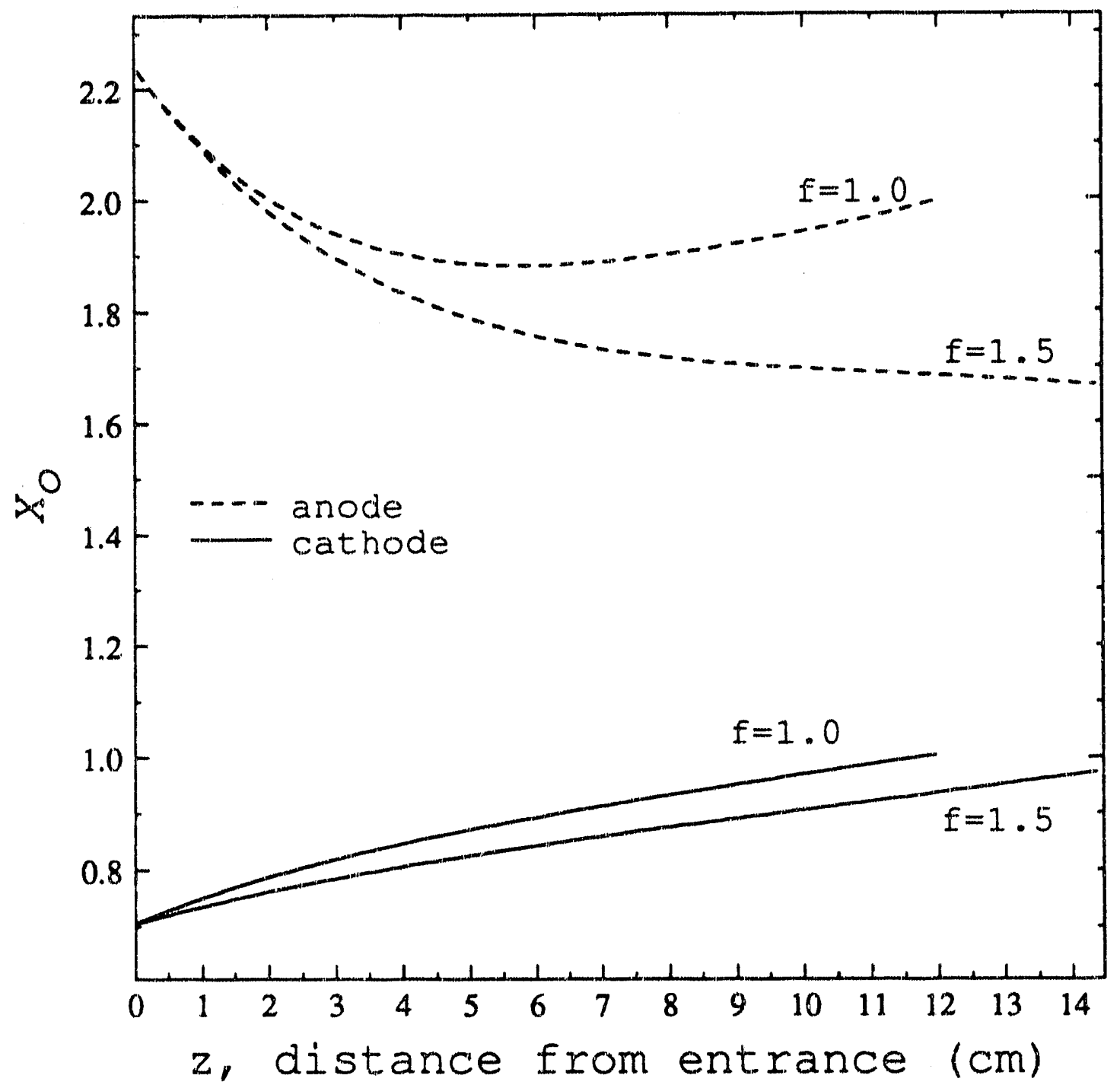

Eigure 5. Composition of water in gas channels. $f$ is the air-to-fuel ratio. Simulation is stopped at the value of $z$ where the cathode gas stream reaches saturation with respect to water. Utilization of hydrogen is than 0.52 and 0.67 for $f=1$ and 1.5 , respectively. 
flow rate for a specified anode gas flow rate. The mole ratio of water in the cathode stream increases; and for $f=1.5$, the mole ratio in the anode stream decreases. For $f=1$, the composition of water in the anode stream goes through a minimum; at which point water is being removed from both sides of the assembly. Figure 7 makes this clearer.

As the flow rate of air is increased, one observes that: 1) the cell is able to operate longer before the cathode stream becomes saturated, and 2) more water is removed in cathode gas stream, and the anode is further dehydxated. Thus, increasing air flow doesn't impact negatively on hydrugen utilization. The simulation was stopped when the cathode stream became saturated with respect to water. For $f=1$ and $f-1.5$, this point corresponds to hydrogen utilization of 0.52 and 0.67 , respectively.

In figure 6, the local superficial current density is plotted against local hydrogen utilization, with the cell potential as a parameter. The fractional utilization of hydrogen,

$$
u=\frac{x_{H_{2}}^{\circ}-X_{H_{2}}}{x_{H_{2}}^{\circ}}
$$

is an important parameter in the optimization of a fuel cell [3]. With the potential of the two electrodes constant, one expects the current density to decrease as the reactants are consumed. In this case, however, the conductivity increased dramatically with hydration 


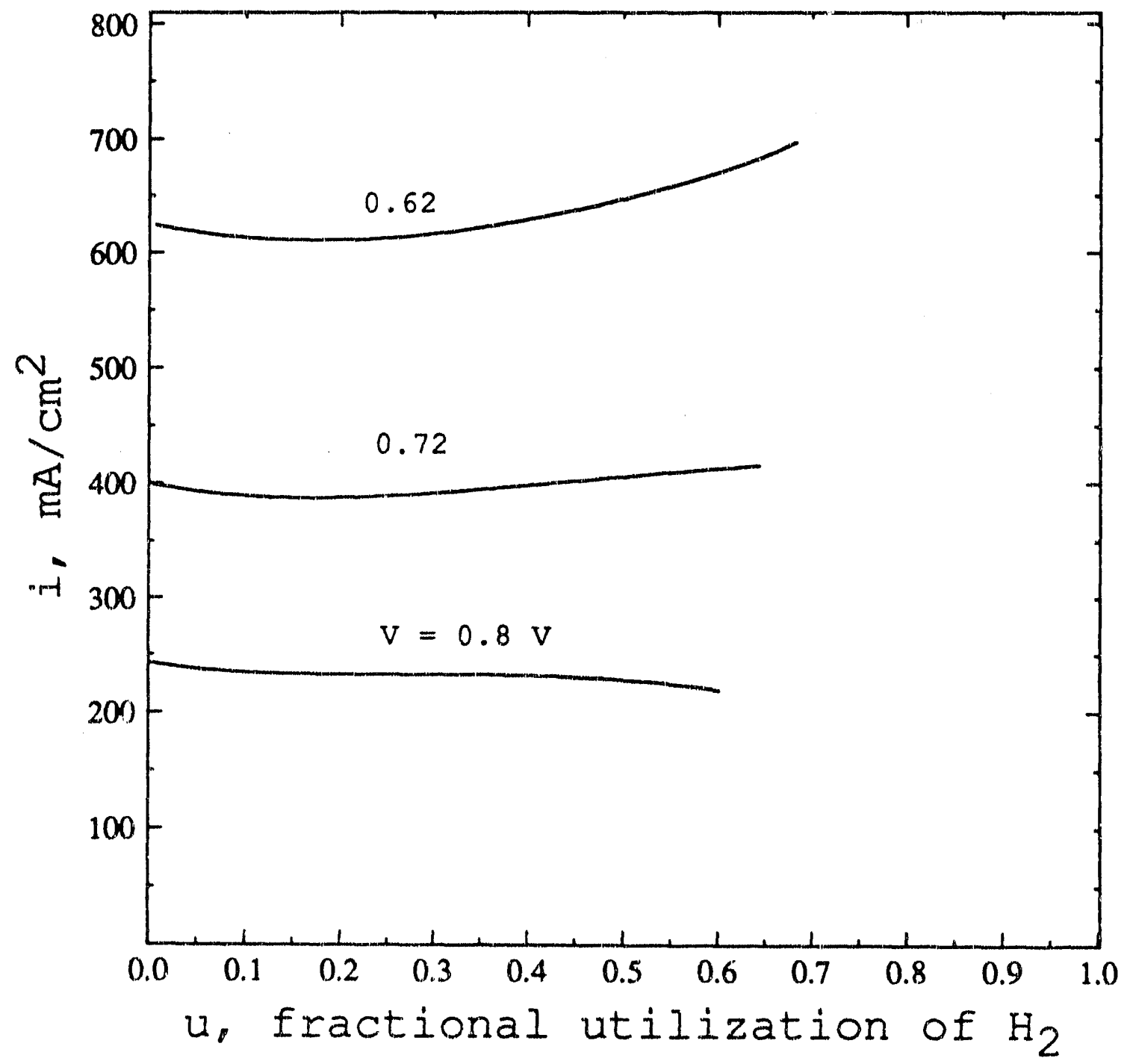

Figure 6. Jocal current density as a function of local utilization of hydrogen with $V$, the cell potential, as a parameter. $f=1.5$. 
of the membrane, and we observe an improvement in performance. At higher current densities, ohmic losses are more important, and consequently the improvement in performance is more dramatic. Because our model does not consider the condensation of water, we stop the simulation when the partial pressure of water equals the vapor pressure of the liquid, and we don't observe the utilization going to unity.

The net transport of water across the separator is shown in figure 7. At the top of the channel $(z-0)$, the partial pressure of water is identical in the two gas channels, and consequently there is a large flux from the anode to the cathode due to the electroosmotic drag of water. Farther down the channel, the partial pressure of water in the cathode stream becomes greater than that of the anode, and the current density diminishes, thus lessening the net flux of water. With a large enough difference in partial pressure of water across the membrane, the flow of water can be reversed. This is seen in figure 7 at $z \approx 6 \mathrm{~cm}$ for $f=1$. Here water is being removed from both sides.

Finally, we examine the heat transfer required to maintain constant temperature. There are significant losses and generation of heat. From the first law of thermodynamics,

$$
\Delta H=Q-W
$$

The work is given by 


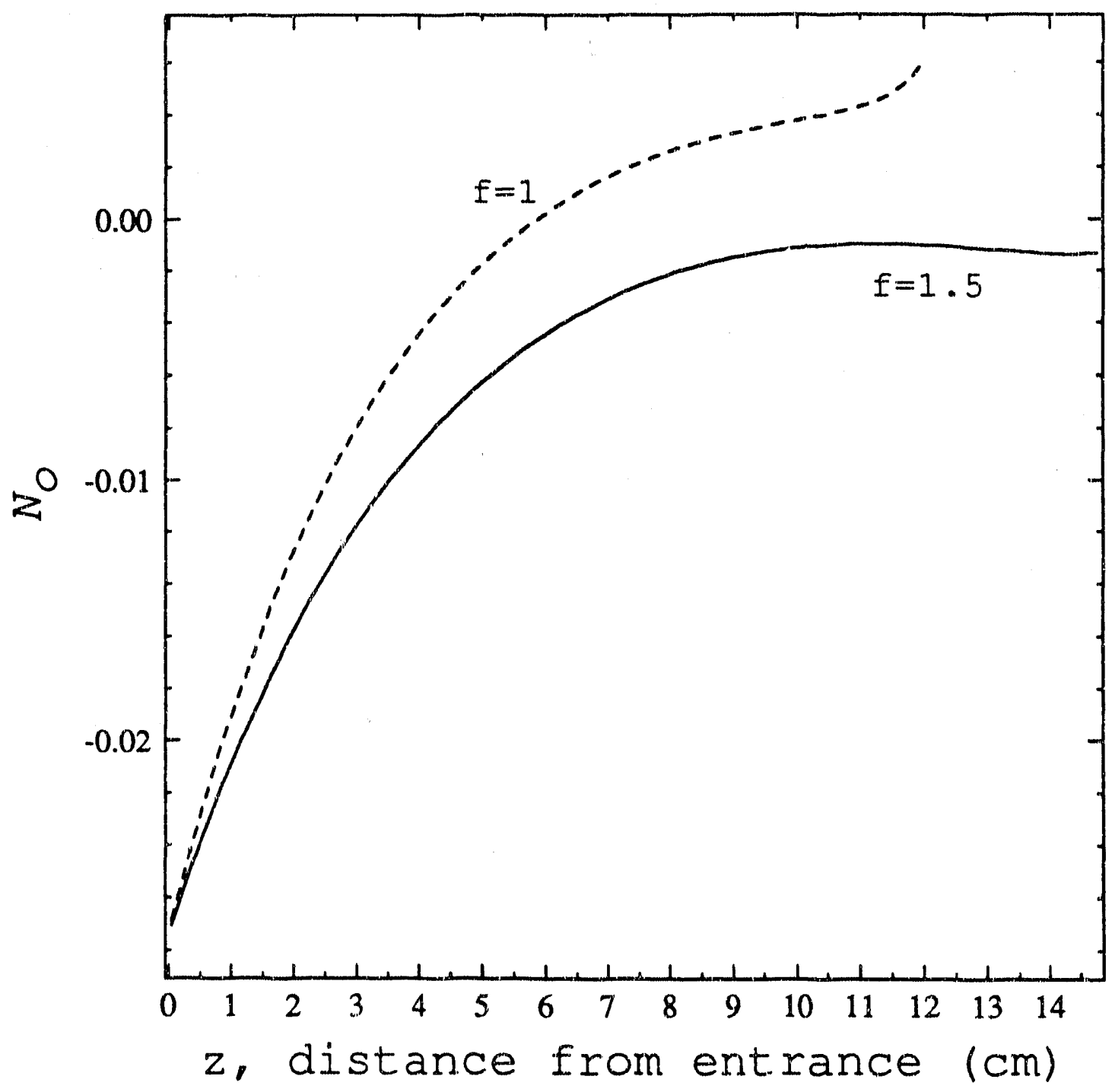

Eigure 7. Net flux of water across membrane. A value of the flux less than zero indicates transport from the anode to the cathode. $f$ is the air-to-fuel ratio. Simulation is stopped at the value of $z$ where the cathode gas stream reaches saturation with respect to water. 


$$
W-V \int i(z) d z \text {. }
$$

With $\mathrm{fu} I, V=0.72 \mathrm{~V}$, and for the conditions of table 2 after $10 \mathrm{~cm}$, the following is calculated:

\begin{tabular}{|c|c|}
\hline parametex & value \\
\hline$\Delta H$ & $-0.5147 \mathrm{~kW} / \mathrm{m}$ \\
$W$ & 0.3208 \\
$Q$ & -0.1939 \\
\hline
\end{tabular}

From the heat transfer required we can estimate the necessary heat transfer coefficient, $h$.

$$
h=\frac{-Q}{L \Delta T}
$$

Substitution of the values in the table above with ambient temperature of $298 \mathrm{~K} \quad(\Delta T-55 \mathrm{~K})$ gives a heat-transfer coefficient of $h=35.3 \mathrm{~W} / \mathrm{m}^{2} \cdot \mathrm{K}$. This is a typical value for forced convection of gases [4].

From this analysis we are able to draw some conclusions. First, substantial heat removal is required to maintain the system at constant temperature. The temperature difference we used is relatively large, and it seems impractical to increase this substantially. Thus at higher current densities, larger heat transfer coefficients are needed. Second, there is significant transport of water from the anode to the cathode. Although our model is not able to simulate countercurrent flow of fuel and air streams, our results indicate that this could be helpful. 


\subsection{Nonisothermal Case}

Temperature has a profound effect on the equilibrium and kinetic properties. In this last section, we assume that there are no temperature variations across the assembly (the $y$ direction) and that there is no transfer of heat in the $z$ direction. The temperature of the gases at the inlet is specified, and we calculate the temperature in the $z$ direction based on an overall energy balance. Heat is carried out by both an increase in temperature of the flowing gases and by heat transfer to some other medium at a temperature of $298 \mathrm{~K}$.

Figure 8 shows water profiles across the membrane with $z$ as a parameter. The operating conditions are shown in table 3 and include a heat-transfer coefficient of $30 \mathrm{~W} / \mathrm{m}^{2} \cdot \mathrm{K}$. The current density as a function of local utilization of hydrogen is shown in figure 9 ; the corresponding temperature profiles are depicted in figure 10.

Table 3 .

Inlet gas compositions.

\begin{tabular}{|c|c|c|c|}
\hline \multicolumn{2}{|c|}{ cathode-gas } & \multicolumn{2}{|c|}{ anode-gas } \\
\hline $\mathrm{x}_{H_{2}} \mathrm{O}$ & 0.40 & $\mathrm{x}_{H_{2}} \mathrm{O}$ & 0.40 \\
$\mathrm{x}_{N_{2}}$ & 0.48 & $\mathrm{x}_{\mathrm{CO}_{2}}$ & 0.15 \\
$\mathrm{x}_{\mathrm{O}_{2}}$ & 0.12 & $\mathrm{x}_{H_{2}}$ & 0.45 \\
& & ${ }_{2}$ & \\
\hline
\end{tabular}

\begin{tabular}{|c|c|}
\hline parameter & value \\
\hline$T$ & $353.0 \mathrm{~K}$ \\
$L$ & $0.0175 \mathrm{~cm}$ \\
$p$ & $1.0 \mathrm{bar}$ \\
$\epsilon \mathrm{m}$ & 0.20 \\
$\epsilon_{g}$ & 0.40 \\
$F^{\circ}$ & $1.0 \times 10^{-2} \mathrm{~mol} / \mathrm{m} \cdot \mathrm{s}$ \\
\hline
\end{tabular}




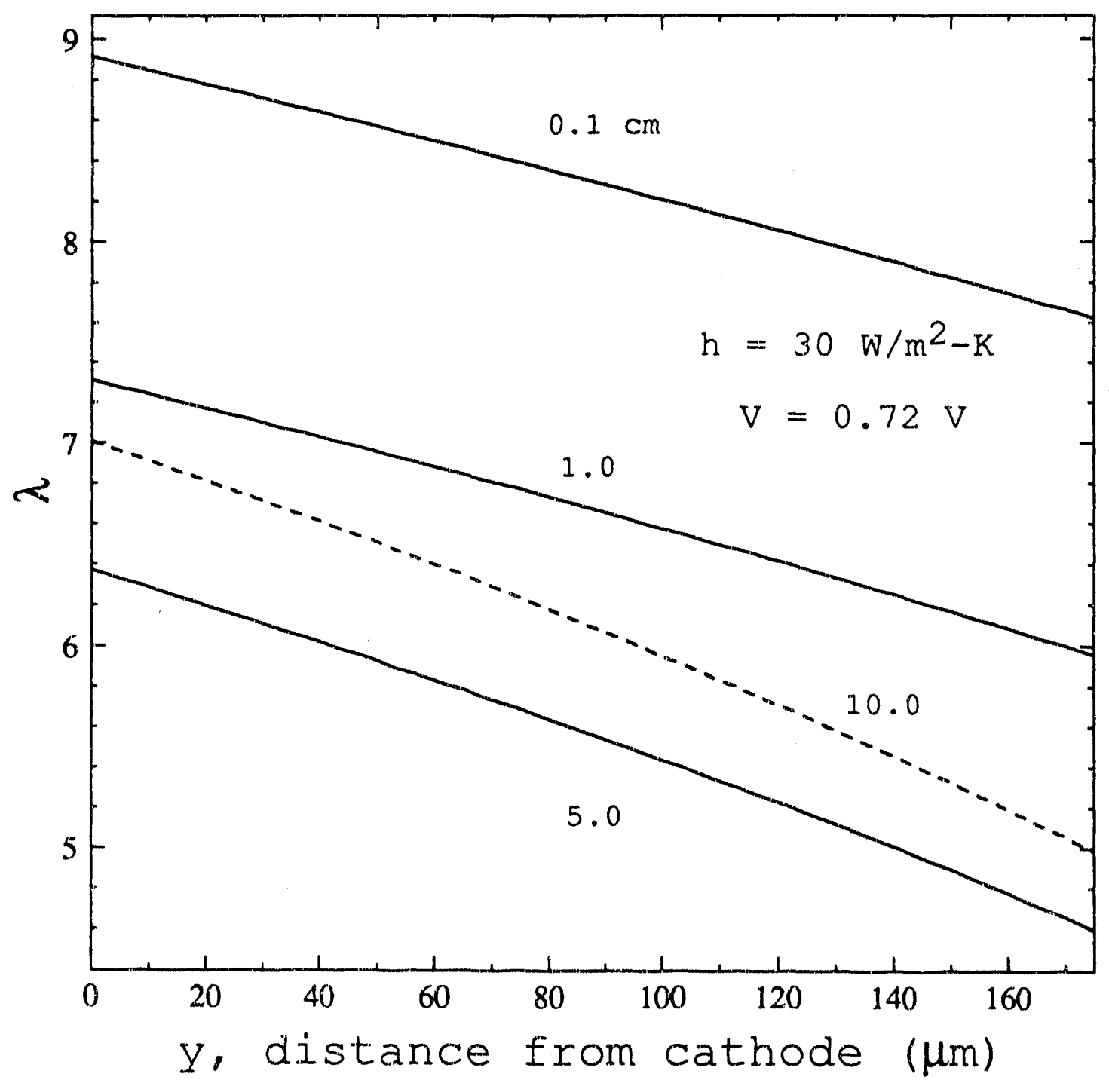

Figure 7. Concentration profiles of water across the membrane. $\lambda$ is the number of water molecules per sulfonic acid group. $V=0.72$ volts, and $z$, the distance from the top of the channel, is a parameter. 


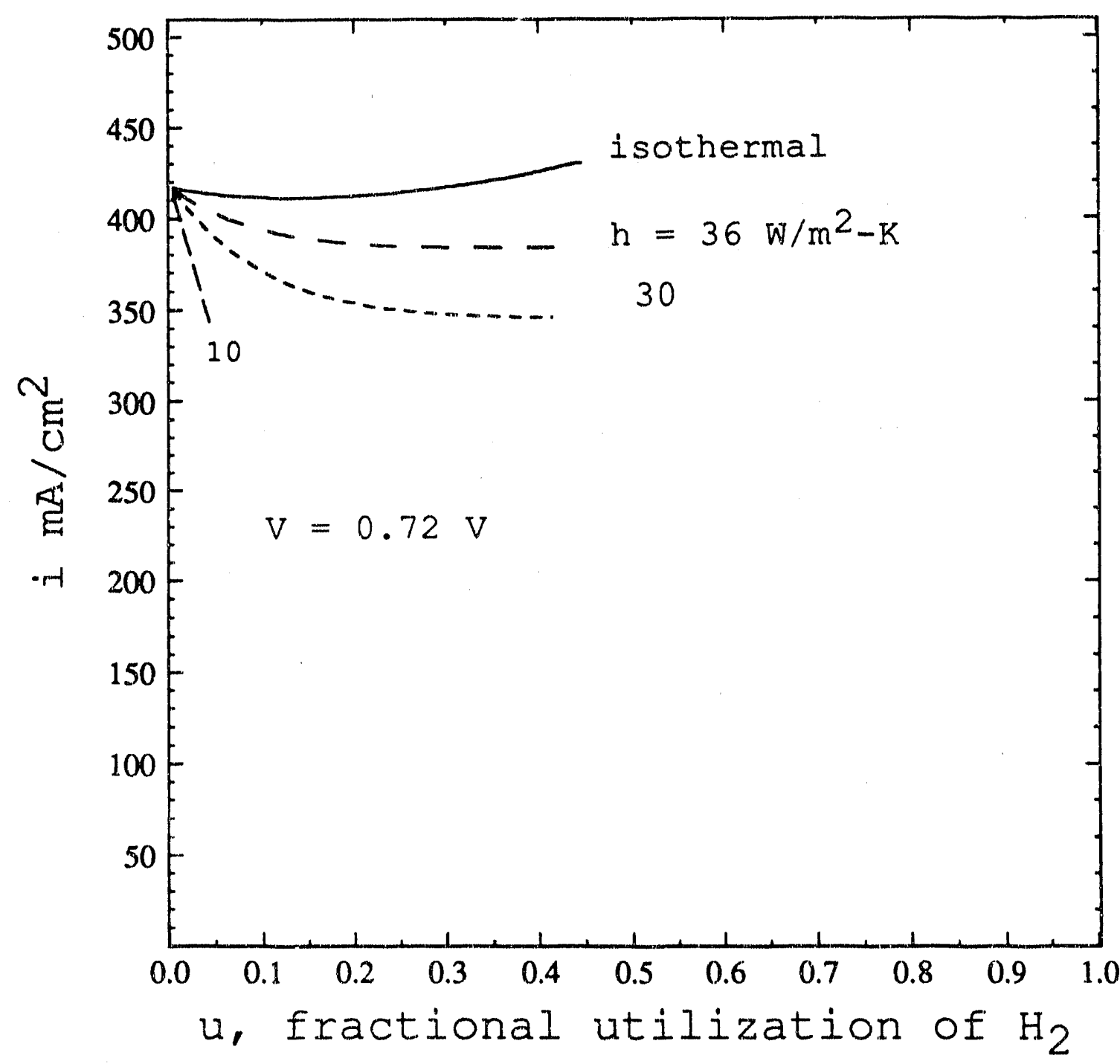

Figure 9. Local current density as a function of local utilization of hydrogen with $V$, the cell potential fixed, and the heat-transfer coefficient as a parameter. $f=1$. 


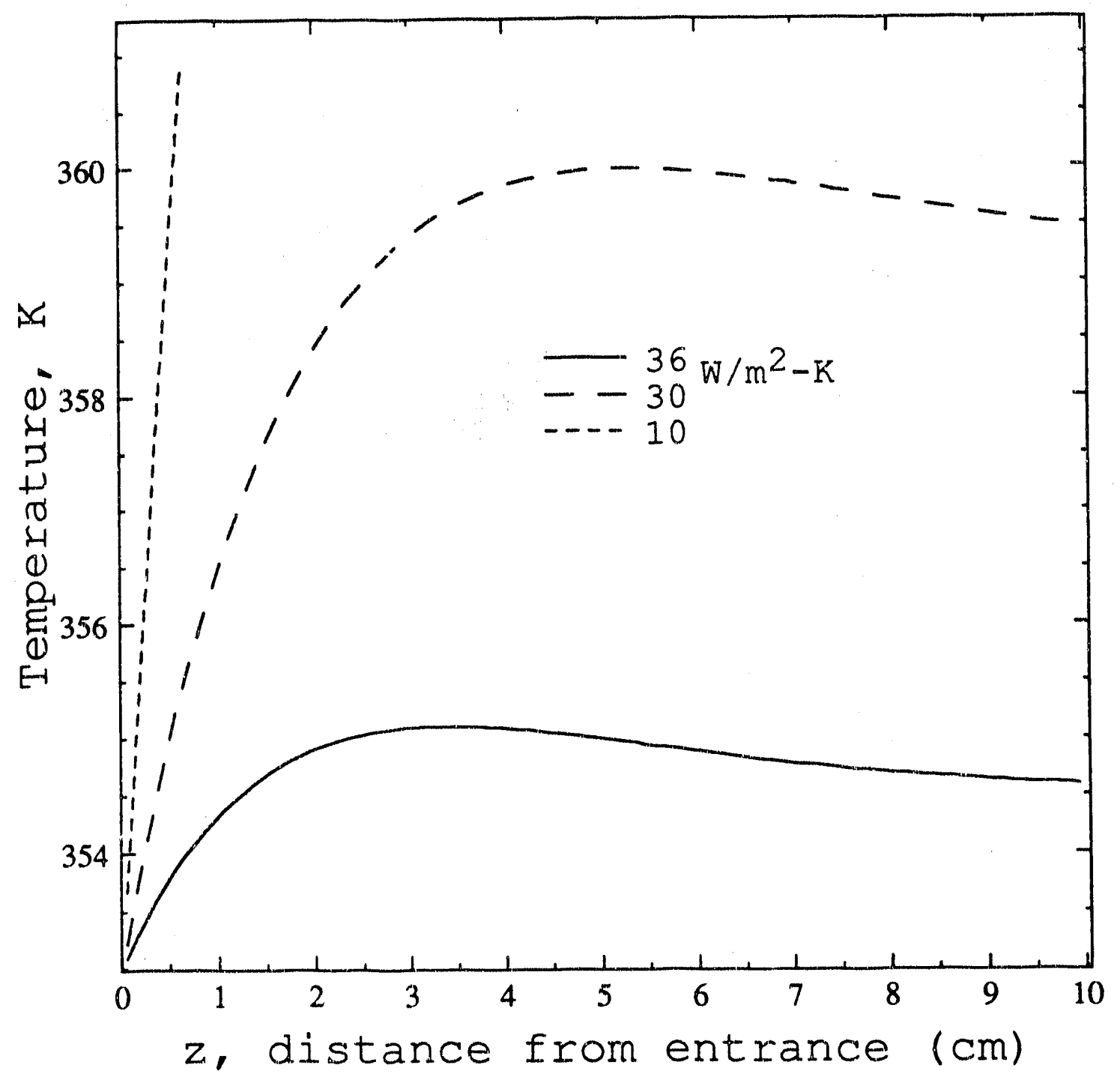

Figure 10. Temperature in gas channels. $f=1$ and $V=0.72 \mathrm{~V}$. 
The hydration of the membrane is sensitive to the rate of heat removal. Because of the low thermal capacity of the gas streams, the steady-state temperature will rise quickly without adequate removal of heat. At even moderated current densities, the cell would dehydrate, and the performance would be poor.

In contrast to the isothermal water profiles of figure 3 , for $h=30 \mathrm{~W} / \mathrm{m}^{2} \cdot \mathrm{K}$, initially the temperature rises sharply with increasing axial distance, and the merabrane becomes dehydrated (see figure 8). At constant cell potential, the loss of water from the membrane (and hence lower conductivity) results in lower current densities and the temperature levels off. Farther down the channel, the temperature decreases slowly, and the water conterit in the membrane increases (see the dashed line in figure 8 ).

The effect of the heat transfer coefficient on overall performance is seen in figure 9. For $h=36 \mathrm{~W} / \mathrm{m}^{2} \cdot \mathrm{K}$, the cell temperature rises slowly, and the performance is good. With a slightly lower value of the heat transfer coefficient $\left(h=30 \mathrm{~W} / \mathrm{m}^{2} \cdot \mathrm{K}\right)$, the temperature increases more sharply, the membrane dehydrates, and the performance decreases substantially. With little or no removal of heat $\left(h-10 \mathrm{~W} / \mathrm{m}^{2} \cdot \mathrm{K}\right)$, the temperature rises more than $10^{\circ} \mathrm{C}$ in $1 \mathrm{~cm}$, and there is a dramatic drop in the local current density.

From this we conclude that thermal considerations must be included in an analysis of water management. Thus, thermal control is critical to the performance of the solid-polymer-electrolyte fuel cell. Furthermore, the heat-transfer requirements will change 
markedly with current density, thus making temperature control more difficult.

It has been observed that the performance of these cells improves when supersaturated gas streams are used. This may le not so much because of the additional water supplied to the anode, but rather, because of improved heat transfer (dehydration of the membrane was not seen in the isothermal case). The evaporation of liquid water would result in a high heat-transfer coefficient, providing good temperature stability.

\section{List of Symbols}

$\begin{array}{ll}f & \text { air to fuel ratio } \\ F_{i} & \text { molar flow rate in } z \text { direction, } \mathrm{mol} / \mathrm{s} \cdot \mathrm{m} \\ h & \text { heat transfer coefficient, } \mathrm{W} / \mathrm{m}^{2} \cdot \mathrm{K} \\ \Delta H & \text { enthalpy, } \mathrm{J} / \mathrm{s} \cdot \mathrm{m} \\ i & \text { current density, A/cm } \\ L & \text { width of membrane, cm } \\ N_{i} & \text { molar flux in y direction, } \mathrm{mol} / \mathrm{cm}^{2} \cdot \mathrm{s} \\ P & \text { pressure, bar } \\ Q & \text { rate of heat transfer, } \mathrm{W} / \mathrm{m} \\ R & \text { universal gas constant, } 8.3143 \mathrm{~J} / \mathrm{mol} \cdot \mathrm{K} \\ T & \text { temperature, } \mathrm{K} \\ u & \text { utilization of hydrogen } \\ U & \text { open-circuit cell potential, } \mathrm{V}\end{array}$




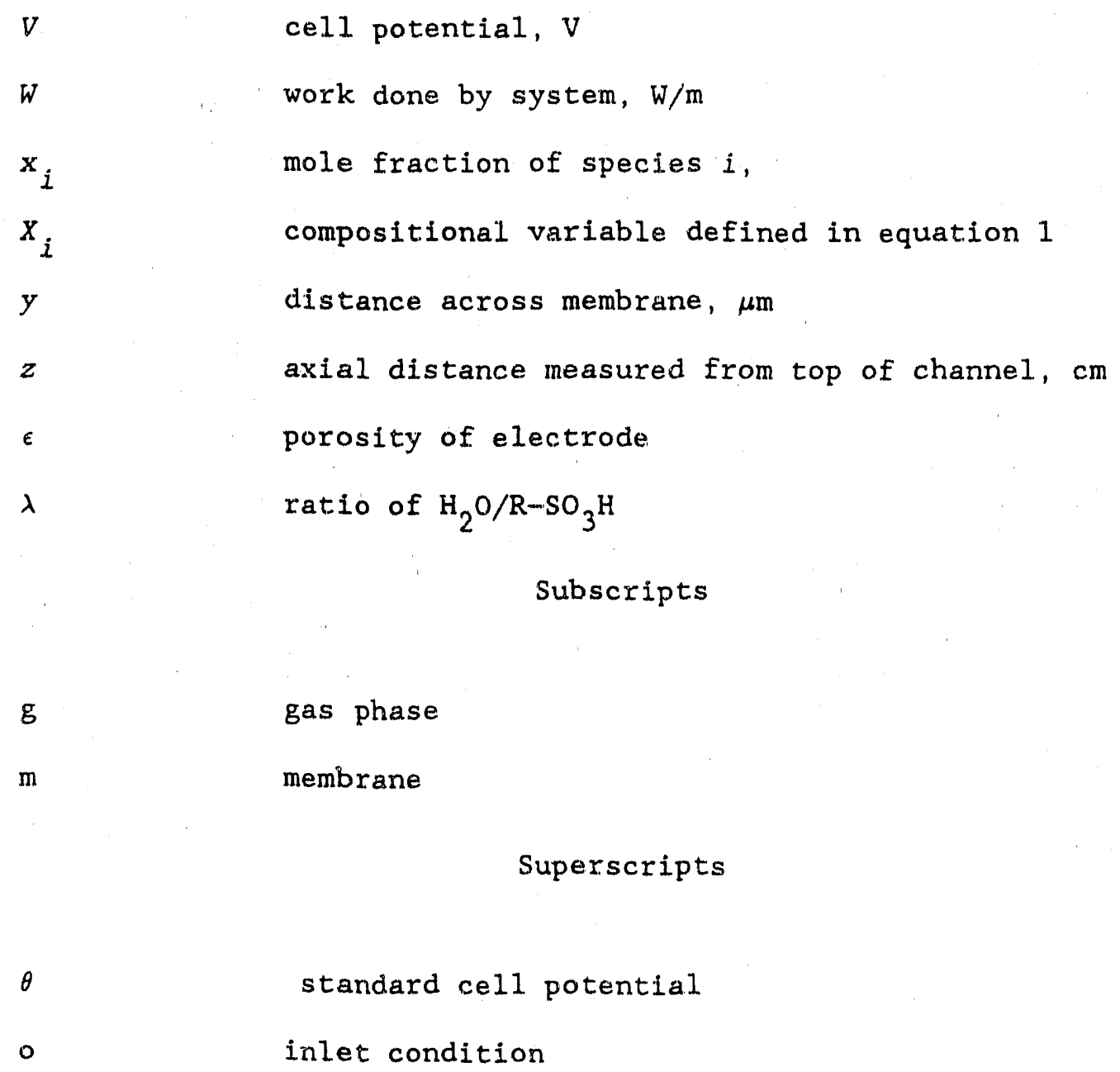

\section{References}

[1] T. Nguyen, J. C. Hedstrom, and N. E. Vanderborgh, "Heat and Mass Transfer Design Issues in PEM Fuel Cell Hardware," Proceedings of the Symposium on Fuel Celis, edited by R. E. White and A. J. Appleby, proceedings volume 8-14, The Electrochemical Society, Pennington, N. J. (1989). 
(2) E. A. Ticianelli, C. R. Derouin, A. Redondo, and S. Srinivasan, "Methods to Advance Technology of Proton Exchango Mernbrane Fuel. Cells," J. Electrochem. Soc., 135, 2209-2214 (1988).

[3] John Newnan, "Optimization of Potential and Hydrogen Utilization in an Acid Fuel Cell," Electrochim. Acta, 24, 223-229 (1979).

[4] F. P. Incropera and D. P. Dewitt, Fundamentals of heat Transfer, John Wiley \& Sons, New York (1981). 
Appendix $\mathrm{B}$

Physicochemical properties

\section{Transport properties}

The transport properties of the polymer electrolyte were discussed in chapter 5. The diffusion coefficients in the gas phase were estimated from kinetic theory. Assuming the ideal-gas law holds, Bird et al. [1] gave the approximate formula

$$
D_{A B}=1.8583 \times 10^{-5} \frac{\sqrt{T^{3}\left(\frac{1}{M_{A}}+\frac{1}{M_{B}}\right)}}{p \sigma_{A B}^{2}{ }_{A B}},
$$

where $\Omega_{A B}$ is a tabulated function of temperature for a Lennard-Jones gas. The parameters of the equation B-I were taken from Reid et al. [2] and are shown in table B-1. We assumed

$$
\epsilon_{A B}=\left(\epsilon_{A} \epsilon_{B}\right)^{\frac{1}{2}}
$$

and

Table B-1.

\begin{tabular}{|c|c|r|r|}
\hline species & $M_{i}, \mathrm{~g} / \mathrm{mol}$ & $\epsilon / \mathrm{k}, \mathrm{K}$ & $\sigma, \mathrm{nm}$ \\
\hline $\mathrm{H}_{2} \mathrm{O}$ & 18.02 & 809.1 & 0.2641 \\
$\mathrm{~N}_{2}^{2}$ & 28.01 & 71.4 & 0.3798 \\
$\mathrm{O}_{2}^{2}$ & 32.00 & 106.7 & 0.3467 \\
$\mathrm{CO}_{2}^{2}$ & 44.01 & 195.2 & 0.3941 \\
$\mathrm{H}_{2}$ & 2.016 & 59.7 & 0.2827 \\
\hline
\end{tabular}




$$
\sigma_{A B}=\frac{\sigma_{A}+\sigma_{B}}{2}
$$

We used Nuefeld's [3] relation to estimate $\Omega_{A B}$ :

$$
\Omega_{A B}=\frac{A}{T_{*}^{B}}+\frac{C}{\exp \left(D T_{*}\right)}+\frac{E}{\exp \left(F T_{*}\right)}+\frac{G}{\exp \left(H T_{*}\right)}
$$

where $T_{*}=k T / \epsilon_{A B}$. The parameters for equation $B-4$ are listed in table $B-2$.

The effective diffusion coefficient depends on the porosity of the material. However, experimental results are usually necessary to quantify this relationship. Meredith and Tobias [4], for example, studied the conductivity of porous media in detail. Because the porosity of the fuel-cell assembly is not well characterized, the relationship is somewhat arbitrary, and for convenience we chose

$$
D_{i j}^{e f f}=\epsilon D_{i j}
$$

This $\epsilon$, the porosity of the medium, is different from that in equation $B-4$ and table $B-1$.

Table B-2

\begin{tabular}{|l|l|l|}
\hline$A=1.06036$ & $D=0.47635$ & $G=1.76474$ \\
\hline$B=0.15610$ & $E=1.03587$ & $H=3.89411$ \\
\hline$C=0.19300$ & $F=1.52990^{\circ}$ & \\
\hline
\end{tabular}




\section{Thermodynamics}

Zawodzinski et al. [5] measured the absorption isotherm of Nafion 117 membrane at $30^{\circ} \mathrm{C}$.

$$
\begin{gathered}
\lambda=0.043+17.81 a_{0}-39.85 a_{0}^{2}+36.0 a_{0}^{3} . \\
a_{0}=-0.0505+0.1853 \lambda-0.01049 \lambda^{2}+1.712 \times 10^{-4} \lambda^{3} .
\end{gathered}
$$

The activity of water is given by $p_{0} / p^{*}$. The vapor pressure of water is calculated from the Antoine's correlation

$$
\ln p^{*}-A-\frac{B}{T+C}
$$

The constants are from [2]: $A=11.6832, B-3816.44, C=-46.13 . p^{*}$ is the vapor pressure in bar.

The enthalpy of a gaseous species is

$$
\vec{H}_{i}=\tilde{H}_{i}-\tilde{H}_{i}^{*}\left(T_{0}\right)+\int_{T_{0}}^{T} C_{p} d T,
$$

and its heat capacity is

$$
\tilde{c}_{p}^{*}=a+b T+\frac{c}{x^{2}}
$$

The values of the constants for our fuel-cell system are taken from [6] and given in table B-3. 
Table B-3.

\begin{tabular}{|c|c|c|c|c|}
\hline species & $\tilde{H}_{i}^{*}, \frac{k J}{m o l}$ & $a, \mathrm{~J} / \mathrm{mol} \cdot \mathrm{K}$ & $10^{3} \mathrm{~b}, \mathrm{~J} / \mathrm{mol} \cdot \mathrm{K}^{2}$ & $10^{-5} \mathrm{c}, \mathrm{J} \cdot \mathrm{K} / \mathrm{mol}$ \\
\hline $\mathrm{H}_{2}{ }^{\mathrm{N}}$ & -241.82 & 30.54 & 10.29 & 0.0 \\
$\mathrm{~N}_{2}^{2}$ & 0.0 & 28.58 & 3.77 & -0.50 \\
$\mathrm{O}_{2}^{2}$ & 0.0 & 29.96 & 4.184 & -1.67 \\
$\mathrm{CO}_{2}$ & -393.51 & 44.22 & 8.79 & -8.62 \\
$\mathrm{H}_{2}$ & 0.0 & 27.28 & 3.26 & 0.50 \\
\hline
\end{tabular}

The hydrogen-oxygen fuel cell can be represented as

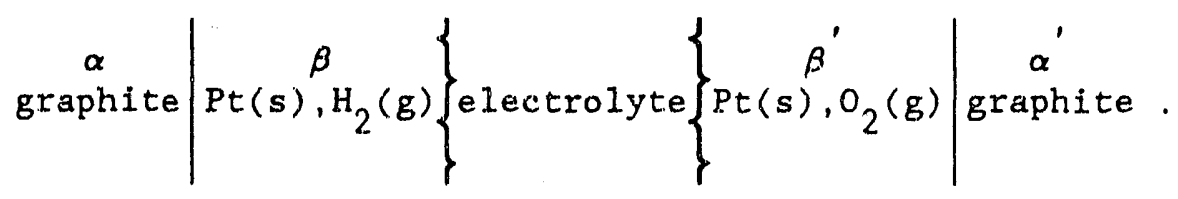

The potential of this cell is

$$
F U=-F\left(\Phi^{\alpha}-\Phi^{\alpha}\right)-\mu^{\alpha} e^{-}-\mu^{\alpha} e^{-\cdots}
$$

For the two half-cell reactions

$$
\mathrm{H}_{2} \rightleftarrows 2 \mathrm{H}^{+}+2 \mathrm{e}^{-}
$$

and

$$
2 \mathrm{H}_{2} \mathrm{O} \rightleftarrows \mathrm{O}_{2}+4 \mathrm{H}^{+}+4 \mathrm{e}^{-}
$$

the cell potential is

$$
F U=F U^{\theta}+h_{2} R T \ln p_{H_{2}}+\frac{1}{4} R T \ln p_{O_{2}}-h_{2} R T \ln a_{0}^{\beta^{\prime}}+\left(\mu_{+}^{\beta}{ }_{-} \beta_{+}^{\beta^{\prime}}\right) .
$$

The transport number of water is given in chapters 4 and 5 ; this is used to calculate the last term on the right side of equation B-12. 
At open circuit,

$$
\Delta \mu_{+}-\left(\mu_{+}^{\beta}-\mu_{+}^{\beta}\right)--\int\left(t_{0}^{m} / z_{0}\right) \operatorname{RTd}\left(\ln a_{0}\right)
$$

From the thermodynamic relation

$$
\frac{d(\Delta G / R T)}{d T}=-\frac{\Delta H}{R T^{2}}
$$

we can calculate the temperature dependence of the standard cell potential.

$$
U^{\theta}=\frac{T}{T_{0}} U_{0}^{\theta}+\frac{T}{2 F}\left\{-\Delta H\left(T_{0}\right)\left(\frac{1}{T}-\frac{1}{T_{0}}\right)+\Delta a\left(\ln \frac{T}{T_{0}}+\frac{T_{0}}{T}-1\right)+\frac{\left(T-T_{0}\right)^{2}}{2 T}\left(\Delta b+\frac{\Delta c}{T T_{0}^{2}}\right)\right\}
$$

\section{Kinetics}

Ticianelli et al. [7] and Uribe et al. [8] have studied the kinetics of oxygen reduction in solid-polymer-electrolyte fuel cells.

$$
2 \mathrm{H}_{2} \mathrm{O}-\mathrm{O}_{2}-4 \mathrm{H}^{+} \rightleftarrows 4 \mathrm{e}^{-} \text {. }
$$

The reaction is first order in oxygen concentration and the cathodic transfer coefficient is one. The exchange current density reported by Ticianelli is based on the superficial area of the electrodes. There is no clear way to differentiate between the kjnetic rate constants and the specific interfacial area. Therefore, we required that the open-circuit potential be consistent with our calculation 
above, and we adjusted $a$ and $k$ to approximate Ticianelli's currentvoltage curve.

Recently, Parthasarathy et al. [9] investigated the temperature dependence of the electrode kinetics of oxygen reduction.

$$
i_{0}=i_{0}\left(T_{r}\right) \exp \left[\frac{\Delta E}{R}\left[\frac{1}{T_{r}}-\frac{1}{T}\right)\right]
$$

We used the activation energy they reported $(\Delta E=73.2 \mathrm{~kJ} / \mathrm{mol})$ and variations in $U^{\theta}$ with temperature to account for the change in kinetic rate constants with temperature.

We assumed that the oxidation of hydrogen was fast and did not represent significant overpotential for the fuel cell. The absorption of water was also assumed to be fast.

$$
\mathrm{H}_{2} \mathrm{O}(\mathrm{l}) \rightleftarrows \mathrm{H}_{2} \mathrm{O}(\mathrm{g})
$$

The equilibrium constant for this reaction is calculated from the thermodynamic data presented above.

$$
K_{e q}=\frac{k_{f}}{k_{b}}=\frac{c_{o} R T}{P_{o}}
$$

$$
\text { List of Symbols }
$$

$\begin{array}{ll}a_{0} & \text { activity of water } \\ A & \text { constant in equation } B-8\end{array}$


B

$c_{i}$

C

$c_{p}$

$D_{i j}$

F

$G$

H

i。

K

$M_{i}$

$p$

$p_{i}$

$R$

$S$

$I$

$U$

6

$\lambda$

$\mu_{i}$

$\sigma_{A B}$

$\Phi$

$\Omega_{A B}$ constant in equation $B-8$

concentration of species i, mol/1

constant in equation $B-8$

molar heat capacity, $\mathrm{J} / \mathrm{mol} \cdot \mathrm{K}$

binary-interaction coefficient, $\mathrm{cm}^{2} / \mathrm{s}$

Faraday's constant $96,487 \mathrm{C} / \mathrm{eq}$

Gibbs energy, J

enthalpy, $\mathrm{J}$

exchange current density, $A / \mathrm{m}^{2}$

equilibrium constant

molar mass, $\mathrm{g} / \mathrm{mol}$

pressure, bar

partial pressure of species $i$, bar

universal gas constant, $8.3143 \mathrm{~J} / \mathrm{mol} \cdot \mathrm{K}$

entropy, $\mathrm{J} / \mathrm{K}$

temperature, $\mathrm{K}$

open-circuit cell potential, V

porosity

ratio of $\mathrm{H}_{2} \mathrm{O} / \mathrm{R}-\mathrm{SO}_{3} \mathrm{H}$

electrochemical potential of species $i, \mathrm{~J} / \mathrm{mol}$

characteristic length, nm

electrostatic potential, V

diffusion collision integral 


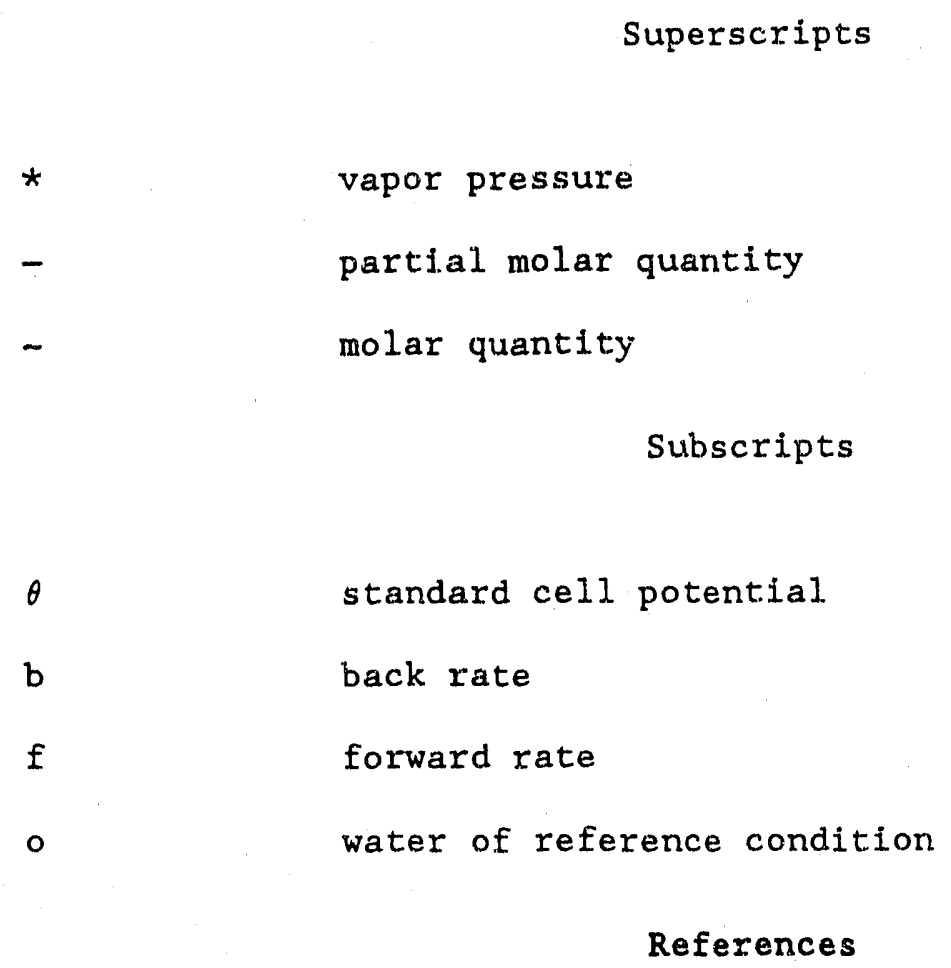

[1] R. Byron Bird, Warren E. Stewart, and Edwin N. Lightfoot, Transport Phenomena, John Wiley \& Sons, Inc., New York (1960).

[2] Robert C. Reid, John Prausnitz, and Thomas K. Sherwood, The Properties of Gases and Liquids, McGraw Hill Inc., New York (1977).

[3] P. D. Neufeld, A. R. Janzen, and R. A. Aziz, J. Chem. Phys., 57, 1100 (1972).

[4] R. E. Meredith and C. W. Tobias, "Conduction in Heterogeneous Systems," Advances in Electrochemistry and Electrochemical Engineering 2, C. W. Tobias, ed., Interscience Publishers, New York (1962). 
[5] T. Zawodzinski, M. Neeman, L. Sillerud, and S. Gottesfeld, "Determination of Water Diffusion Coefficients in Perfluorosulfonated Ionomeric Membranes," J. Phys. Chem., 95, 6040-6044 (1991).

[6] G. N. Lewis and M. Randall, revised by K. S. Pitzer and L. Brewer, Thermodynamics, McGraw-Hill Inc., New York (1961).

[7] E. A. Ticianelli, C. R. Derouin, A. Redondo, and S. Srinivasan, "Methods to Advance Technology of Proton Exchange Mem. brane Fuel Cells," J. Electrochem. Soc., 135, 2209-2214 (1.988).

[8] F. A. Uribe, T. E. Springer, and S. Gottesfeld, "A Microelectrode study of Oxygen Reduction at the Platinum Recast Nafion Film Interface," J. Electrochem. Soc., 139, 764-773 (1992).

[9] A. Parthasarathy, S. Srinivasan, A. J. Appleby, and C. R. Martin, "Temperature Dependence of the Electrode Kinetics of Oxygen Reduction at the Platinum/Nafion Interface - A Microelectrode Inves tigation," J. Electrochem. Soc., in press (1992). 


\section{Appendix C}

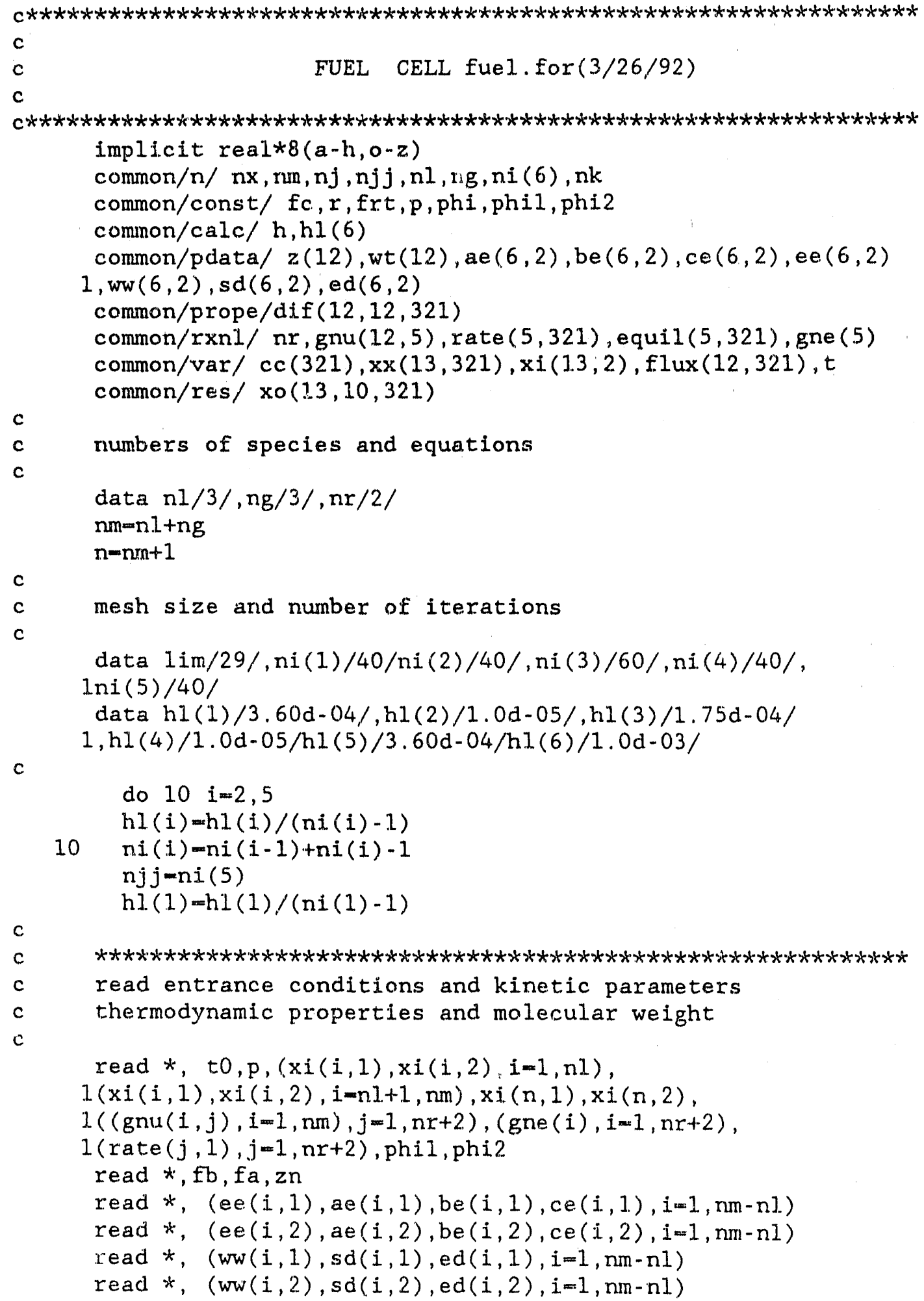




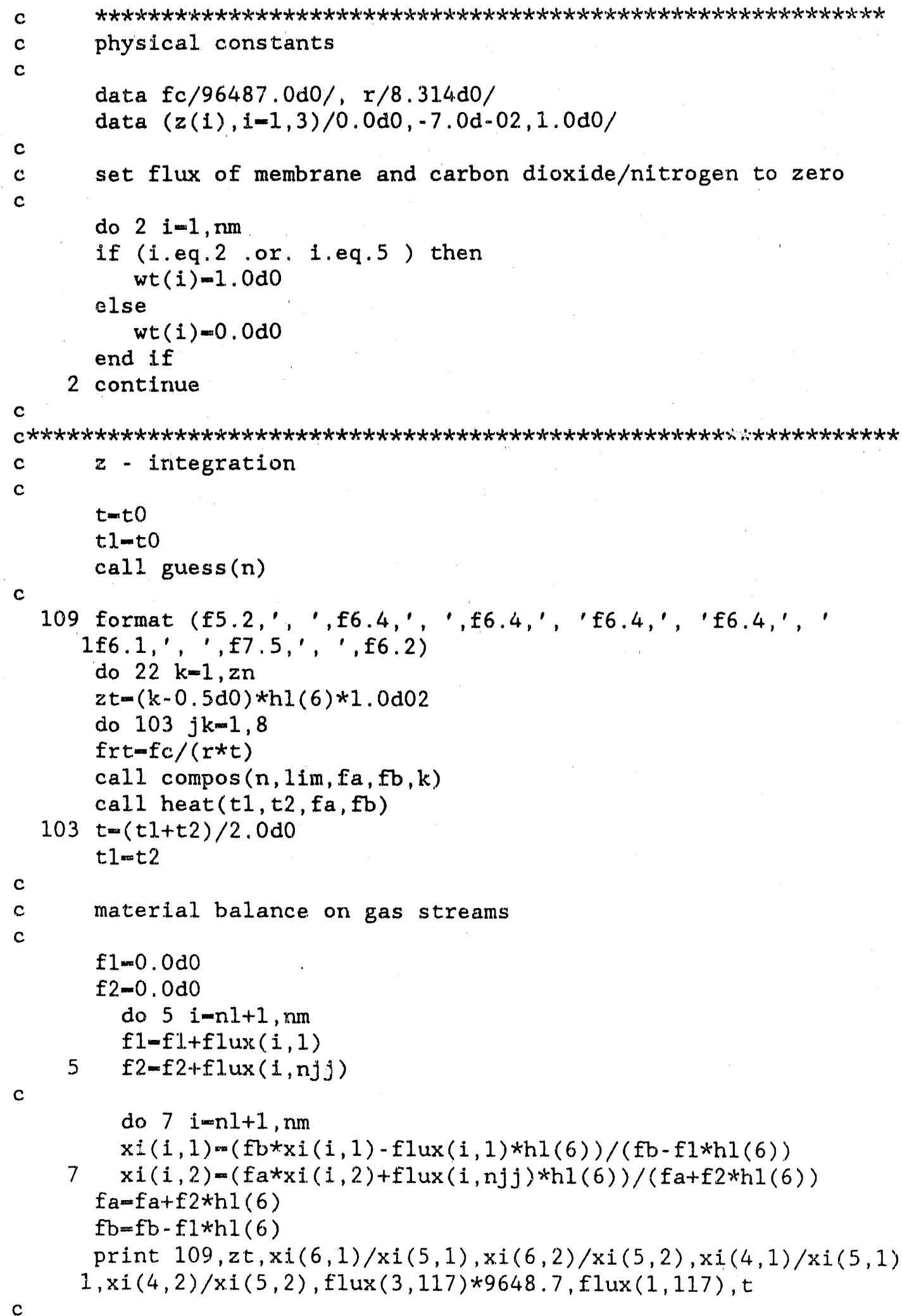


22 continue

$\mathrm{c}$

call nucamb $(n)$

$\mathrm{c}$

stop

end

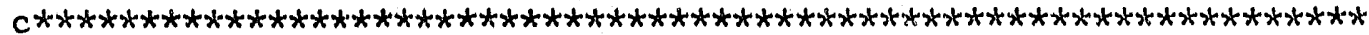

subroutine compos( $n, 1 i m, f a, f b, 1 i)$

implicit real*8(a-h,o-z)

common/n/ $n x, n m, n j, n j j, n l, n g, n i(6), n k$

common/const/ fc, $r, f r t, p, p h i, p h i l, p h i 2$

common/calc/ $\mathrm{h}, \mathrm{hl}(6)$

common/pdata/ $z(12)$, wt $(12)$, ae $(6,2)$, be $(6,2)$, ce $(6,2)$, ee $(6,2)$

$1, \operatorname{ww}(6 ; 2), \operatorname{sd}(6,2), \operatorname{ed}(6,2)$

common/prope/dif $(12,12,321)$

common/ssblock/ $\mathrm{xpO}(13), \mathrm{xx} 0(13,321)$

common/var/ $\operatorname{cc}(321), x \times(13,321), x i(13,2), f \operatorname{lux}(12,321), t$

common/comp/s1(13),s2(13),s3(26,26), s4(13), s5(13), binv $(13,13)$

common/mat/b, d

common/band/ a, c, g, x,y

dimension $b(26,26), d(26,53)$

dimension $a(26,26), c(26,321), g(26), x(26,26), y(26,26)$

dimension $\operatorname{xp}(26)$

c

99 format ( $1 \mathrm{~h}, / / 5 \mathrm{x}$, 'this run did not converge' $/ /$ )

$n x=n$

c

$n j=n j j$

do $20 j=1, n j$

do $20 \quad i=1, n$

c

20 $c(i, j)=x x(i, j)$

jcount $=0$

call prop (n,jcount, $1 \mathrm{i})$

$\mathrm{c}$

do $4 i=1, n$

4

$\operatorname{xp}(i)=0.0 \mathrm{~d} 0$

c

$c$

c

initialize variables to begin each iteration

(jcount is iteration \#)

$8 j=0$

jcount $=j$ count +1

do $9 i=1, n$

do $9 \mathrm{k}=1, \mathrm{n}$

$$
x(i, k)=0.0 \mathrm{~d} 0
$$

9

$$
y(i, k)=0.0 \mathrm{~d} 0
$$

store previous iteration of $(x p$ in $x p 0) \&(x x$ in $x x 0)$

do $6 i=1, n$

$\operatorname{xp} 0(i)=x p(i)$ 


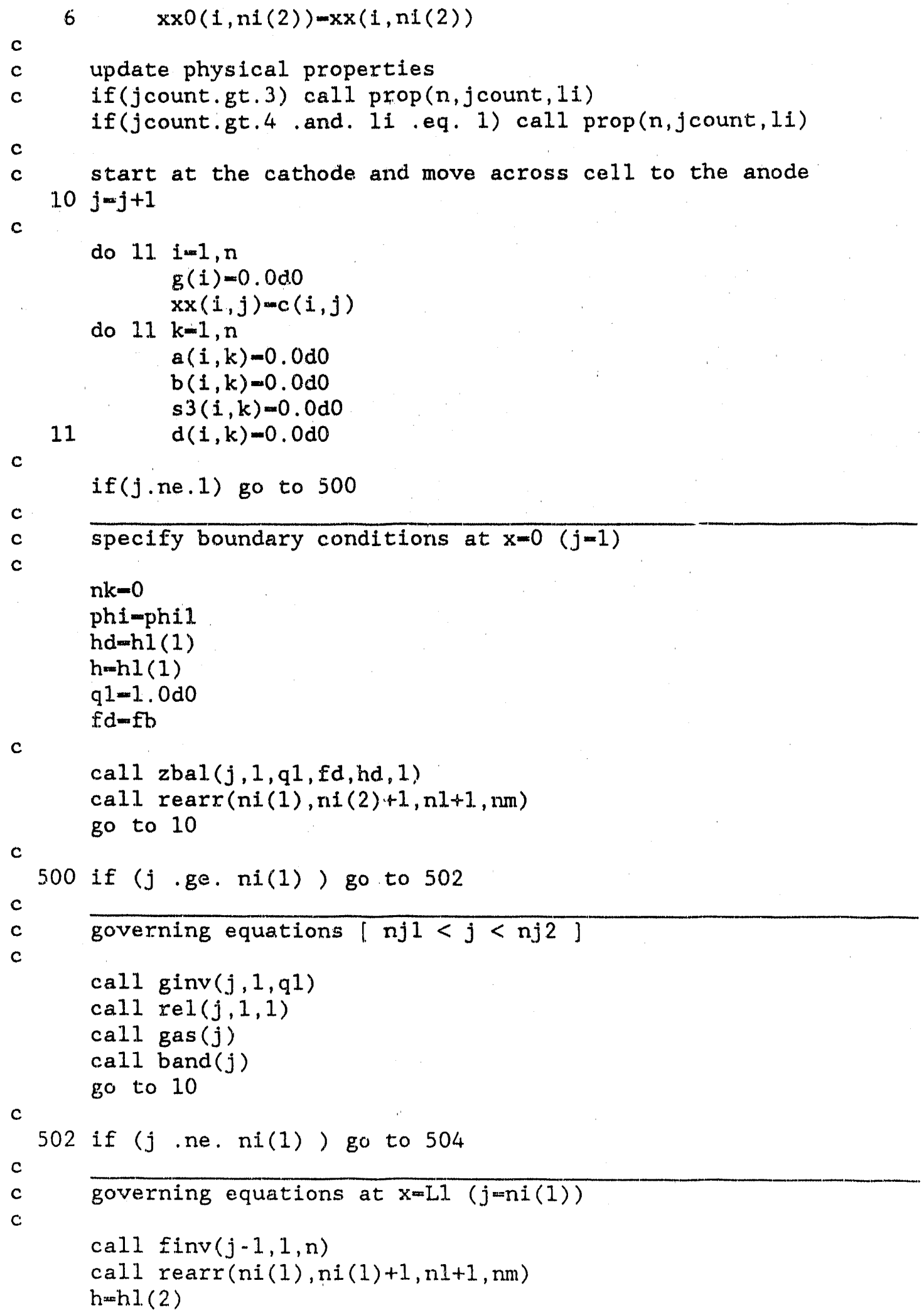




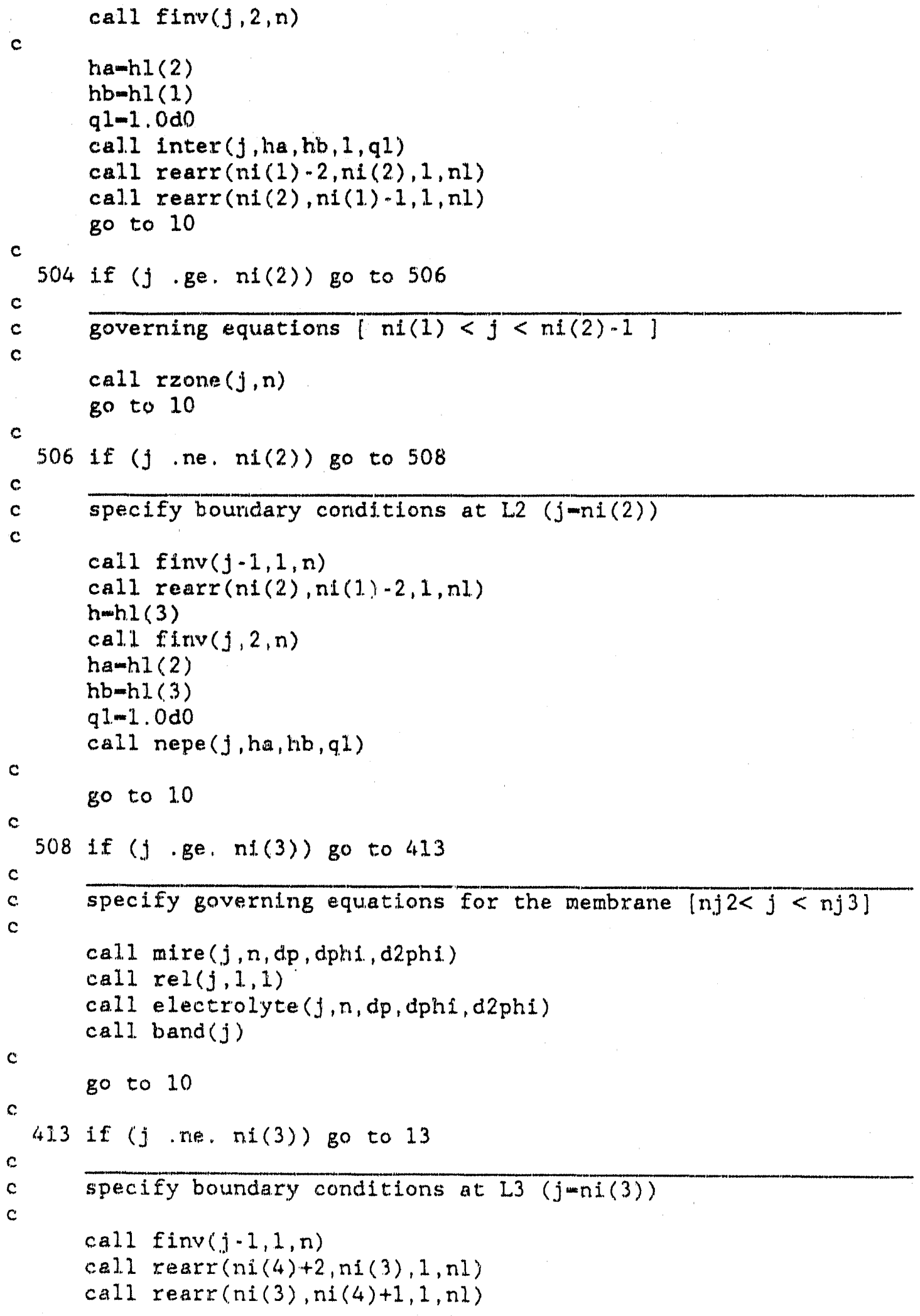


c

$\mathrm{h}=\mathrm{hl}(4)$

call $\operatorname{finv}(j, 2, n)$

hamhl(3)

$\mathrm{hb}=\mathrm{hl}$ (4)

q1 $=1.0 \mathrm{do}$

call nepe ( $j, h a, h b, q l)$

$c$

nk $=2$

phi-phi2

go to 10

13 if (j.ge. ni(4)) go to 16

c

$c$

c

specify governing equations $[n j 3<j<n j 4] / 700 /$

call rzone $(j, n)$

go to 10

16 if ( $j$, ne. $n i(4))$ go to 108

c

c

specify boundary conditions at $L_{4}(j=n(4))$

call rearr $(\operatorname{ni}(3), \operatorname{ni}(4)+2,1, n 1)$

call finv $(j-1,1, n)$

call rearr $(\mathrm{ni}(4), \mathrm{ni}(3)-1, \mathrm{nl}+1, \mathrm{~nm})$

$h=h l(5)$

call $\operatorname{finv}(j, 2, n)$

$c$

hamh I (4)

hb $=h 1$ (5)

q1--1.0d0

call inter $(j, h a, h b,-1, q l)$

c

go to 1.0

108 if $(j$.eq. njj) go to 110

c. spenify governing equations $[n j 4<j<n j 5]$

call $\operatorname{ginv}(j,-1, q 1)$

call $\operatorname{rel}(j, 1,1)$

call gas $(j)$

call band $(j)$

go to 10

$c$

c specify boundary conditions at right interface $(j-m i(5))$

C

110 continue

c 
c

call rearr $(\mathrm{ni}(4), \mathrm{ni}(4)-1, \mathrm{n} 1+1, \mathrm{~nm})$

hd-hl (5)

q1--1.0do

fd-fa

call zbal $(j,-1, q 1, f d$, hd, 2$)$

c

c

c

c

begin check for ss convergence

do $25 \mathrm{i}=1, \mathrm{~nm}$

$\operatorname{xp}(i)=-(4.0 \mathrm{~d} 0 * \mathrm{c}(i, \mathrm{ni}(2)-1)-3.0 \mathrm{~d} 0 * \mathrm{c}(i, \mathrm{ni}(2))-\mathrm{c}(i, \mathrm{ni}(2)-2)) / 2.0 \mathrm{~d} 0 / \mathrm{h}$

do $25 j=1, n j$

if $(c(i, j) .1 t . x x(i, j) / 1.0 d 02) c(i, j)-x x(i, j) / 1.0 d 02$

$x x(n, j)-c(n, j)$

$25 \quad x \times(i, j)-c(i, j)$

c

if ( $j$ count $. g t . I i m$ ) then

c. print 99

call nucamb $(n)$

stop

else

do $55 \quad i 1=2, \mathrm{~nm}$

dxpmadabs ( $x p(i i)-x p 0(i i))$

dxx-dabs ( $x x(i i, n i(2))-x \times 0(i i, n i(2)))$

if $(\mathrm{dxx} \cdot g t \cdot 1,0 \mathrm{~d}-09 * \operatorname{dabs}(\mathrm{xx}(\mathrm{i}, \mathrm{n}, \mathrm{ni}(2)))$ ) go to 8

if ( dxp .gt. 1.0d-06*dabs ( $x p$ (ii) ) . and.

1 $z(i i)$. ne. $0.0 \mathrm{~d} 0$ ) go to 8

c

55 continue

c

end if

c

c print $*, '$,

c. print *, jcount, ' iterations were reqd for ss convergence'

c print $*, '$,

$c$

return

end

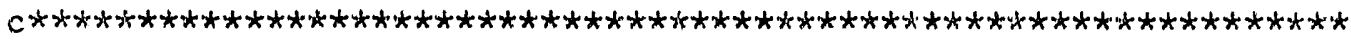

subroutine band $(j)$

implicit real $* 8(a-h, 0-z)$

cominon/n/ $n x, n n, n j, n j j, n l, n g, n i(6), n k$

common/mat. $b, d$

coinmon/band/ $a, c, g, x, y$

dimension $b(26,26), d(26,53)$

dimension $a(26,26), c(26,321), g(26), x(26,26), y(26,26)$

dimension e $(26,27,321)$

101 format ( $15 \mathrm{~h}$ deternmo at $j=, 14$ ) 


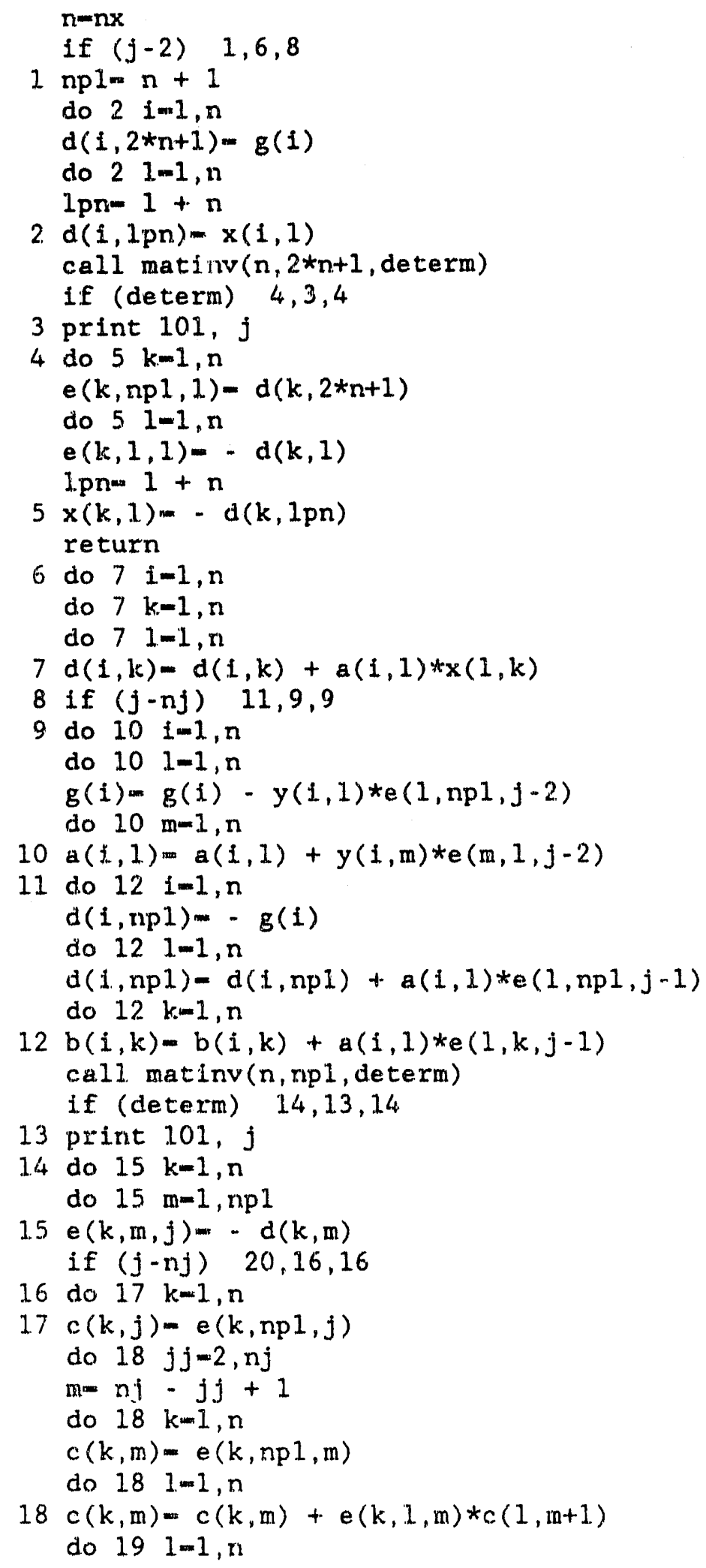




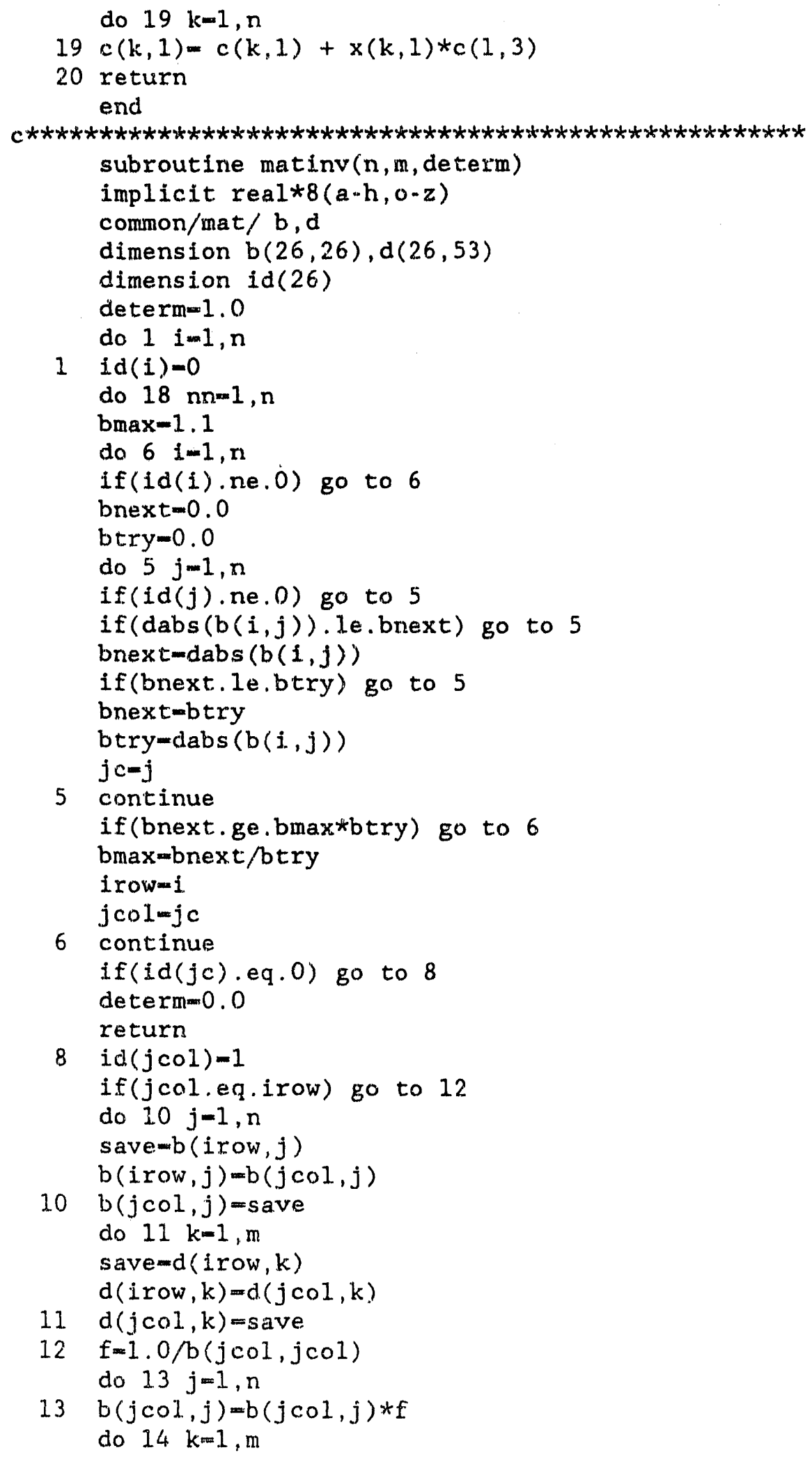


$14 d(j \circ 01, k)=d(j c 01, k) * f$

do $18 \quad i=1, n$

if(i.eq.jcol) go to 18

$f=b(i, j \operatorname{col})$

do $16 j-1, n$

$16 b(i, j)-b(i, j)-f * b(j c o l, j)$

do $17 \mathrm{~km}, \mathrm{~m}$

$17 d(i, k)-d(i, k)-f * d(j c o l, k)$

18 continue

return

end

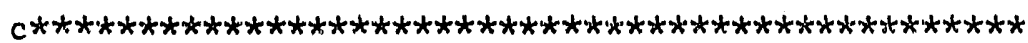

subroutine $z \operatorname{bal}(j, j 1, q 1, f d, h d, i 1)$

implicit real*8 $(a-h, 0-z)$

common/n/ nx, nu, nj, njj, nl, ng, ni (6), nk

common/calc/ h, hl(6)

common/pdata/ $z(12)$, wt $(12)$, ae $(6,2), \mathrm{be}(6,2), \mathrm{ce}(6,2), \mathrm{ee}(6,2)$

$1, \operatorname{ww}(6,2), \operatorname{sd}(6,2), \operatorname{ed}(6,2)$

comnon/prope/dif $(12,12,321)$

common/var/ cc(321), $\mathrm{xx}(13,321), \mathrm{xi}(13,2), \mathrm{flux}(12,321)$

common/comp/s1(13),s2(13),s3(26,26),s4(13),s5(13), binv(13,13)

common/mat/b, d

common/band/a, $c, g, x, y$

dimension $b(26,26), d(26,53)$

dimension $a(26,26), c(26,321), g(26), x(26,26), y(26,26)$

c

call ginv $(j, j 1, q 1)$

call $\operatorname{rel}(j, 1,1)$

do $722 \quad i=n 1+1, n m$

$s 1(i)=0.0 \mathrm{~d} 0$

$s 2(i)=0.0 \mathrm{~d} 0$

do $722 \mathrm{~km}=\mathrm{n} 1+1, \mathrm{~nm}$

$s i(i)-s i(i)+b i n v(k, i)$

if(k.eq.i) go to 722

722 continue

$s 2(i)=s 2(i)+f l u x(k, j) / \operatorname{dif}(i, k, j)$

c

do $715 i=n 1+2, \mathrm{~nm}$

do $727 \mathrm{knn} \mathrm{nl}+2, \mathrm{~nm}$

$a 2=q 1 *(b i n v(i, k)-c(i, j) * s 1(k))$

$b(i, k)=b(i, k)-a 2 * s 2(k)$

$g(i)=g(i)-c(k, j) * a 2 * s 2(k)$

do $7121-n 1+1, n m$

if (1.eq.k) go to 712

$b(i, 1)-b(i, 1)+a 2 * f l u x(k, j) / \operatorname{dif}(k, 1, j)$

$g(i)=g(i)+a 2 * c(1, j) * f l u x(k, j) / \operatorname{dif}(k, 1, j)$

712 continue

$a l=(c(i, j) * s l(k)-b i n v(i, k)) / h d$

$b(i, k)=b(i, k)+1.5 d 0 * a 1$

if (q1.gt. 0.0) then 


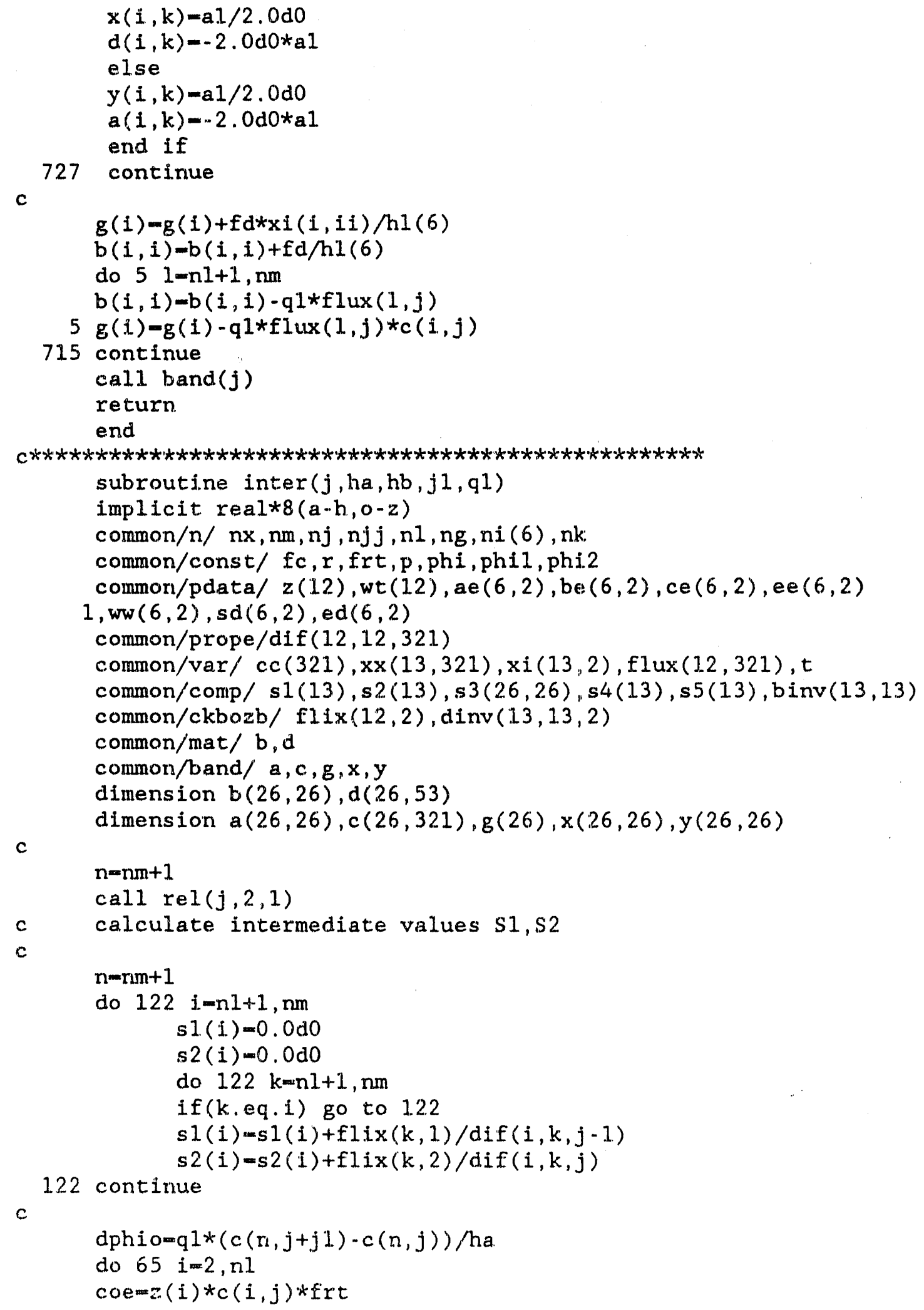




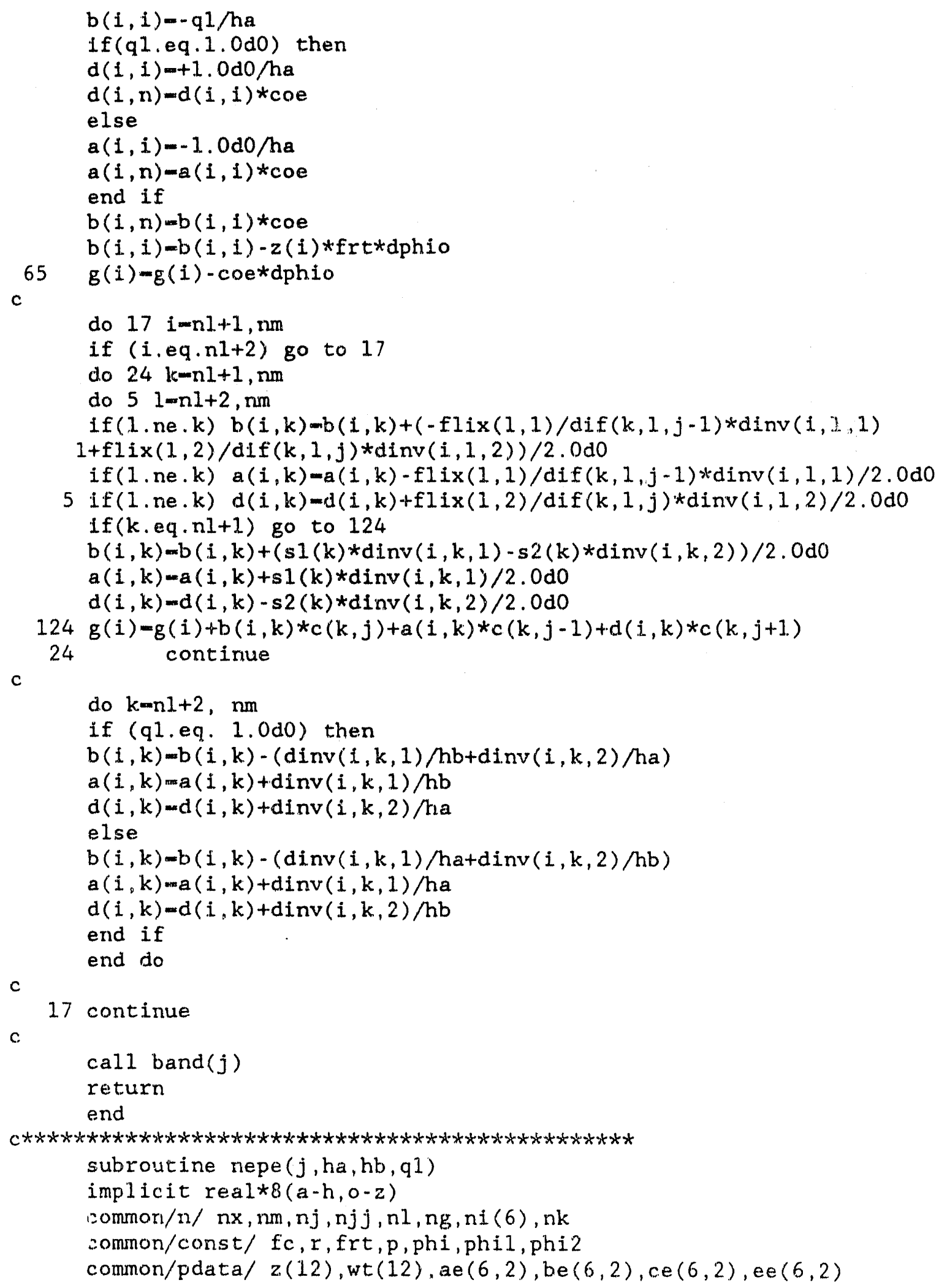




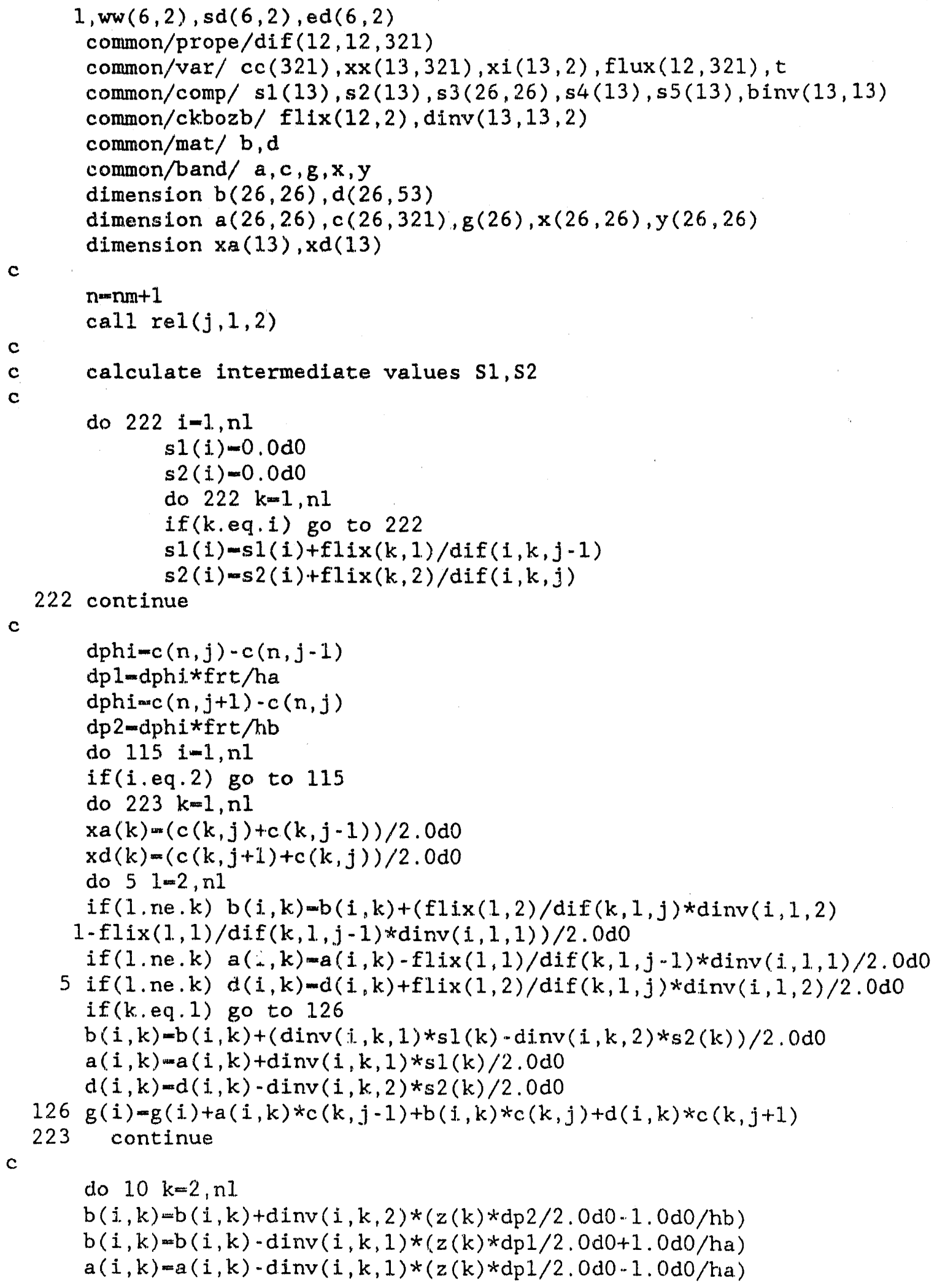




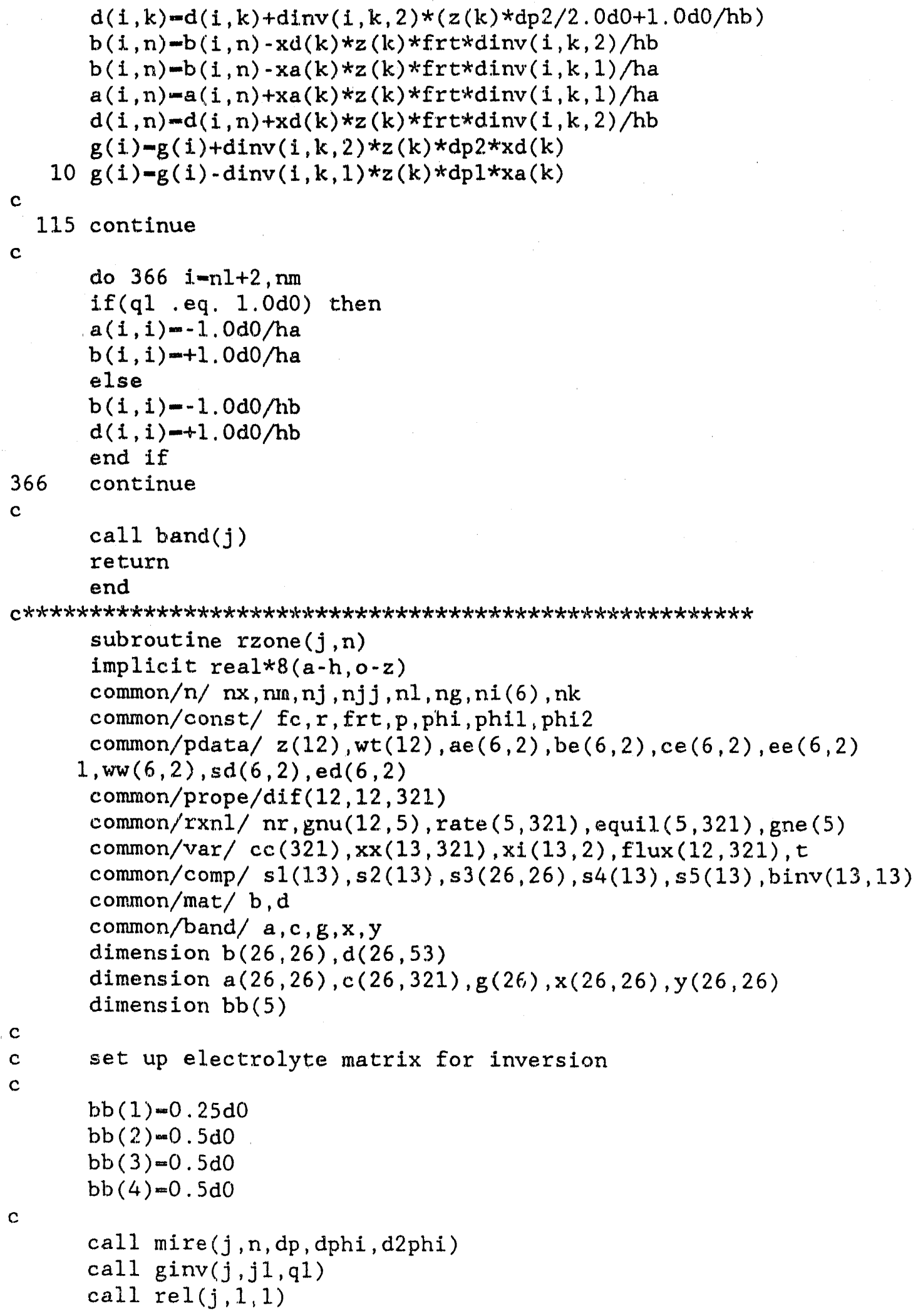




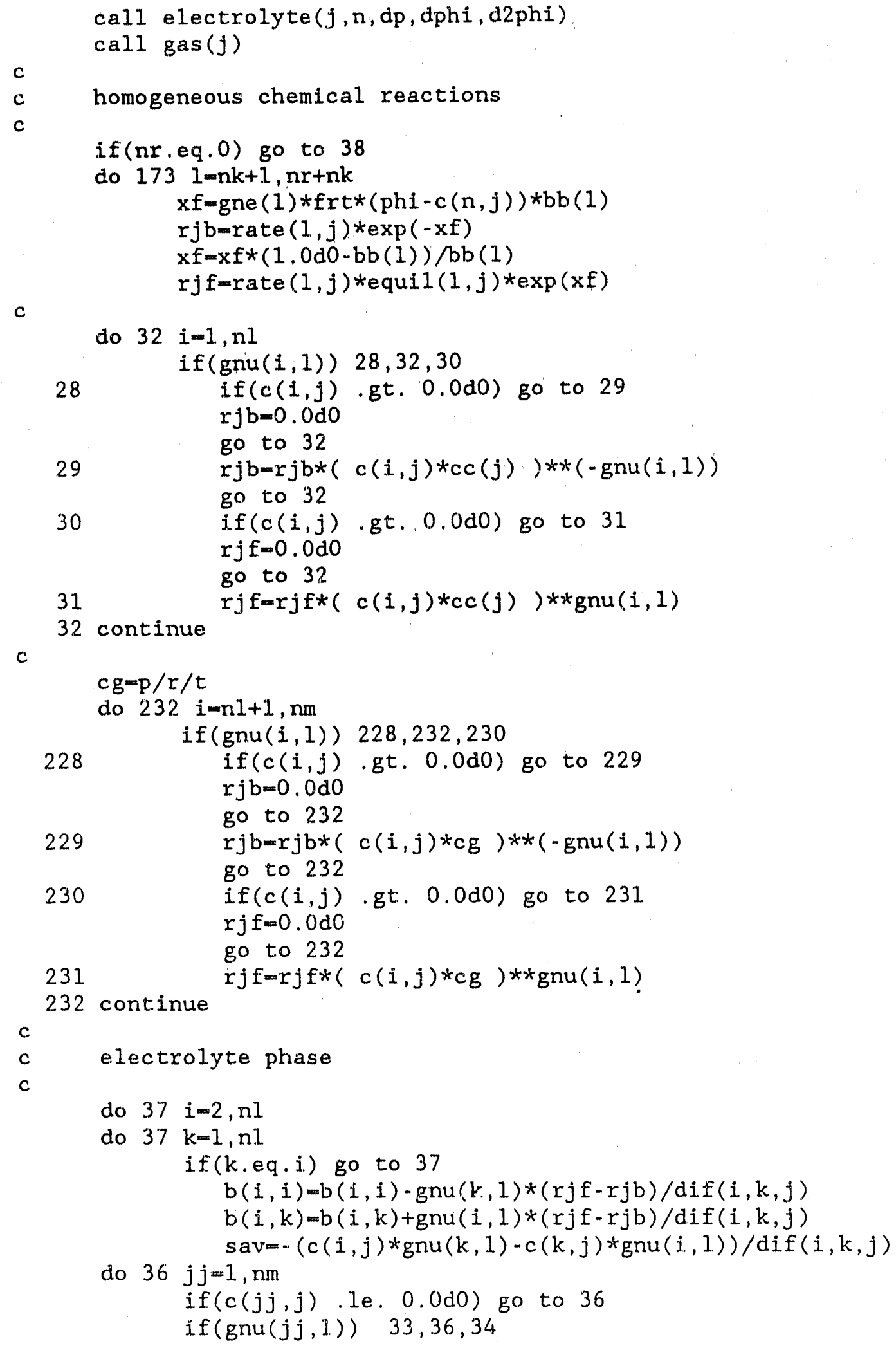




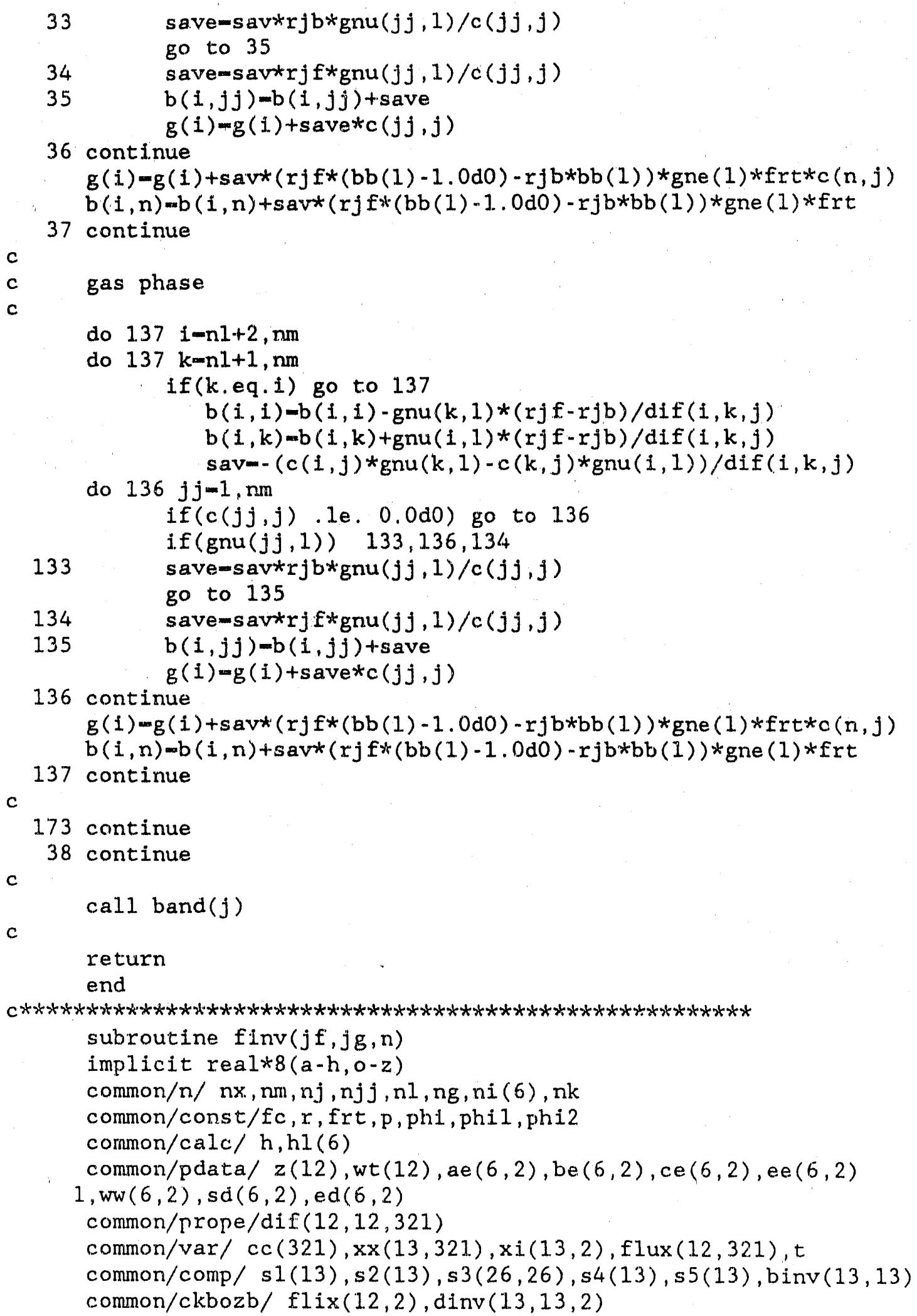




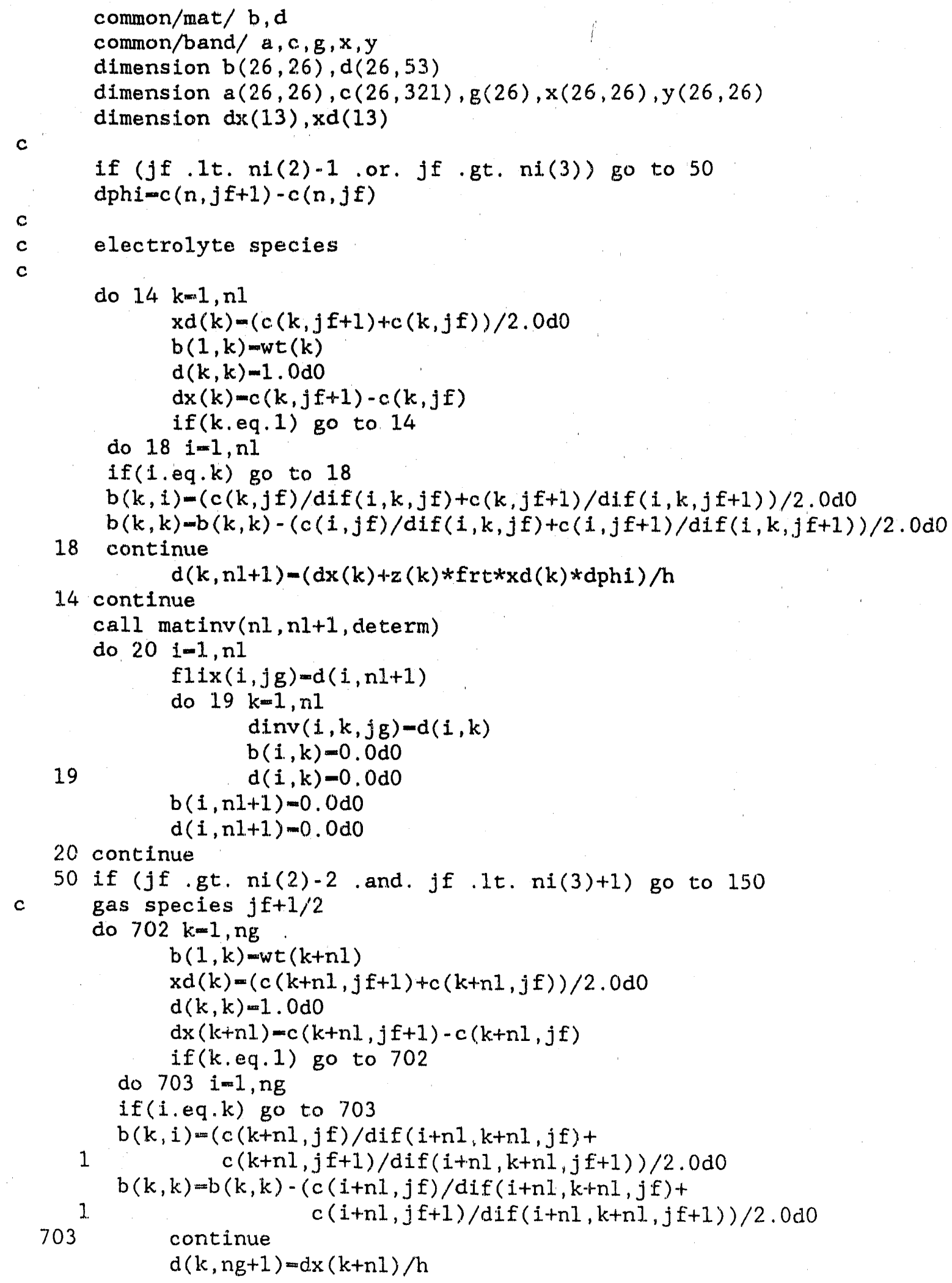




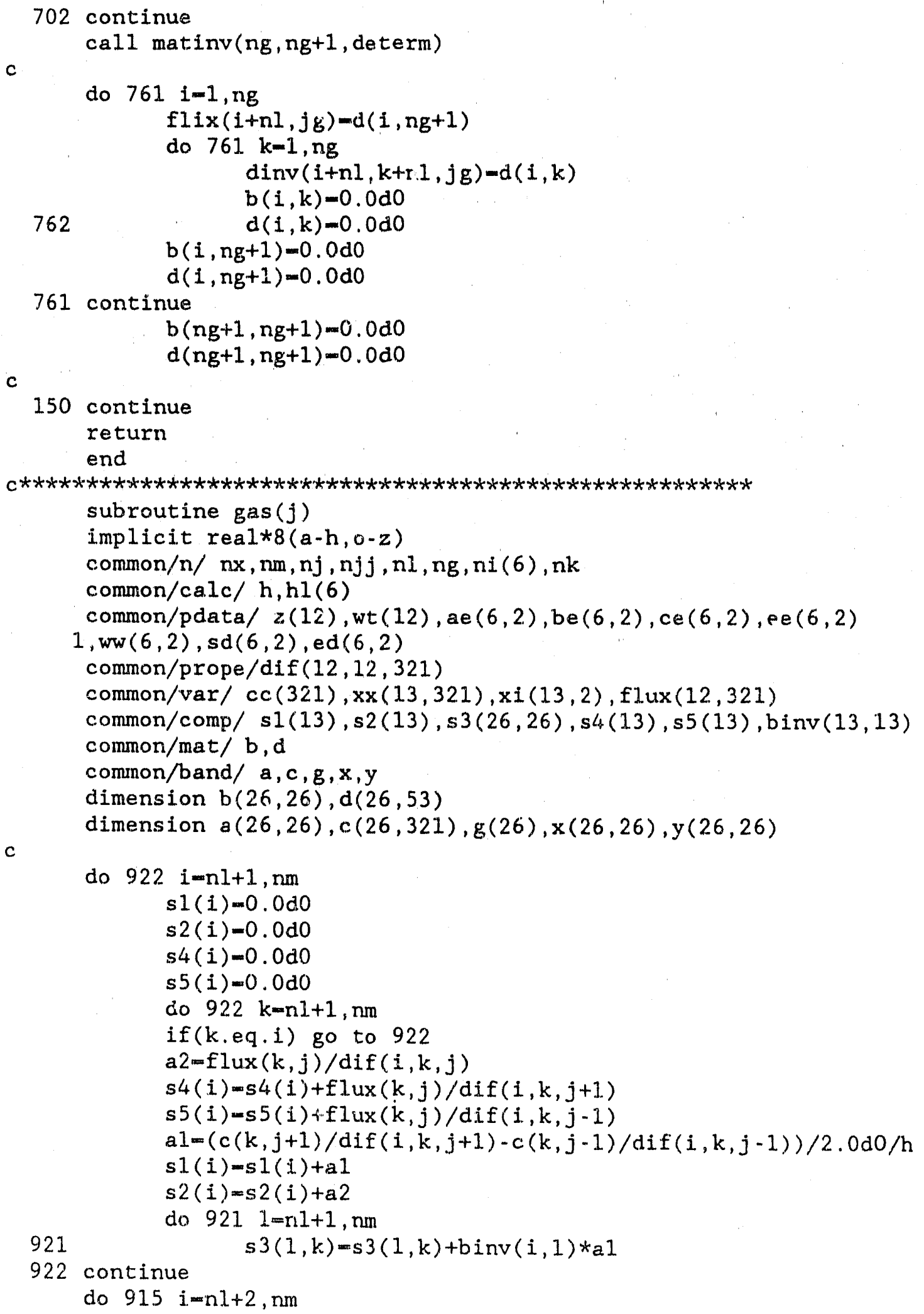


905

927

923

924

915 continue

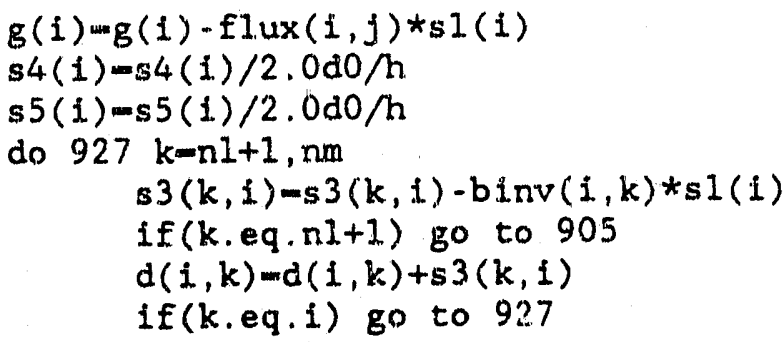


18

1.4 continue

if(k.eq.1) go to 14

do $18 \quad i=1, n]$

if (i.eq.k) go to 18

$b(k, i)-c(k, j) / \operatorname{dif}(i, k, j)$

$b(k, k)-b(k, k)-c(i, j) / \operatorname{dif}(i, k, j)$

continue

$d(k, n l+1)=(d x(k)+z(k) *$ frt $t * c(k, j) * d p h i) / 2.0 d 0 / h$

call 1 matinv( $n l, n 1+1$, determ)

do $20 i-1, n l$

$\operatorname{flux}(i, j)=d(i, n i+1)$

do $19 \mathrm{k}=1, \mathrm{nl}$

binv $(i, k)-d(i, k)$

19

$b(i, k)=0.0 \mathrm{~d} 0$

20 $d(i, k)=0.0 \mathrm{~d} 0$

$b(1, n 1+1)=0.0 d 0$

$\mathrm{d}(i, \mathrm{n} .1+1)=0.0 \mathrm{~d} 0$

return

end

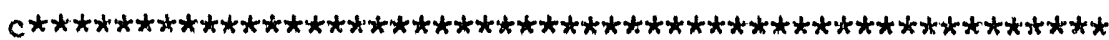

subroutine electrolyte $(j, n, d p, d p h i, d 2 p h i)$

implicit real*8(a-h,0-z)

common/n/ $n x, n m, n j, n j j, n l, n g, n i(6), n k$

common/const/ fc, $r, f r t, p, p h i, p h i 1, p h i 2$

common/calc/h,hI(6)

common/pdata/ $z(12)$, wt ( 12$)$, ae $(6,2)$, be $(6,2), \mathrm{ce}(6,2)$, ee $(6,2)$

$1, \operatorname{ww}(6,2), \operatorname{sd}(6,2), \operatorname{ed}(6,2)$

common/prope/dif $(12,12,321)$

common/var/ cc(321), xx(13,321), xi(13,2), flux $(12,321), t$

common/comp/s1(13),s2(13),s3(26,26), s4(13), s5(13), binv $(13,13)$

common/mat/b,d

common/band/ a, c, $g, x, y$

dimension $b(26,26), d(26,53)$

dimension $a(26,26), c(26,321), g(26), x(26,26), y(26,26)$

dimension $\mathrm{dx}(13)$

do $722 \quad i-1, n 1$

$s 1$ (i) $-0.0 \mathrm{~d} 0$

$s 2(1)=0.0 \mathrm{do}$

$s 4(i)=0.0 \mathrm{dO}$

s5(i) $=0.0 \mathrm{do}$

do $722 k=1, n 1$

if (k.eq.1) go to 722

a2 $-f \operatorname{lux}(k, j) / \operatorname{dif}(i, k, j)$

$s 4(i)-s 4(i)+f \operatorname{lux}(k, j) / \operatorname{dif}(i, k, j+1)$

$s 5(i)-s 5(i)+f \operatorname{lux}(k, j) / \operatorname{dif}(i, k, j-1)$

al- $(c(k, j+1) / \operatorname{dif}(i, k, j+1)-c(k, j-1) / \operatorname{dif}(i, k, j-1)) / 2.0 \mathrm{~d} 0 / \mathrm{h}$

si (i) $=s i(i)+a l$

$s 2(i)-s 2(i)+a 2$ 
722 continue

do $715 \quad 1=2, \mathrm{nl}$

$g(i)-g(i)-f l u x(i, j) * s I(i)$

$s 4(i)=54(i) / 2.0 \mathrm{~d} 0 / \mathrm{h}$

$\mathrm{s} 5(i)-s 5(1) / 2.0 \mathrm{~d} 0 / \mathrm{h}$

do $72 ; k=1, n 1$

$s 3(k, i)-s 3(k, i)-b i n v(i, k) * s 1(i)$

if (k.eq.1) go to 705

$d(i, k)-d(i, k)+s 3(k, i)$

$d(i, n)-d(i, n)+z(k) * c(k, j) * f r t * s 3(k, i) / 2.0 d 0 / h$

705 $a(i, n)-a(1, n)-z(k) * c(k, j) * f r t * s 3(k, i) / 2.0 d 0 / h$

727

continue if (k.eq.i) go to 727

do $724 \mathrm{k}=1, \mathrm{n} 1$

$d(i, k)-d(i, k) / 2.0 d 0 / h$

$a(i, k)-d(i, k)$

723

do $7231-2, n l$

if $(1$.ne.k) $b(i, k)-b(i, k)+f l u x(1, j) / \operatorname{dif}(k, 1, j) * s 3(1, i)$

if $(k, n e .1) b(i, k)-b(i, k)-s 2(k) * s 3(k, i)+s 3(k, i) * z(k) * d p$ if (k.eq.i) go to 724

$g(i)-g(i)+b(i, k) * c(k, j)$

$d(i, k)-d(i, k)-f \operatorname{lux}(i, j) / d i f(i, k, j+1) / 2.0 d 0 / h$

724 continue $a(1, k)-a(i, k)+f \operatorname{lux}(i, j) / \operatorname{dif}(i, k, j-1) / 2.0 \mathrm{~d} 0 / \mathrm{h}$

$d(i, i)=d(i, i)+s 4(i)-1.0 \mathrm{~d} 0 / \mathrm{h} / \mathrm{h}-z(i) * 2 \mathrm{p} / 2.0 \mathrm{~d} 0 / \mathrm{h}$

$a(i, i)=a(i, i)-s 5(i)-1.0 \mathrm{do} / \mathrm{h} / \mathrm{h}+\mathrm{z}(\mathrm{i}) * \mathrm{dp} / 2.0 \mathrm{~d} 0 / \mathrm{h}$

1

$g(i)-g(i)+b(i, i) * c(i, j)+s 4(i) * c(i, j+1)-s 5(i) * c(i, j-1)$ $-z(i) * f r t *(c(i, j) * d 2 p h i+0.25 * \mathrm{dx}(i) * d p h i) / \mathrm{h} / \mathrm{h}$

$a(i, n)-a(i, n)+z(i) * f r c *(d x(i) / 4.0 d 0-c(i, j)) / h / h$

$b(i, n)-z(i) * f r t * 2.0 d 0 * c(i, j) / h / h$

$d(i, n)=d(i, n)-z(i) * f r t *(d x(i) / 4.0 d 0+c(i, j)) / h / h$

715 continue

$b(i, i)=b(i, i)+2.0 d 0 / h / h-d 2 p h i * z(i) * f r t / h / h$

return

end

$c * * * * * * * * * * * * * * * * * * * * * * * * * * * * * * * * * * * * * * * * * * * * * * * * * * *$

subroutine rearr $(j i, j j, k 1, k 2)$

implicit $x e a] * B(a-h, o-z)$

common/pdata/ $z(12), w t(12)$, ae $(6,2)$, be $(6,2)$, ce $(6,2)$, ee $(6,2)$

$1, w w(6,2), s d(6,2), e d(6,2)$

common/prope/dif $(12,12,321)$

do $5 \mathrm{~km}-\mathrm{k} 1, \mathrm{k} 2$

do $51 \mathrm{k} k 1, \mathrm{k} 2$

if $(k, e q .1)$ go t.o 5

$\operatorname{dif}(k, 1, i j)=\operatorname{dif}(k, 1, j j)$

5 continue

return

end

$c * * * * * * * * * * * * * * * * * * * * * * * * * * * * * * * * * * * * * * * * * * * * * * * * * * * * * * *$

subroutine $r e l(j, k k, I I)$ 


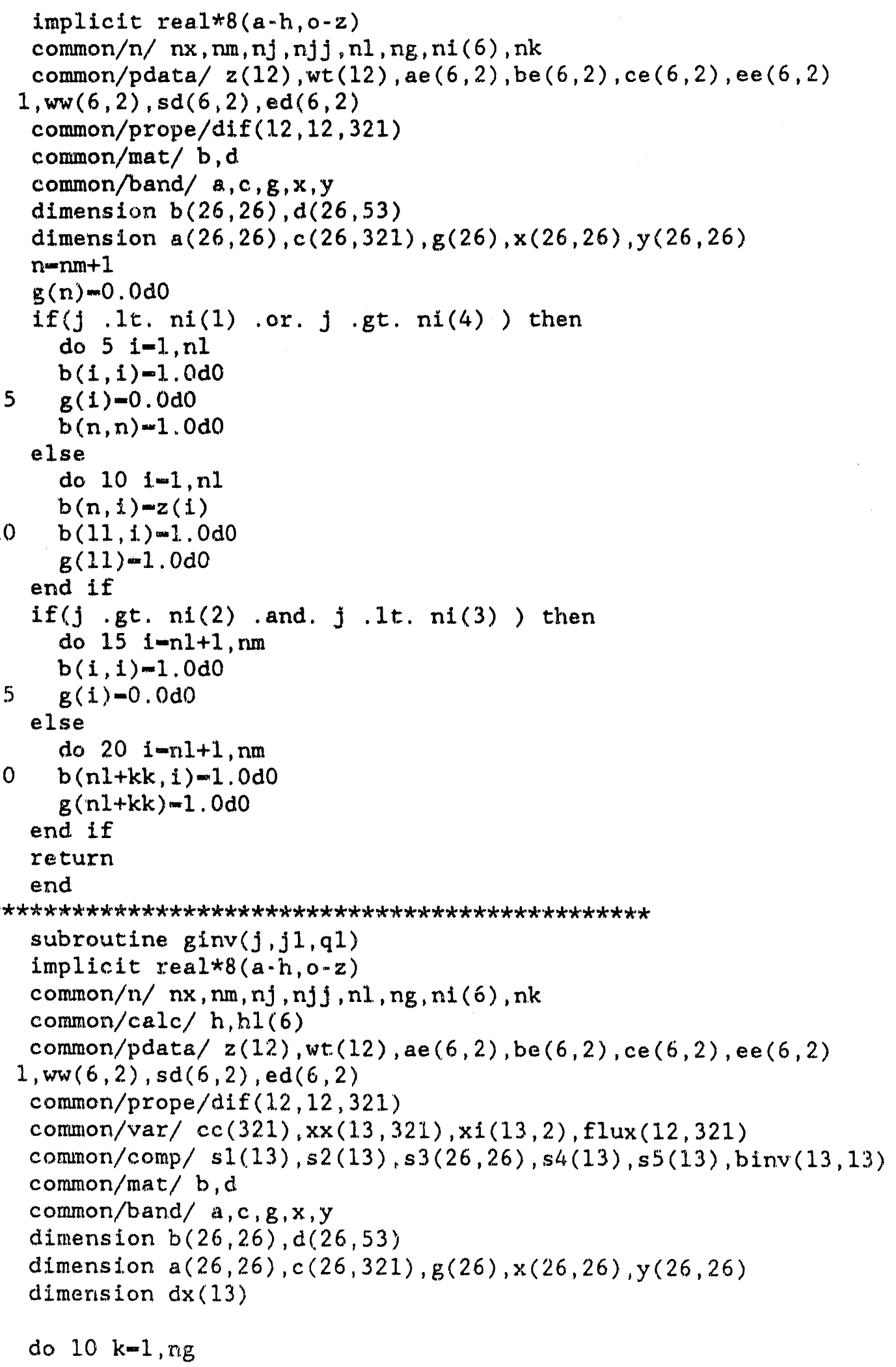




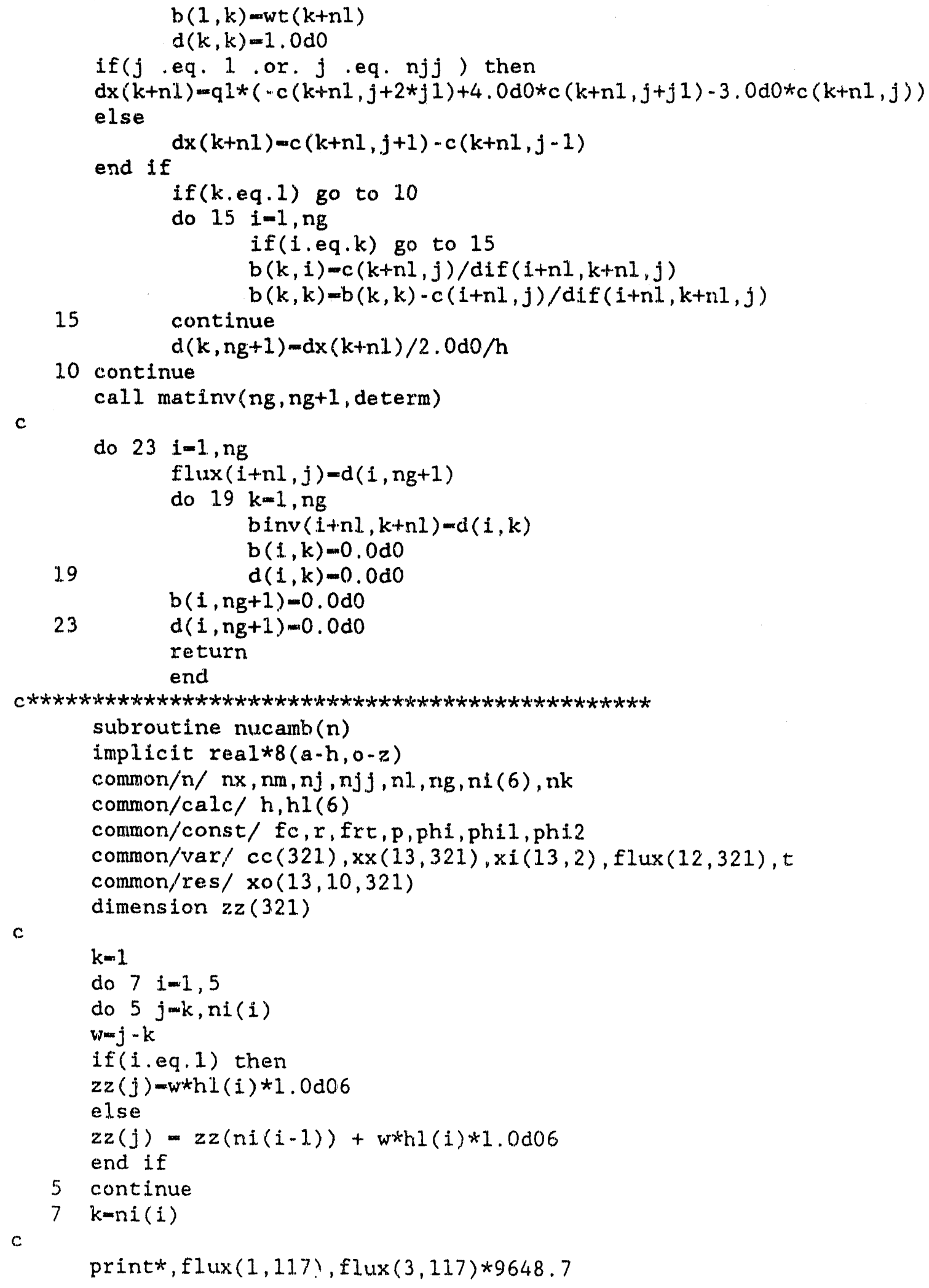




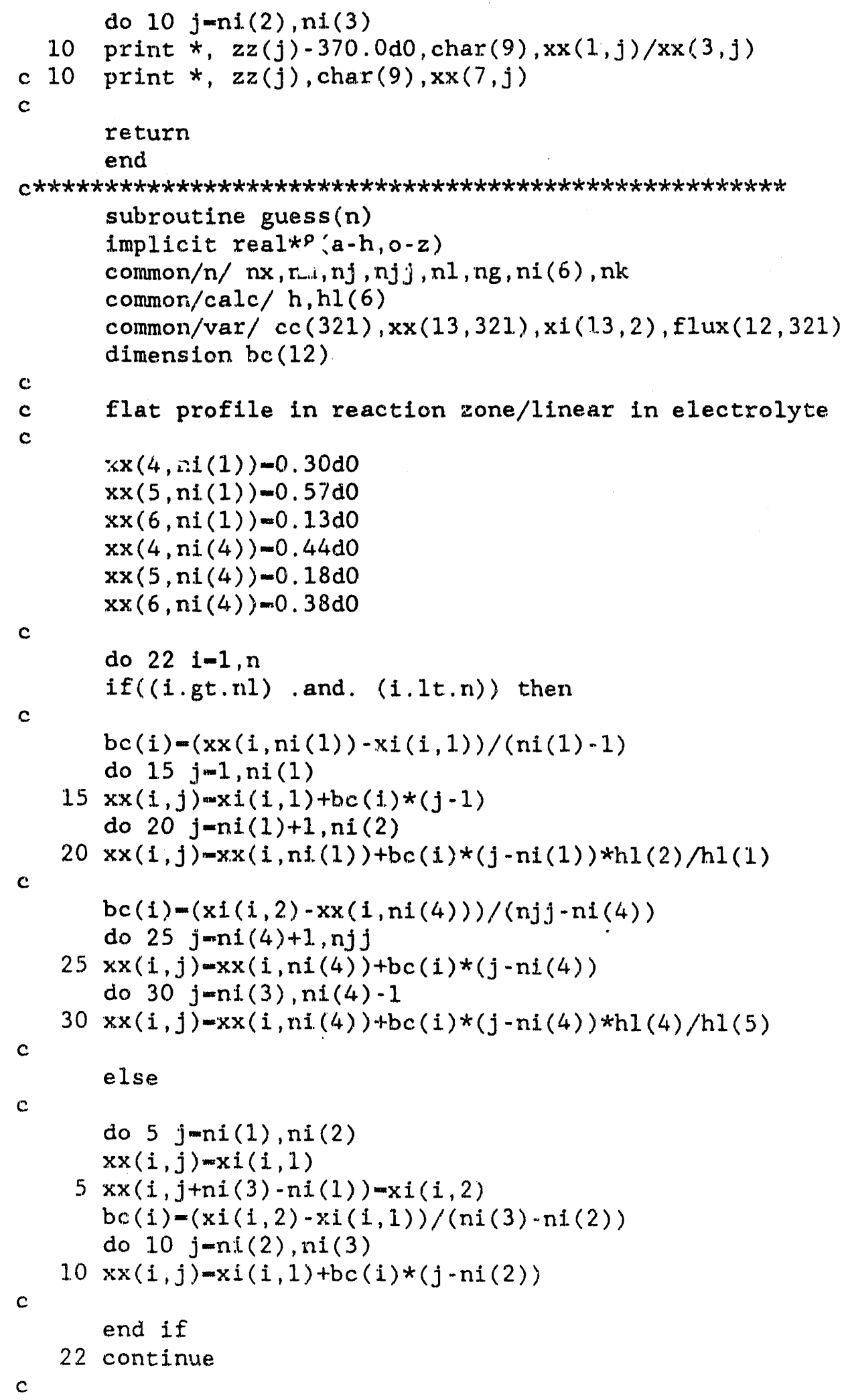




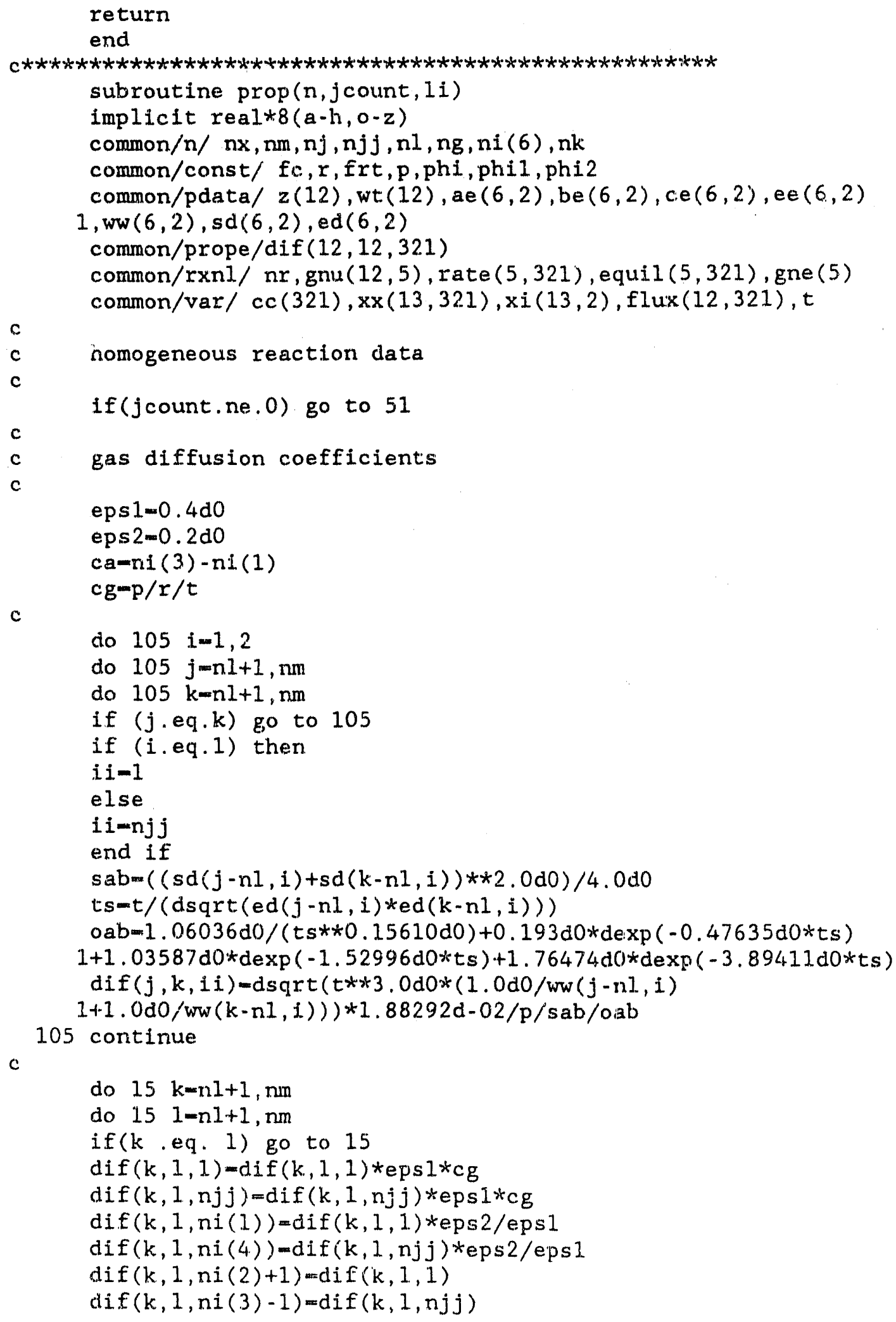




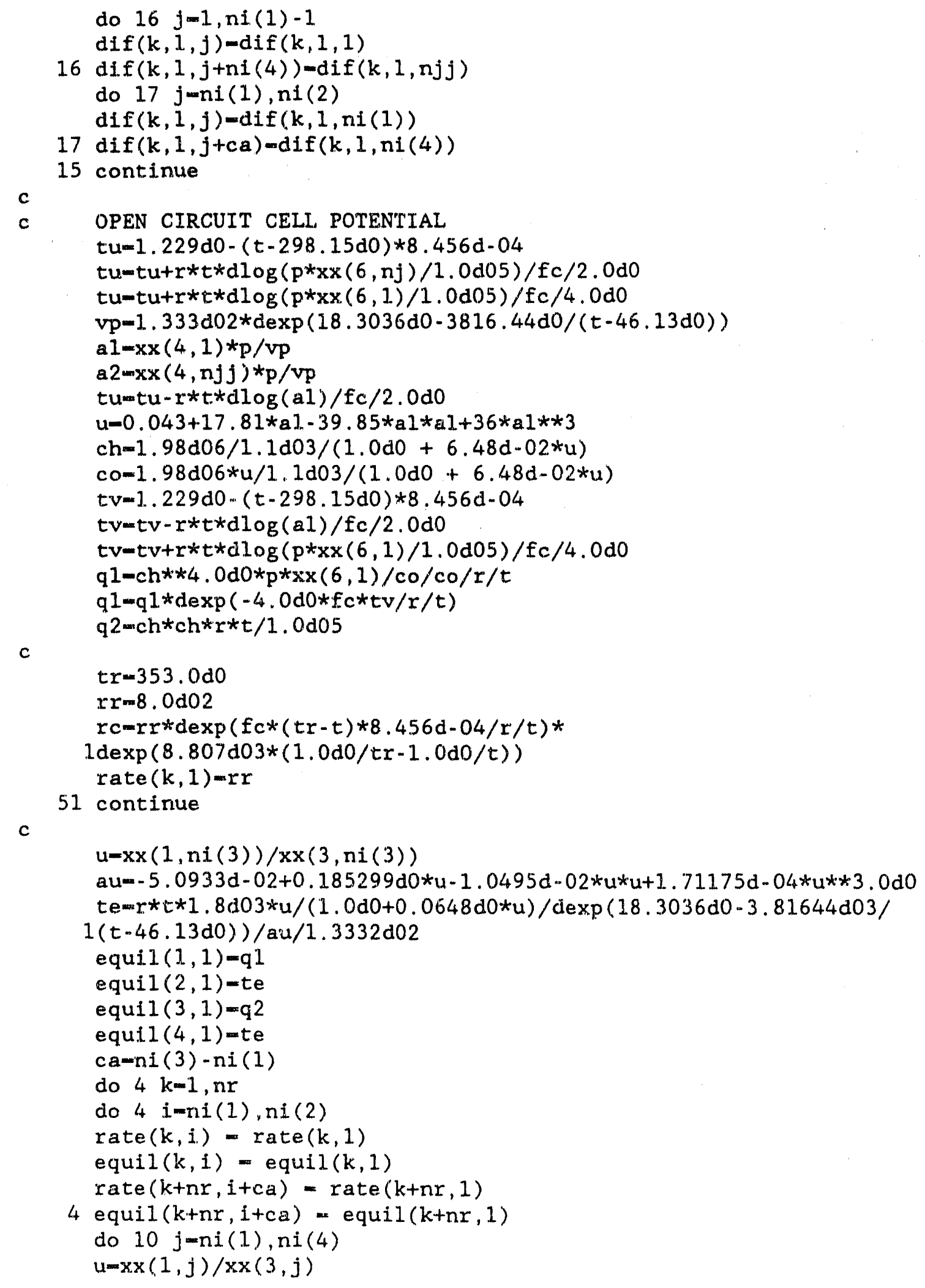


if $(j$ count . eq. 0 and. $1 i$.eq. 1$) u=9.0 \mathrm{~d} 0$

$\mathrm{co}=1.98 \mathrm{~d} 03 * \mathrm{u} / 1.1 \mathrm{~d} 03 /(1.0 \mathrm{~d} 0+6.48 \mathrm{~d}-02 * \mathrm{u})$

$\mathrm{ch}=1.98 \mathrm{~d} 03 / 1.1 \mathrm{~d} 03 /(1.0 \mathrm{~d} 0+6.48 \mathrm{~d}-02 * \mathrm{u})$

$\mathrm{cm}=1.98 \mathrm{~d} 03 / 7.7 \mathrm{~d} 01 /(1.0 \mathrm{~d} 0+6.48 \mathrm{~d}-02 * \mathrm{u})$

$c c(j)=(2.751 \mathrm{~d} 04+1.8 \mathrm{~d} 03 * u) /(1.0 \mathrm{~d} 0+6.48 \mathrm{~d}-02 * u)$

ek $=(3.375 d-01 * u-0.2141 d 0) * d \exp (-1.268 \mathrm{~d} 03 / t)$

$\mathrm{dp}=((0.35 \mathrm{~d} 0 * \mathrm{u}) * *(-4.0 \mathrm{~d} 0)+0.2142 \mathrm{~d} 0) * *(-0.25 \mathrm{~d} 0)$

$\mathrm{r} 0-(1.98 \mathrm{~d} 03+32.4 \mathrm{~d} 0 * u) /(1.0 \mathrm{~d} 0+6.48 \mathrm{~d}-02 * u)$

af $=0.04 \mathrm{~d} 0 * \mathrm{r} * t *(((\mathrm{u}+61.11 \mathrm{~d} 0) * * 2) / \mathrm{u} / \mathrm{u}+1.0 \mathrm{~d} 0)$

$\mathrm{df}=\mathrm{r} 0 *(3.5 \mathrm{~d}-06 * \exp (-2.436 \mathrm{~d} 03 / \mathrm{t})) / \mathrm{af}$

$\mathrm{df}=\mathrm{df} * \mathrm{u} *(\mathrm{u}+61.11 \mathrm{~d} 0) / 1.10 \mathrm{~d} 0 / 14.0 \mathrm{~d} 0$

c

calculate Lij's

$a=e k * d p / f c / f c / c h / c o / 1.0 d 04$

c

$b=d f / c o / c o / 1.0 d 06+e k * d p * d p / c o / c o / f c / f c / 1.0 d 04$

$c$

$c=e k / f c / f c / c h / c h / 1.0 d 04$

c

c

c

calculate Kij's

$x 1-a_{1}^{\prime}(-a * a+b * c)$

y1 $=(c-a) /(-a * a+b * c)$

$z 1=(b-a) /(-a * a+b * c)$

c

c calculate Dij's

eps $=1.0 \mathrm{~d} 0$

if $(j .1 t \cdot n i(2)$.or. $j \cdot g t \cdot n i(3))$ eps $=0.2 d 0$

$\operatorname{dif}(1,3, j)=1.0 \mathrm{~d} 06 *$ eps*r*t*co*ch $/ \mathrm{x} 1$

$\operatorname{dif}(2,3, j)=1.0 \mathrm{~d} 06 *$ eps $* \mathrm{r} * \mathrm{t} * \mathrm{ch} * \mathrm{~cm} / \mathrm{z} 1$

$\operatorname{dif}(1,2, j)=1.0$ d06*eps $* 1 * t * \operatorname{co} * \mathrm{~cm} / \mathrm{y} 1$

$\operatorname{dif}(2,1, j)-\operatorname{dif}(1,2, j)$

$\operatorname{dif}(3,1, j)=\operatorname{dif}(1,3, j)$

$10 \operatorname{dif}(3,2, j)=\operatorname{dif}(2,3, j)$

do $34 \mathrm{k}=1, \mathrm{nl}$

do $341=1, n 1$.

if $(k$.eq. 1) go to 34

$\operatorname{dif}(k, 1, \operatorname{ni}(1)-1)=\operatorname{dif}(k, 1, n i(2)-1)$

$\operatorname{dif}(k, 1, \operatorname{ni}(4)+1)=\operatorname{dif}(k, 1, \operatorname{ni}(3)+1)$

34 continue

return

end

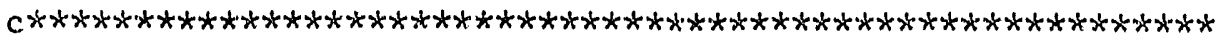

subroutine heat $(t 1, t 2, f a, f b)$

implicit real*8(a-h,o-z)

common/n/ nx, nm, nj, njj, nl, ng, ni(6), nk

common/const/ fc, r, frt, p,phi,phil, phi2

common/calc/ h, hl(6) 
common/pdata/ $z(12), \operatorname{wt}(12)$, ae $(6,2), \operatorname{be}(6,2), \operatorname{ce}(6,2)$, ee $(6,2)$

$1, \operatorname{ww}(6,2), \operatorname{sd}(6,2), \operatorname{ed}(6,2)$

c common/var/ $\operatorname{cc}(321), x \times(13,321), x i(13,2)$, flux $(12,321), t$
$t r-298.15 \mathrm{~d} 0$
$\operatorname{tam} 298.15 \mathrm{~d} 0$
hc $-3.6 \mathrm{~d} 01$

c

a $1=0.0 \mathrm{~d} 0$
a2 $=0.0 \mathrm{~d} 0$
b1 $=0.0 \mathrm{~d} 0$
b2 $=0.0 \mathrm{~d} 0$
c1 $1=0.0 \mathrm{~d} 0$
c $2=0.0 \mathrm{~d} 0$
df $=0.0 \mathrm{~d} 0$

do $5 \mathrm{i}=1, \mathrm{~nm}-\mathrm{nl}$

df-df - (flux $(i+n l, 1) * e e(i, 1)-f l u x(i+n l, n j j) * e e(i, 2)) * h I(6)$

$a 1-a 1+f b * x i(i+n 1,1) * a e(i, 1)+f a * x i(i+n 1,2) * a e(i, 2)$

$a 2-a 2+(f b * x i(i+n 1,1)-f 1 u x(i+n 1,1) * h 1(6)) * a e(i, 1)+$

$1(f a * x i(i+n 1,2)+f l u x(i+n 1,2) * h 1(6)) * a e(i, 2)$

b1-b1+fb*xi $(i+n 1,1) * b e(i, 1)+f a * x i(i+n l, 2) * b e(i, 2)$

b2-b2+(fb*xi $(i+n l, 1)-f l u x(i+n l, 1) * h l(6)) * b e(i, 1)+$

$1($ fa*xi $(i+n l, 2)+f l u x(i+n l, 2) * h l(6)) * b e(1,2)$

$c 1-c 1+f b * x i(i+n 1,1) * c e(i, 1)+f a * x i(i+n 1,2) * c e(i, 2)$

$5 \mathrm{c} 2-\mathrm{c} 2+(\mathrm{fb} * \mathrm{x} i(i+n 1,1)-f 1 u x(i+n l, 1) * h l(6)) * c e(i, 1)+$

c

$1(f a * x i(i+n l, 2)+f l u x(i+n l, 2) * h l(6)) * c e(i, 2)$

$c p 1=a 1 *(t 1-t r)+b 1 *(t 1 * t 1-t r * t r) / 2.0 d 0-c 1 *(t r-t 1) / t r / t 1$ $c p 2=a 2 * t r+b 2 * t r * t r / 2.0 \mathrm{~d} 0-c 2 / t r$

$w=f l u x(3,117) * h 1(6) * f c *(p h i 2-p h i 1)$

$q 1=-h_{i} c * h l(6) *(t-t a)$

$r k=c p 1+c p 2-d f+q 1-w$

$\mathrm{rp}=-2.0 \mathrm{~d} 0 * \mathrm{rk} / \mathrm{b} 2-4.0 \mathrm{~d} 0 * \mathrm{a} 2 * \mathrm{a} 2 / \mathrm{b} 2 / \mathrm{b} 2 / 3.0 \mathrm{~d} 0$

$\mathrm{rq}=-2.0 \mathrm{~d} 0 * \mathrm{c} 2 / \mathrm{b} 2+4.0 \mathrm{~d} 0 * \mathrm{a} 2 * \mathrm{rk} / \mathrm{b} 2 / \mathrm{b} 2 / 3.0 \mathrm{~d} 0+1.6 \mathrm{~d} 0 *(\mathrm{a} 2 / \mathrm{b} 2) * * 3.0 \mathrm{~d} 0 / 2.7 \mathrm{~d} 0$

$u=d s q r t(-27.0 d 0 * r q \star r q / r p / r p / r p / 4.0 d 0)$

$v=$ dacos $(u)$

$v=v / 3.0 \mathrm{dO}+2.0 \mathrm{~d} 0 * 3.1415926535 \mathrm{do} / 3.0 \mathrm{do}$

$\mathrm{yr}=-\mathrm{dsqrt}(-4.0 \mathrm{~d} 0 * \mathrm{rp} / 3.0 \mathrm{~d} 0) * \mathrm{dcos}(\mathrm{v})$

$\mathrm{t} 2-\mathrm{yr}-2.0 \mathrm{~d} 0 * \mathrm{a} 2 / \mathrm{b} 2 / 3.0 \mathrm{do}$

return

end 


\subsection{Introduction}

In this chapter, we are concerned with the transient response of a fuel-cell system. In a recent review [1], Srinivasan points to scale-up, start-up time, and peaking capabilities as the primary concerns for developing fuel cells for transportation applications. High current densities can result in the transport of water from the anode-membrane interface at a rate that is greater than that at which it can be restored by back diffusion or absorption from the gas phase (see the preceding chapter). While continued operation at high current densities can dehydrate the polymer electrolyte and degrade the performance of the cell, short periods of operation at high power may be possible.

Examination of transport in the separator of a solid-polymerelectrolyte fuel cell following a step change in current or voltage should help to elucidate the behavior under these conditions. Here we limit our study to transport in the membrane under isothermal conditions. We also include the response to a small sinusoidal perturbation in current or voltage (see appendix $D$ for an analysis of the impedance). Extension to the porous gas-diffusion electrode and the fuel-cell system is straightforward (see reference [2]). 


\subsection{Transient response}

We consider a membrane of thickness $l$, with an electrode at each end. At the anode-membrane interface, $y=0$, the flux of water is zero and the current is specified. At the cathode-membrane interface thermodynamic equilibrium is assumed. Therefore, at $y=\ell$, the concentration and potential are specified.

The transport equations for the solid polymer electrolyte were developed in chapter two. With the potential defined by $F \Phi-\mu_{+}$,

$$
1--\kappa \nabla \Phi-\frac{\kappa \xi}{F} \nabla \mu_{0}
$$

and

$$
\mathrm{N}_{0}--\frac{\kappa \xi}{F} \nabla \Phi-\left(\alpha+\frac{\kappa \xi^{2}}{F^{2}}\right) \nabla \mu_{0}
$$

A material balance on water gives

$$
\frac{\partial c_{0}}{\partial t}=-\nabla \cdot \mathbf{N}_{0}
$$

The divergence of the current density is zero; therefore,

$$
\kappa \nabla^{2} \Phi-\frac{\kappa \xi}{F} \nabla^{2} \mu_{0}
$$

Taking the divergence of equation 2 and substituting for $\nabla^{2} \Phi$ from equation 4 yield 


$$
\frac{\partial c_{0}}{\partial t}=\alpha \nabla^{2} \mu_{0}=D \nabla^{2} c_{0}
$$

where $D=\frac{\alpha R T}{\bar{c}_{0}}$. Using separation of variables, a series solution is possible; however, we will not pursue this approach here. See Carslaw and Jaeger (p. 104) [3] for further information. We can, nevertheless, obtain an estimate of the diffusion-time constant for the system. For a $175 \mu \mathrm{m}$ membrane and a diffusion coefficient of $3 \times 10^{-2} \mathrm{~cm}^{2} / \mathrm{s}$,

$$
\tau=\ell^{2} / D \approx 10 \mathrm{~s}
$$

The time-dependent equations derived in chapter three were solved numerically using a time-stepping procedure. This approach allows one to use variable physical properties. The Crank-Nicolson method, an implicit method which can be found in most books on numerical methods [4], was used to evaluate the time derivatives. The computer program transient. for is listed in appendix $\mathrm{E}$.

Figures 1 and 2 show the ratio of water concentration to hydrogen ion concentration under transient conditions. The separator had a thickness of $175 \mu \mathrm{m}$. The transport properties are the same as those described in chapter 5. Figure 1 corresponds to zero initial current followed by a step change to $400 \mathrm{~mA} / \mathrm{cm}^{2}$. Figure 2 is the converse and shows the back diffusion of water following steady-state operation. Steady state is reached in about $20 \mathrm{~s}$. A higher value of the diffusion coefficient results in faster back diffusion and allows 


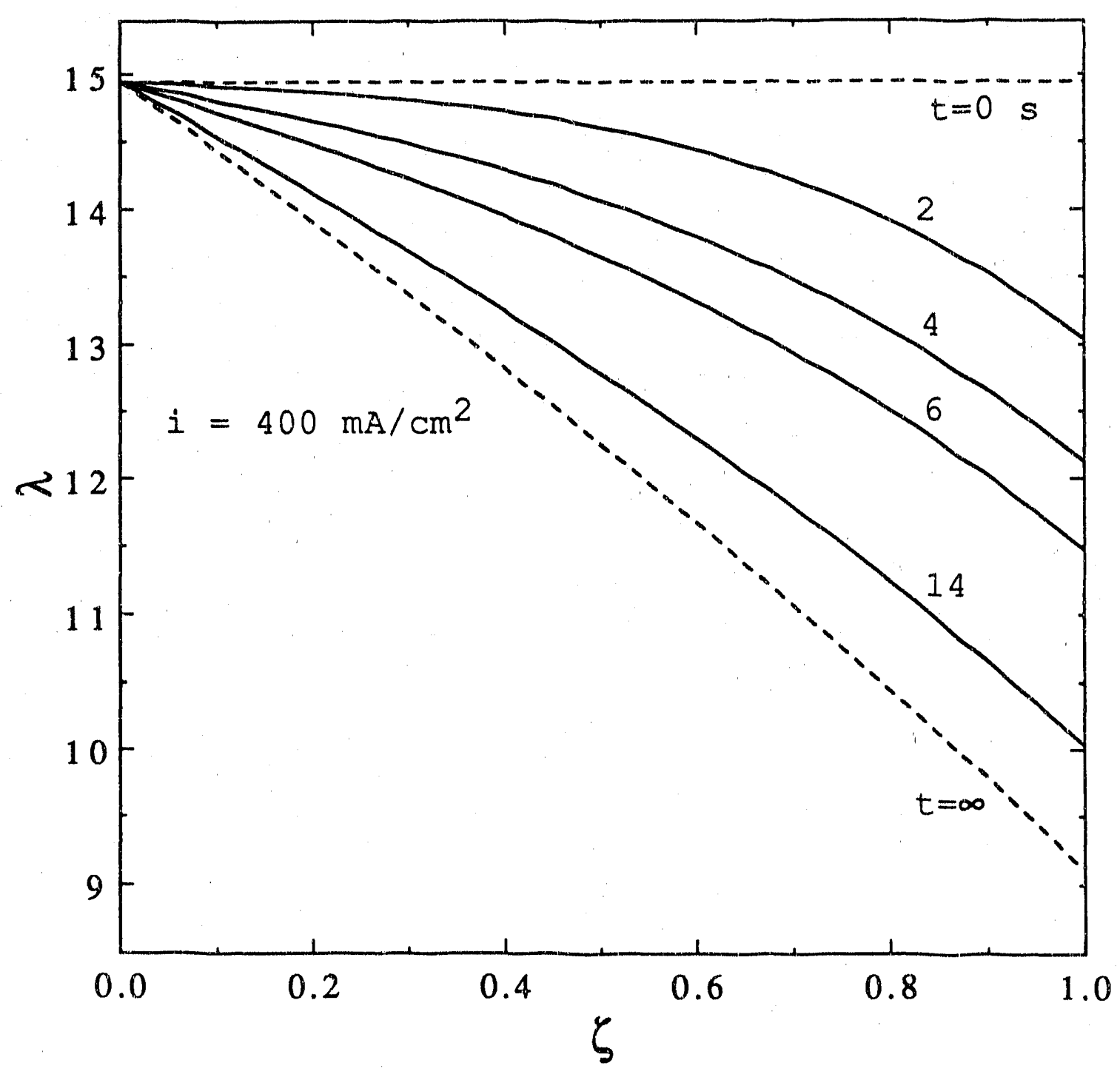

Figure 1. Water map across separator following a step change in current from 0 to $400 \mathrm{~mA} / \mathrm{cm}^{2}$. 


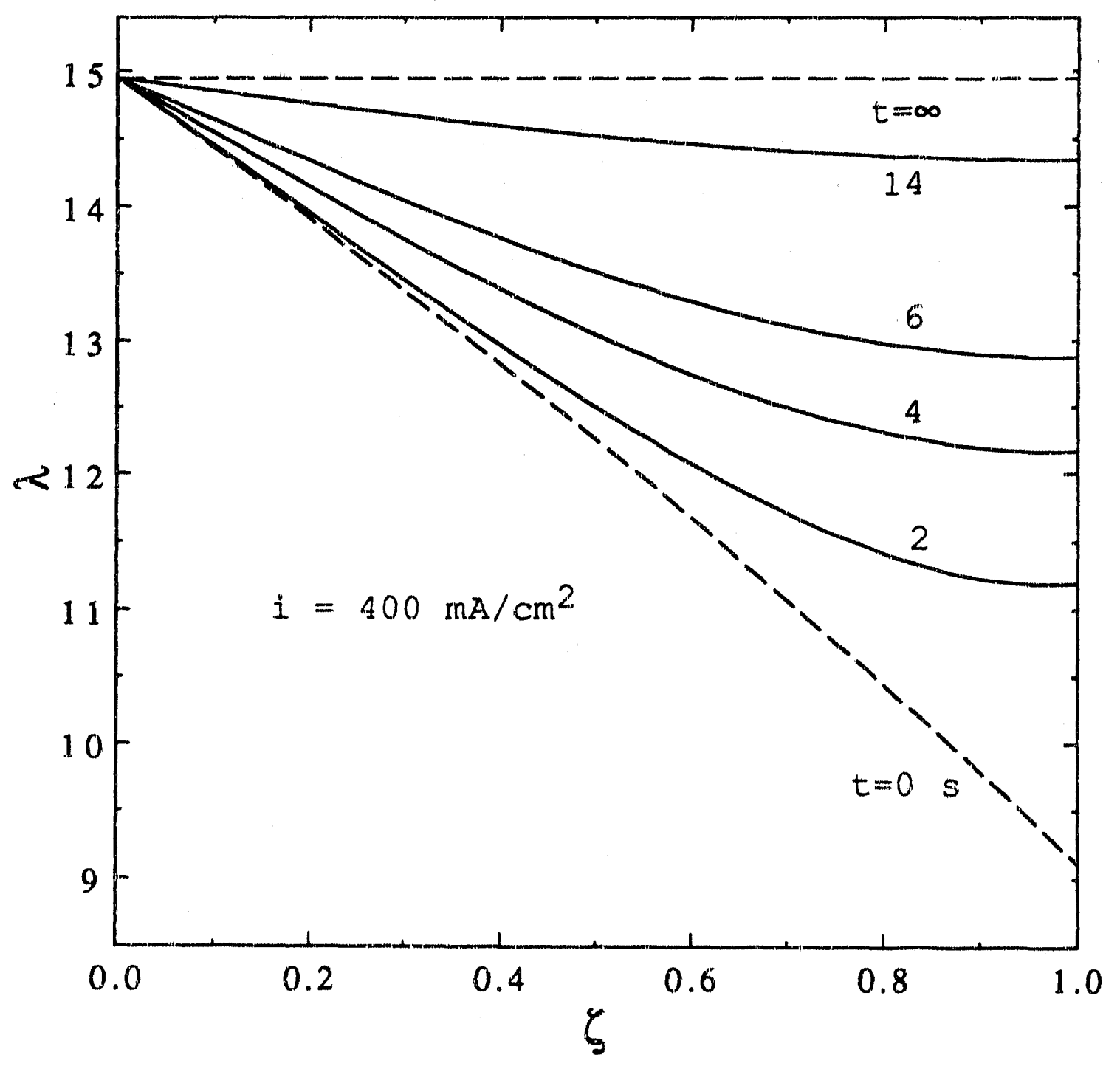

Figure 2. Water map across separator following a step change in current from 400 to $0 \mathrm{~mA} / \mathrm{cm}^{2}$. 
for higher current densities. One conclusion that, under these conditions, the fuel cell would be able to handie only short periods of high current density without dehydration of the membrane. In an operating fuel cell, after a step change in voltage, one observes a jump in current followed by a transient of about one minute. This may be associated with the transport of water in the membrane.

\section{Ifst of Symbols}

$\begin{array}{ll}c_{i} & \text { concentration of species } i, \mathrm{~mol} / \mathrm{m}^{3} \\ D & \text { diffusion coefficient, } \mathrm{cm}^{2} / \mathrm{s} \\ I & \text { Faraday's constant, } 96,487 \mathrm{C} / \mathrm{eq} \\ \ell & \text { current density } \mathrm{A} / \mathrm{m}^{2} \\ \mathbf{N}_{i} & \text { width of membrane, cm } \\ R & \text { molar flux of } i, \text { mol } / \mathrm{cm}^{2} \cdot \mathrm{s} \\ t & \text { universal gas constant, } 8.3143 \mathrm{~J} / \mathrm{mol} \cdot \mathrm{K} \\ T & \text { time, s } \\ y & \text { temperature, } \mathrm{K} \\ \alpha & \text { distance across membrane, } \mathrm{cm} \\ \zeta & \text { defined in equation } 5 \\ \kappa & \text { dimensionless distance } \\ H_{i} & \text { electrical conductivity, } \mathrm{S} / \mathrm{cm} \\ \xi & \text { electrochemical potential of species } i, \mathrm{~J} / \mathrm{mol} \\ \tau & \text { transport number of water } \\ & \text { time constant for diffusiori, s }\end{array}$


Subscripts

$\circ$

water

\section{References}

[1] Supramaniam Sxinivasan, "Fuel Cells for Extraterrestrial and Texrestrial Applications," J. Electrochem. Soc., 136, 41C (1989).

[2] T. E. Springer and I. D. Raistruck, "Electrical Impedance of a Pore Wall for the Flooded-Agglomerate Model of Porous Gas-Diffusion Electrodes," J. Electrochen. Soc., 136, 1594-1603 (1989).

[3] H. S. Carslaw and J. C. Jaeger, Conduction of Heat in Solids, Clarendon Press, Oxford (1957).

[4] C. F. Gerald, Applied Numerical Analysis, Addison Wesley, Reading, MA (1980). 
Appendix D

Electrochemical systems are often characterized with impedance methods. Because the physical phenomena can occur over different time scales, impedance spectroscopy may be used to obtain transport properties and kinetic data. Impedance techniques are discussed in [1] and [2]. We can measure the impedance of the system by introducing a small sinusoidal perturbation to the steady-state solution. The amplitude is small to ensure a linear response; and, because the system is linear, efther a small perturbation in cell voltage or current gives identical results.

Often, impedance data are interpreted with equivalent electrical circuits [3]. Our approach is to formulate the time-dependent conservation equations in terms of fundamental transport and kinetic phenomena and then solve these equations directly. Although we consider a specific system, our approach is general and could be applied to othex polymer electrolytes with little modification. Furthermore, this model should provide the basis for analysis of experimental results.

We investigated a thin membrane with an electrode at $y=0$. At $y=\ell$, the potential is set to zero, and the concentration is fixed. This is equivalent to a Nernst stagnant diffusion layer, for which an analytic solution is possible [4]. At open circuit, a small perturbation in the potential of the solution, $\Phi$, is introduced. The impedance is defined as 


$$
z=\frac{\bar{V}}{\tilde{I}}
$$

First we examine a systern with constant physical properties; then we consider the detailed model.

The transport equations for the solid polymer electrolyte were developed in section 7.2 . Substituting

$$
\begin{gathered}
c_{0}=\bar{c}_{0}+\operatorname{Re}\left\{\tilde{c}_{0} e^{j \omega t}\right\}, \\
\zeta=y / \ell, \quad \text { and } \quad \theta=\frac{\tilde{c}_{0}}{\bar{c}_{0}(0)},
\end{gathered}
$$

into equation 6 of section 7.2 yields

$$
\frac{l^{2}}{D} \theta j \omega=\frac{d^{2} \theta}{d \zeta^{2}} .
$$

With the boundary conditions

$$
\theta=0 \text { at } \zeta=1 \text { and } \theta=1 \text { at } \zeta=0 \text {, }
$$

the solution is well-known:

$$
\theta=\frac{\sinh \left(\sqrt{j \omega \ell^{2} / D(1-5)}\right)}{\sinh ^{\sqrt{j \omega \ell^{2} / D}}} .
$$

Substituting 


$$
i=\bar{i}+\operatorname{Re}\left\{\tilde{i} e^{j \omega t}\right\}
$$

and

$$
\mu_{0}-\bar{\mu}_{0}+\operatorname{Re}\left\{\tilde{\mu}_{0} e^{j \omega t}\right\}
$$

into equation 1 (section 7.2) and integrating gives

$$
\bar{i}=\frac{\kappa}{\ell} \Delta \tilde{\Phi}-\frac{\kappa \xi R T}{\ell F-\tilde{c}_{0}}\left(\tilde{c}_{0}(l)-\tilde{c}_{0}(0)\right) .
$$

This allows us to relate differences in the chemical potential of water to the current. At $5=0$, the flux of water is zero; therefore,

$$
\frac{\xi \tilde{i}}{F}=\frac{D}{\ell} \frac{d \theta}{d \zeta} \tilde{c}_{0}(0)
$$

Equation D-4 can be expressed as

$$
\bar{i}=\frac{\kappa}{\ell} \Delta \tilde{\Phi}-\frac{\kappa \xi^{2}}{\alpha F^{2}} \frac{\tilde{i}}{\theta^{\prime}(0)},
$$

where $\tilde{c}_{0}(0)$ is obtained from equation D-5. Equation D-3 is differentiated to yield

$$
-\frac{1}{\theta^{\prime}(0)}-\frac{\tanh ^{\sqrt{j \omega \ell^{2} / D}}}{\sqrt{j \omega \ell^{2} / D}}
$$

In the absence "f kinetic resistance, the imredance is therefore given by 


$$
z=\frac{\ell}{\kappa}\left(1+\frac{\kappa \xi^{2}}{\alpha F^{2}} \frac{\tanh ^{\sqrt{j \omega \ell^{2} / D}}}{\sqrt{j \omega \ell^{2} / D}}\right) .
$$

At high frequency, only the ohmic resistance,

$$
z_{r}=\frac{l}{t}
$$

is present. At low frequencies,

$$
z_{r}=\frac{\ell}{\kappa}\left(1+\frac{\kappa \xi^{2}}{\alpha F^{2}}\right)
$$

which also results from the steady-state solution of equations 1 and 2 (section 7.2), with the flux of water set to zero. With $\frac{\kappa \xi^{2}}{\alpha F^{2}}$ as a parameter, the real and imaginary paris of equation $D-8$ are shown in figure 1. With $D=1.0 \times 10^{-5} \mathrm{~cm}^{2} / \mathrm{s}$ and $\ell=0.02 \mathrm{~cm}$, the real and imaginary portions are plotted against frequency in figure 2.

Next, we wish to calculate the frequency response using the detailed model developed in the preceding chapters. Newman et al. [5] [6] have examined the impedance of a concentrated solution for a rotating-disk systern; we took a similar approach. Pollard and Comte recently presented work relating to the Impedance of solid-polymerelectrolytes [7].

Each of the dependent variables can be expressed as the sum of a steady-state part plus a small perturbation 


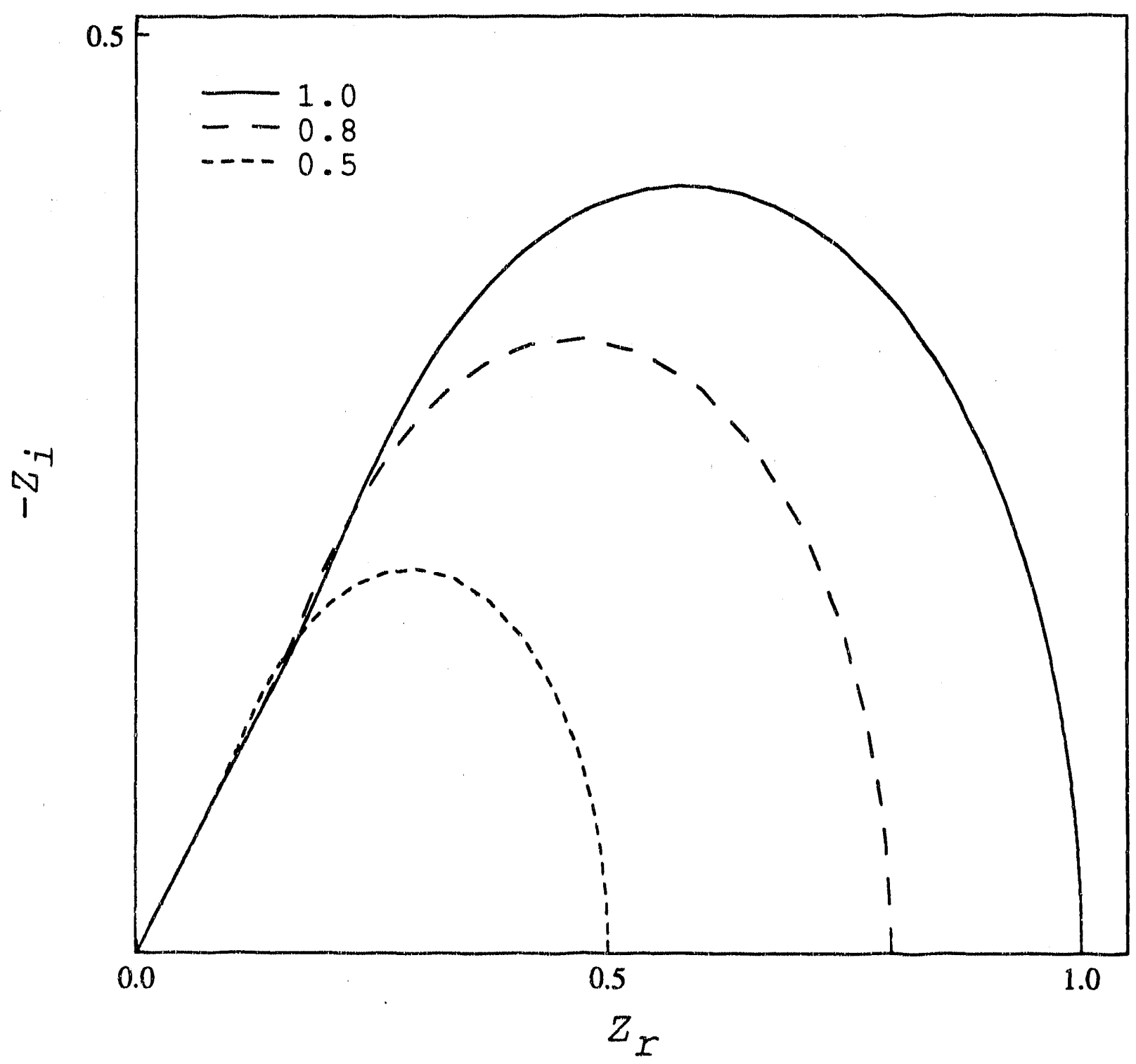

Figure $D-1$. Real and imaginary portions of impedance calculated from equation $D-8$. $\frac{\kappa \xi^{2}}{\alpha F^{2}}$ is a parameter. 


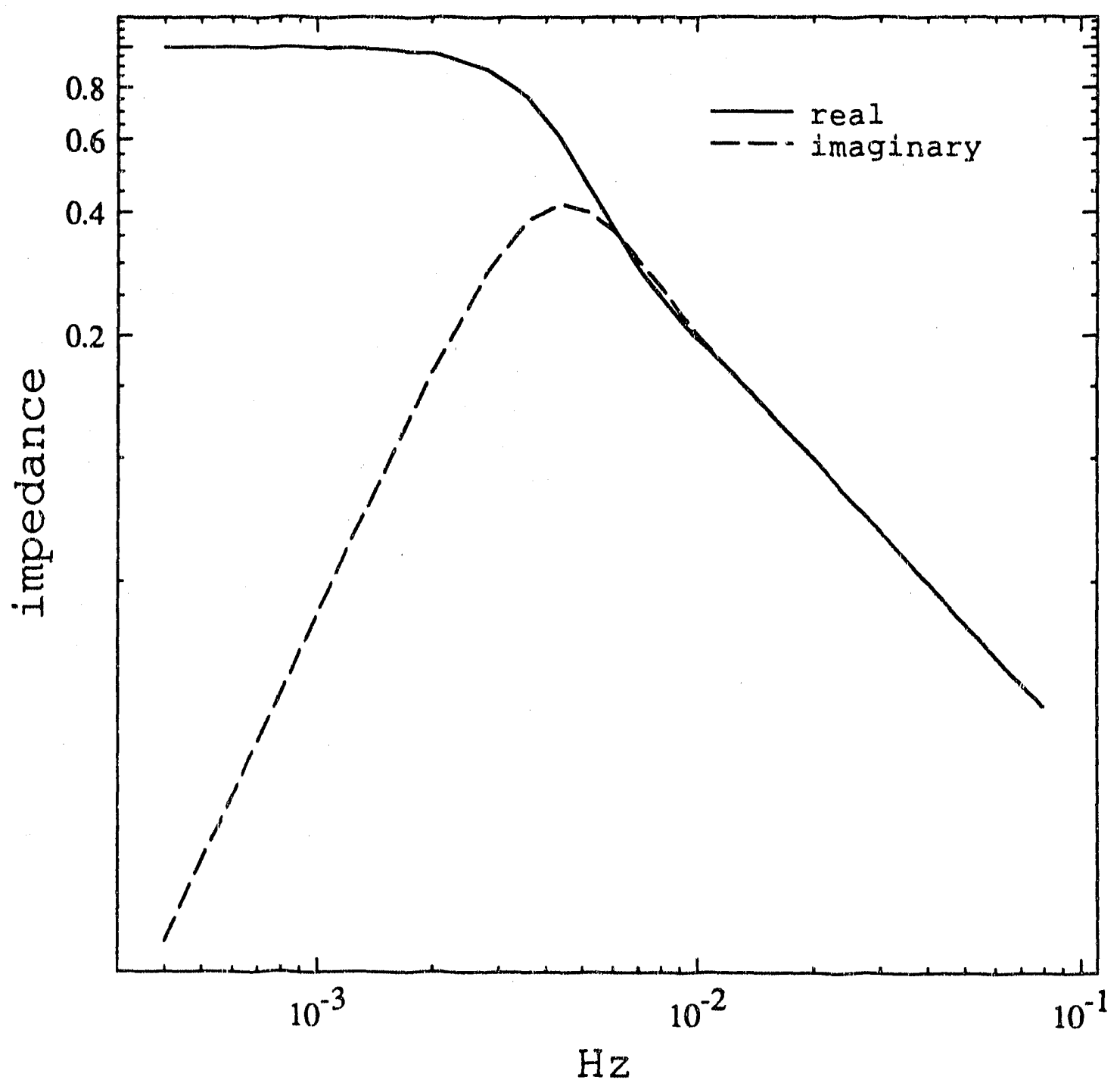

Figure D-2. Real and imaginary portions of impedance calculated from equation $D-8 . \quad D=1.0 \times 1 \sigma^{5} \mathrm{~cm}^{2} / \mathrm{s}$ and $\ell=0.02 \mathrm{~cm}$. 


$$
x_{i}=\bar{x}_{i}+\operatorname{Re}\left\{\tilde{x}_{i} e^{j \omega t}\right\}
$$

and

$$
\Phi-\bar{\Phi}+\operatorname{Re}\left\{\tilde{\Phi}_{e^{j \omega t}}\right\} .
$$

One substitutes these into the governing equations (see chapter 3 ). The steady-state solution will cancel out, leaving an equation for the real and imaginary parts of the variables. If there are $n$ equations and unknowns for the steady-state problem there will be $2 n$ equations for the transient case.

At $y=0$, the flux of each species to the electrode surface is described by Butler-Volmer kinetics. At $y-l$, the potential and composition are fixed. Because there is no capacitance in this model, the kinetic resistance can simply be added to the ohmic resistance.

Numerical results for the model are shown in figure 3 . These agree with the analytic results. Here, the electrode is reversible to hydrogen and blocks water. The parameters used are given in table D-1 and are typical values for a Nafion membrane. The computer program transient. for is 1 isted in appendix $E$.

Table D-1.

\begin{tabular}{|c|c|}
\hline$\frac{\ell}{\kappa}, \Omega-\mathrm{cm}^{2}$ & $\frac{\kappa \xi^{2}}{\alpha F^{2}}$ \\
\hline 0.1735 & 0.006974 \\
\hline
\end{tabular}




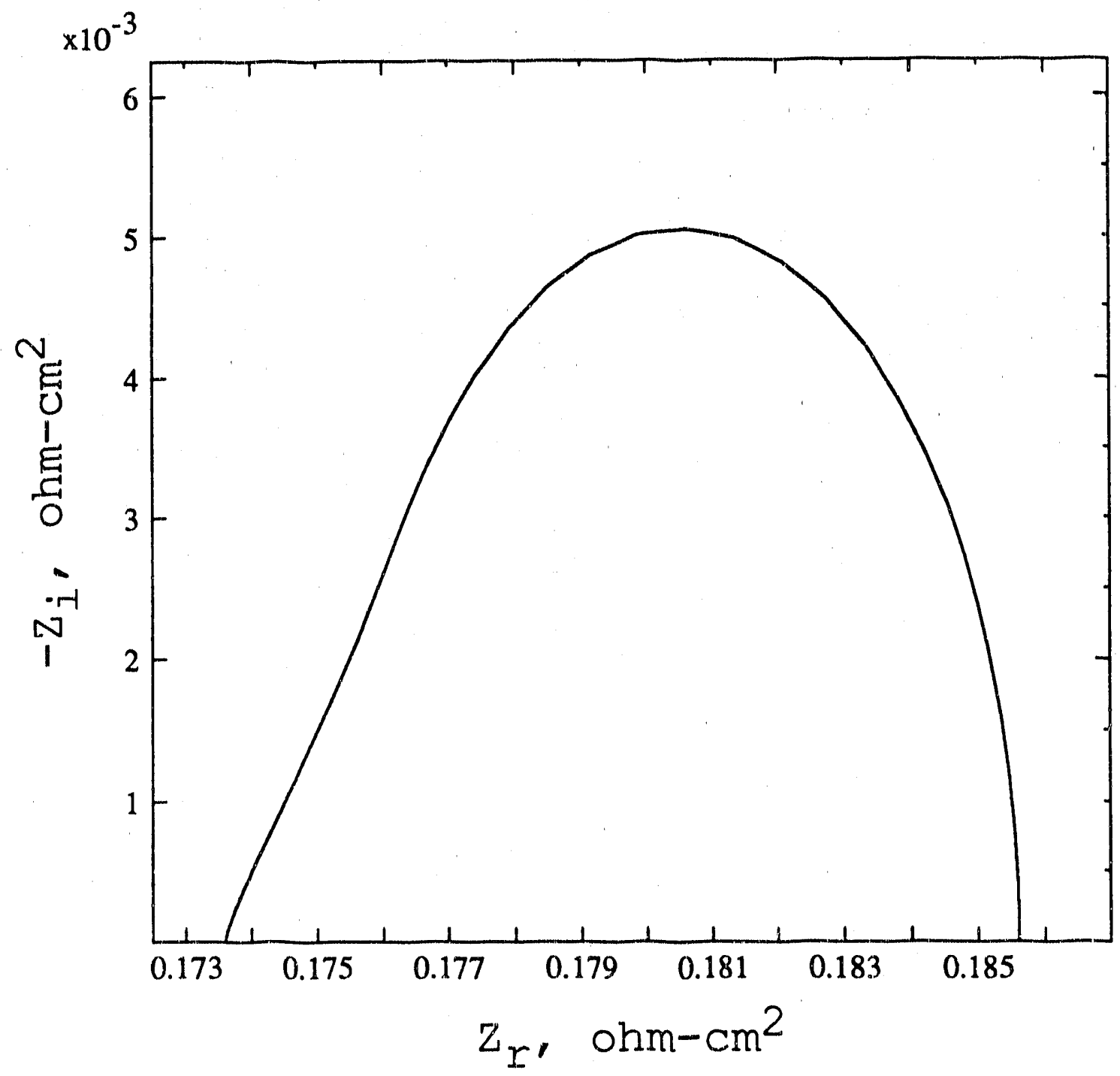

Figure D-3. Real and imaginary portions of impedance calculated from detailed model. 
While it is possible to obtain transport data from impedance spectroscopy, in the case of the Nafion membrane, this would require data at low frequencies. Furthermore, it would be difficult to find an electrode reversible to hydrogen but which blocks water under these conditions. In the preceding chapter we mentioned difficulties with the conductometric method. At high frequencies, only ohmic resistance is present; however, there was significant contact resistance between the membrane and electrode, which could be reduced only by applying pressure between the two blocks.

\section{List of Symbols}

$\begin{array}{ll}c_{i} & \text { concentration of species } i, \mathrm{~mol} / \mathrm{m}^{3} \\ D & \text { diffusion coefficient, } \mathrm{cm}^{2} / \mathrm{s} \\ F & \text { Faraday's constant, } 96,487 \mathrm{C} / \mathrm{eq} \\ 1 & \text { current density } \mathrm{A} / \mathrm{m}^{2} \\ \ell & \text { width of membrane, cm } \\ R & \text { universal gas constant, } 8.3143 \mathrm{~J} / \mathrm{mol} \cdot \mathrm{K} \\ t & \text { time, s } \\ T & \text { temperature, } \mathrm{K} \\ V & \text { overpotential or cell potential, } \mathrm{V} \\ x_{i} & \text { mole fraction of species i } \\ y & \text { distance across membrane, cm } \\ Z & \text { impedance } \\ \alpha & \text { defined in equation } 5 \text { (section } 7.2 \text { ) }\end{array}$




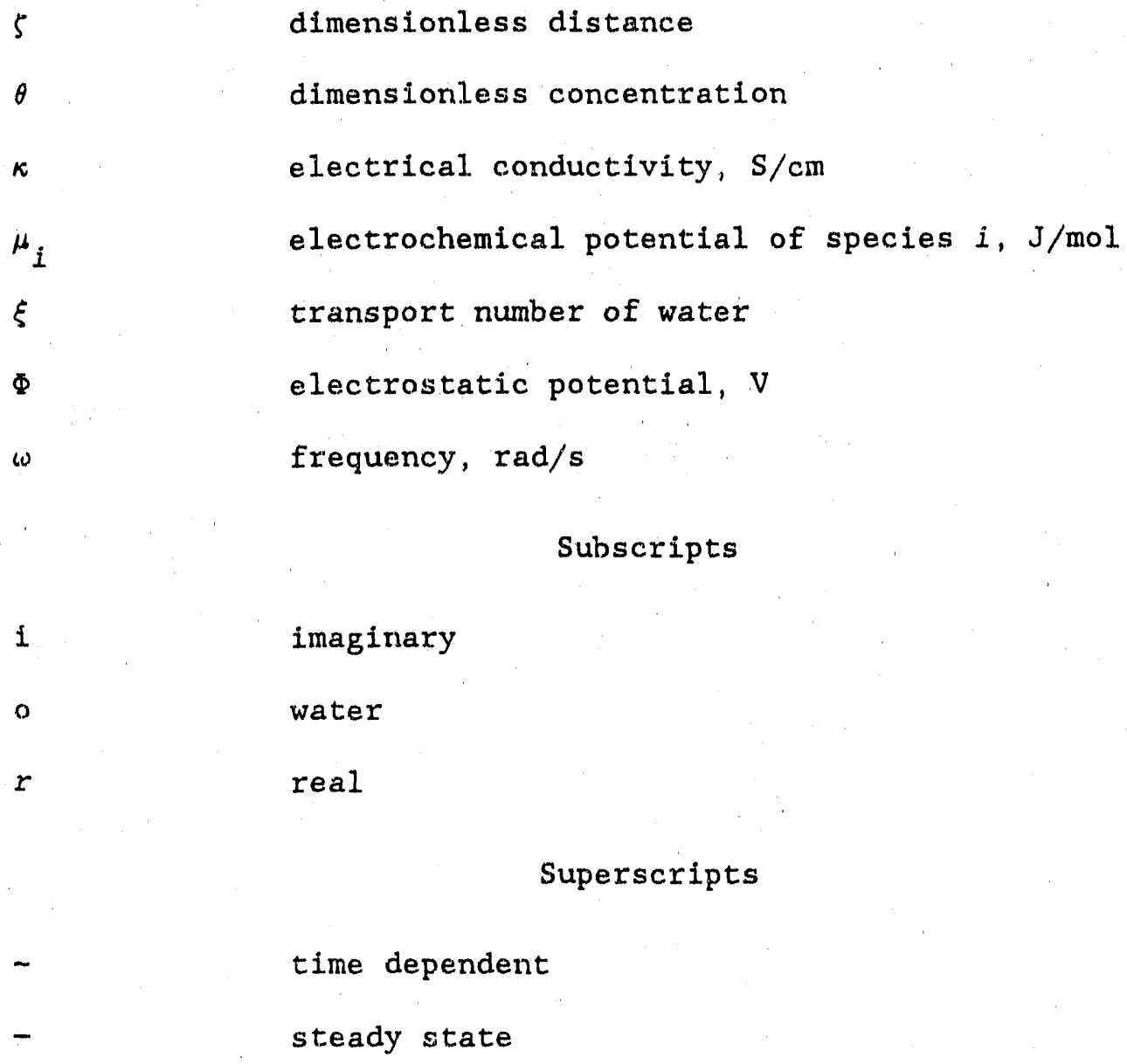

References

[1] Impedance Spectroscopy, edited by J. R. Macdonald, John Wiley \& Sons, New York (1987).

[2] A. J. Bard and L. R. Faulkner, Electrochemical Methods, John Wiley \& Sons, New York (1980).

[3] J. R. Macdonald, "Impedance/Admittance Response of a Binary Electrolyte," Electrochim. Acta, 37, 1007-1014 (1992).

[4] R. V. Homsy and J. Newman, "An Asymptotic Solution for the Warburg Impedance of a Rotatirg Disk Electrode," J. Electrochem. 
Soc., 121, 521-523 (1974).

[5]. B. Tribollet and J. Newman, "Impedance Model for a Concentrated Solution," J. Electrochem. Soc., 131, 2780-2785 (1984).

[6] A. K. Hauser and J. Newman, "The Effect of Schmidt Number on the Faradaic Impedance of the Dissolution of a Copper Rotating Disk," J. Electrochem. Soc., 136, 2896-2902 (1989).

[7] Richard Pollard and Thierry Comte, "Determination of Transport Properties for Solid Electrolytes from the Impedance of Thin Layer Cells," J. Electrochem. Soc., 136, 3734-3748 (1989). 
Appendix E

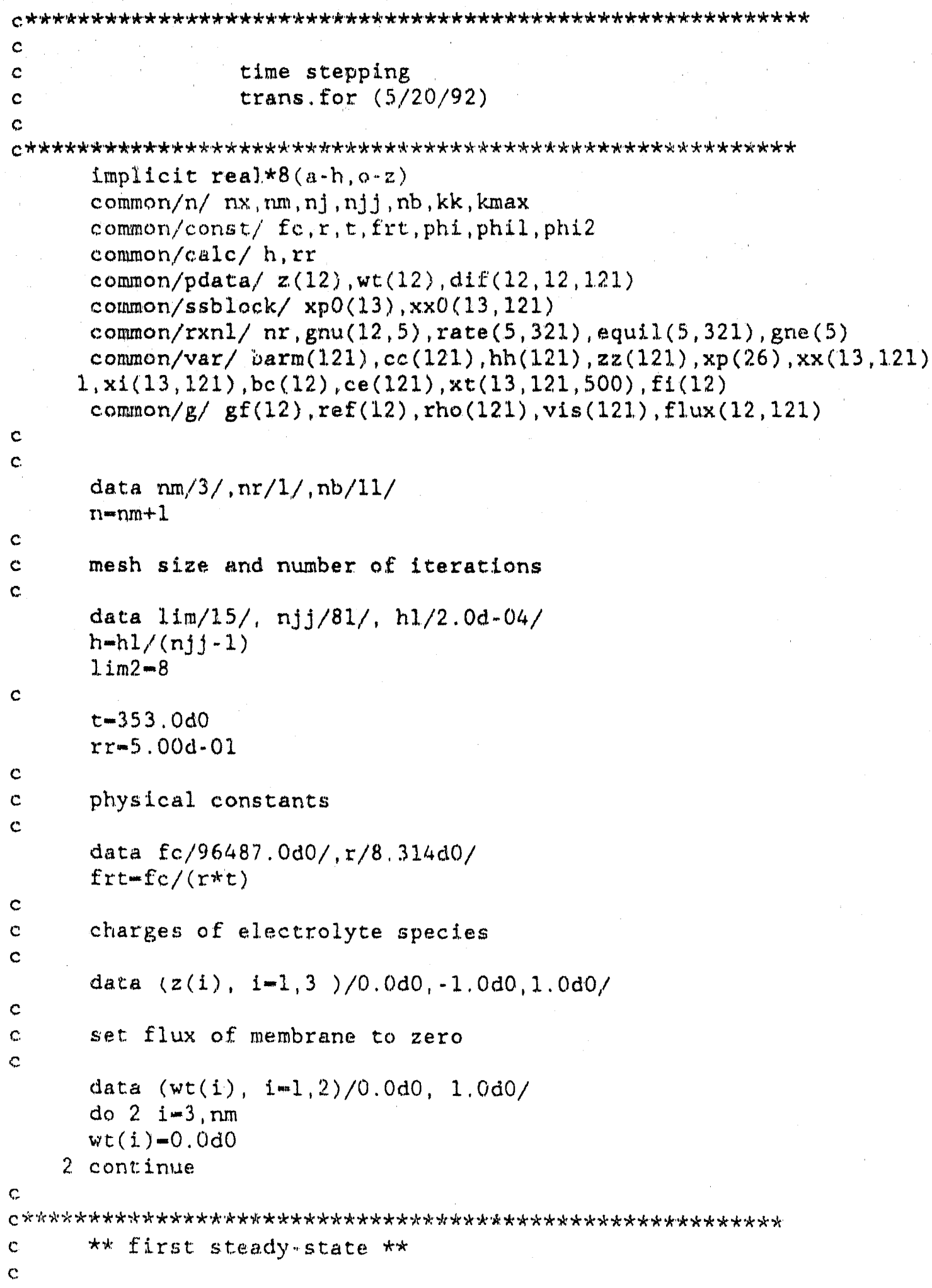




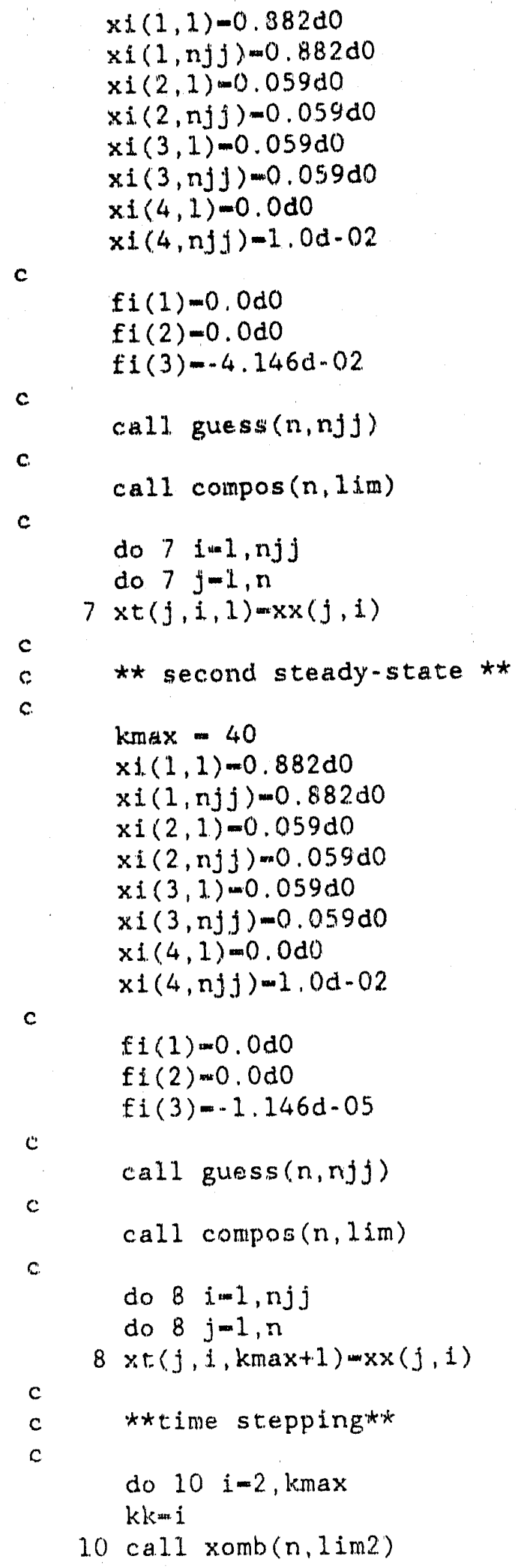




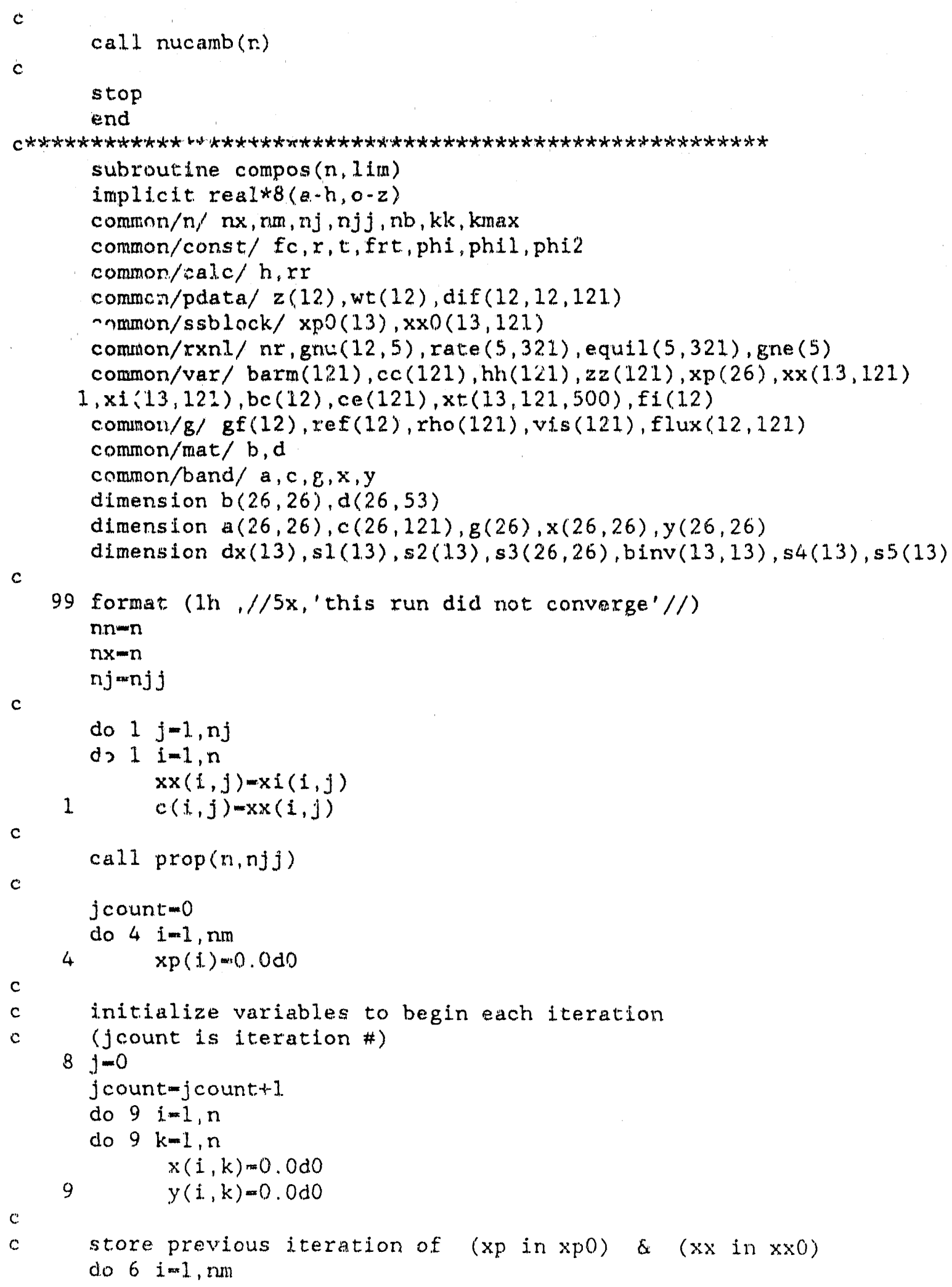




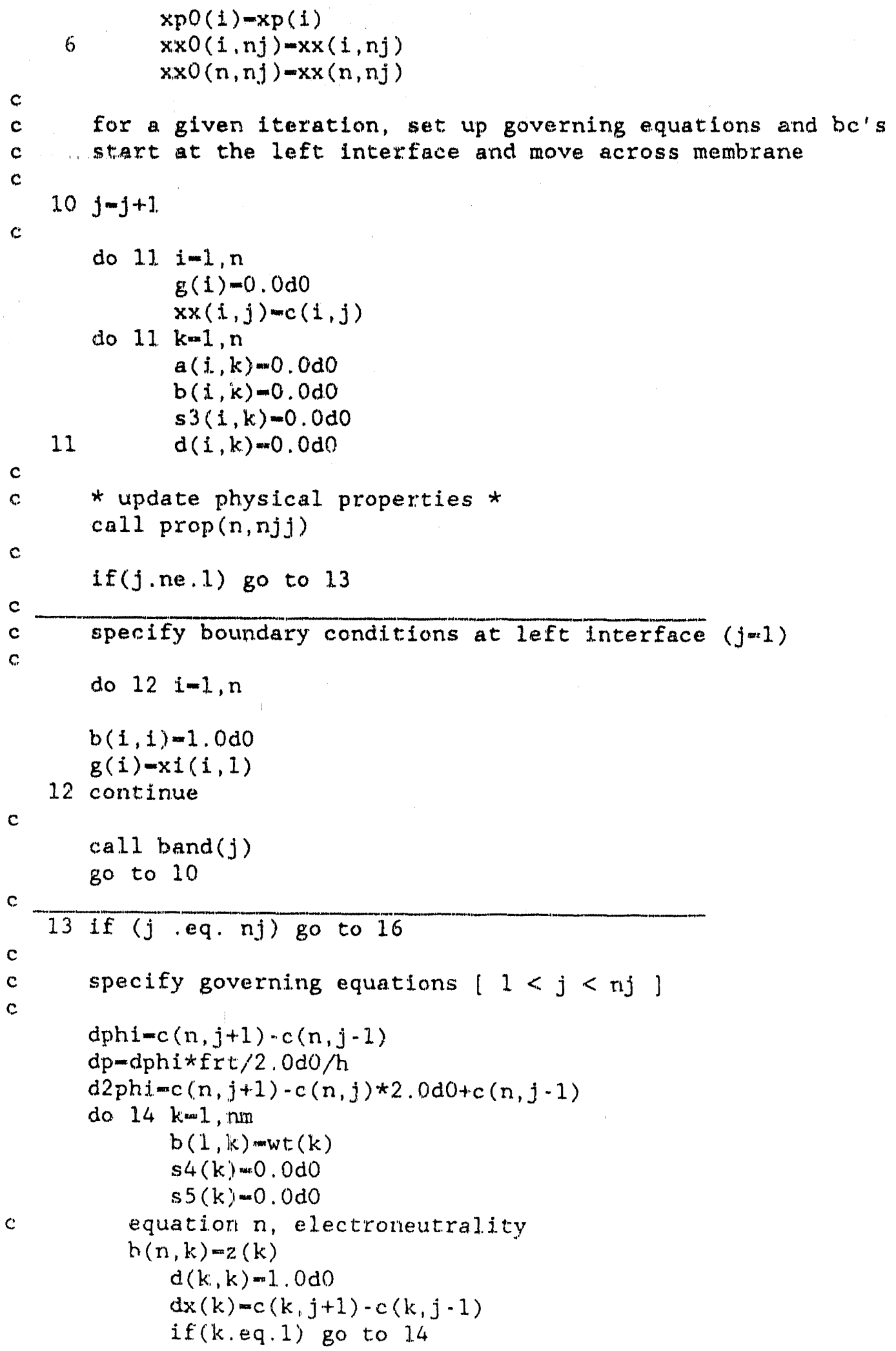


18

$$
\text { do } \begin{array}{rl}
18 & 1-1, n m \\
& i f(i, e q, k) \text { go to } 18 \\
& b(k, i)=c(k, j) / d i f(i, k, j) \\
& b(k, k)-b(k, k)-c(i, j) / d i f(i, k, j)
\end{array}
$$

14 continue

$$
\begin{aligned}
& \text { continue } \\
& d(k, n)-(d x(k)+z(k) * f r t * c(k, j) * d p h i) / 2.0 d 0 / h
\end{aligned}
$$

call matinv( $\mathrm{nm}, \mathrm{n}$, determ)

do $201-1, \mathrm{~nm}$

$\operatorname{fIux}(i, j) m d(i, n)$

do $19 \mathrm{k}=1, \mathrm{~nm}$

$$
b i \operatorname{riv}(i, k)-d(i, k)
$$

19

$$
b(i, k)=0.0 d 0
$$

$b(i, n)=0.0 d 0$

$d(i, n)=0.0 d 0$

c

equation 1 , the sum of mole fractions equals 1.0

$20 \mathrm{~b}(1, i)=1.0 \mathrm{~d} 0$

do $22 \quad i=1, \mathrm{~nm}$

$\operatorname{si}(i)=0.0 \mathrm{do}$

$s 2(i)=0.0 \mathrm{~d} 0$

do $22 \mathrm{~km}, \mathrm{~nm}$

if $(k . e q . i)$ go to 22

$a 2=f \operatorname{lux}(k, j) / \mathrm{dif}(i, k, j)$

$s 4(i)-s 4(i)+f l u x(k, j) / \operatorname{dif}(i, k, j+1)$

s5(i) $-s 5(i)+\operatorname{flux}(k, j) / \operatorname{dif}(i, k, j-1)$

$a 1=(c(k, j+1) / \operatorname{dif}(i, k, j+1)-c(k, j-1) / \operatorname{dif}(i, k, j-1)) / 2.0 \mathrm{~d} 0 / \mathrm{h}$

$s 1(i)-s i(i)+a l$

$s 2(i)-s 2(i)+a 2$

21

do $21 \mathrm{l}-1, \mathrm{~nm}$

22 continue

$$
\mathrm{s} 3(1, k)-s 3(1, k)+b i n v(i, 1) * a 1
$$

do $15 \mathrm{i}=2, \mathrm{~nm}$

$g(i)-g(i)-f \operatorname{lux}(i, j) * s l(i)$

$s 4(i)=s 4(i) / 2.0 \mathrm{~d} 0 / \mathrm{h}$

$\mathrm{s} 5(i)-\mathrm{s} 5(\mathrm{i}) / 2.0 \mathrm{dO} / \mathrm{h}$

do $27 \mathrm{k}=1, \mathrm{~nm}$

s $3(k, i)-s 3(k, i)-b i n v(i, k) * s 1(i)$

if $(k . \in q .1)$ go to 5

$d(i, k)=d(i, k)+s 3(k, i)$

$d(i, n)-d(i, n)+z(k) * c(k, j) * \operatorname{frt} * s 3(k, i) / 2.0 d 0 / h$

$a(j, n)-a(i, n)-z(k) * c(k, j) * f_{r} t * s 3(k, i) / 2.0 d 0 / h$

5

27

continue if $(k$, eq.i) go to 27

do $24 k=1$, nun

$d(i, k)=d(i, k) / 2.0 \mathrm{~d} 0 / \mathrm{h}$

$a(i, k)=-d(i, k)$

do $23 \quad 1-2, \mathrm{~nm}$

23 if $(k . n e .1) b(i, k)-b(i, k)-s 2(k) * s 3(k, i)+s 3(k, i) * z(k) * d p$ 


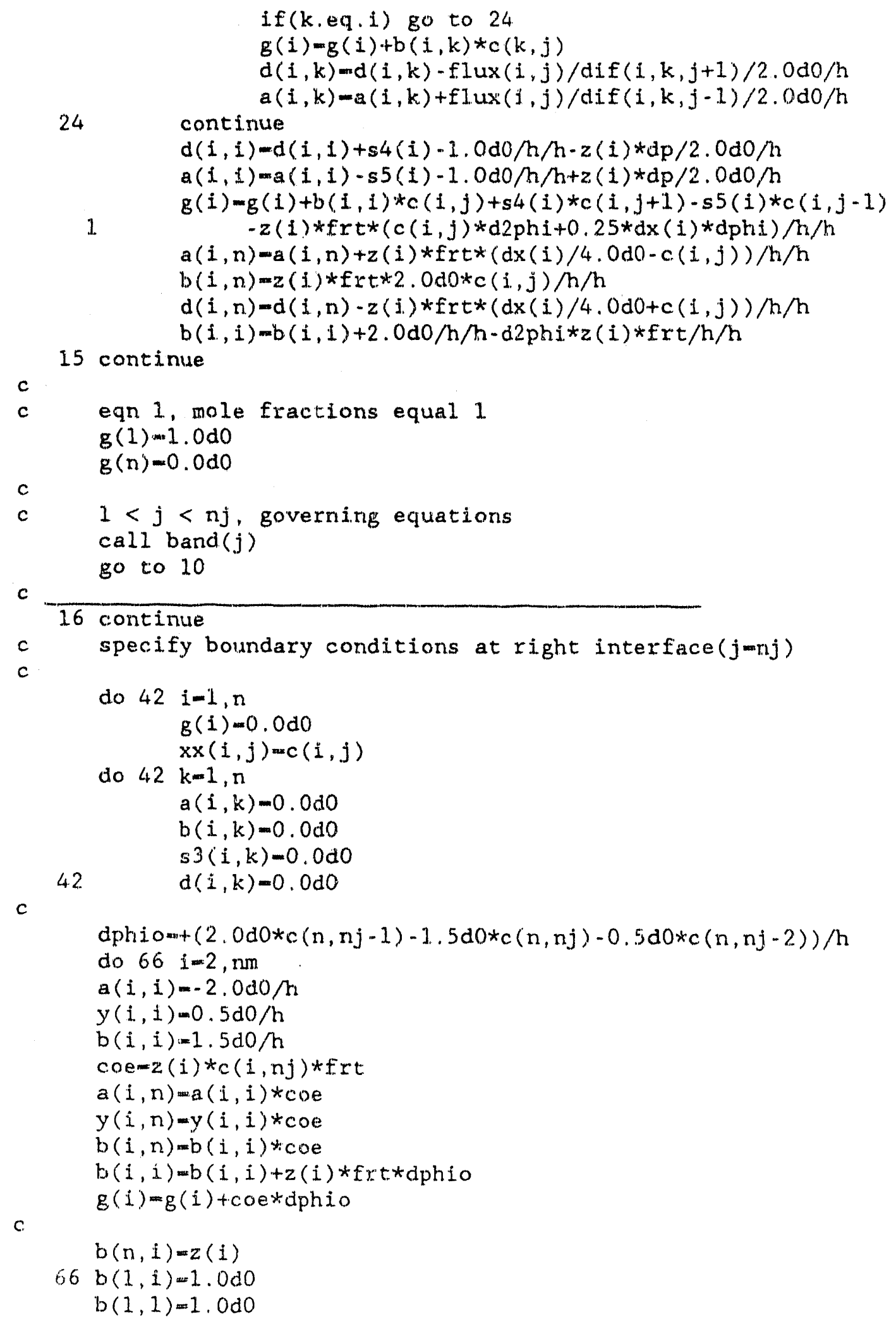




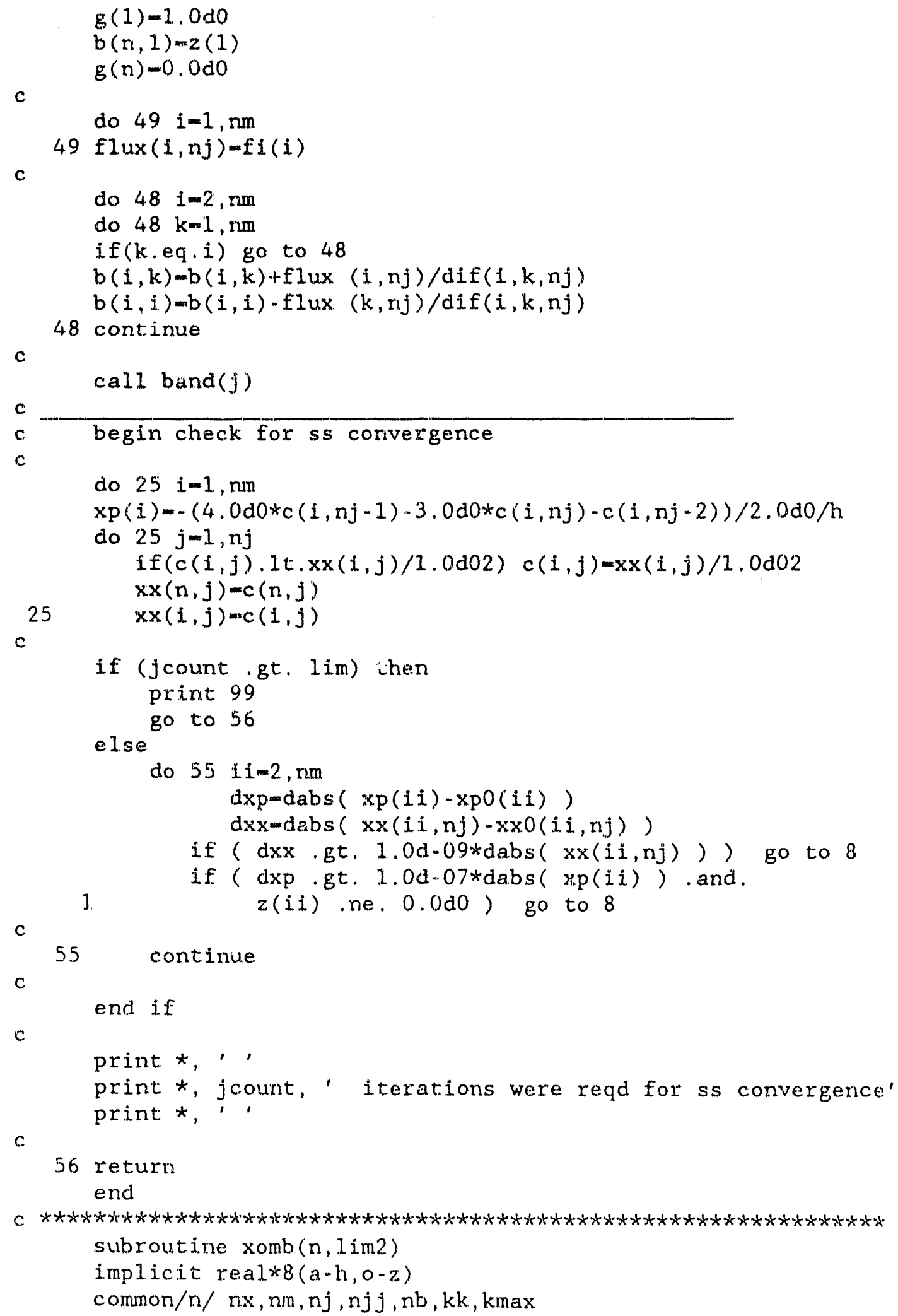


common/const/ fc, $r, t, f r t, p h i, p h i l, p h i 2$

common/calc/ h, rr

common/pdata/ $z(12), w t(12), \operatorname{dif}(12,12,121)$

common/ssblock/ $\mathrm{xpO}(13), \mathrm{x} \times 0(1,3,121)$

common/var/ barm(121), cc(121), hh(121), zz(121), xp (26), $x x(13,121)$

$1, x i(13,121), b c(12), c e(121), x t(13,121,500)$, fi $(12)$

common/g/ $g f(12), \operatorname{ref}(12)$, rho(121), vis(121), flux $(12,121)$

common/mat/ b, d

common/band/ $a, c, g, x, y$

dimension $b(26,26), d(26,53)$

dimension $a(26,26), c(26,121), g(26), x(26,26), y(26,26)$

dimension $\mathrm{dx}(13), \mathrm{si}(13), \mathrm{s} 2(13), \mathrm{s} 3(26,26), \mathrm{binv}(13,13), \mathrm{s} 4(13), \mathrm{s} 5(13)$

$c$

99 format ( $1 \mathrm{~h}, / / 5 \mathrm{x}$, 'this run did not converge'//)

$n n=n$

$n x-n$

c

$n j-n j j$

do $I i=1, n$

$x \times(i, 1)-x i(i, 1)$

$x x(i, n j)=x t(i, n j, k k-1)$

$c(i, 1)-x i(i, 1)$

$c(i, n j)=x t(j, n j, k k-1)$

do $1 \quad j=2, n j-1$

1 $c(i, j)=x t(i, j, k k-j)$

c

call $\operatorname{prop}(n, n j j)$

c

jcount $=0$

do 4 i $=1, n m$

c

$4 \mathrm{xp}(\mathrm{i})=0.0 \mathrm{~d} 0$

c

$8 j=0$

initialize variables to begin each iteration

jcountw $j$ count +1

do $9 i=1, n$

do $9 \mathrm{k}=1, \mathrm{n}$

9

$x(i, k)=0.0 \mathrm{do}$

$c$

$c$

store previous iteration of ( $x p$ in $x p 0) \&(x x$ in $x x 0)$

do $6 i=1$, nin

$\mathrm{xpO}(i)=\mathrm{xp}(i)$

$6 \times x O(i, n j)=x \times(i, n j)$

$x \times 0(n, n j)=x x(n, n j)$

c

c.

for a given iteration, set up governing equations and $b c^{\prime} s$

start at the left interface and move across membrane 
11

c

c

c

c

c

c

c

c

c

c

c

C

c.

$10 j=j+1$

do $11 \mathrm{i}=1, \mathrm{n}$

$g(i)=0.0 \mathrm{~d} 0$

do $11 k=1, n$ $x x(i, j)-c(i, j)$

$a(i, k)=0.0 \mathrm{~d} 0$

$b(i, k)=0.0 \mathrm{~d} 0$

s3 $(i, k)=0.0 \mathrm{~d} 0$ $\mathrm{d}(i, k)=0.0 \mathrm{~d} 0$

* update physical properties *

call $\operatorname{prop}(n, n j j)$

if (j,ne.1) go to 13

specify boundary conditions at left interface $(j=1)$

do $12 i=1, n$

$b(i, i)=1.0 \mathrm{~d} 0$

$g(i)=x i(i, 1)$

12 continue

call band $(j)$

go to 10

13 if $(j$.eq. $n j)$ go to 16

specify governing equations $[1<j<n j]$

$\operatorname{dphi}=c(n, j+1)-c(n, j-1)$

$\mathrm{dp}=\mathrm{dph} i * \mathrm{frt} / 2.0 \mathrm{~d} 0 / \mathrm{h}$

$d 2 p h i=c(n, j+1)-c(n, j) * 2.0 d 0+c(n, j-1)$

do $14 \mathrm{k}=1, \mathrm{~nm}$

$b(1, k)=w t(k)$

$s 4(k)=0.0 \mathrm{~d} 0$

$s 5(k)=0.0 \mathrm{~d} 0$

equation $n$, electroneutrality

$b(n, k)=z(k)$

$d(k, k)=1.0 d 0$

$d x(k)=c(k, j+1)-c(k, j-1)$

if $(k . e q .1)$ go to 14

do $18 i=1, \mathrm{~nm}$

if(i.eq.k) go to 18

$b(k, i)=c(k, j) / d i f(i, k, j)$

$b(k, k)=b(k, k)-c(i, j) / d i f(i, k, j)$

18

continue

$d(k, n)=(d x(k)+z(k) * \operatorname{frt} * c(k, j) * d p h i) / 2.0 d 0 / h$ 


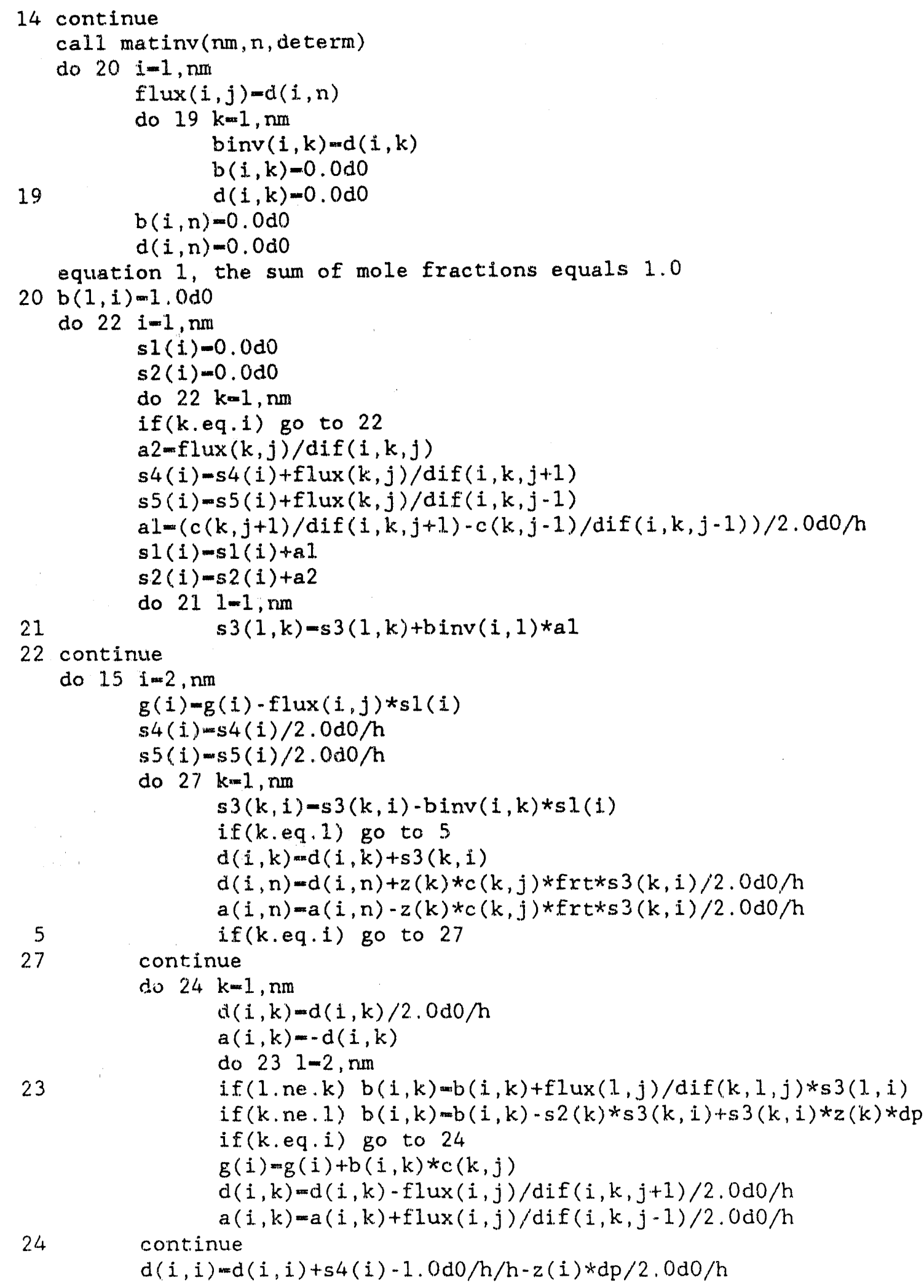




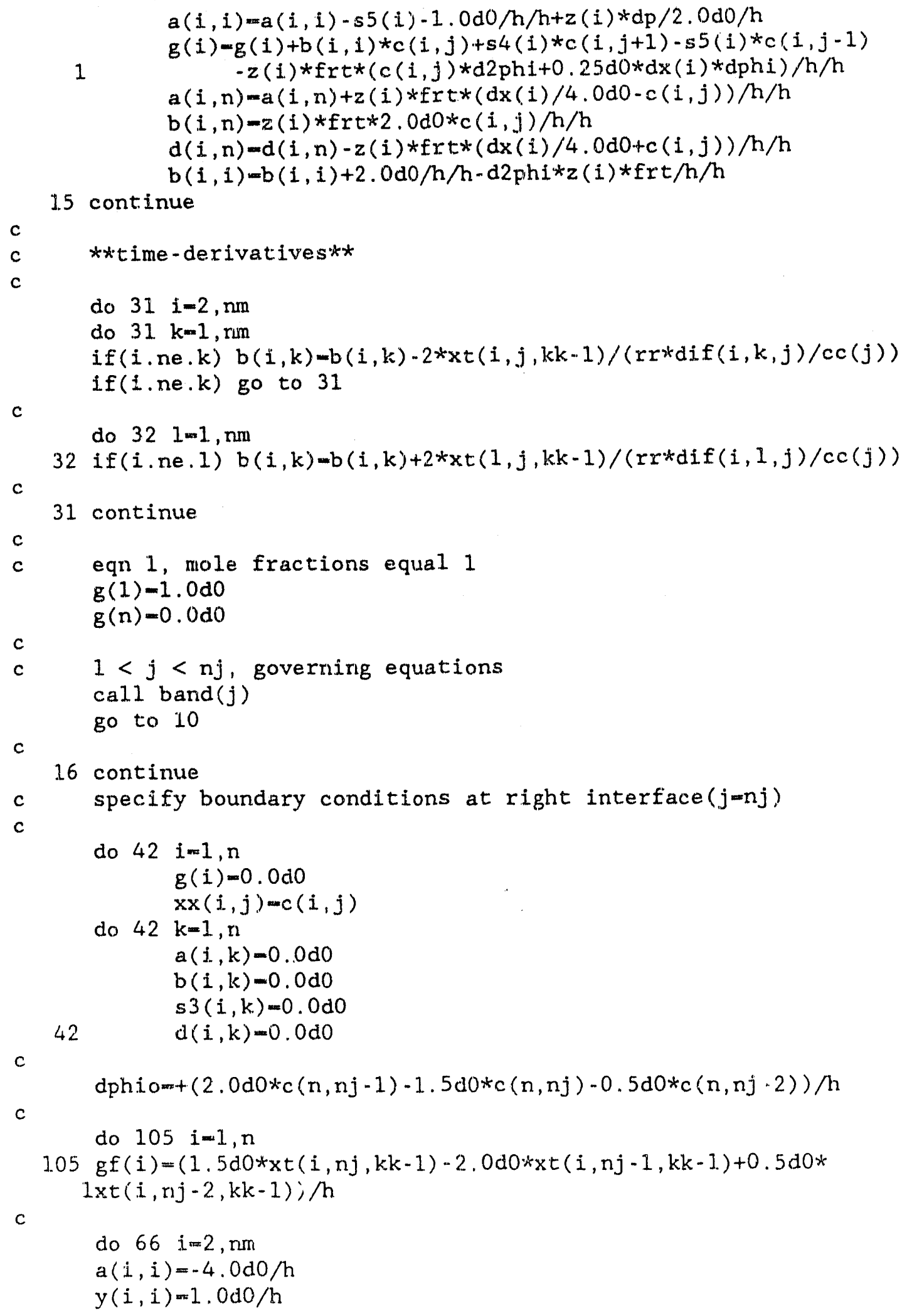


$b(i, i)-3.0 \mathrm{~d} 0 / \mathrm{h}$

$\operatorname{coe}-z(i) * c(i, n j) * f r t$

$a(i, n)=a(i, i) * c o e$

$y(i, n)-y(i, i) * c o e$

$b(i, n)=b(i, i) * c o e$

$b(i, i)-b(i, i)-2.0 d 0 * z(i) * f r t * d p h i o-2.0 d 0 * z(i) * f r t * g f(n)$

$g(i)=g(i)-2.0 d 0 * z(i) * f r t * x t(i, n j, k k-1) * \operatorname{dphio}-z(i) * f r t *$

$\operatorname{Ixt}(i, n j, k k-1) * g f(n)+g f(i)$

c

$b(n, i)-z(i)$

$66 \mathrm{~b}(1, i)=1.0 \mathrm{~d} 0$

$\mathrm{b}(1,1)=1.0 \mathrm{~d} 0$

$g(1)=1.0 \mathrm{~d} 0$

$b(n, 1)-z(1)$

$g(n)=0.0 \mathrm{~d} 0$

do $49 i=1, \mathrm{~nm}$

$49 \operatorname{flux}(i, n j)=f i(1)$

c

do $4 \hat{6} \quad \mathrm{i}=2, \mathrm{~nm}$

do $48 \mathrm{k}=1, \mathrm{~nm}$

if $(k$. eq. 1$)$ go to 48

$b(i, k)=b(i, k)+2.0 d 0 * f l u x(i, n j) / d i f(i, k, n j)$

$b(i, i)-b(i, i)-2.0 d 0 * f l u x(k, n j) / d i f(i, k, n j)$

48 continue

c

do $106 i=2, \mathrm{~nm}$

do $106 \mathrm{~km}=1, \mathrm{~nm}$

if (k.eq.i) go to 106

$g(i)=g(i)+.x t(k, n j, k k-1) * f l u x(i, n j) / d i f(i, k, n j)-$

$\operatorname{lxt}(i, n j, k k-1) \div f l u x(k, n j) / \operatorname{dif}(i, k, n j)$

106 continue

c

call band $(j)$

c

c begin check for convergence

$c$

do $25 i=1, \mathrm{~nm}$

$\operatorname{xp}(i)=-\left(4.0 \mathrm{dO} *_{c}(i, n j-1)-3.0 \mathrm{~d} 0 *_{c}(i, n j)-c(i, n j-2)\right) / 2.0 \mathrm{~d} 0 / \mathrm{h}$

do $25 j=1, n j$

if $(c(i, j) \cdot 1 t \cdot x x(i, j) / 1.0 d 02) c(i, j)=x x(i, j) / 1.0 d 02$

$x x(n, j)-c(n, j)$

$25 \quad \mathrm{xx}(i, j)=c(i, j)$

c

if (jcount gt. $\lim 2$ ) then

print 99

go to 57

else

do 55 ii $=2, \mathrm{~nm}$

dxp=dabs ( $x p(i i)-x p 0(i i))$ 


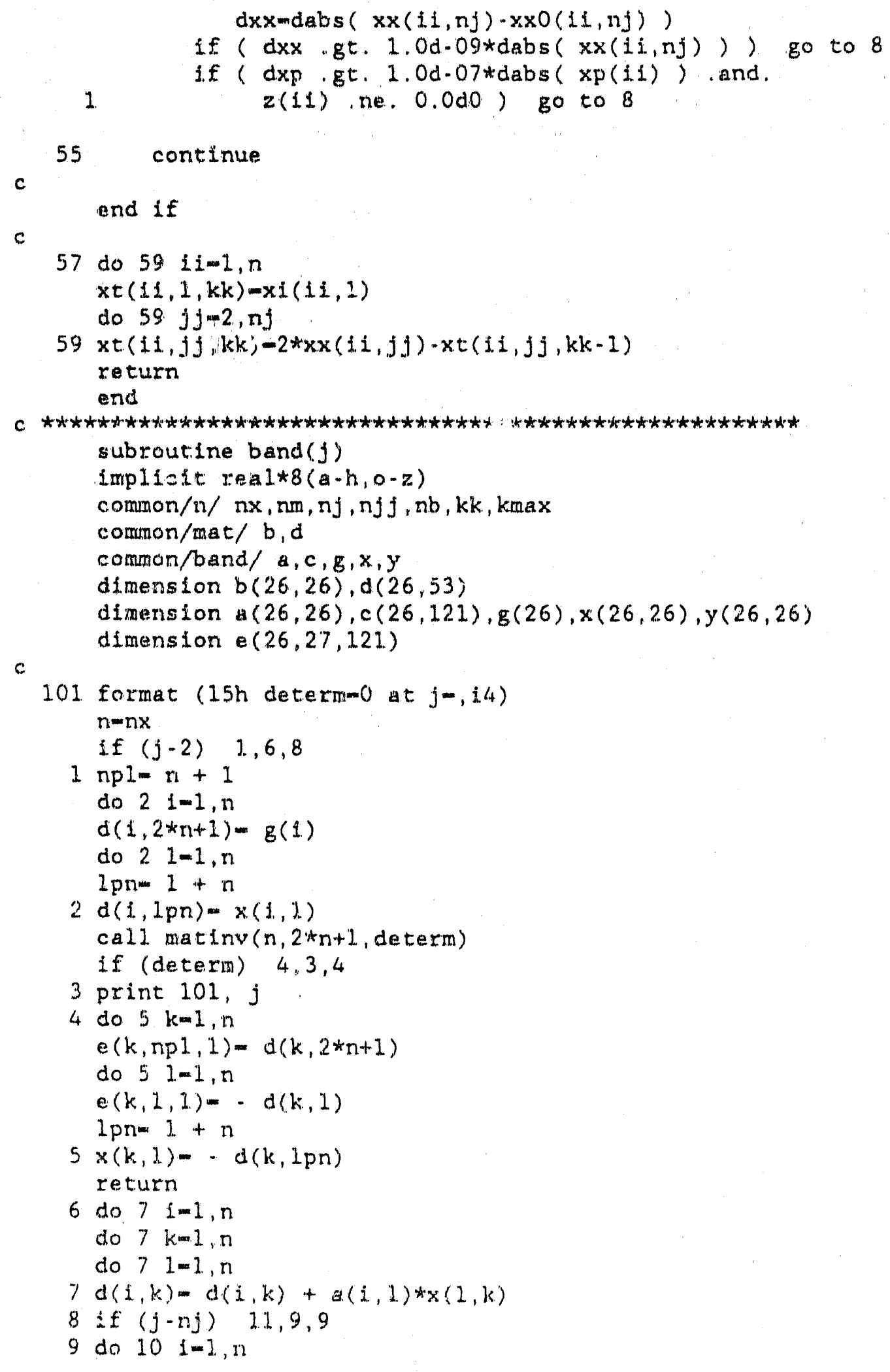




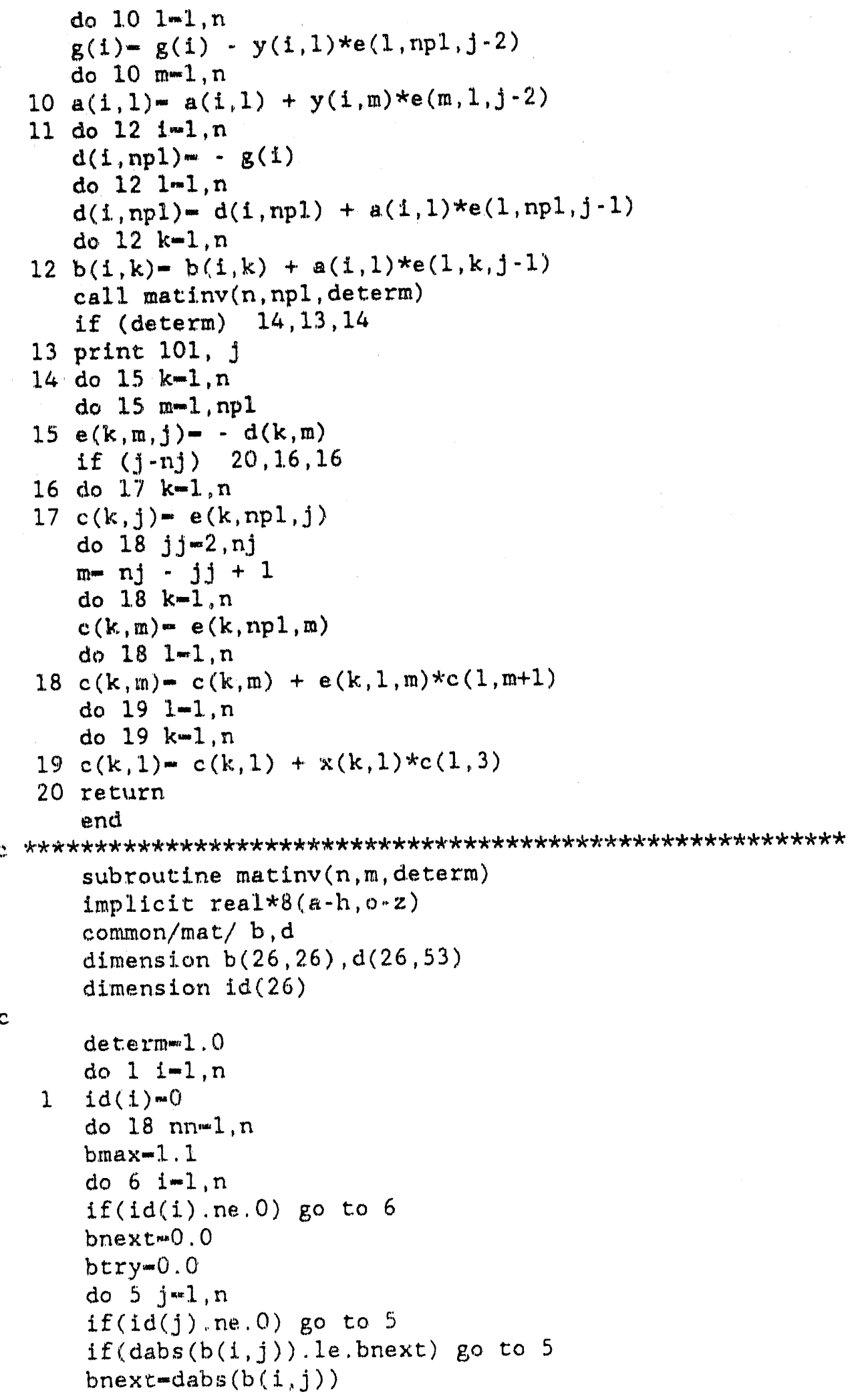




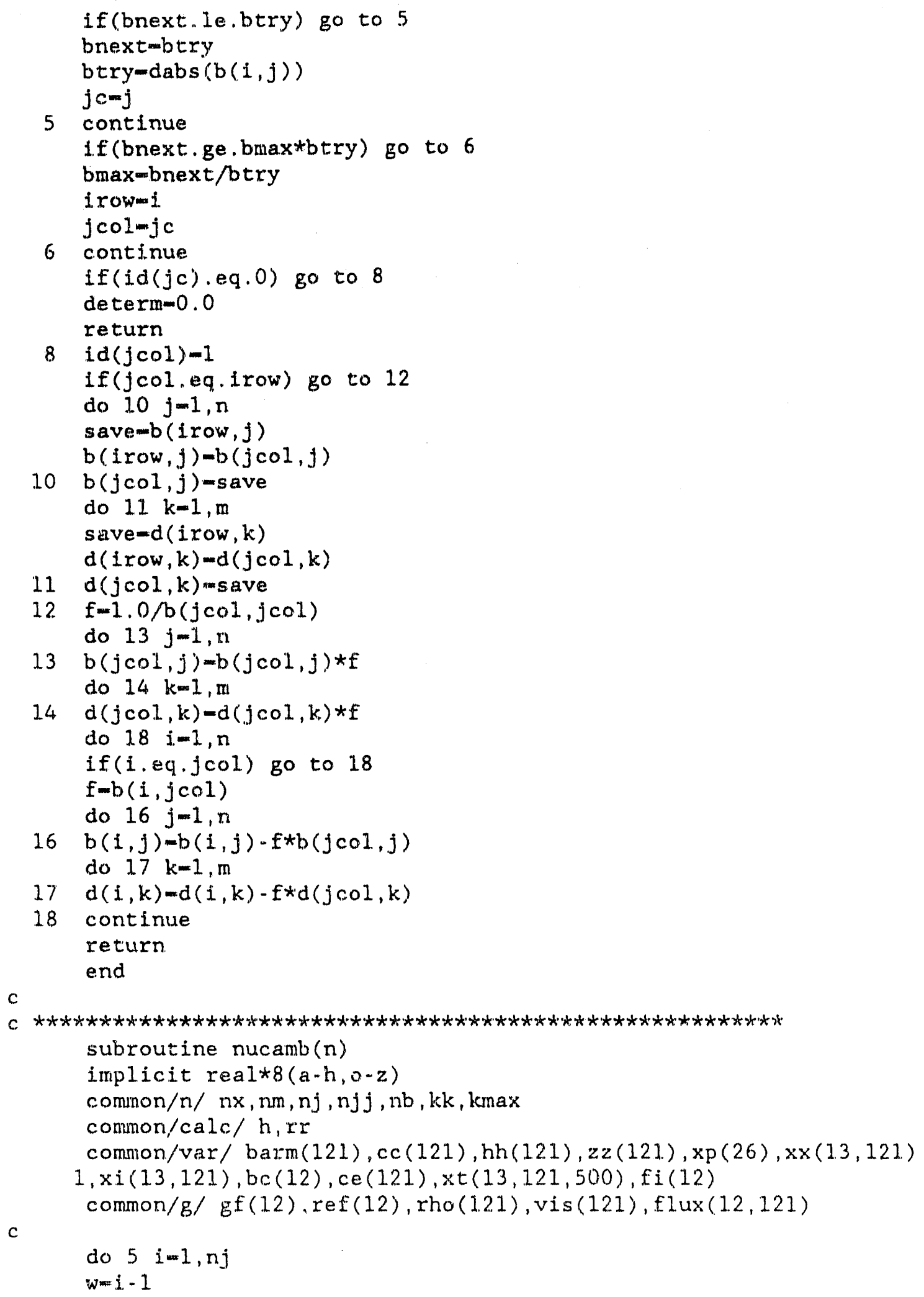




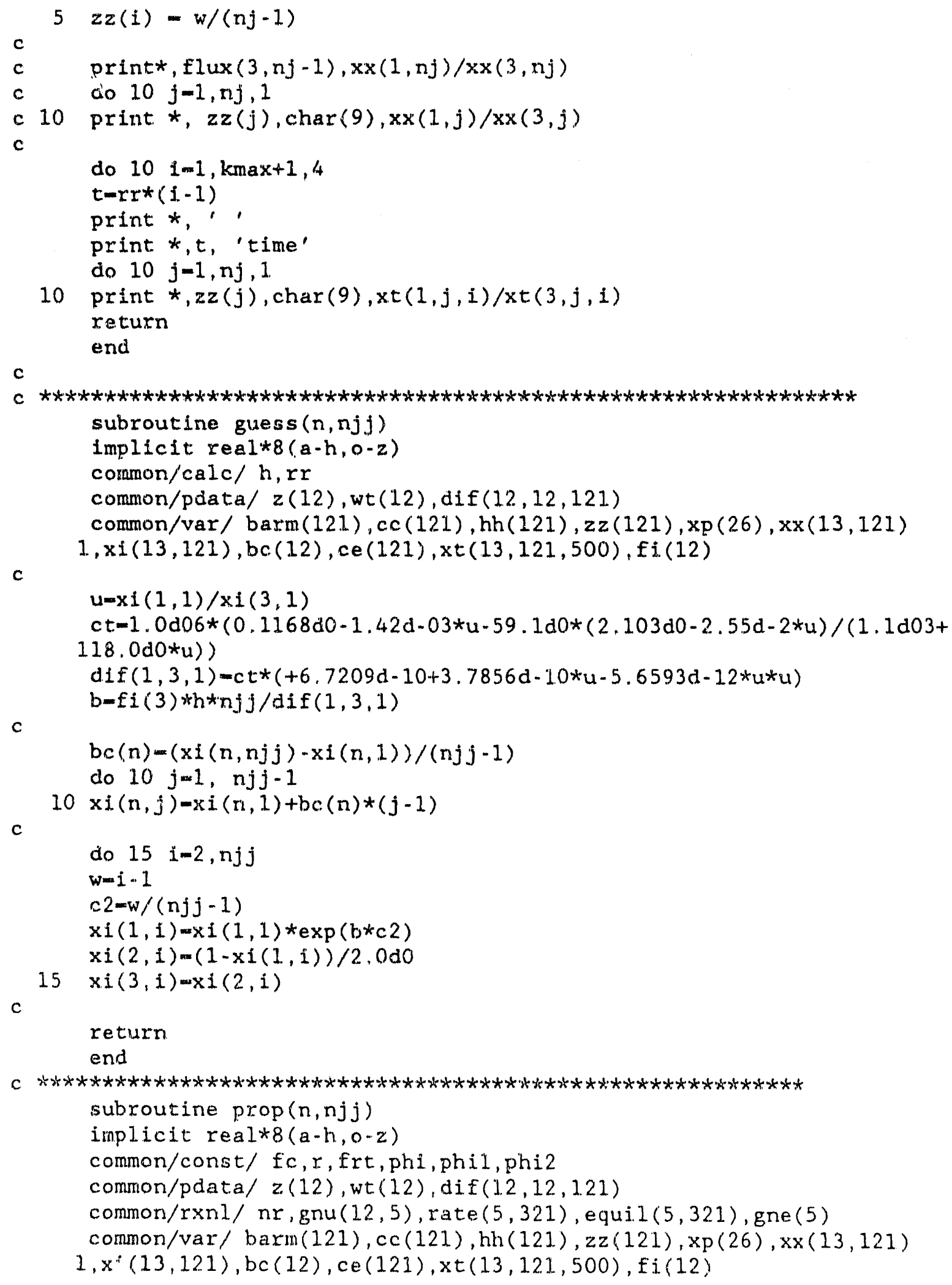


c

$t=353.0 \mathrm{~d} 0$

do $10 j=1, n j j$

$u=x x(1, j) / x x(3, j)$

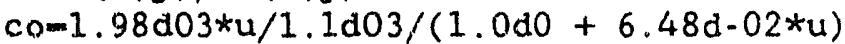

$\mathrm{ch}=1.98 \mathrm{~d} 03 / 1.1 \mathrm{~d} 03 /(1.0 \mathrm{~d} 0+6.48 \mathrm{~d}-02 * \mathrm{u})$

$\mathrm{cm}=1.98 \mathrm{~d} 03 / 1.1 \mathrm{~d} 03 /(1.0 \mathrm{~d} 0+6.48 \mathrm{~d}-02 * \mathrm{u})$

$c c(j)=(3.60 \mathrm{~d} 03+1.8 \mathrm{~d} 03 * \mathrm{u}) /(1.0 \mathrm{~d} 0+6.48 \mathrm{~d} .02 * \mathrm{u})$

ek $=(3.375 d-01 * u-0.2141 d 0) * \operatorname{dexp}(-1.268 d 03 / t)$

$d p=((0.35 d 0 * u) * *(-4.0 d 0)+0.2142 d 0) * *(-0.25 d 0)$

$\mathrm{r} 0=(1.98 \mathrm{~d} 03+32.4 \mathrm{~d} 0 * \mathrm{u}) /(1.0 \mathrm{~d} 0+6.48 \mathrm{~d}-02 * \mathrm{u})$

af $-0.04 \mathrm{~d} 0 * r * t *(((u+61.11 \mathrm{~d} 0) * * 2) / u / u+1.0 \mathrm{~d} 0)$

$\mathrm{df}=\mathrm{r} 0 *(3.5 \mathrm{~d}-06 * \exp (-2.436 \mathrm{~d} 03 / \mathrm{t})) / \mathrm{af}$

$\mathrm{df}=\mathrm{df} * u *(u+61.11 \mathrm{dO}) / 1.10 \mathrm{~d} 0 / 14.0 \mathrm{~d} 0$

$c$

c

calculate Lij's

$a=e k * d p / f c / f c / c h / c o / 1.0 d 04$

$b=\mathrm{df} / \mathrm{co} / \mathrm{co} / 1.0 \mathrm{~d} 06+\mathrm{ek} * \mathrm{dp} * \mathrm{dp} / \mathrm{co} / \mathrm{co} / \mathrm{fc} / \mathrm{fc} / 1.0 \mathrm{~d} 04$

c

$c=\mathrm{ek} / \mathrm{fc} / \mathrm{fc} / \mathrm{ch} / \mathrm{ch} / 1.0 \mathrm{~d} 04$

c

c

c calculate Dij's

calculate Kij's

$x 1-a /(-a * a+b * c)$

$y 1=(c-a) /(-a * a+b * c)$

$z i 1=(b-a) /(-a * a+b * c)$

$\operatorname{dif}(1,3, j)=1.0 \mathrm{~d} 06 * r * t * \cot * \mathrm{ch} / \mathrm{x} 1$

dif $(2,3, j)=1.0 \mathrm{~d} 06 * r * t * \mathrm{ch} * \mathrm{crn} / z 1$

$\operatorname{dif}(1,2, j)-1.0 \mathrm{~d} 06 * r * t * \operatorname{co} * \mathrm{~cm} / \mathrm{yl}$

$\operatorname{dif}(2,1, j)=\operatorname{dif}(1,2, j)$

$\operatorname{dit}(3,1, j)=\operatorname{dif}(1,3, j)$

$10 \operatorname{dif}(3,2, j)=\operatorname{dif}(2,3, j)$

te $=\mathrm{ek} * \mathrm{dp} * \mathrm{dp} * \mathrm{r} * t / \mathrm{co} / \mathrm{df} / \mathrm{fc} / \mathrm{fc} / 10.0 \mathrm{~d} 0$

c

te $-2.0 \mathrm{~d}-02 *(1.0 \mathrm{~d} 0+$ te $) / \mathrm{ek}$.

return

end 


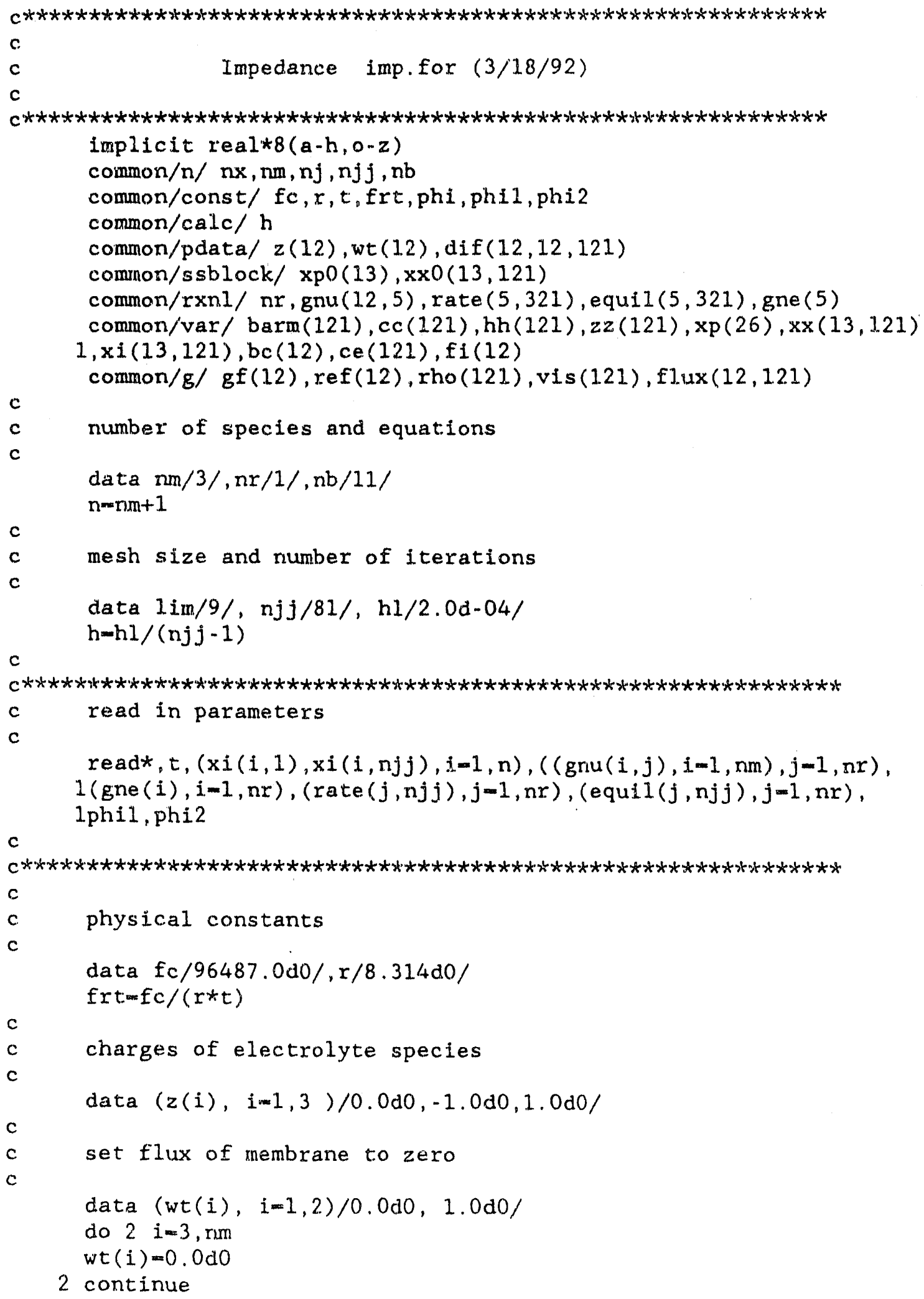




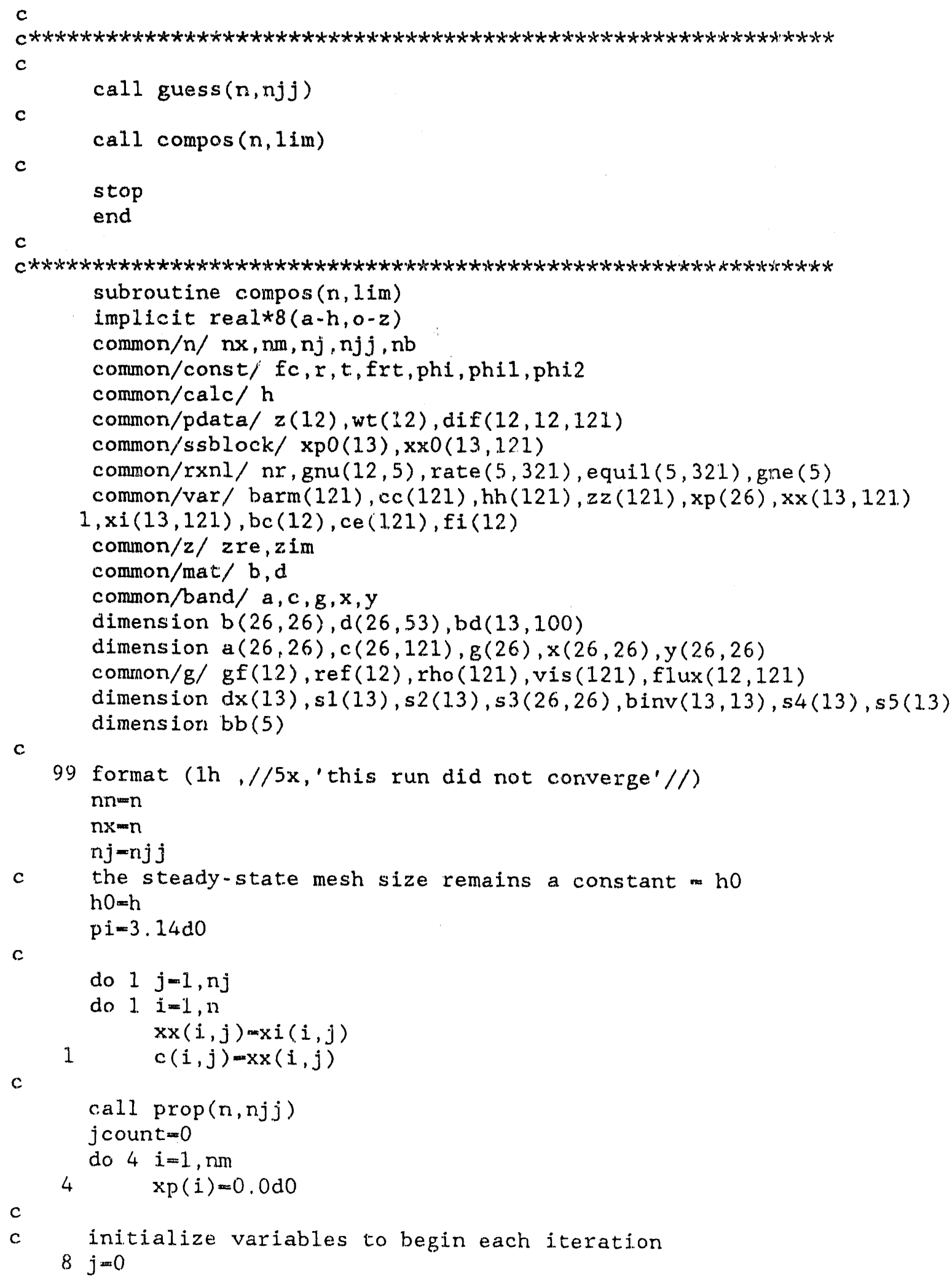




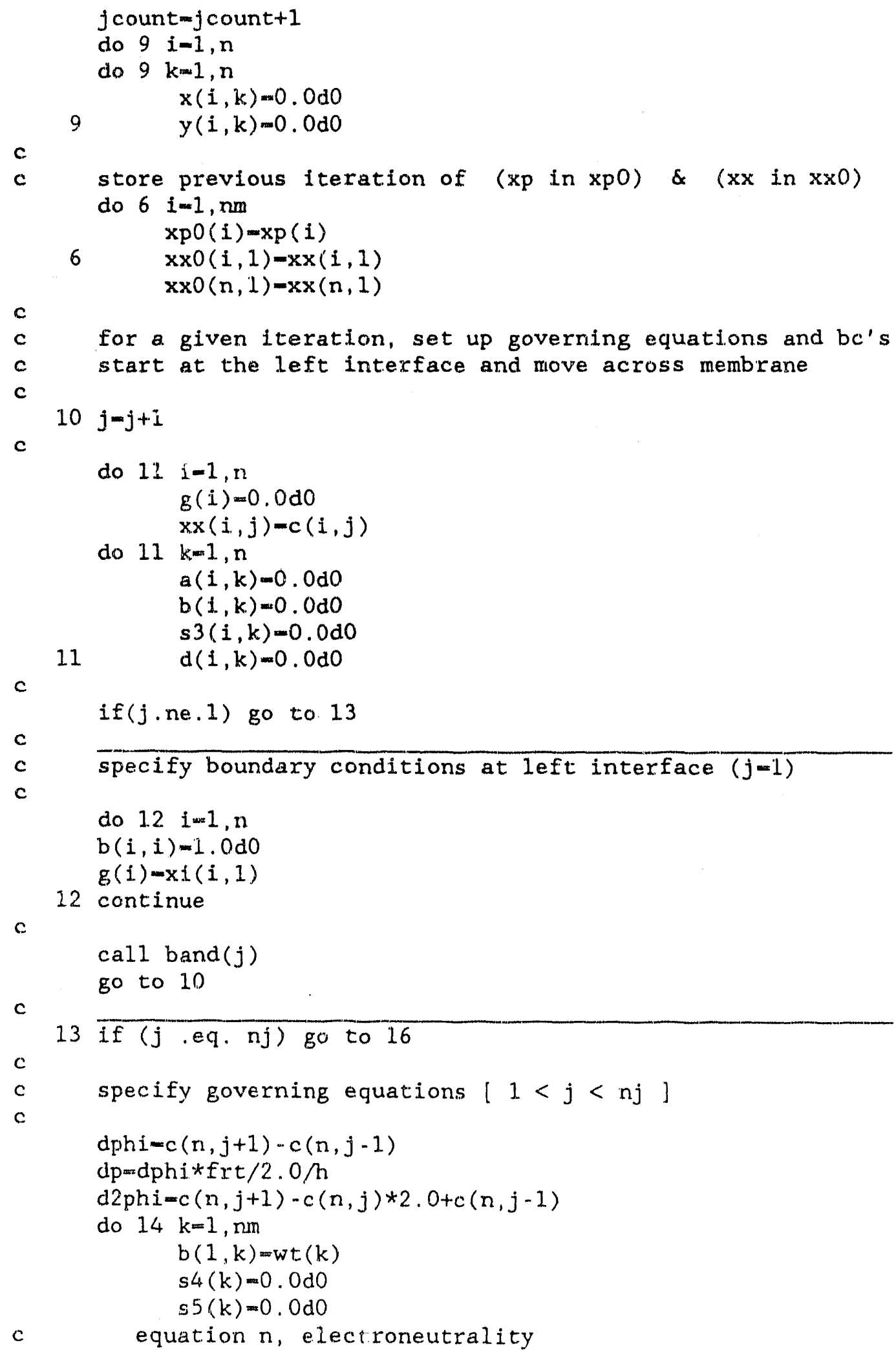


$\mathrm{b}(\mathrm{n}, \mathrm{k})-\mathrm{z}(\mathrm{k})$

$d(k, k)=1.0 d 0$

$d x(k)=c(k, j+1)-c(k, j-1)$

if (k.eq.1) go to 14

do $18 \mathrm{i}=1, \mathrm{~nm}$

if (i.eq.k) go to 18

$b(k, i)=c(k, j) / \operatorname{dif}(i, k, j)$

$b(k, k)=b(k, k)-c(i, j) / \operatorname{dif}(i, k, j)$

14 continue

continue

$d(k, n)=(d x(k)+z(k) * f r t * c(k, j) * d p h i) / 2.0 / h$

call matinv( $\mathrm{nm}, \mathrm{n}$, determ)

do $20 \quad i=1, n m$

$f \operatorname{lux}(i, j)-d(i, n)$

do $19 \mathrm{~km}=1, \mathrm{~nm}$

$\operatorname{binv}(i, k)-d(i, k)$

19

$b(i, k)=0.0 \mathrm{~d} 0$

$b(i, n)=0.0 \mathrm{~d} 0$

$d(i, n)=0.0 d 0$

c

equation 1 , the sum of mole fractions equals 1.0

$20 \mathrm{~b}(1, \dot{1})=1.0 \mathrm{~d} 0$

do $22 \quad i=1, \mathrm{~nm}$

$s i(i)=0.0 \mathrm{~d} 0$

s2(i) $=0.0 \mathrm{do}$

do $22 \mathrm{k}=1, \mathrm{~nm}$

if(k.eq.i) go to 22

a2-flux $(k, j) / \operatorname{dif}(i, k, j)$

$s 4(i) \operatorname{ars} 4(i)+f l u x(k, j) / \operatorname{dif}(i, k, j+1)$

s5 (i) $=\mathrm{s} 5(i)+f l u x(k, j) / \operatorname{dif}(1, k, j-1)$

al $-(c(k, j+1) / \operatorname{dif}(i, k, j+1)-c(k, j-1) / \operatorname{dif}(i, k, j-1)) / 2.0 / h$

$s 1(i)=s 1(i)+a l$

$s 2(i)=s 2(i)+a 2$

21

do $21 \quad 1=1, \mathrm{~nm}$

22 continue

do $15 \quad i=2, \mathrm{~nm}$

$g(i)=g(i)-f l u x(i, j) * s l(i)$

$s 4(i)=s 4(i) / 2.0 \mathrm{dO} / \mathrm{h}$

$\mathrm{s} 5(\mathrm{i})=.\mathrm{s} 5(\mathrm{i}) / 2.0 \mathrm{~d} 0 / \mathrm{h}$

do $27 \mathrm{k}=1, \mathrm{~nm}$

$s 3(k, i)=-s 3(k, i)-b i n v(i, k) * s 1(i)$

if $(k . e q .1)$ go to 5

$d(i, k)=d(i, k)+s 3(k, i)$

$d(i, n)=d(i, n)+z(k) * c(k, j) * f r t * s 3(k, i) / 2.0 / h$

$a(i, n)=a(i, n)-z(k) * c(k, j) * f r t * s 3(k, i) / 2.0 / h$

5

27

continue if (k.eq.i) go to 27

do $24 \mathrm{k}=\mathrm{l}, \mathrm{nm}$

$d(i, k)=d(i, k) / 2.0 \mathrm{~d} 0 / \mathrm{h}$ 


$$
a(i, k)=-d(i, k)
$$

do $231=2$, nm if $(k$.eq. I) go to 24 $g(i)-g(i)+b(i, k) * c(k, j)$

\section{5 continue}

$$
\text { continue }
$$

$$
\mathrm{d}(\mathrm{i}, \mathrm{i})=\mathrm{d}(\mathrm{i}, \mathrm{i})+\mathrm{s} 4(\mathrm{i})-1.0 / \mathrm{h} / \mathrm{h}-\mathrm{z}(\mathrm{i}) * \mathrm{dp} / 2.0 / \mathrm{h}
$$

$$
\begin{aligned}
& a(i, i)=a(i, i)-s 5(i)-1.0 / h / h+z(i) * d p / 2.0 / h \\
& g(i)=g(i)+b(i, i) * c(i, j)+s 4(i) * c(i, j+1)-s 5(i) * c(i, j-1)
\end{aligned}
$$$$
-z(i) * f r t *(c(i, j) * d 2 p h i+0.25 * d x(i) * d p h i) / h / h
$$

$a(i, n)-a(i, n)+z(i) * \operatorname{frt} *(d x(i) / 4.0-c(i, j)) / h / h$

$b(i, n)=z(i) * f r t * 2.0 * c(i, j) / h / h$

$d(i, n)-d(i, n)-z(i) * f r t *(d x(i) / 4.0+c(i, j)) / h / h$

$b(i, i)=b(i, i)+2.0 / h / h-d 2 p h i * z(i): f \operatorname{frt} / h / h$

$c$

$$
\begin{aligned}
& g(1)=1.0 \mathrm{~d} 0 \\
& \text { call band }(j) \\
& \text { go to } 10
\end{aligned}
$$

c

16 continue specify boundary conditions at right interface $(j-n j)$

$c$

phi-phi2

dphiom- $(2.0 \mathrm{~d} 0 * c(n, n j-1)-1.5 \mathrm{~d} 0 * c(n, n j)-0.5 \mathrm{~d} 0 * c(n, n j-2)) / \mathrm{h}$

do $66 \mathrm{i}=2, \mathrm{~nm}$

$a(i, i)=-2.0 \mathrm{~d} 0 / \mathrm{h}$

$y(i, i)-+0.5 \mathrm{~d} 0 / \mathrm{h}$

$b(i, i)-b(i, i)+1.5 \mathrm{~d} 0 / \mathrm{h}$

$\operatorname{coe}=z(i) * c(i, n j) * f r t$

$a(i, n)-a(i, i) * c o e$

$y(i, n)=y(i, i) * c o e$

$b(i, n)=b(i, n)+b(i, i) * c o e$

$b(i, i)=b(i, i)+z(i) * f r t * d p h i o$

c $g(i)=g(i)+c o e * d p h i o$

$b(n, i)-z(i)$

$\mathrm{b}(1, i)=1.0 \mathrm{~d} 0$

$b(1,1)=1.0 \mathrm{~d} 0$

$g(1)=1.0 \mathrm{~d} 0$

$b(n, 1)=z(1)$

$g(n)=0.0 \mathrm{~d} 0$

if (nr.eq.0) go to 38

do $i=1, n r$

$\mathrm{bb}(\mathrm{i})=0.5 \mathrm{do}$

end do 


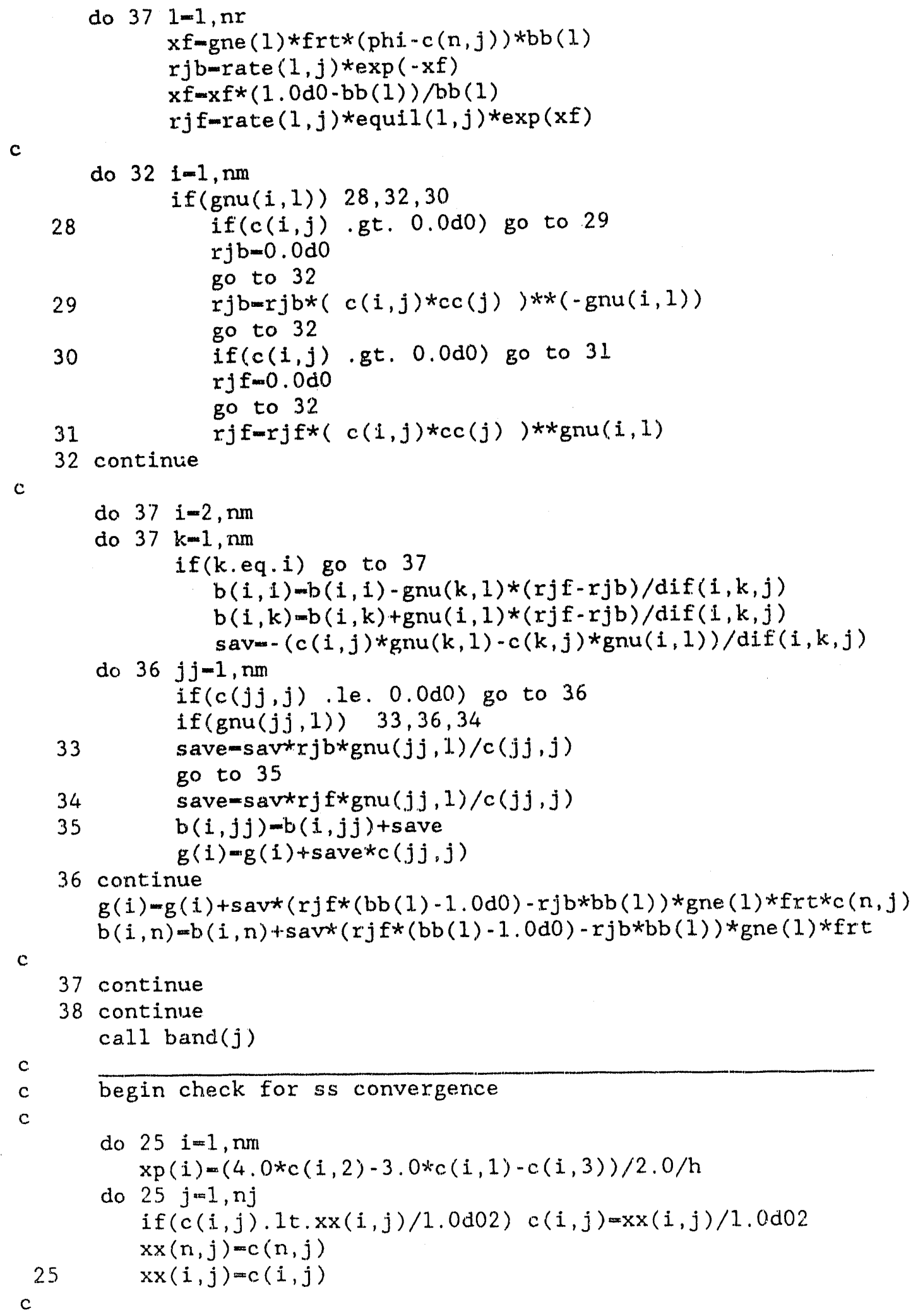




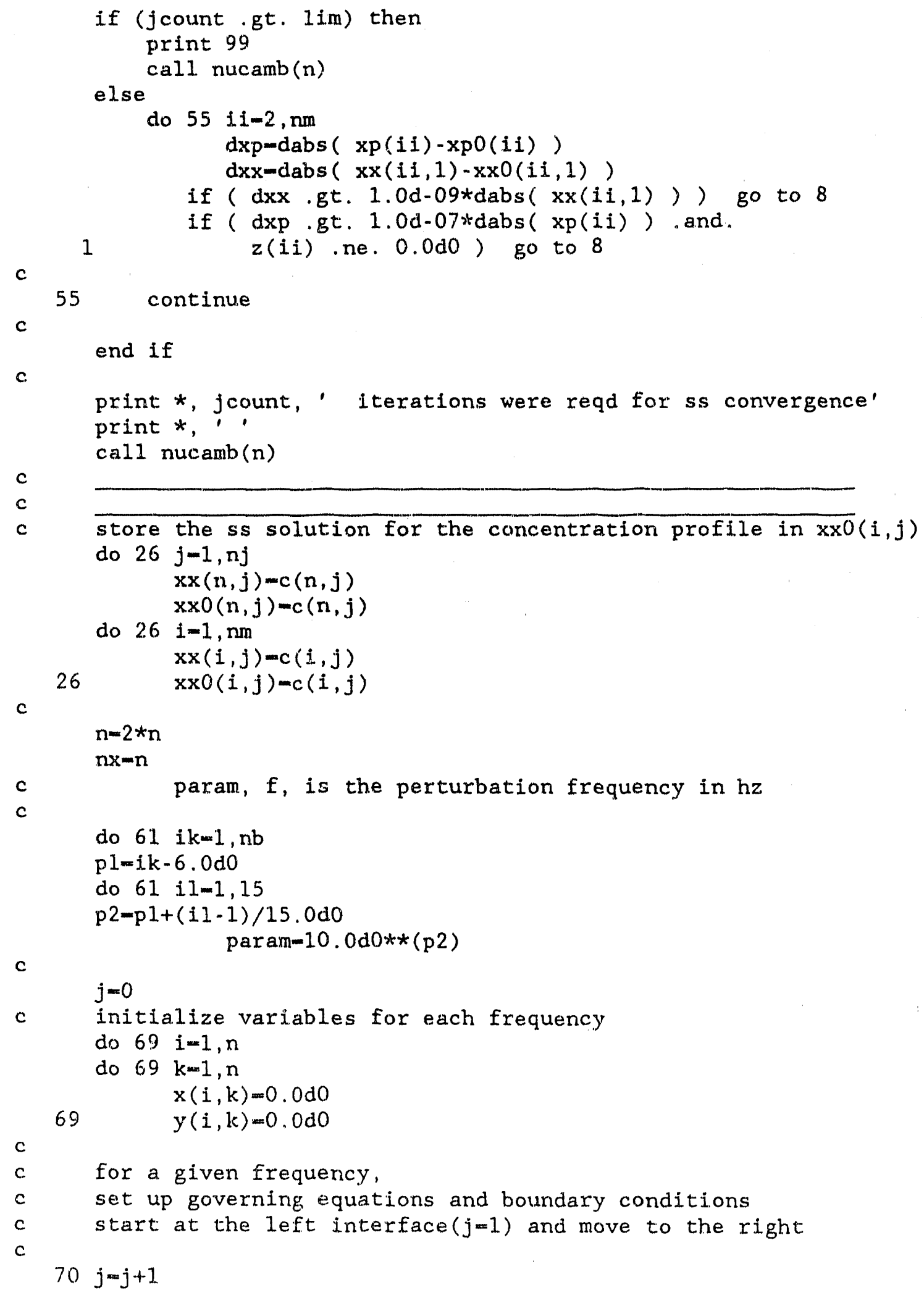


c

do $71 \mathrm{i}=1, \mathrm{n}$

$g(i)=0.0 \mathrm{~d} 0$

do $71 \mathrm{k}=1, \mathrm{n}$

$a(i, k)=0.0 \mathrm{~d} 0$

$b(i, k)=0.0 \mathrm{~d} 0$

s3 $(i, k)=0.0 \mathrm{~d} 0$

71 $\mathrm{d}(1, k)=0.0 \mathrm{~d} 0$

c

if $(j$, ne. 1$)$ go to 73

c.

c

$c$ specify boundary conditions at the left interface $(j-1)$

do $72 i=1, n$

$72 b(1,1)-1.0$ do

c

call band $(j)$

c

go to 70

c

73 if $(j$.eq. nj ) go to 76

$c$

c

$c$

c

specify governing equations $[1<j<n j]$

dphimxx $(n n, j+1)-x x(n n, j-1)$

dp-dphi $* f r t / 2.0 / h$

d2phi=xx $(n n, j+1)-x x(n n, j) * 2.0+x x(n n, j-1)$

do $74 \mathrm{k}-1, \mathrm{~nm}$

$b(1, k)=w t(k)$

$s 4(k)-0.0$

$s 5(k)=0.0$

equation $\mathrm{nn}$, electroneutraity

$\mathrm{b}(\mathrm{nn}, \mathrm{k})=\mathrm{z}(\mathrm{k})$

$d(k, k)=01.0$

$d x(k)=x x(k, j+1)-x x(k, j-1)$

if $(k$ eq. 1$)$ go to 74

do $78 \mathrm{i}=1, \mathrm{~nm}$

if $(1$.eq. $k)$ go to 78

$b(k, i)-x x(k, j) / \operatorname{dif}(i, k, j)$

78

$b(k, k)-b(k, k)-x x(i, j) / d i f(i, k, j)$

74

continue

$d(k, n n)-(d x(k)+z(k) * f r t * x x(k, j) * d p h i) / 2.0 / h$

continue

call matinv ( $\mathrm{nm}, \mathrm{nn}$, determ)

do $80 \quad i=1, n m$

$\operatorname{flux}(i, j)=d(i, n n)$

do $79 \mathrm{k}=1, \mathrm{~nm}$

binv $(i, k)=d(i, k)$

79

$b(i, k)=0.0 \mathrm{~d} 0$

$\mathrm{d}(\mathrm{i}, \mathrm{k})=0.0 \mathrm{~d} 0$ 


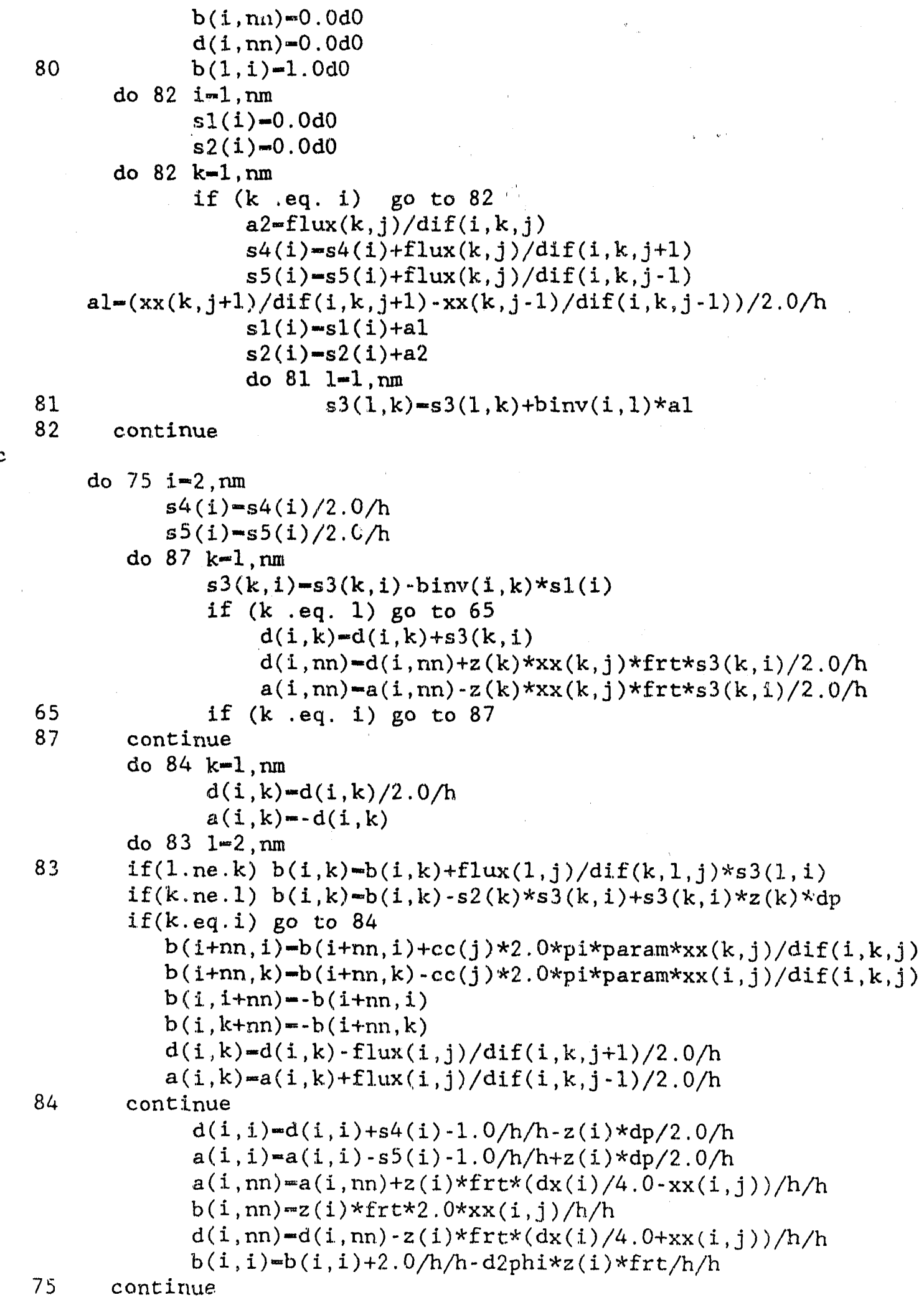


$c$

c governing equations unique to ac problem

do $68 \mathrm{i}-1, \mathrm{nn}$

do $68 \mathrm{k}=1$, nn

$a(1+n n, k+n n)-a(1, k)$

68

$b(1+n n, k+n n)-b(1, k)$

c

call band( $j)$

go to 70

c

76 continue

$c$

specify boundary conditions at right interface(j-nj)

phi-phit2

dphio-(-2.0d0*xx(nn, nj-1)+1.5d0*xx $(n n, n j)+0.5 d 0 * x x(n n, n j-2)) / h$ do $366 \quad 1-2$, num

$a(i, i)=-2.080 / \mathrm{h}$

$y(i, i)=0.5 \mathrm{~d} 0 / \mathrm{h}$

$\mathrm{b}(i, i)=+1.5 \mathrm{~d} 0 / \mathrm{h}$

$\cos -z(i) * 2 x(i, n j) *$ frt

$a(1, n n)-a(1,1) * c o e$

$y(1, n n)-y(1,1) * c o e$

$b(1, n n)-b(1,1) * 60$

$b(1,1)-b(1,1)+z(1) \star f r t * d p h 10$

$b(n \pi, 1)-z(1)$

$3.66 \quad b(1,1)-1.0 \mathrm{~d} 0$

$b(1,1)-1.0 d 0$

c

$b(n n, 1)-z(1)$

If $(\mathrm{nr}$.eq.0) go to 338

do $1-1$, nr

$b b(i)=0.5 d b$

end do

do $3371-1, \mathrm{nr}$

$x f-g n e(1) * f r t *(p h i-x x(n n, j)) * b b(1)$

$r f b=r a t e(1, j) * \exp (-x f)$

$x f=x f *(1.0 d 0-b b(1)) / b b(1)$

rjf-race $(1, j) * \operatorname{equ} f(l, j) * \exp (x f)$

c

do $332 i=1$, nun

if $(\operatorname{grau}(1,1)) \quad 328,332,330$

if $(x \times(1, j), g t .0 .000)$ go to 329

$r j b=0.0 d 0$

go to 332

329

$r j b=r j b *(x \times(i, j) * c c(j)) * *(-\operatorname{gnu}(i, j))$

330 go to 332

if $(x x(i, j), g t .0 .00)$ go to 331

r. $f=0.080$

go to 332 
$c$

$v v=1.0 d-04 *$ frt

do $337 \quad 1=2, \mathrm{~nm}$

do $337 \mathrm{k}=1, \mathrm{~nm}$

if $(k, e q . j)$ go to 337

$b(i, i)-b(i, i)-g n u(k, 1) *(r j f-r j b) / d i f(i, k, j)$

$b(1, k)-b(i, k)+\operatorname{gnu}(i, 1) *(r j f-r j b) / d i f(i, k, j)$

do $336 j j=1, \mathrm{~nm}$

sav- $-(x x(i, j) * \operatorname{gnu}(k, 1)-x x(k, j) * \operatorname{gnu}(i, 1)) / \operatorname{dif}(i, k, j)$

333

if $(c(j j, j)$. Ie. $0.0 \mathrm{do})$ go to 336

if $(\operatorname{gnu}(j j, 1)) \quad 333,336,334$

334 save-sav*rjb*gnu $(j j, 1) / x x(j j, j)$ go to 335 


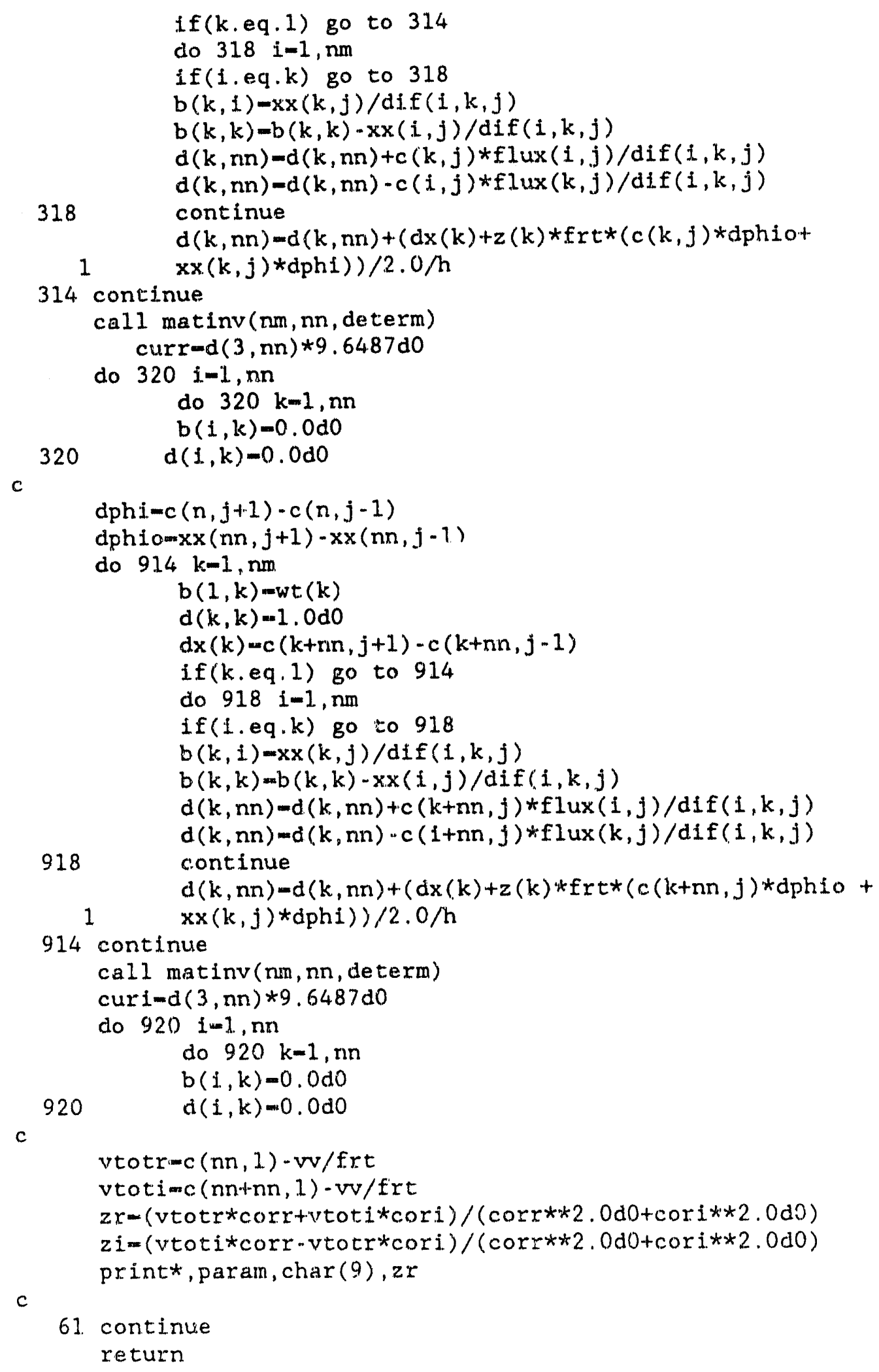


Chapter 8

Convective Diffusion near a Consolute Point

\subsection{Introduction}

The driving force for diffusion is the gradient of chemical potential of the diffusing species. Usually, one defines the diffusion coefficient in terms of a gradient in concentration. Thus, in the vicinity of a consolute point, the temperature and composition where two liquid phases become completely miscible, this diffusion coefficient becomes zero. Krichevski and Tshekhanskaya [1] and Vitagliano et al.[2] observed this experimentally in the watertriethylamine system. Krichevski and Tshekhanskaya also measured the rate of dissolution of a rotating disk of terephthalic acid in the water-triethylamine system. Levich [3] examined this system theoretically, accounting for variations in the diffusion coefficiert with the concentration of triethylamine as well as the effects of variable physical properties on the hydrodynamics. However, Levich made a number of untenable assumptions, which call to question the validity of his conclusions. Although the behavior of transport properties at a consolute point is often discussed in the literature, see Sengers [4] and Cussler[5] for example, convective-diffusion problems when a consolute point is present in the system have not been addressed other than in Levich's original work.

Our objective is to reexamine convective diffusion to a rotating disk theoretically, without arbitrary assumptions, and to elucidate 
better the behavior of the system in the region of the consolute point. In the analysis below we do not consider variations in the density or viscosity. Glearly, variations in physical properties affect the hydrodynamics and convective diffusion to the disk. Nevertheless, these effects are omitted here for two reasons. First, Hsueh and Newman[6], and others[7] [8] have treated variable physical properties previously. Second, variations in the hydrodynamics will have only a secondary effect on convective diffusion near the consolute point and will add little to our understanding of the behavior in this region. The principal effect we wish to investigate is the consequence of a zero diffusion coefficient at a point in the flow and mass-transfer process.

\section{B. 2 Analysis}

The rotating disk is uniformly accessible to mass transfer. The convective diffusion equation in terms of the mass fraction of reacting species in a binary fluid is [9]

$$
\rho v_{z} \frac{d \omega}{d z}=\frac{d}{d z}\left(\rho D \frac{d \omega}{d z}\right)
$$

The general boundary conditions considered are

$$
\begin{aligned}
& \omega=\omega_{0} \text { at } z=0, \\
& \omega=\omega_{\infty} \text { at } z=\infty .
\end{aligned}
$$

At high schmidt number, the diffusion layer is much smaller than the 
hydrodynamic boundary layer, and the velocity normal to the surface of the disk may be accurately written as [3]

$$
v_{z}=-K z^{2}--a \Omega^{3 / 2} \nu^{-1 / 2} z^{2}
$$

With the dimensioniess variable

$$
\xi=\left(\frac{K}{3 D_{0}}\right)^{1 / 3} z
$$

where $\xi$ can be regarded as the axial distance $z$ divided by the thickness of the diffusion layer and $D_{0}$ is the value of the diffusion coefficient at infinite dilution, equation 1 becomes

$$
\xi^{2} \frac{d \omega}{d \xi}=\frac{d}{d \xi}\left(\frac{D}{3 D_{\circ}} \frac{d \omega}{d \xi}\right)
$$

The implicit solution to equation 4 is

$$
\theta=\frac{\omega-\omega_{0}}{\omega_{\infty}-\omega_{0}}=\frac{\int_{0}^{\xi} \frac{D_{0}}{D} \exp \left\{\int_{0}^{\xi}-\frac{3 D_{0}}{D} \xi^{2} d \xi\right\} d \xi}{\int_{0}^{\infty} \frac{D_{0}}{D} \exp \left\{\int_{0}^{\infty}-\frac{3 D_{0}}{D} \xi^{2} d \xi\right\} d \xi} .
$$

For constant diffusion coefficients, Levich gave the solution

$$
\theta=\frac{1}{\Gamma(4 / 3)} \int_{0}^{\xi} e^{-x^{3}} d x
$$


When the diffusion coefficient is a function of concentration, equation 5 can be integrated numerically, iteration being necessary because $D$ depends on $\omega$. For a solution with a consolute point, however, the diffusion coefficient becomes zero at some value of $\xi$, and equation 5 cannot be integrated directly.

The diffusion coefficient based on a concentration driving force can be related to a diffusion coefficient based on a chemicalpotential driving force through an activity correction

$$
D=D_{0}\left\{1+\frac{d \ln \gamma_{1}}{d \ln x_{1}}\right\}
$$

If the Gibbs energy is expressed by a three-suffix Margules equation, then the activity coefficient for a two-component solution is given by

$$
\ln \gamma_{1}=\frac{A_{12}^{*}}{R T} \mathrm{x}_{2}^{2}+\frac{B_{112}^{*}}{R T} \mathrm{x}_{2}^{2}\left(4 \mathrm{x}_{1}-1\right)
$$

At the consolute point

$$
\frac{\partial^{2} \tilde{G}}{\partial x_{1}^{2}}-\frac{\partial^{3} \bar{G}}{\partial x_{1}^{3}}=0
$$

If the concentration at the consolute point is known, these relationships allow the evaluation of the two constants in equation 8 . Reference [1] gives the consolute point for the water-triethylamine system as $17^{\circ} \mathrm{C}$ and 0.261 mass fraction of triethylamine. These 
values are not in exact agreement with others reported in the literature[10] but suffice to illustrate our techrique. Figure 1 shows the activity factor for this system based on the 3-suffix Margules equation and the molar masses of the components. It is evident that the diffusion coefficient is zero at the consolute point. Additionally, one sees that in the vicinity of the consolute point the diffusion coefficient may be approximated by

$$
D=D_{c}\left(\omega-\omega_{c}\right)^{2}
$$

Similar behavior is observed in other systems. [11] The exponent of 2 in equation 9 corresponds to a critical exponent of $\delta=3$ for the classical analysis of the critical isotherm. Nonclassical analysis and experimental data yield a value of about 4.8 for the critical exponent [12]. In appendix $F$, the analysis that follows is repeated for an exponent of 3.8 in equation 9.

In order to solve the convective diffusion equation in the vicinity of the consolute point, we should formulate the problem so that the singularity is removed. The flux near the consolute point is

$$
\frac{n}{\rho}=-D \frac{d \omega}{d z}+v_{c} \omega_{c}
$$

Variations in the velocity and flux are small compared to variations in the diffusion coefficient and concentration in this region; and, assuming that the diffusion coefficient is of the form given in equa- 


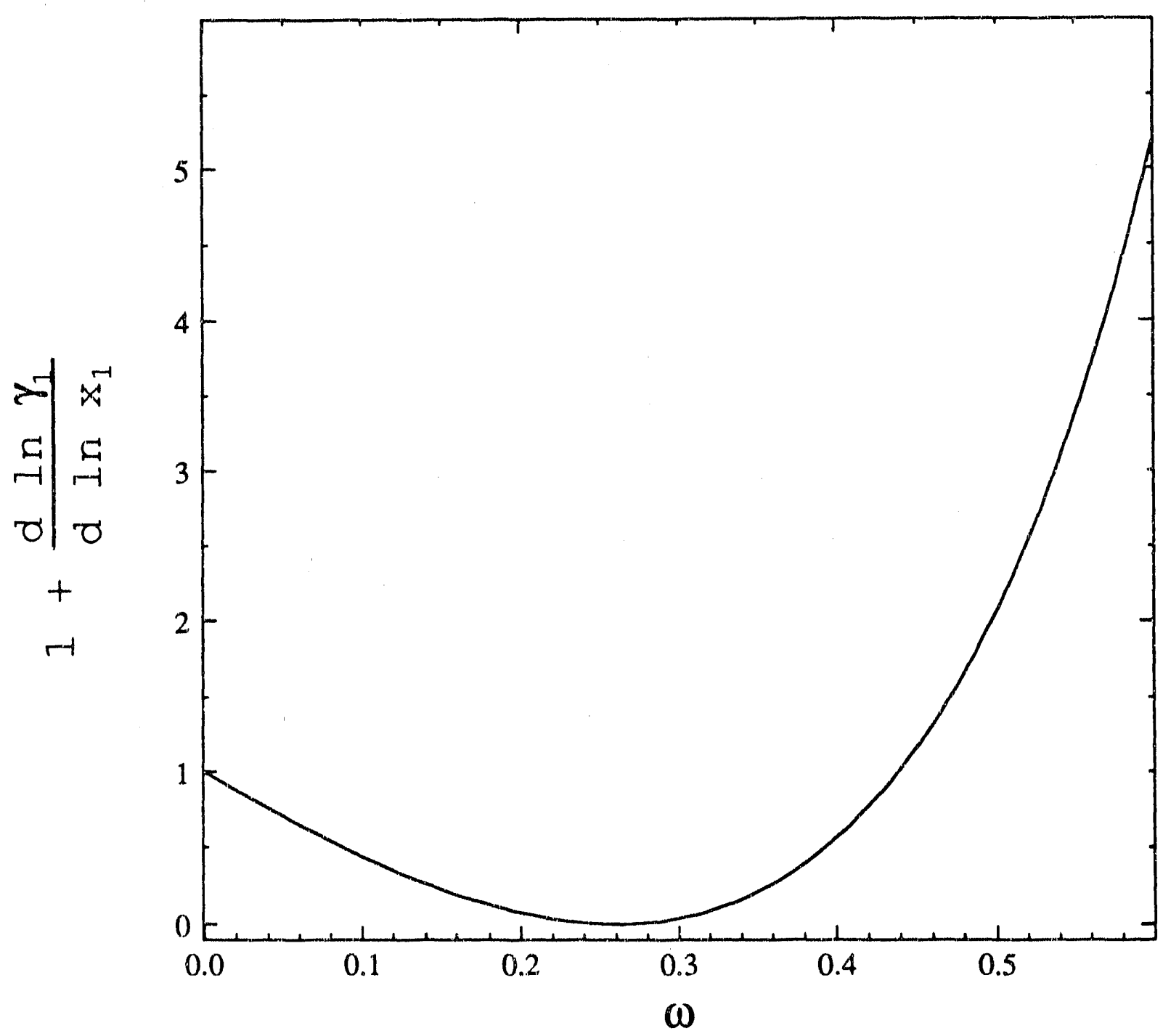

Figure 1. Activity-factor correction to the diffusion coefficient based on the 3-suffix Margules equation. 
tion 9, we conclude that in the vicinity of the consolute point

$$
z-z_{c}-\frac{D_{c}}{3}\left(\omega-\omega_{c}\right)^{3}
$$

where $z_{c}$ is the distance from the surface of the disk to the consolute point, also called the critical distance. Therefore the $z$ coordinate is stretched so that $\omega$ is proportional to $X$ near the consolute point:

$$
x^{3}-z-z c
$$

and equation 1 becomes

$$
v_{z} \frac{d \omega}{d X}=\frac{d}{d X}\left(\frac{D}{3 X^{2}} \frac{d \omega}{d X}\right)
$$

Equation 12 was solved by breaking the problem into two regions: 1) from the consolute point to the surface of the disk, and 2) from the consolute point out to infinity. At the consolute point the concentration is known, but the position is not. The boundary conditions considered were

$$
\begin{gathered}
\omega=\omega_{c} \text { and } \frac{d \omega}{d X}=A \text { at } X=0, \\
\omega=0 \text { at } X=-X_{c} .
\end{gathered}
$$

The concentration at the surface was set equal to zero, representing the reaction of diffusing species at the surface of the disk with 
fast kinetics. For a given value of $X_{c}$, the slope $A$ at the critical point was adjusted to give a zero concentration at the surface. The bulk concentration was then determined by using the same value for the slope at the consolute point and integrating out to infinity. [Equation 12 also was put in a form like equation 5 and solved by numerical integration, but this did not prove to be an efficient method.]

The problem was reformulated with the following variables

$$
x=\frac{-X}{z_{c}^{1 / 3}},
$$

and

$$
W=1-\frac{\omega}{\omega_{c}}
$$

Thus equation 12 becomes

$$
\epsilon\left(1-x^{3}\right)^{2} \frac{d W}{d x}=\frac{d}{d x}\left(\frac{\frac{D}{D_{C}}}{\omega_{c}^{2} x^{2}} \frac{d W}{d x}\right)
$$

where $\epsilon$ is defined by

$$
\epsilon=\frac{3 K z_{c}^{3}}{\omega_{c}^{2} D_{c}} .
$$

This can be split into two first-order differential equations 


$$
\frac{d P}{d x}-\epsilon\left(1-x^{3}\right)^{2} \frac{\omega_{c}^{2} x^{2}}{\frac{D}{D_{c}}} P
$$

$$
\frac{d W}{d x}-\frac{\omega_{c}^{2} x^{2}}{\frac{D}{D_{c}}} P
$$

and solved as an initial value problem with the boundary conditions

$$
W=0, \quad P=P_{0} \text { at } x-0
$$

Equations 16 and 17 were solved using a Runge-Kutta routine. The value of $\epsilon$ was fixed, corresponding to a given critical distance. At $x=0$ the value of $P_{0}$ was adjusted until the calculated surface concentration was zero. Then with the known value of $P_{O^{\prime}}$, the equations were integrated from $x=0$ to $x=-\infty$ to determine the value for the concentration far from the disk. The Runge-Kutta routine gave identical results to the previous method but was considerably more efficient.

Assuming equation 9 is valid near the consolute point does not restrict the validity of the method. It is important that the diffusion coefficient vary as shown in equation 9 only in the vicinity of the consolute point. In the analysis below we assume that the functional, form of equation 9 is valid over all compositions only to illustrate more clearly our method without the introduction of detailed physical-property variations, which would restrict our results to only one physical or chemical system. The concentration profiles for $\omega_{\infty}>\omega_{c}$ are shown in figure 2. Figure 3 shows the con- 


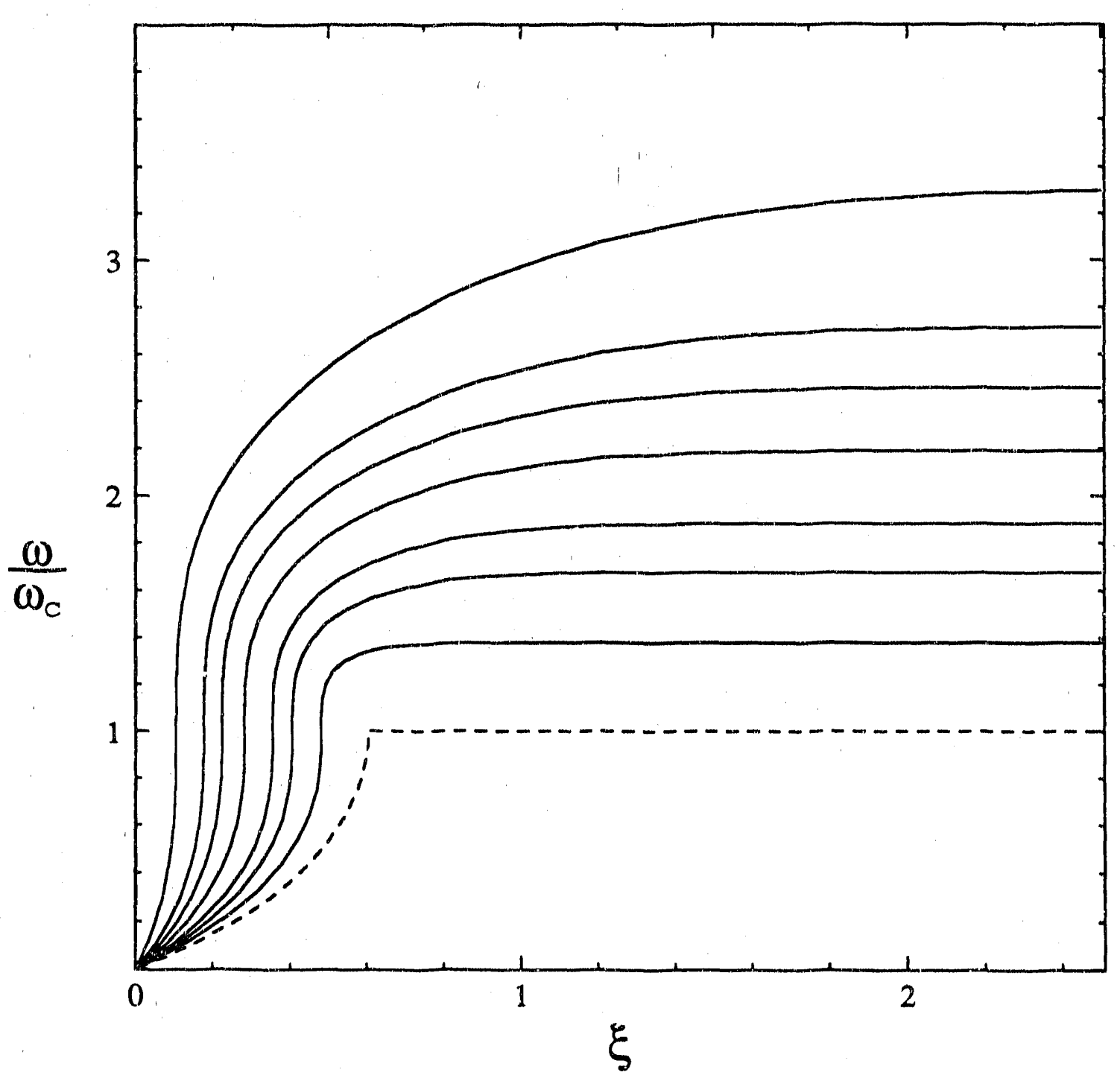

Figure 2. Concentration profiles for bulk concentrations above or at (dashed line) the consolute-point composition. 


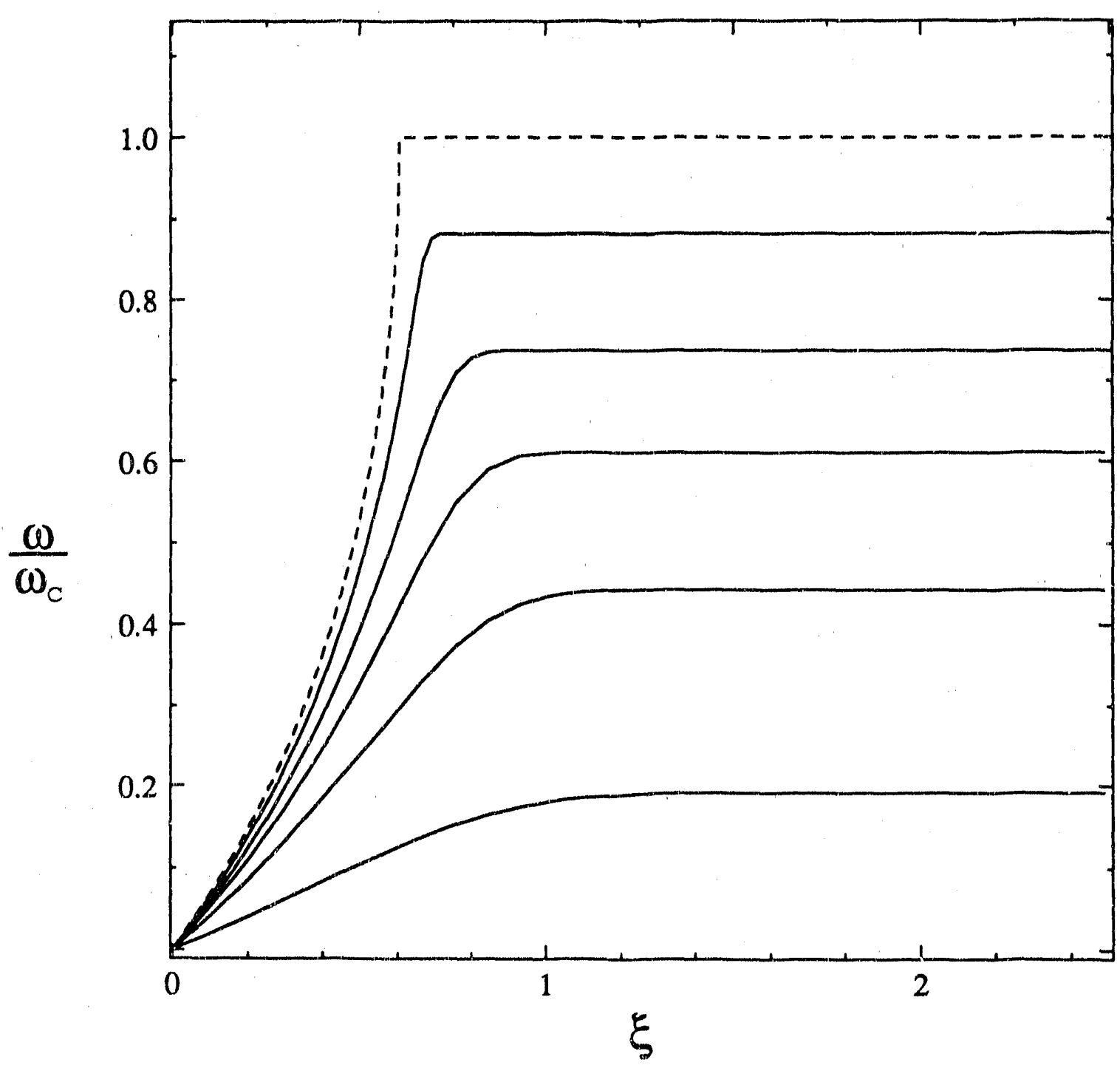

Figure 3. Concentration profiles for bulk concentrations below or at (dashed line) the consolute-point composition. 
centration profiles when the bulk concentration is below the consolute-point composition. In both cases the concentration is plotted against $\xi$, which is related to $x$ by

$$
\xi-\left(1-x^{3}\right)\left(\frac{\epsilon}{9}\right)^{1 / 3}
$$

As the bulk concentration is raised, the consolute point moves closer to the surface of the disk, and the slope $d W / d x$ at this critical distance increases. Figure 4 shows the slope $d W / d x$ at the conso. Iute point as a function of the concentration far from the disk. For values of $\epsilon>2$, solutions were not possible. We wished to investigate the behavior when the bulk concentration was arbitrarily close to that of the consolute composition, and to determine the maximum value of $\epsilon$.

\subsection{Perturbation Analys 1 s}

Figure 5 shows $W$ against $x$, which is related to the distance From the critical point by equation $1 B$. The stretching of the above formulation eliminated any sharp variation in this curve, in contrast to figure 2. As $\omega_{\infty}$ is lowered toward the consolute-point composin tion, $\epsilon$ iniseases, and $d w / d x$ at $x m 0$ appears to be approaching zero. For $\epsilon=1$ \& the curve shows significant curvature. Suppose there is a value of a for which $d w / d x-0$ at $x=0$. The solution in the outer region would then be $W$ for 0 for $x<0$. For $x>0$ look for a solution of the form 


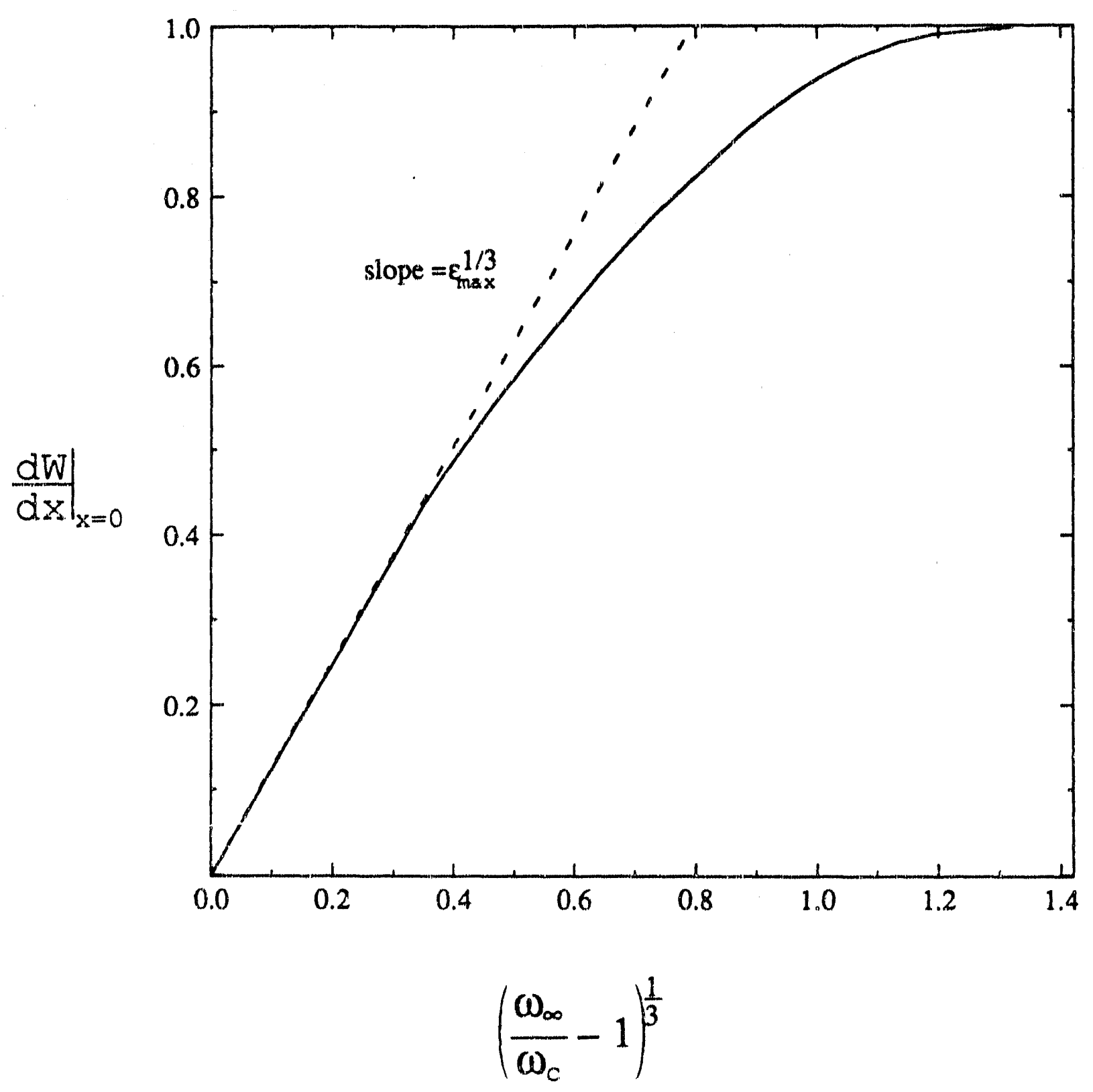

Figure 4. The slope at the consolute point. Dashed line is the asymptote given in equation 28. 


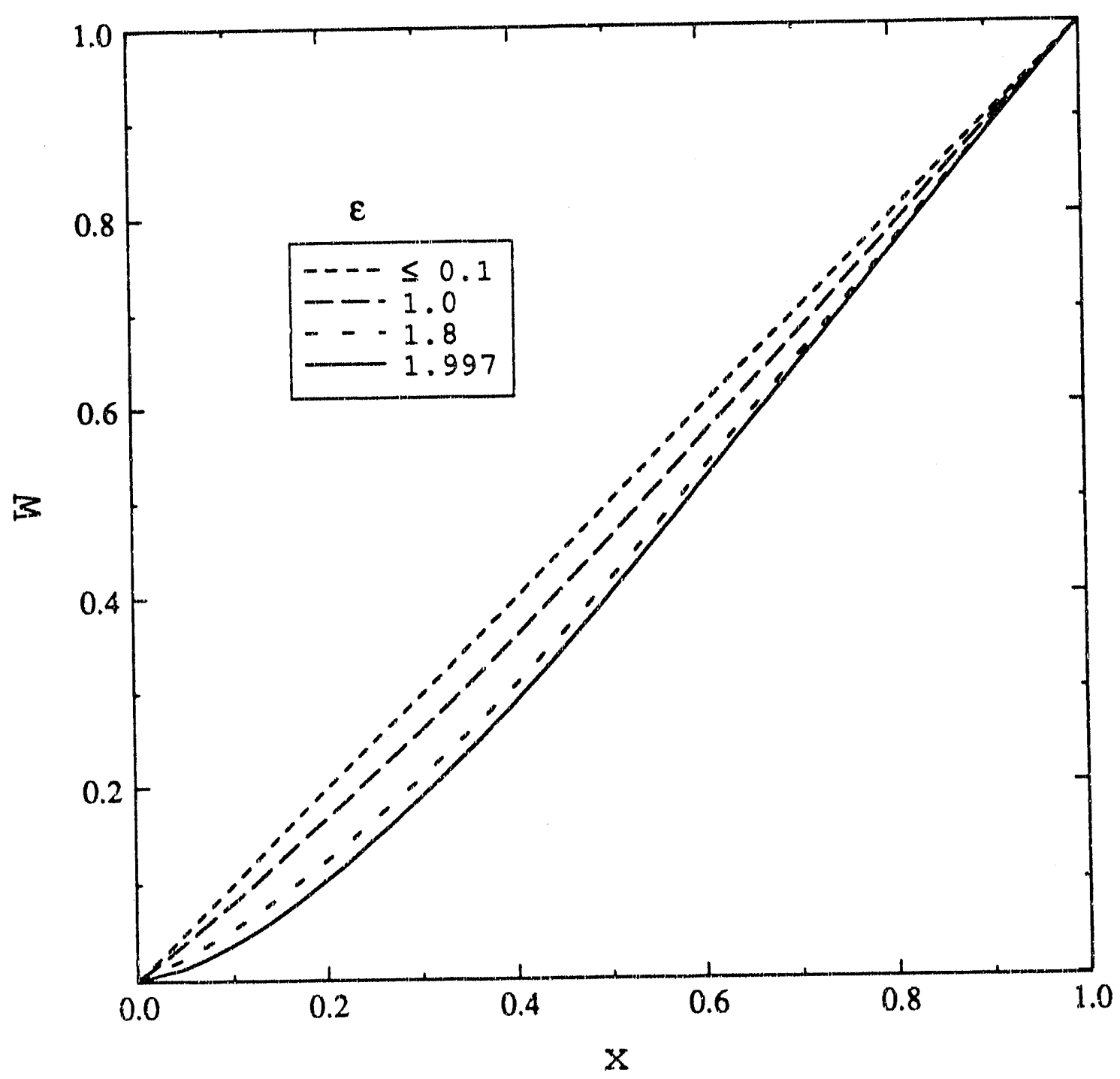

Figure 5. Concentration profiles as the bulk concentration approaches the consolute-point composition. Solid line is for the case when the bulk concentration nearly equals the consolute-point composition. 


$$
W=A x+B x^{2}+\ldots
$$

near $x=0$. Substituting into the differential equation and equating equal powers of $\mathrm{x}$ gives

$$
4 A B=\epsilon
$$

If $\varepsilon$ approaches a limit, somewhat greater that 1.8 , then $B$ approaches infinity as $A$ goes to zero. The region where

$$
A x \approx B x^{2}
$$

defines an inner region (in the sense of a singular-perturbation expansion), Let.

$$
\begin{aligned}
& \bar{x}=\frac{x \epsilon}{4 A^{2}}, \\
& \bar{W}=\frac{\epsilon}{4 A^{3}} W .
\end{aligned}
$$

The problem in the inner region becomes

$$
\left(1-\left(\frac{4 A^{2} \bar{x}}{\epsilon}\right)^{3}\right]^{2} 4 \frac{d \bar{W}}{d \bar{x}}=\frac{d}{d \bar{x}}\left(\frac{\bar{W}^{2}}{\bar{x}^{2}} \frac{d \bar{W}}{d \bar{x}}\right]
$$

with the boundary conditions

$$
\bar{W}=0 \text { and } \frac{d \bar{W}}{d \bar{x}}=1 \text { at } x=0 \text {, }
$$

To a zeroth approximation, $\bar{W}$ satisfies 


$$
4 \frac{d \bar{W}}{d \bar{x}}=\frac{d}{d \bar{x}}\left(\frac{\bar{W}^{2}}{\bar{x}^{2}} \frac{d \bar{W}}{d \bar{x}}\right)
$$

with the implicit solution

$$
\frac{16 \bar{x}^{3}}{3}=2 \bar{W}^{2}-\bar{W}+\frac{1}{4} \ln (1+4 \bar{W})
$$

As $\bar{x} \rightarrow \infty$

$$
\bar{W} \rightarrow\left(\frac{8}{3}\right)^{1 / 2} \bar{x}^{3 / 2}
$$

or

$$
W \rightarrow\left(\frac{2 \epsilon}{3}\right)^{1 / 2} x^{3 / 2}
$$

The outer region satisfies equation 15 . Let $\tilde{x}=x^{3 / 2}$, and the problem becomes

$$
\frac{2 \epsilon}{3}\left(1-\tilde{x}^{2}\right)^{2} \frac{d W}{d \tilde{x}}=\frac{d}{d \tilde{x}}\left(\frac{W^{2}}{\tilde{\mathbf{x}}} \cdot \frac{d W}{d \tilde{x}}\right)
$$

The solution must match the inner solution as $\bar{x} \rightarrow 0$,

$$
W \rightarrow\left(\frac{2 \epsilon}{3}\right)^{1 / 2} \tilde{x} \quad \text { as } \quad \ddot{x} \rightarrow 0
$$

and

$$
W=1 \text { at } \tilde{x}=1 \text {. }
$$

Thus $t$ can be adjusted to give $W=1$ at $\bar{x}=1$. The numerical solution 
gives $\epsilon-1.9967$. The concentration profiles for this condition are the dashed lines in figures 2 and 3 and the solid line in figure 5. We are also able to find an asymptote for $\left.\frac{d W}{d x}\right|_{x=0}$. As $\bar{x} \rightarrow-\infty$

$$
\overline{\mathrm{w}} \rightarrow-\frac{1}{4}-\frac{1}{4} \exp \left(\frac{64 \bar{x}^{3}}{3}\right)
$$

and since $A=\left.\frac{d W}{d x}\right|_{x=0}$

$$
\left.\frac{d W}{d x}\right|_{x=0} \rightarrow\left(\epsilon\left(\frac{\omega_{\infty}}{\omega_{c}}-1\right)\right)^{1 / 3}
$$

This is the dashed line on figure 4.

\subsection{Mass Transfer}

The rate of mass transfer to the surface of the disk is given by

$$
\frac{n}{\rho}=-\left.D \frac{d \omega}{d z}\right|_{z=0}
$$

The coordinate transformation allows us to define a dimensionless rate of mass transfer $j$ and to express it as

$$
j=\left.\frac{1}{\omega_{C}}\left(\frac{3 D_{O}}{K}\right)^{\frac{1}{3}} \frac{d \omega}{d z}\right|_{z=0}=\left.\frac{1}{(3 \epsilon)^{1 / 3}} \frac{d W}{d x}\right|_{x=1}
$$

For a constant coefficient of diffusion, equation 6 shows that $j$ takes the limiting form 


$$
j=\left.\frac{1}{\omega_{c}}\left(\frac{3 D_{0}}{K}\right)^{1 / 3} \frac{d \omega}{d z}\right|_{z=0}=\frac{1}{\Gamma(4 / 3)} \frac{\omega_{\infty}}{\omega_{c}^{1}} .
$$

Figure 6 shows the flux to the surface of the disk from our numerical. calculation as compared with the theoretical line predicted by equation 31 . The results are summarized below in table 1.

\subsection{Discussion}

From figure 2 one observes that the consolute point moves farther from the surface of the disk as the bulk concentration is decreased and reaches a maximum distance $\xi=0.6054$ when the bulk concentration equals the consolute composition. The slope at the conso. lute point is infinite when plotted against $\xi$. Figure 3 shows the behavior as a consolute point is approached from concentrations below the critical value. As the concentration becomes closer to $\omega_{c}$, the slope increases sharply and is infinite when the consolute point is

Table 1 .

\begin{tabular}{|l|l|l|l|c|}
\hline \multicolumn{1}{|c|}{$\epsilon$} & $d W /\left.d x\right|_{X=0}$ & $d W /\left.d x\right|_{X=1}$ & $\frac{\omega_{\infty}}{\omega_{c}}$ & $j$ \\
\hline 0.01 & 0.99809 & 1.00064 & 3.30252 & 3.22036 \\
0.05 & 0.99039 & 1.00321 & 2.71153 & 1.88811 \\
0.1 & 0.98065 & 1.00647 & 2.45832 & 1.50347 \\
0.2 & 0.96069 & 1.01299 & 2.18820 & 1.20103 \\
0.3 & 0.94009 & 1.01961 & 2.01483 & 1.05606 \\
0.4 & 0.91878 & 1.02632 & 1.88162 & 0.96580 \\
0.5 & 0.89668 & 1.03310 & 1.77097 & 0.90250 \\
0.6 & 0.87372 & 1.04000 & 1.67523 & 0.85495 \\
1.0 & 0.77077 & 1.06851 & 1.38024 & 0.74086 \\
1.8 & 0.43457 & 1.13238 & 1.04281 & 0.64545 \\
1.9967 & 0.0 & 1.15076 & 1.0 & 0.63363 \\
\hline
\end{tabular}




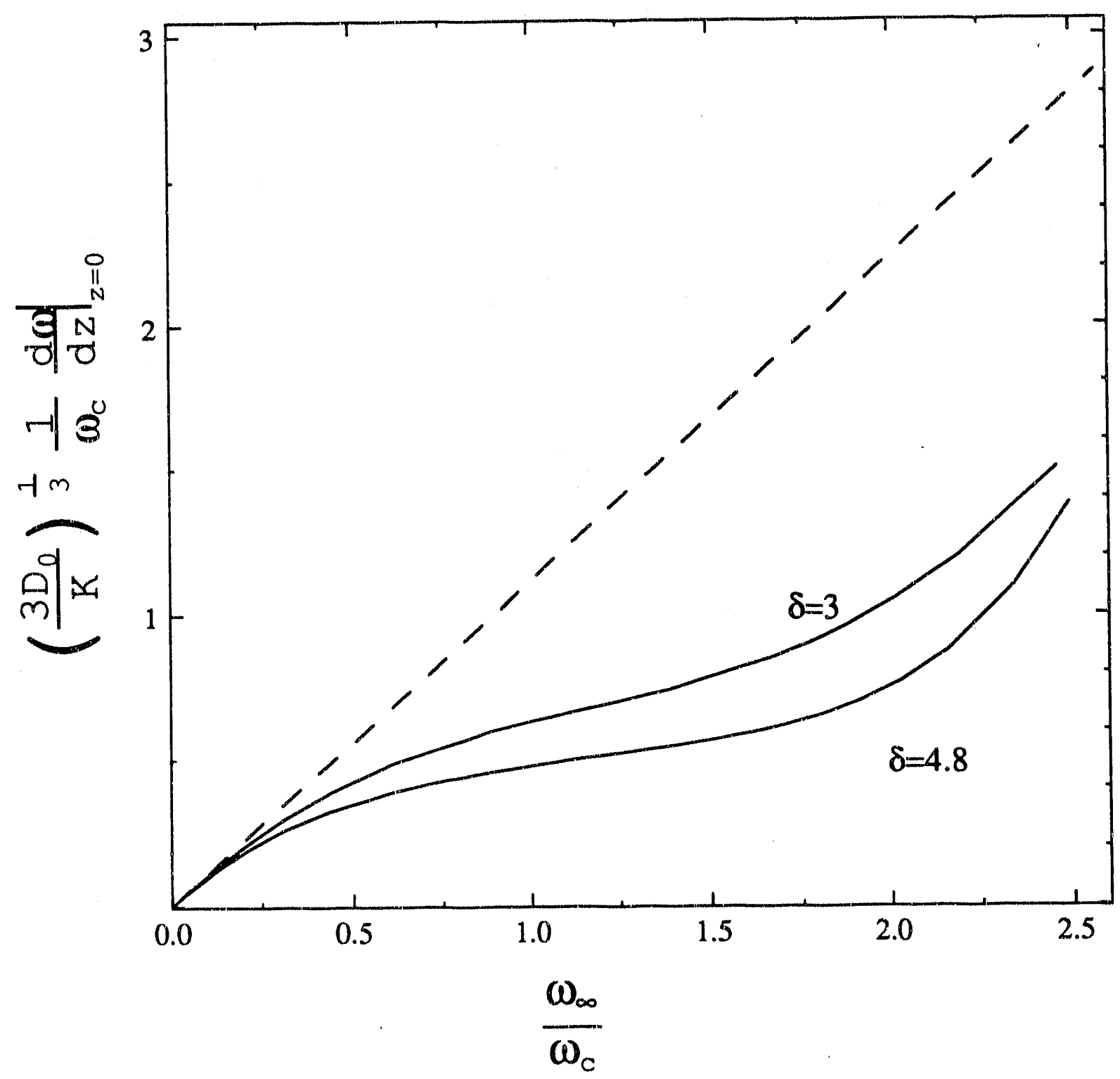

Figure 6. Rate of mass transfer to the surface of a rotating disk. Dashed Iine is that predicted by equation 31 for a constant diffusion coefficient. $\delta=4.8$ is based on the development in Appendix $F$ for nonclassical behavior at the consolute point. 
reached.

At low values of $\omega_{\infty}$, the diffusion coefficient is nearly constant, and the profile approaches that predicted by equation 6 . The rate of mass transfer is therefore identical to that predicted by equation 31 , the dashed line in figure 6 .

As the consolute point is approached, the diffusion coefficient becomes smaller, and the rate of mass transfer is reduced as seen by the leveling off of the solid lines in figure 6 . This does not continue indefinitely because there is a competing effect. As the bulk concentration is increased above the consolute-point composition, the diffusion coefficient increases sharply, and the critical distance moves toward the disk. Thus, the rate of mass transfer becomes larger than that predicted by equation 31 . The leveling off was observed experimentally by Krichevski and Tshekhanskaya [1] and predicted by Levich [3]. The concentration range in Krichevski and Tshekhanskaya's experimer. s was not large enough to verify the upturn in the rate of mass transfer.

Levich arbitrarily divided the problem into three regions: 1) far from the disk the concentration was constant and equal to the bulk concentration, 2) close to the disk the concentration varied approximately linearly with distance from zero to the consolute composition, and 3) a thin intermediate region showed negligible resistance to mass transfer. Figures 2 and 3 clearly contradict this pic. ture. 


\section{List of Symbols}

\begin{tabular}{|c|c|}
\hline$a$ & constant $=0.51023$ \\
\hline$A$ & $\frac{d W}{d x}$ at $x=0$ \\
\hline$A_{12}^{*}$ & Margules constant \\
\hline B & constant in equation 19 \\
\hline$B_{112}^{*}$ & Margules constant \\
\hline$D$ & diffusion coefficient, $\mathrm{cm}^{2} / \mathrm{s}$ \\
\hline$D_{c}$ & parameter used in equation 9 \\
\hline$\tilde{G}$ & Gibbs energy, $\mathrm{J} / \mathrm{mol}$ \\
\hline i & dimensionless flux defined in equation 30 \\
\hline$K$ & parameter in equation 2 \\
\hline$n$ & $\operatorname{mass} \mathrm{flux}, \mathrm{g} / \mathrm{cm}^{2} \cdot \mathrm{s}$ \\
\hline$v_{z}$ & velocity normal to disk, $\mathrm{cm} / \mathrm{s}$ \\
\hline W & dimensionless concentration \\
\hline$x$ & distorted dimensionless distance variable \\
\hline$x_{i}$ & mole fraction \\
\hline$X$ & variable defined below equation $11, \mathrm{~cm}^{1 / 3}$ \\
\hline$z$ & distance from surface of disk, $\mathrm{cm}$ \\
\hline$\gamma_{i}$ & activity coefficient \\
\hline$\Gamma$ & gamma function \\
\hline$\delta$ & critical exponent \\
\hline$\epsilon$ & parameter defined in equation 15 \\
\hline$\theta$ & dimensionless concentration defined by equation 5 \\
\hline$\nu$ & kinematic viscosity, $\mathrm{cm}^{2} / \mathrm{s}$ \\
\hline
\end{tabular}




$\begin{array}{ll}\xi & \text { dimensionless distance } \\ \omega & \text { density, } \mathrm{g} / \mathrm{cm}^{3} \\ \Omega & \text { mass fraction } \\ & \text { rotation speed, rad/s } \\ \infty & \text { fubscripts } \\ \circ & \text { surface of disk, infinite dilution } \\ c & \text { critical or consolute value } \\ - & \text { inner region variable } \\ & \text { outer region variable }\end{array}$

\section{References}

[1] I. R. Krichevsky and Y. V. Tshekhanskaya, "Diffuziya i Rastvoreniya $v$ Zhudkik Rastvorak v Kriticheskoy Oblasty," Zhur. Fiz. Khim. , 30, 2315-2326 (1956).

[2] V. Vitagliano, R. Sartorio, E. Chiaravalle, and O. Ortona, "Diffusion and Viscosity in Water-Triethylamine Mixtures at 19 and $20^{\circ} \mathrm{C}, " \mathrm{~J}$. Chem. Eng. Data, 25, 121-124 (1980).

[3] V. G. Levich, Physicochemical Hydrodynamics, Prentice Hall Inc, Englewood Cliffs, N.J. (1962).

[4] J. V. Sengers, "Transport Processes near the Critical Point of Gases and Binary Liquids in the Hydrodynamic Regime," Ber. 
Busenges. Phys, Chem., 76, 234-249 (1972).

[5] E. L. Cussler, Multicomponent Diffusion, Elsevier Scientific Publishing Company, New York (1976).

[6] L. Hsueh and J. Newman, "The Effect of Variable Transport Properties on Mass Transfer to a Rotating Disk, "Electrochimica Acta, $12,417-427$ (1967).

[7] R. Pollard and J. Newman, "Silicon Deposition on a Rotating Disk," J. Electrochem. Soc., 127, 744-752 (1980).

[8] A. Acrivos, "Solution of the Laminar Boundary Layer Energy Equations at High Prandtl Numbers," The Physics of Fluids, 3, 657-658 (1960).

[9] R. B. Bird, W. E. Stewart, and E. N. Lightfoot, Transport Phenomena, John Wi .ey \& Sons, Inc., New York (1960).

[10] F. Kohler and 0 . K. Rice, "Coexistence Curve of the Triethylamine-water System," J. Chem. Phys, 26, 1614-1618 (1957).

[11] W. M. Clark and R. L, Rowley, "The Mutual Diffusion Coefficient of Methanol-n-Hexane near the Consolute Point," AIChE Journal, $32,1125-1131(1986)$.

[12] J. M. H. Levelt Sengers, "Thermodynamics of Solutions near the Solvent's Critical Point," in Supercritical Fluid Technology, edited by T. J. Bruno and J. F. Ely, CRC Press, Boca Raton, FL (1991). 


\section{Appendix F}

In the vicinity of the consolute point, the diffusion coefficient may be approximated by

$$
D=D_{c}\left|\omega-\omega_{c}\right|^{3.8}
$$

and therefore,

$$
z-z_{c}-\frac{D_{c}}{4.8}\left|\omega-\omega_{c}\right|^{3.8}\left(\omega-\omega_{c}\right)
$$

The $z$ coordinate is stretched so that $\omega$ is proportional to $X$ near the consolute point:

$$
|x|^{3.8} X=z-z_{c}
$$

and equation 1 becomes

$$
v_{z} \frac{d \omega}{d X}-\frac{d}{d X}\left(\frac{D}{4.8|X|^{3.8}} \frac{d \omega}{d X}\right)
$$

The problem was reformulated with the following variables

$$
x=\frac{-X}{z_{c}^{1 / 4.8}}
$$

and

$$
W=1-\frac{\omega}{\omega_{C}}
$$


Thus equation F- 3 becomes

$$
\epsilon\left(1-|x|^{3.8} x\right)^{2} \frac{d W}{d x}-\frac{d}{d x}\left(\frac{\frac{D}{D_{c}}}{\omega_{c}^{3.8}|x|^{3.8}} \frac{d W}{d x}\right)
$$

where $\epsilon$ is defined by

$$
\epsilon-\frac{4.8 z_{c}^{3}}{\omega_{c}^{3.8} D_{c}} .
$$

This can be split into two first-order differential equations

$$
\begin{gathered}
\frac{d P}{d x}-\epsilon\left(1-|x|^{3.9_{x}}\right)^{2} \frac{\left|\omega_{c} x\right|^{3.8}}{\frac{D}{D_{c}}} P, \\
\frac{d W}{d x}=\frac{\left|\omega_{c} x\right|^{3.8}}{\frac{D}{D_{c}}} P,
\end{gathered}
$$

and solved as an initial value problem with the boundary conditions

$$
W=0, \quad P=P_{0} \text { at } x-0 .
$$

Equations F-7 and F-8 were solved using a Runge-Kutta routine. $\epsilon$ was fixed, corresponding to a given critical distance.

The concentration profiles for $\omega_{\infty}>\omega_{c}$ are shown in figure 7 . Figure 8 shows the concentration profiles when the bulk concentration is below the consolute-point composition. Qualitatively these are 


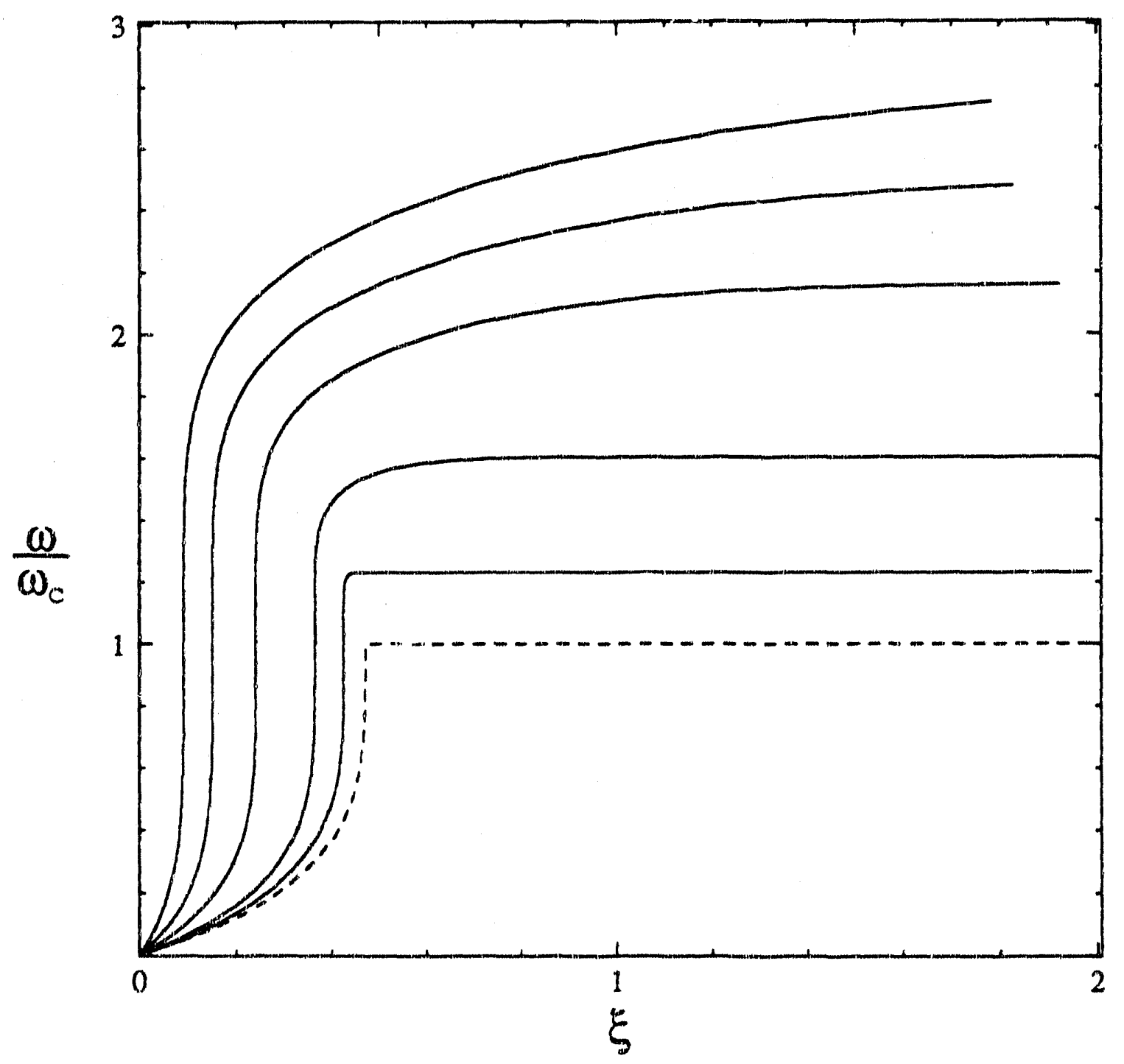

Eigure 7. Concentration profiles for bulk concentrations above or at (dashed line) the consolute-point composition. the critical exporient was 4.8 . 


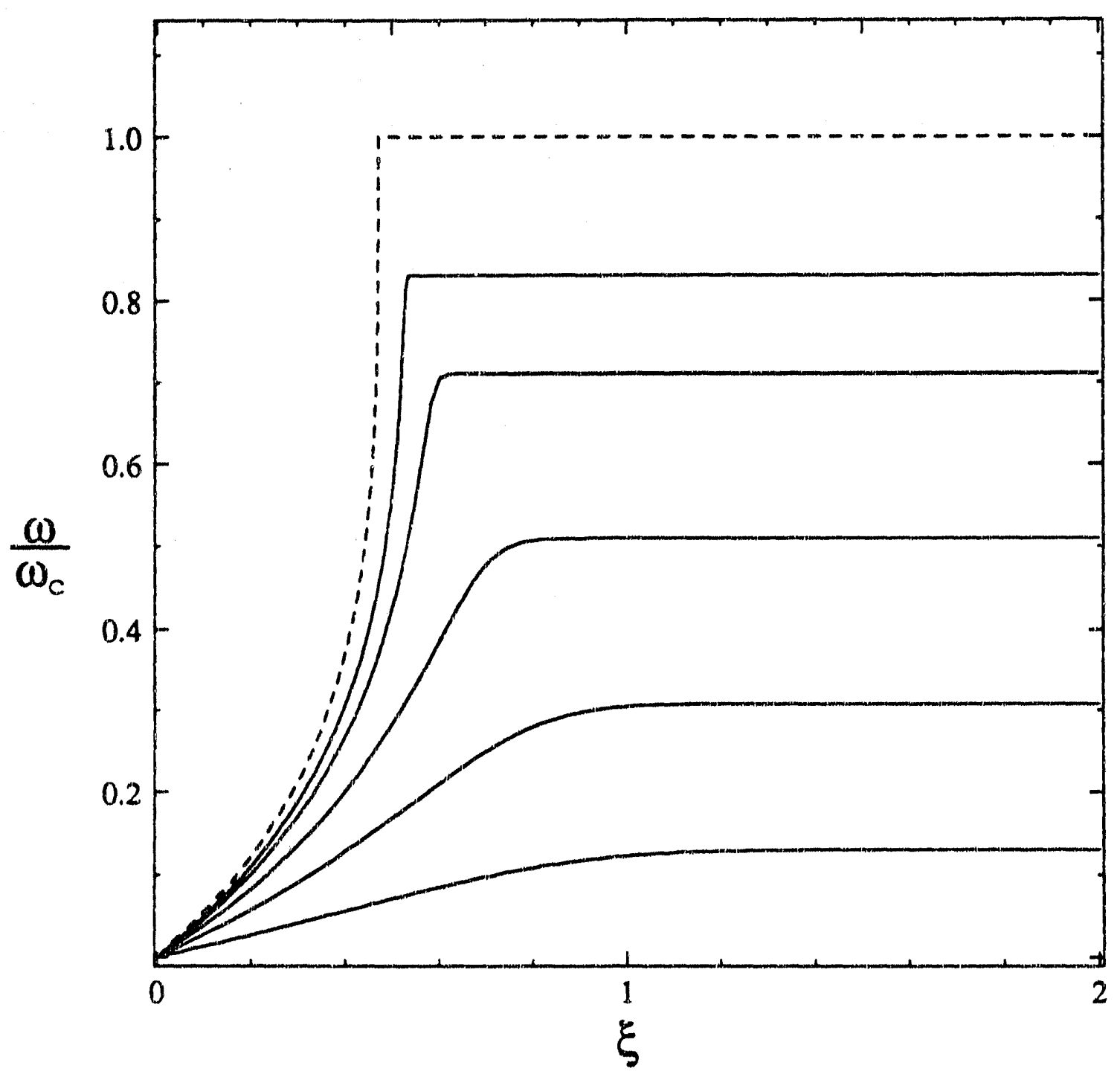

Figure 8. Concentration profiles for bulk concentrations below or at (dashed line) the consolute-point composition. The critical exponent was equal to 4.8 . 
very similar to the profiles shown in figures 2 and 3 . In both cases the concentration is plotted against $\xi$, which is now jelated to $x$ by

$$
\xi-\left(1-|x|^{3.8} x\right)\left(\frac{\epsilon}{3 \times 4.8}\right)^{1 / 3}
$$

We wished to investigate the behavior when the bulk concentration was arbitrarily close to that of the consolute composition, and to determine the maximum value of $\epsilon$.

\section{Perturbation Analysis}

Suppose there is a value of $\epsilon$ for which $d W / d x=0$ at $x=0$. The solution in the outer region would then be $W=0$ for $x<0$. For $x>0$ look for a solution of the form

$$
W=A x+B x^{2}+\ldots
$$

near $x-0$. Substituting into the differential equation and equating equal powers of $x$ gives

$$
5.8 A^{2.8} B=6 .
$$

If $\epsilon$ approaches a limit, somewhat greater that 1.5, then B approaches infinity as A goes to zero. The region where

$$
A x \approx B x^{2}
$$

defines an inner region (in the sense of a singular-perturbation 
expansion). Let

$$
\begin{aligned}
& \bar{x}-\frac{x \epsilon}{5.8 A^{3.8}} \\
& \bar{W}=\frac{\epsilon}{5.8 A^{4.8}} W
\end{aligned}
$$

The problem in the inner region becomes

$$
\left(1-\left[\frac{5.8 A^{3.8} \bar{x}}{\epsilon}\right)^{4.8}\right)^{2} 5.8 \frac{d \bar{W}}{d \bar{x}}-\frac{d}{d \bar{x}}\left[\frac{\bar{W}^{3.8}}{\bar{x}^{3.8}} \frac{d \bar{W}}{d \bar{x}}\right)
$$

with the boundary conditions

$$
\bar{W}=0 \text { and } \frac{d \bar{W}}{d \bar{x}}=1 \text { at } x=0
$$

To a zeroth approximation, $\bar{W}$ satisfies

$$
5.8 \frac{d \bar{W}}{d \bar{x}}=\frac{d}{d \bar{x}}\left(\begin{array}{ll}
\frac{\bar{W}^{3} .8}{x^{3}} & \frac{d \bar{W}}{d \bar{x}}
\end{array}\right)
$$

with the implicit solution

$$
\bar{x}^{4.8}-\int_{0}^{\vec{W}^{4.8}} \frac{d^{-4.8}}{1+5.8 \bar{W}} .
$$

As $\bar{x} \rightarrow \infty$

$$
\bar{W} \rightarrow\left(\frac{5.8 \times 3.8}{4.8}\right)^{1 / 3.8} \bar{x}^{4.8 / 3.8}
$$


or

$$
W \rightarrow\left(\frac{3.8 \epsilon}{4.8}\right)^{1 / 3.8} x^{4.8 / 3.8}
$$

The outer region satisfies equation F-6. Let $\tilde{x}-x^{3 / 2}$, and the problem becomes

$$
\frac{3.8 \epsilon}{4.8}\left(1-\tilde{x}^{3.8}\right) 2 \frac{d W}{d \tilde{x}}=\frac{d}{d \tilde{x}}\left(\frac{W^{3.8}}{\tilde{x}^{2.8}} \frac{d W}{d \tilde{x}}\right)
$$

The solution must match the inner solution as $\tilde{x} \rightarrow 0$,

$$
\mathrm{W} \rightarrow\left(\frac{3.8 \epsilon}{4.8}\right)^{1 / 3.8} \tilde{\mathrm{x}} \text { as } \tilde{\mathrm{x}} \rightarrow 0
$$

and

$$
W=1 \text { at } \bar{x}=1
$$

Thus $\epsilon$ can be adjusted, to give $W-1$ at $\tilde{x}-1$. The numerical solution gives $\epsilon=1.5069$. The concentration profiles for this condition are the dashed 1 ines in figures 7 and 8.

Mass Transfer

The rate of mass transfer to the surface of the disk is given by

$$
\frac{n}{\rho}=-\left.D \frac{d \omega}{d z}\right|_{z=0}
$$

The coordinate transformation allows us to define a dimensionless rate of mass transfer $j$ and to express it as 


$$
j=\left.\frac{1}{\omega_{c}}\left(\frac{3 D_{o}}{K}\right)^{\frac{1}{3}} \frac{d \omega}{d z}\right|_{z=0}=\left.\left(\frac{3}{4.8^{2} \epsilon}\right)^{1 / 3} \frac{d W}{d x}\right|_{X=1} .
$$

Figure 6 shows the flux to the surface of the disk from our numerical calculation as compared with the theoretical line predicted by equation 31 . The dimensionless rate of mass transfer for $\delta-4.8$ is lower than for $\delta=3$. The results are summarized below in table F-1.

Table F-1.

\begin{tabular}{|l|l|l|l|c|}
\hline$\epsilon$ & $d W /\left.d x\right|_{X-0}$ & $d W /\left.d x\right|_{X=1}$ & $\frac{\omega}{\infty}$ & $j$ \\
& & & & \\
\hline 0.01 & 0.993 & 1.00049 & 2.81242 & 2.35375 \\
0.05 & 0.965 & 1.00244 & 2.4929 & 1.37916 \\
0.1 & 0.9301 & 1.00489 & 2.34046 & 1.09731 \\
0.2 & 0.8606 & 1.00986 & 2.1591 & 0.87524 \\
0.3 & 0.7915 & 1.01488 & 2.02681 & 0.76840 \\
0.4 & 0.72285 & 1.02 & 1.91271 & 0.70166 \\
0.5 & 0.6546 & 1.02515 & 1.80678 & 0.65465 \\
0.6 & 0.58685 & 1.03041 & 1.70452 & 0.61921 \\
0.7 & 0.5196 & 1.03576 & 1.6037 & 0.59126 \\
0.8 & 0.45283 & 1.04116 & 1.50379 & 0.56846 \\
0.9 & 0.38662 & 1.04666 & 1.40617 & 0.54946 \\
1.0 & 0.32105 & 1.05233 & 1.31409 & 0.53337 \\
1.1 & 0.2561 & 1.05808 & 1.23126 & 0.51952 \\
1.2 & 0.19182 & 1.06393 & 1.1596 & 0.50746 \\
1.3 & 0.12835 & 1.06998 & 1.09871 & 0.49690 \\
1.4 & 0.06573 & 1.07615 & 1.04695 & 0.48758 \\
1.5 & 0.00419 & 1.08258 & 1.00239 & 0.47934 \\
1.5069 & 1.04752 & 0.85739 & 1.0 & 0.47881 \\
\hline
\end{tabular}




\section{Appendix $\mathrm{G}$}

C PROGRAM FOR CRITICAL DIFFUSION

IMPLICIT REAL*8 (A-H, O-Z)

DIMENSION Y $(10), a(201)$

COMMON EPS

READ *, PO, EPS , IMAX

$\mathrm{Z}=0.0$

$\mathrm{H}=+1.0 / \mathrm{DBLE}$ (IMAX)

$\mathrm{Y}(1)=\mathrm{PO}$

$Y(2)=0.0$

PRINT 102, $2, Y(1), Y(2)$

c $\quad t=(1.0-z * * 3.0) *($ eps $/ 9.0) * *(1.0 / 3.0)$

c print*, $t, 1-y(2)$

JPRINT $=10$

IMAX - IMAX *5

DO 10 I-1, IMAX

CALI RUNGE $(2, \mathrm{H}, \mathrm{Y}, \mathrm{Z})$

$a(i)-y(2)$

IF (I.EQ.JPRINT) "THEN

PRINT 101, Z,Y(1), Y(2)

c $\quad t=(1.0-z * * 3.0) *($ eps $/ 9.0) * *(1.0 / 3.0)$

c print*, $t, 1-y(2)$

JPRINT $=$ JPRINT +10

ENDIF

10 CONTINUE

101 FORMAT (3X, F10.3,2F12.5)

102 FORMAT (10X,' $\mathrm{X}$ ', 5X,' $\mathrm{P}^{\prime}, 4 \mathrm{X},{ }^{\prime} \mathrm{OMEGA}$ ' $3 \mathrm{X}, \mathrm{F} 10.3,2 \mathrm{~F} 12.5$ )

$s 1=(-1.5 * a(1)+2.0 * a(2)-0.5 * a(3)) / \mathrm{h}$

print*, sl

STOP

END

SUBROU'TINE RUNGE $(N, H, Y, Z)$

IMPLICIT REAL*8 (A-H,O-Z)

DIMENSION PHI (10), YSAVE(10), Y(10), F(10)

$M=1$

$\mathrm{H} 2=0.5 * \mathrm{H}$

1 CALL DERFUN $(Z, Y, F)$

$\mathrm{M}=\mathrm{M}+1$

GO TO $(1,2,3,4,5), M$

2 DO $22 I=1, N$

$\operatorname{YSAVE}(I)=Y(I)$

$\operatorname{PHI}(I)=F(I)$

$22 \mathrm{Y}(\mathrm{I})=\mathrm{Y}(\mathrm{I})+\mathrm{H} 2 * \mathrm{~F}(\mathrm{I})$

$\mathrm{Z}-\mathrm{Z}+\mathrm{H} 2$

GO TO 1

3 DO $33 \quad I=1, N$

$\mathrm{PHI}(I)=\mathrm{PHI}(I)+2.0 * F(I)$

$33 \mathrm{Y}(\mathrm{I})=\mathrm{YSAVE}(\mathrm{I})+\mathrm{H} 2 * \mathrm{~F}(\mathrm{I})$

GO TO 1

4 DO $44 \quad I=1, N$ 
$\operatorname{PHI}(\mathrm{I})=\operatorname{PHI}(I)+2.0 * \mathrm{~F}(\mathrm{I})$

$44 \mathrm{Y}(I)-\mathrm{YSAVE}(I)+\mathrm{H} * \mathrm{~F}(\mathrm{I})$

$\mathrm{Z}=\mathrm{Z}+\mathrm{H} 2$

GO TO 1

5 DO $55 \quad I=1, N$

$55 \mathrm{Y}(\mathrm{I})-\mathrm{YSAVE}(\mathrm{I})+(\mathrm{PHI}(\mathrm{I})+\mathrm{F}(\mathrm{I})) * \mathrm{H} / 6.0$

RETURN

END

SUBROUTINE DERFUN $(Z, Y, F)$

IMPLICIT REAL*8 $(\mathrm{A}-\mathrm{H}, \mathrm{O}-\mathrm{Z})$

DIMENSION $Y(10), F(10)$

COMMON EPS

IF (Z.NE.0.0) THEN

$F(2)=Y(1) *(Z / Y(2)) * * 2$

ELSE

$F(2)=Y(1) * *(1.0 / 3.0)$

ENDIF

$F(1)=\operatorname{EPS} *(1.0-Z * * 3) * * 2 * F(2)$

RETURN

END 

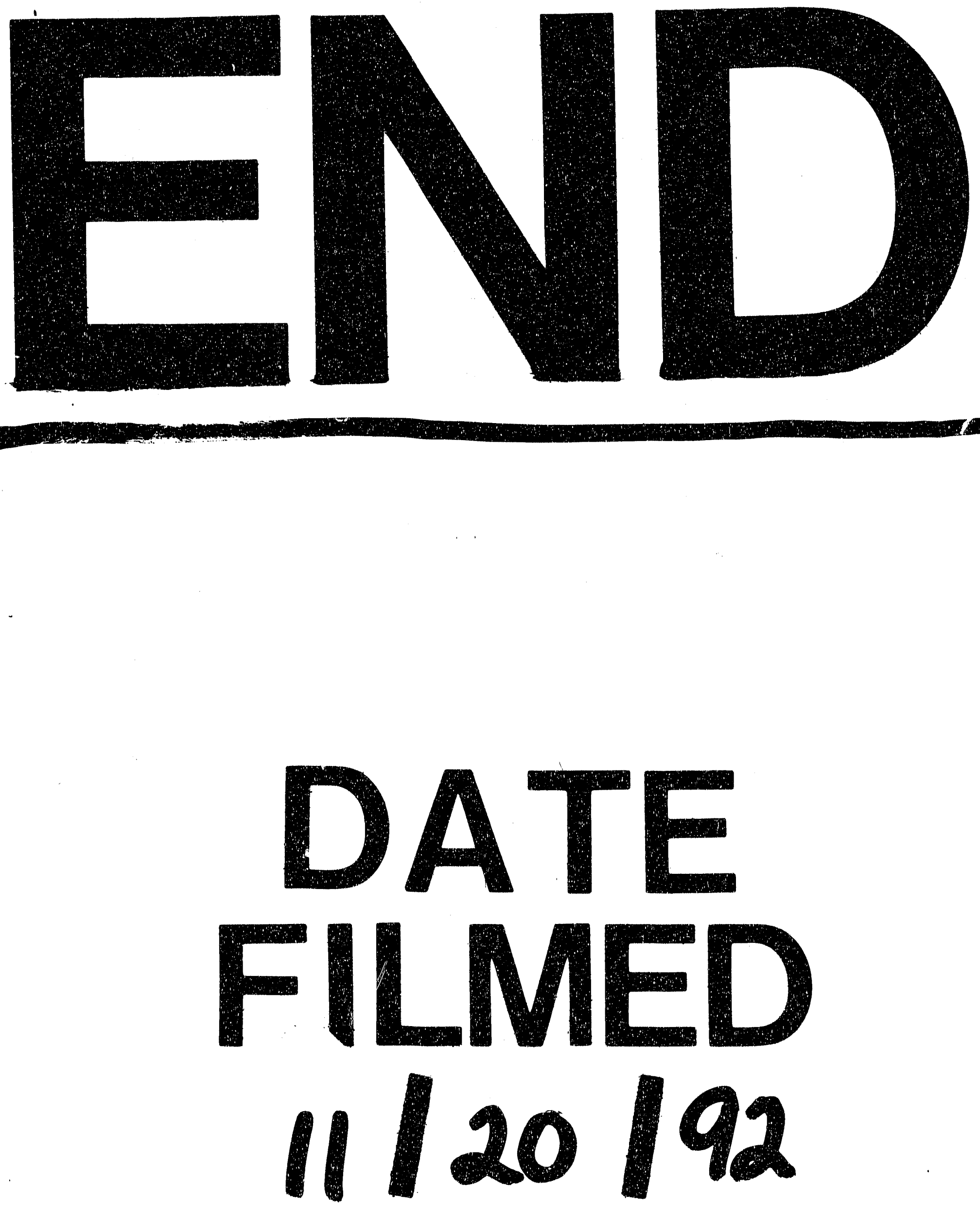
